Florida International University FIU Digital Commons

$11-18-2004$

\title{
Cross sections and Rosenbluth separations from kaon electroproduction on protons up to $\mathrm{Q}^{2}=2.35$ $(\mathrm{GeV} / \mathrm{c})^{2}$
}

Marius Coman

Florida International University

DOI: $10.25148 /$ etd.FI14060884

Follow this and additional works at: https://digitalcommons.fiu.edu/etd

Part of the Physics Commons

\section{Recommended Citation}

Coman, Marius, "Cross sections and Rosenbluth separations from kaon electroproduction on protons up to $\mathrm{Q}^{2}=2.35(\mathrm{GeV} / \mathrm{c})^{2 \text { " }}$ (2004). FIU Electronic Theses and Dissertations. 2414.

https://digitalcommons.fiu.edu/etd/2414 
Miami, Florida

CROSS SECTIONS AND ROSENBLUTH SEPARATIONS

FROM KAON ELECTROPRODUCTION

ON PROTONS UP TO $Q^{2}=2.35(\mathrm{GeV} / \mathrm{c})^{2}$

A dissertation submitted in partial fulfillment of the requirements for the degree of DOCTOR OF PHILOSOPHY

in

PHYSICS

by

Marius Coman

2005 
To: Dean R. Bruce Dunlap

College of Arts and Sciences

This dissertation, written by Marius Coman, and entitled Cross Sections and Rosenbluth Separations from Kaon Electroproduction on Protons up to $Q^{2}=2.35$ $(\mathrm{GeV} / \mathrm{c})^{2}$, having been approved in respect to style and intellectual content, is referred to you for judgment.

We have read this dissertation and recommend that it be approved.

Werner U. Boeglin

George Chang

Brian A. Raue

Jöerg Reinhold

Pete E.C. Markowitz, Major Professor

Date of Defense: November 18, 2004

The dissertation of Marius Coman is approved.

Dean R. Bruce Dunlap College of Arts and Sciences

Dean Douglas Wartzok University Graduate School

Florida International University, 2005 
(C) Copyright 2005 by Marius Coman

All rights reserved. 


\section{DEDICATION}

To my brother Emil N. Coman.

La steaua

La steaua care-a răsărit E-o cale-atît de lungă, Că mii de ani i-au trebuit Luminii să ne-ajungă. Poate de mult s-a stins în drum În depărtări albastre, Iar raza ei abia acum Luci vederii noastre. Icoana stelei ce-a murit Incet pe cer se suie; Era pe când nu s-a zărit, Azi o vedem, si nu e.
To the star

So far it is athwart the blue

To where yon star appears, That for its light to reach our view Has needed thousand years.

Maybe that ages gone it shed

Its glow, then languished in the skies, Yet only now its rays have sped Their journey to our eyes.

The icon of this now dead star

Slow in the sky it rises;

She was, while we could not her see, Now we see it, when she's vanished.

Mihai Eminescu, December 1st, 1886.

Where were you one hundred years ago?

Where will you be one hundred years from now? Tell me!

Dialogs with Father Cleopa $\uparrow$. 


\section{ACKNOWLEDGMENTS}

An impressive number of people contributed to the completion of this thesis. I would like to especially thank my advisor Pete E. C. Markowitz for his endless support (well, it's ending now) and his guidance and patience.

My wife has been an incredible friend throughout the toughest part of the project (the graveyard shifts at JLab followed in the mornings by presentations of the data analysis status). I hope to be as supportive as her (by saying I hope, I already acknowledge my inability of doing the same for her).

My gratitude goes to the committee members for their useful comments throughout the writing process. The discussions with Werner Boeglin helped me in clarifying a lot of unknowns. Having five members in the committee, all with $\mathrm{Ph}$. D. in nuclear physics, was not an easy task, but it turned out to be quite beneficial. My deepest consideration go to Judy, my advisor's wife, for her friendship and moral support.

I would like to thank Misak Sargsian for his constructive comments and help, and Petr Bydžovský from the Nuclear Physics Institute, ŘR $\breve{z}$ near Prague in Czech Republic for his theoretical calculations and very insightful comments.

A lot I learned from Bogdan Wojtsekhowski, JLab scientist, whose guidance was invaluable while building the Aerogel detector. He also knew how to celebrate with us the successful end of the project. Thank you, Bogdan. I have been fortunate to work with a research group composed of reputed scientists such as Franco Garibaldi, Douglas W.Higinbotham, Mauro Iodice, John LeRose. It's been a pleasure to work with them. I do remember though the walk through test, John. 
I would like to show my gratitude to Professor Alexandru Jipa from Bucharest University and to Ioana Niculescu and Gabriel Niculescu from Jefferson Lab who were always there for me when I needed help. I do pleasantly remember also the volleyball teammates who made the staying at JLab bearable.

This study was funded by the Department of Energy and supported by the Jefferson National Accelerator Facility. 
ABSTRACT OF THE DISSERTATION

CROSS SECTIONS AND ROSENBLUTH SEPARATIONS

FROM KAON ELECTROPRODUCTION

ON PROTONS UP TO $Q^{2}=2.35(\mathrm{GeV} / \mathrm{c})^{2}$

by

Marius Coman

Florida International University, 2005

Miami, Florida

Professor Pete E. C. Markowitz, Major Professor

The kaon electroproduction reaction $\mathrm{H}\left(e, e^{\prime} K^{+}\right) \Lambda$ was studied as a function of the four momentum transfer, $Q^{2}$, for different values of the virtual photon polarization parameter. Electrons and kaons were detected in coincidence in two High Resolution Spectrometers (HRS) at Jefferson Lab. Data were taken at electron beam energies ranging from 3.4006 to $5.7544 \mathrm{GeV}$. The kaons were identified using combined time of flight information and two Aerogel Čerenkov detectors used for particle identification. For different values of $Q^{2}$ ranging from 1.90 to $2.35 \mathrm{GeV} / \mathrm{c}^{2}$ the center of mass cross sections for the $\Lambda$ hyperon were determined for 20 kinematics and the longitudinal, $\sigma_{L}$, and transverse, $\sigma_{T}$, terms were separated using the Rosenbluth separation technique.

Comparisons between available models and data have been studied. The comparison supports the t-channel dominance behavior for kaon electroproduction. All models seem to underpredict the transverse cross section. An estimate of the kaon form factor has been explored by determining the sensitivity of the separated 
cross sections to variations of the kaon EM form factor. From comparison between models and data we can conclude that interpreting the data using the Regge model is quite sensitive to a particular choice for the EM form factors. The data from the E98-108 experiment extends the range of the available kaon electroproduction cross section data to an unexplored region of $Q^{2}$ where no separations have ever been performed. 


\section{TABLE OF CONTENTS}

1 Introduction; Physics motivation 1

1.1 Theoretical framework . . . . . . . . . . . . . . . . . 3

1.2 Kaon electroproduction: Models and previous data . . . . . . . 10

1.3 Kaon electroproduction in E98-108 . . . . . . . . . . . . 16

1.3 .1 Overview of kinematical settings . . . . . . . . . . . 18

2 Instrumentation 20

2.1 Overview of Jefferson Lab; Accelerator and Hall A beam line . . . . . 20

2.2 Beam energy measurement during E98-108 . . . . . . . . . . . 21

2.3 Beam current monitor . . . . . . . . . . . . . . . . 23

2.4 Beam position monitor - BPM . . . . . . . . . . . . . 24

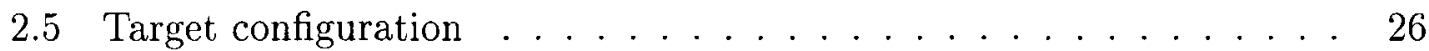

2.6 Hall A high resolution spectrometers . . . . . . . . . . . 26

2.6.1 Scintillator detectors . . . . . . . . . . . . . . . 32

2.6 .2 Vertical drift chambers ............... 33

2.6.3 Čerenkov detectors' working principle . . . . . . . . . . 35

2.6.4 Gas Čerenkov . . . . . . . . . . . . . . . . . 37

2.7 Aerogel Čerenkov detectors . . . . . . . . . . . . . . . . . . 38

2.7.1 First Aerogel Čerenkov detector, $n=1.015 \ldots \ldots 38$

2.7.2 Second Aerogel Čerenkov detector, $n=1.055 \ldots . . . .44$

2.8 Experimental data acquisition . . . . . . . . . . . . . . 43

2.8.1 Data acquisition system . . . . . . . . . . . . . . 44

2.8.2 Trigger electronics; block diagram . . . . . . . . . . . . 44

2.8.3 Electronic and computer deadtime determination . . . . . . 47

$\begin{array}{llr}3 & \text { Calibration and efficiency measurements } & 49\end{array}$

3.1 Spectrometer position and offsets determination; Optics calibration . 49

3.2 Scintillators' efficiencies . . . . . . . . . . . . . . . . . 53

3.3 Boiling corrections . . . . . . . . . . . . . . . 60

3.4 Kaon absorption . . . . . . . . . . . . . . . . 61

3.5 Vertical drift chambers efficiencies . . . . . . . . . . . . 62

3.6 Efficiency determination for the 1st aerogel Črenkov detector . . . . 71

3.7 Efficiency for the 2 nd aerogel Čerenkov detector . . . . . . . . . . . 79

3.8 Efficiency determination for the Gas Cerenkov detector . . . . . . . . 86

4 Data analysis 88

4.1 Particle identification . . . . . . . . . . . . . . . . . . 89

4.2 Background subtraction . . . . . . . . . . . . . . 97

4.3 Determination of spectrometer acceptance using the R-function . . . 102

4.4 Monte Carlo simulation . . . . . . . . . . . . . . . . . 106

4.4 .1 Radiative corrections . . . . . . . . . . . . . . . . 109 
4.5 Cross section calculation .................. . . 111 4.5.1 Systematic uncertainties . . . . . . . . . . . . 117

5 Conclusions $\quad 118$

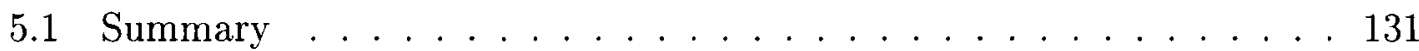

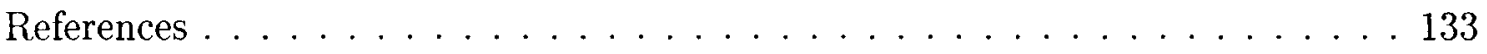

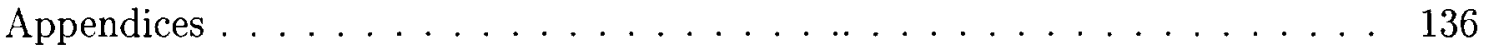

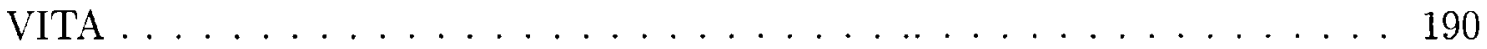




\section{LIST OF TABLES}

TABLE

PAGE

1 General characteristics of the Hall A spectrometers . . . . . . . . . . . 30

2 Table with particles' threshold momenta for different aerogel refractive indexes. . . . . . . . . . . . . . . . . . . . 42

3 Protons yield calculations for $A 1$ efficiency determination. . . . . . . . . . 74

4 Pedestal and gains for $A 1$ Čerenkov detector, $2002 \ldots \ldots$. . . . . . . . 81

$5 \quad$ Kin 1 (2001) and Kin 1 Prime (2002); calculated $Q^{2}$ and $W$ values. . . . 95

6 Unseparated cross sections and corresponding kaon yields for $\mathrm{Q}^{2}=2.35$ $\mathrm{GeV}^{2}$. Errors are statistical and include background subtraction and acceptance cuts. . . . . . . . . . . . . . . . . 113

7 Unseparated cross sections and corresponding kaon yields for $Q^{2}=1.90$ $\mathrm{GeV}^{2}$. Errors are statistical. . . . . . . . . . . . . . . 114

8 Separated cross sections. Errors are statistical and systematics added in quadrature $($ see appendix F) . . . . . . . . . . . . . . . 116

9 Systematic errors for the E981-08 experiment. . . . . . . . . . . . . . 117

10 Unseparated cross sections and corresponding correction factors: Kin 1, 23, 20, 10 and 21; The factor Rad. Correction represents the ratio of the yields as obtained from running the Monte Carlo code [ULM02] with the radiative corrections turned off and with the corrections turned on. . . . 136

11 Unseparated cross sections and corresponding correction factors: Kin 2, $24,18,3$ and 25

12 Unseparated cross sections and corresponding correction factors: Kin 4, $22,11,26$ and 19

13 Unseparated cross sections and corresponding correction factors: Kin 5, $27,17,12$ and 28

14 Unseparated cross sections and corresponding correction factors: Kin 1 Prime, 2002

15 Table with the Kinematics for Kaon Electroproduction experiment E98108 at JLAB.

20 Table with the Scintillator efficiencies; comparison all cuts and no gas Cerenkov cut. 


\section{LIST OF FIGURES}

FIGURE

PAGE

$1 \Lambda$ and $\Sigma$ Missing mass spectra obtained in the E98-108 kaon electroproduction experiment at JLab. . . . . . . . . . . . . . . . . 2

2 Tree level Feynman diagram in $H\left(e, e^{\prime} K+\right) Y$ reaction, $t$ channel exchange; kaon form factor and coupling constant indicated at vertexes. . . . . . . 5

3 Tree level Feynman diagrams in $H\left(e, e^{\prime} K+\right) Y$ reaction [PRO98]. In hadronic language the production mechanism can happen through $s, t$, or $u$ channels. . . . . . . . . . . . . . . . . . . . . 6

4 Studied Kaon electroproduction reaction, $H\left(e, e^{\prime} K+\right) Y$ in E98-108 experiment at JLab; the bold notation is used to represent vectors (p stands for $\vec{p}) \ldots \ldots \ldots \ldots \ldots \ldots$

5 Qualitative description of the kaon electroproduction. . . . . . . . . . 13

6 Photoproduction cross section (in the center-of-mass) versus $\cos \left(\theta_{K}^{C M}\right)$, $W=2.017 \mathrm{GeV}$. Data from [MCN04] . . . . . . . . . . . . . . . 14

7 Polarized LT' interference response function versus $W$ from electroproduction data; $W=1.625-1.975 \mathrm{GeV}, Q^{2}=0.7 \mathrm{GeV}^{2}$; figure from [NAS04].

8 Kaon electroproduction: previous world data [NIC98]; Cross sections are extrapolated [BEB77] to $\mathrm{W}=2.15 \mathrm{GeV} \ldots \ldots \ldots \ldots$

9 The accelerator at Jefferson Lab.[HWEBA] . . . . . . . . . . . . . . . 21

10 Ratio of the charge measurements as extracted from the EPICS datastream for the E98-108 experiment. . . . . . . . . . . . . . . . 24

11 Rastered beam size on target for E98-108, 2001. . . . . . . . . . . 25

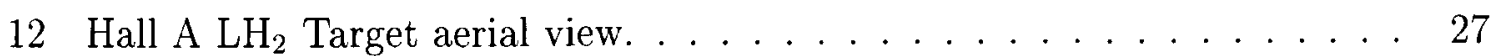

13 Hall $\mathrm{A} \mathrm{LH}_{2}$ Target-side view. . . . . . . . . . . . . . . . . 28

14 Layout of superconducting magnets in Hall A at Jefferson Lab. . . . . . . 29

15 Layout of high resolution spectrometers in Hall A at Jefferson Lab. . . . . 29

16 Schematic layout of the scintillator detectors. . . . . . . . . . . . 32

17 Particle velocities relative to the speed of light as a function of particles' momentum; Threshold velocity for aerogel index $n=1.055 \pm 0.002$ and $n=1.015 \pm 0.002$ are indicated. 
18 Gas Cerenkov ADC sum distribution fitted to a Gaussian. . . . . . . . . . 38

19 First diffusion box Aerogel Čerenkov detector. . . . . . . . . . . . . . . . 40

20 Second diffusion box-Aerogel Čerenkov detector. . . . . . . . . . . . . . . 42

21 Single arm trigger for E98-108; modified figure taken from [MIC02]. . . . 45

22 Coincidence trigger for E98-108; modified figure taken from [MIC02]. . . . 46

23 Electronic deadtime vs run number, 1 st set of runs. . . . . . . . . . . . 48

24 Electronic deadtime vs run number, 2nd set of runs. . . . . . . . . . 48

25 Sieve slit ${ }^{12} \mathrm{C}$ target, Electron Arm: Non-dispersive position at the collimator (in $m$ ) versus dispersive position at the collimator (in $m$ ) . . . 50

26 Sieve slit ${ }^{12} \mathrm{C}$ target, Hadron Arm: Non-dispersive position at the collimator (in $m$ ) versus dispersive position at the collimator (in $m$ ) . . . 50

$27 z$ coordinate of the reaction point in the target (in meters) vs rastered beam position ( $\mathrm{x}$ coordinate at the Hall A center in meters), E arm. . . . 51

$28 z$ coordinate of the reaction point in the target (in meters) vs rastered beam position ( $\mathrm{x}$ coordinate at the Hall $\mathrm{A}$ center in meters), $\mathrm{H}$ arm. . . . 51

29 Beta optimization, Left Arm for electrons. . . . . . . . . . . . . 52

30 Total Scintillator efficiencies. . . . . . . . . . . . . . . . . 54

31 Scintillator efficiencies in the Electron arm, $S 1$ plane. . . . . . . . . . 56

32 Scintillator efficiencies in the Electron arm, $S 2$ plane. . . . . . . . . 57

33 Scintillator efficiencies in the Hadron arm, $S 1$ plane. . . . . . . . . . 58

34 Scintillator efficiencies in the Hadron arm, $S 2$ plane. . . . . . . . . . 59

35 Scintillator efficiencies in the electron arm ( $S 1$ plane) for different types of particles - protons and pions. . . . . . . . . . . . 60

36 Scintillator efficiencies in the hadron arm ( $S 2$ plane) for different types of particles - protons and pions. ............. 61

37 Distribution of the number of hits in $V D C_{\text {Electron }} \ldots \ldots \ldots \ldots$

38 Distribution of the number of hits in $V D C_{\text {Hadron }} \ldots \ldots \ldots \ldots$

39 VDCs' Tracking efficiency for E98-108. . . . . . . . . . . . . 67

40 VDCs' Firing efficiency for E98-108. . . . . . . . . . . 68

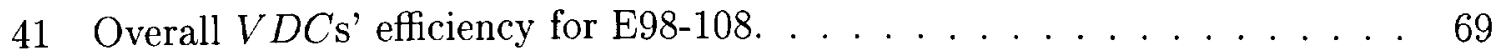


42 A1 efficiency for protons (knock-on electrons), first degree polynomial fit together with $1 \sigma, 2 \sigma$ and $3 \sigma$ bands. . . . . . . . . . 75

43 A1 efficiency for protons as a function of hadrons' momenta. . . . . . . 76

44 A1 distribution for electrons, 2001; The position of the single photoelectron peak is shown. . . . . . . . . . . . . . . 77

45 A1 distribution for electrons, 2001, Mean number of PE from Poisson fit

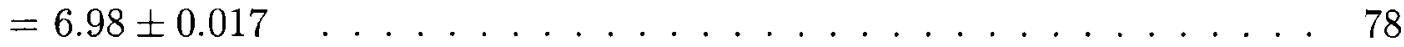

46 A1 distribution for $\pi, 2001$, Mean number of PE from Poisson fit $=6.28 \pm$

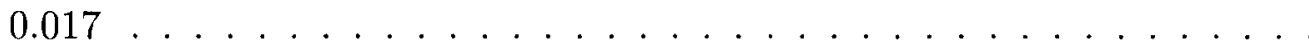

47 A1 distribution for $\pi, 2002$, Mean number of PE from Poisson fit $=4.32 \pm$

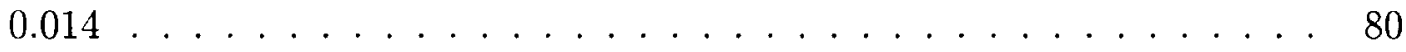

$48 \quad A 2$ Čerenkov detector: distribution of number of $P E$ for $\pi$, Kin 1, 2001. . 82

$49 A 2$ Cerenkov detector: distribution of number of $P E$ for $\pi$, Kin 1 prime, 2002

$50 A 2$ correction factor for a 1 to $3 P E$ cut as a function of particles' momenta; a 2 to $4 P E$ cut was used for all kinematics. . . . . . . . . . . 84

$51 A 2$ correction factor for a 4 to $8 P E$ cut as a function of particles' momenta. 84

$52 \quad A 2$ Cerenkov detector: distribution of number of $P E$ for kaons, Kin 4. . $\quad 85$

$53 A 2$ Čerenkov detector: distribution of number of $P E$ for kaons, Kin 5. . . 85

54 Gas Čerenkov efficiency for electrons as a function of particles' momenta . 87

55 Corrected coincidence time spectra, identifying peaks after $A 1$ and $A 2$ cuts; Aerogel cuts are not optimized; $x$ axis is in ns. . . . . . . . . . . . 90

56 Fitted Corrected coincidence time spectra; proton, $K$ and $\pi$ peaks after $A 1$ and $A 2$ cuts; $\mathrm{x}$ axis is in $\mathrm{ns}$.

57 Coincidence time spectra, proton, $K, \pi$ and accidental regions; $\pi$ peak is scaled by $1.5 ; x$ axis is in ns.

58 Yields from corrected coincidence time spectra. . . . . . . . . . . . . . 93

59 Corrected coincidence time spectra, before applying the aerogel cuts ( $x$ axis is in ns). Pions and kaons cannot be distiguished. . . . . . . . .

60 Corrected coincidence time spectra, after applying the aerogel cuts ( $x$ axis is in $n s)$.

61 Number of $P E \times 100$ produced in $A 1$ by $\pi$ that also fired $A 2$. 
62 Missing Mass spectra, no cuts applied. . . . . . . . . . . . . . . . . . 97

63 Missing mass spectra, with only coincidence time cut applied. . . . . . . . 98

64 Missing mass spectra, with TC and aerogels cuts applied, Kin 20 ( $x$ axis is in $\left.\mathrm{MeV} / \mathrm{c}^{2}\right) \ldots \ldots \ldots \ldots$. . . . . . . . . . . . . . . . 99

65 Missing mass spectra, with TC and aerogels cuts applied ( $x$ axis is in $\left.\mathrm{MeV} / \mathrm{c}^{2}\right)$, Kin 23. . . . . . . . . . . . . . . . . 100

66 Missing mass spectra, accidentals fitting $\left(x\right.$ axis is in $\left.\mathrm{MeV} / \mathrm{c}^{2}\right) \ldots \ldots 101$

67 Missing mass spectra with accidental and $\Lambda$ fits $\left(x\right.$ axis is in $\left.\mathrm{MeV} / \mathrm{c}^{2}\right) \ldots 102$

68 Fitted missing mass spectra with accidentals subtracted, for Kin 3 ( $x$ axis is in $\left.\mathrm{MeV} / \mathrm{c}^{2}\right) \ldots \ldots \ldots \ldots . \ldots \ldots$

69 Missing mass spectra with accidental subtraction for Kin 20. . . . . . . 104

70 Missing mass spectra with accidental subtraction for Kin 1. . . . . . . 105

71 Missing mass spectra with accidental subtraction for Kin 24 . . . . . . 106

72 Missing mass spectra with no R-function cut, Kin 1 ( $x$ axis is in $\mathrm{MeV} / \mathrm{c}^{2}$ ). 107

73 Missing Mass spectra, with R-function cut applied, Kin 1 ( $x$ axis is in $\left.\mathrm{MeV} / \mathrm{c}^{2}\right) \ldots \ldots \ldots \ldots \ldots \ldots$

$74 \Lambda$ missing mass Monte Carlo simulation and data $\left(x\right.$ axis is in $\left.\mathrm{MeV} / \mathrm{c}^{2}\right) . \quad 111$

75 Kaon electroproduction: E98-108 data shown; $\Lambda$ cross sections (Kin 10 at $Q^{2}=1.90$ and at $W=2.14$, and Kin 1 at $Q^{2}=2.35$ and $\left.W=1.80\right)$ are extrapolated [BEB77] to $\mathrm{W}=2.15 \mathrm{GeV}$.

76 Longitudinal cross section, $\sigma_{L}^{\Lambda}$ versus $Q^{2}$ for $W=1.84$, comparison E98108 data and available models. Theoretical calculations from [BYD04], solid line is K-Maid [MAR03], dotted bold line is SLA [DAV96], [MIZ98] double dotted line is M2(WAA) [WIL92], dotted-interrupted line is $\mathrm{M} 2(\mathrm{WWW})$ [ADE88], dotted line is Regge [GUI00], the square symbol is data from [NIC98], the triangle is data from [MOH03] and the star is E98-108 data.

77 Transverse cross section, $\sigma_{T}^{\Lambda}$ versus $Q^{2}$ for $W=1.84$, comparison E98-108 data and available models. Theoretical calculations from [BYD04] . . . 122

78 Longitudinal cross section, $\sigma_{L}^{\Lambda}$ versus $W$ for $Q^{2}=1.90 \mathrm{Gev}^{2}$, comparison E98-108 data and available models. Theoretical calculations from [BYD04].

79 Transverse cross section, $\sigma_{T}^{\Lambda}$ versus $W$ for $Q^{2}=1.90$, comparison E98-108 data and available models. Theoretical calculations from [BYD04]. 
80 Longitudinal cross section, $\sigma_{L}^{\Lambda}$ versus $W$ for $Q^{2}=2.35$, comparison data and available models. Theoretical calculations from [BYD04]. . . . . 125

81 Transverse cross section, $\sigma_{T}^{\Lambda}$ versus $W$ for $Q^{2}=2.35$, comparison E98-108 data and available models. Theoretical calculations from [BYD04].

82 Predictions of the Regge model for the $\sigma_{L}, Q^{2}=1.90 \mathrm{GeV}^{2}$. The EM form factor for the kaon trajectory is scaled by the factor emf $f_{k}$. Theoretical calculations from [BYD04], solid line is Regge [GUI00], the square symbol is data from [MOH03], the triangle is data from [NIC98] and star is E98108 data.

83 Predictions of the Regge model for the $\sigma_{T}, Q^{2}=1.90 \mathrm{GeV}^{2}$. The EM form factor for the kaon trajectory is scaled by the factor $e m f f_{k}$. Theoretical calculations from [BYD04].

84 Predictions of the Regge model for the $\sigma_{L}, Q^{2}=2.35 \mathrm{GeV}^{2}$. The EM form factor for the kaon trajectory is scaled by the factor emf $f_{k}$. Theoretical calculations from [BYD04].

85 Predictions of the Regge model for the $\sigma_{T}, Q^{2}=2.35 \mathrm{GeV}^{2}$. The EM form factor for the kaon trajectory is scaled by the factor $e m f f_{k}$. Theoretical calculations from [BYD04].

86 Ratio of the longitudinal to transverse cross section $\left(\sigma_{L} / \sigma_{T}\right)^{\Lambda}$ versus $Q^{2}, W=1.84 \mathrm{GeV}$, comparison of E98-108 data and available models. Theoretical calculations from [BYD04].

$87 \Lambda$ and $\Sigma$ Missing mass $\left(x\right.$ axis is in $\left.\mathrm{MeV} / \mathrm{c}^{2}\right)$, Kin. 23. . . . . . . 142

$88 \Lambda$ and $\Sigma$ Missing mass $\left(x\right.$ axis is in $\left.\mathrm{MeV} / \mathrm{c}^{2}\right)$, Kin. 2. . . . . . . . . 142

$89 \Lambda$ and $\Sigma$ Missing mass $\left(x\right.$ axis is in $\left.\mathrm{MeV} / \mathrm{c}^{2}\right)$, Kin. 24. . . . . . 143

$90 \Lambda$ and $\Sigma$ Missing mass $\left(x\right.$ axis is in $\left.\mathrm{MeV} / \mathrm{c}^{2}\right)$, Kin. 18. . . . . . 143

$91 \Lambda$ and $\Sigma$ Missing mass $\left(x\right.$ axis is in $\left.\mathrm{MeV} / \mathrm{c}^{2}\right)$, Kin. $4 \ldots \ldots . \ldots 144$

$92 \Lambda$ and $\Sigma$ Missing mass $\left(x\right.$ axis is in $\mathrm{MeV} / \mathrm{c}^{2}$ ), Kin. 22. . . . . . . 144

$93 \Lambda$ and $\Sigma$ Missing mass $\left(x\right.$ axis is in $\left.\mathrm{MeV} / \mathrm{c}^{2}\right)$, Kin. 3. . . . . . 145

$94 \Lambda$ and $\Sigma$ Missing mass $\left(x\right.$ axis is in MeV/c $\mathrm{c}^{2}$ ), Kin. 25. . . . . . . 145

$95 \Lambda$ and $\Sigma$ Missing mass $\left(x\right.$ axis is in $\left.\mathrm{MeV} / \mathrm{c}^{2}\right)$, Kin. $19 \mathrm{~A} \ldots \ldots . . . .146$

$96 \Lambda$ and $\Sigma$ Missing mass $\left(x\right.$ axis is in $\left.\mathrm{MeV} / \mathrm{c}^{2}\right)$, Kin. 26. . . . . . . 146

$97 \Lambda$ and $\Sigma$ Missing mass $\left(x\right.$ axis is in $\left.\mathrm{MeV} / \mathrm{c}^{2}\right)$, Kin. 11. . . . . . 147

$98 \Lambda$ and $\Sigma$ Missing mass $\left(x\right.$ axis is in $\left.\mathrm{MeV} / \mathrm{c}^{2}\right)$, Kin. 10. . . . . . . 147 
$99 \Lambda$ and $\Sigma$ Missing mass $\left(x\right.$ axis is in $\left.\mathrm{MeV} / \mathrm{c}^{2}\right)$, Kin. $5 \ldots \ldots . \ldots 148$

$100 \Lambda$ and $\Sigma$ Missing mass $\left(x\right.$ axis is in $\mathrm{MeV} / \mathrm{c}^{2}$ ), Kin. 27. . . . . . 148

$101 \Lambda$ and $\Sigma$ Missing mass $\left(x\right.$ axis is in $\left.\mathrm{MeV} / \mathrm{c}^{2}\right)$, Kin. 17 A. . . . . . . . 149

$102 \Lambda$ and $\Sigma$ Missing mass $\left(x\right.$ axis is in $\mathrm{MeV} / \mathrm{c}^{2}$ ), Kin. 12. . . . . . . 149

$103 \Lambda$ and $\Sigma$ Missing mass $\left(x\right.$ axis is in $\mathrm{MeV} / \mathrm{c}^{2}$ ), Kin. $19 \mathrm{~B} \ldots \ldots$

$104 \Lambda$ and $\Sigma$ Missing mass $\left(x\right.$ axis is in $\left.\mathrm{MeV} / \mathrm{c}^{2}\right)$, Kin. $21 \ldots . \ldots 150$

$105 \Lambda$ and $\Sigma$ Missing mass $\left(x\right.$ axis is in $\mathrm{MeV} / \mathrm{c}^{2}$ ), Kin. $28 \ldots \ldots 151$

$106 \Lambda$ and $\Sigma$ Missing mass $\left(x\right.$ axis is in $\mathrm{MeV} / \mathrm{c}^{2}$ ), Kin. $17 \mathrm{~B} \ldots \ldots . . . .151$

$107 \Lambda$ and $\Sigma$ Missing mass $\left(x\right.$ axis is in $\mathrm{MeV} / \mathrm{c}^{2}$ ), Kin. 1 Prime, $2002 \ldots . .152$

$108 \Lambda$ and $\Sigma$ Missing mass $\left(x\right.$ axis is in $\mathrm{MeV} / \mathrm{c}^{2}$ ), Kin. 2, without the Rfunction cut. . . . . . . . . . . . . . . 153

$109 \Lambda$ and $\Sigma$ Missing mass ( $x$ axis is in $\mathrm{MeV} / \mathrm{c}^{2}$ ), Kin. 23, without the Rfunction cut. . . . . . . . . . . . . . . 153

$110 \Lambda$ and $\Sigma$ Missing mass ( $x$ axis is in $\mathrm{MeV} / \mathrm{c}^{2}$ ), Kin. 24, without the Rfunction cut.

$111 \Lambda$ and $\Sigma$ Missing mass $\left(x\right.$ axis is in $M e V / c^{2}$ ), Kin. 1 , without the $R$ function cut.

$112 \Lambda$ channel, $\phi$ and $t$ coverage, Kin 1.

$113 \Lambda$ channel, $\phi$ and $t$ coverage, Kin 23.

$114 \Lambda$ channel, $\phi$ and $t$ coverage, Kin 20 .

$115 \Lambda$ channel, $\phi$ and $t$ coverage, Kin 2. 156

$116 \Lambda$ channel, $\phi$ and $t$ coverage, Kin 24 .

$117 \Lambda$ channel, $\phi$ and $t$ coverage, Kin 18 .

$118 \Lambda$ channel, $\phi$ and $t$ coverage, Kin 3 .

$119 \Lambda$ channel, $\phi$ and $t$ coverage, Kin 25.

$120 \Lambda$ channel, $\phi$ and $t$ coverage, Kin 4 .

$121 \Lambda$ channel, $\phi$ and $t$ coverage, Kin 22 .

$122 \Lambda$ channel, $\phi$ and $t$ coverage, Kin 19 . 160

$123 \Lambda$ channel, $\phi$ and $t$ coverage, Kin 26 . 
$124 \Lambda$ channel, $\phi$ and $t$ coverage, Kin 11 . 161

$125 \Lambda$ channel, $\phi$ and $t$ coverage, Kin $10 . \ldots \ldots \ldots 16 \ldots \ldots \ldots$

$126 \Lambda$ channel, $\phi$ and $t$ coverage, Kin $11 \ldots \ldots \ldots \ldots$

$127 \Lambda$ channel, $\phi$ and $t$ coverage, Kin $10 . \ldots \ldots \ldots 16 \ldots \ldots \ldots$

128 Rosenbluth separation, $W=1.905 \mathrm{GeV}, Q^{2}=1.90(\mathrm{GeV} / \mathrm{c})^{2} \ldots \ldots \ldots$

129 Rosenbluth separation, $W=2.14 \mathrm{GeV}, Q^{2}=1.90(\mathrm{GeV} / \mathrm{c})^{2} \ldots \ldots \ldots$

130 Rosenbluth separation, $W=2 . \mathrm{GeV}, Q^{2}=1.90(\mathrm{GeV} / \mathrm{c})^{2} \ldots \ldots \ldots \ldots$

131 Rosenbluth separation, $W=1.8 \mathrm{GeV}, Q^{2}=2.35(\mathrm{GeV} / \mathrm{c})^{2} \ldots \ldots \ldots$

132 Rosenbluth separation, $W=1.85 \mathrm{GeV}, Q^{2}=2.35(\mathrm{GeV} / \mathrm{c})^{2} \ldots \ldots \ldots$

133 Rosenbluth separation, $W=1.98 \mathrm{GeV}, Q^{2}=2.35(\mathrm{GeV} / \mathrm{c})^{2} \ldots \ldots \ldots$

134 Rosenbluth separation, $W=2.08 \mathrm{GeV}, Q^{2}=2.35(\mathrm{GeV} / \mathrm{c})^{2} \ldots \ldots \ldots$

135 Rosenbluth separation, $W=1.94 \mathrm{GeV}, Q^{2}=1.90(\mathrm{GeV} / \mathrm{c})^{2} \ldots \ldots \ldots$

136 Rosenbluth separation, $W=1.80 \mathrm{GeV}, Q^{2}=2.35(\mathrm{GeV} / \mathrm{c})^{2}$. Kin 1 prime included . . . . . . . . . . . . . . . . 166

$137 W$ versus $Q^{2}$ phase space (for both $\Lambda$ and $\Sigma$ channels) for Kin $1 . \quad \ldots . .169$

$138 W$ versus $Q^{2}$ phase space (for both $\Lambda$ and $\Sigma$ channels) for Kin 23 . . . . 169

$139 W$ versus $Q^{2}$ phase space (for both $\Lambda$ and $\Sigma$ channels) for Kin $20 \ldots \ldots$

$140 W$ versus $Q^{2}$ phase space (for both $\Lambda$ and $\Sigma$ channels) for Kin $2 . \quad \ldots 170$

$141 W$ versus $Q^{2}$ phase space (for both $\Lambda$ and $\Sigma$ channels) for Kin 24 . . . 171

$142 W$ versus $Q^{2}$ phase space (for both $\Lambda$ and $\Sigma$ channels) for Kin 18 . . . 171

$143 W$ versus $Q^{2}$ phase space (for both $\Lambda$ and $\Sigma$ channels) for Kin $3 . \quad \ldots$.

$144 W$ versus $Q^{2}$ phase space (for both $\Lambda$ and $\Sigma$ channels) for Kin 25 . . . 172

$145 W$ versus $Q^{2}$ phase space (for both $\Lambda$ and $\Sigma$ channels) for Kin $4 . \quad \ldots$.

$146 W$ versus $Q^{2}$ phase space (for both $\Lambda$ and $\Sigma$ channels) for Kin 22 . . . 173

$147 W$ versus $Q^{2}$ phase space (for both $\Lambda$ and $\Sigma$ channels) for Kin 19 . . . 174

$148 W$ versus $Q^{2}$ phase space (for both $\Lambda$ and $\Sigma$ channels) for Kin 26 . . . . 174

$149 W$ versus $Q^{2}$ phase space (for both $\Lambda$ and $\Sigma$ channels) for Kin 11 . . . 175

$150 W$ versus $Q^{2}$ phase space (for both $\Lambda$ and $\Sigma$ channels) for Kin 10 . . . 175 
$151 W$ versus $Q^{2}$ phase space (for both $\Lambda$ and $\Sigma$ channels) for Kin $10 \ldots \ldots$

$152 W$ versus $Q^{2}$ phase space (for both $\Lambda$ and $\Sigma$ channels) for Kin $12 \ldots 176$

$153 W$ versus $Q^{2}$ phase space (for both $\Lambda$ and $\Sigma$ channels) for Kin $28 . \ldots 177$ 


\section{Introduction; Physics motivation}

Understanding the structure of nuclei, the forces between nucleons and their interaction in terms of nucleonic degrees of freedom (quarks and gluons) is the longterm objective and focus of both experimental and theoretical nuclear physics. Of high interest are the experiments in the energy range available at Jefferson Lab, such as the E98-108 experiment described in this thesis, where the interface between a hadronic description of nuclei and a description in terms of quarks and gluons is explored. Hadronic field theories provide an effective description of the dynamics of the electroproduction process in the few $\mathrm{GeV}$ (low and intermediate) energy range. Even though these theories do not explicitly contain the quark degrees of freedom they account for the basic features which follow from the Quantum Chromodynamics (QCD). Therefore the experimental verification of effective hadronic theories may play a crucial role in understanding the QCD dynamics of hadrons.

The electroproduction experiments provides access to a wider range of observables due to the fact that the virtual photon can exhibit longitudinal polarization (the only polarization present in photoproduction is the transverse polarization), and its polarization together with its mass $\left(-Q^{2}\right)$ can be varied allowing a variety of constraints on the parameters (strong coupling constants, transition magnetic moments). The kaon electroproduction experiment, E98-108, performed at Thomas Jefferson National Accelerator facility (JLab) involves the determination of the exclusive kaon production cross sections in the ${ }^{1} \mathrm{H}\left(e, e^{\prime} K^{+}\right) Y$ reaction, with $Y$ being 


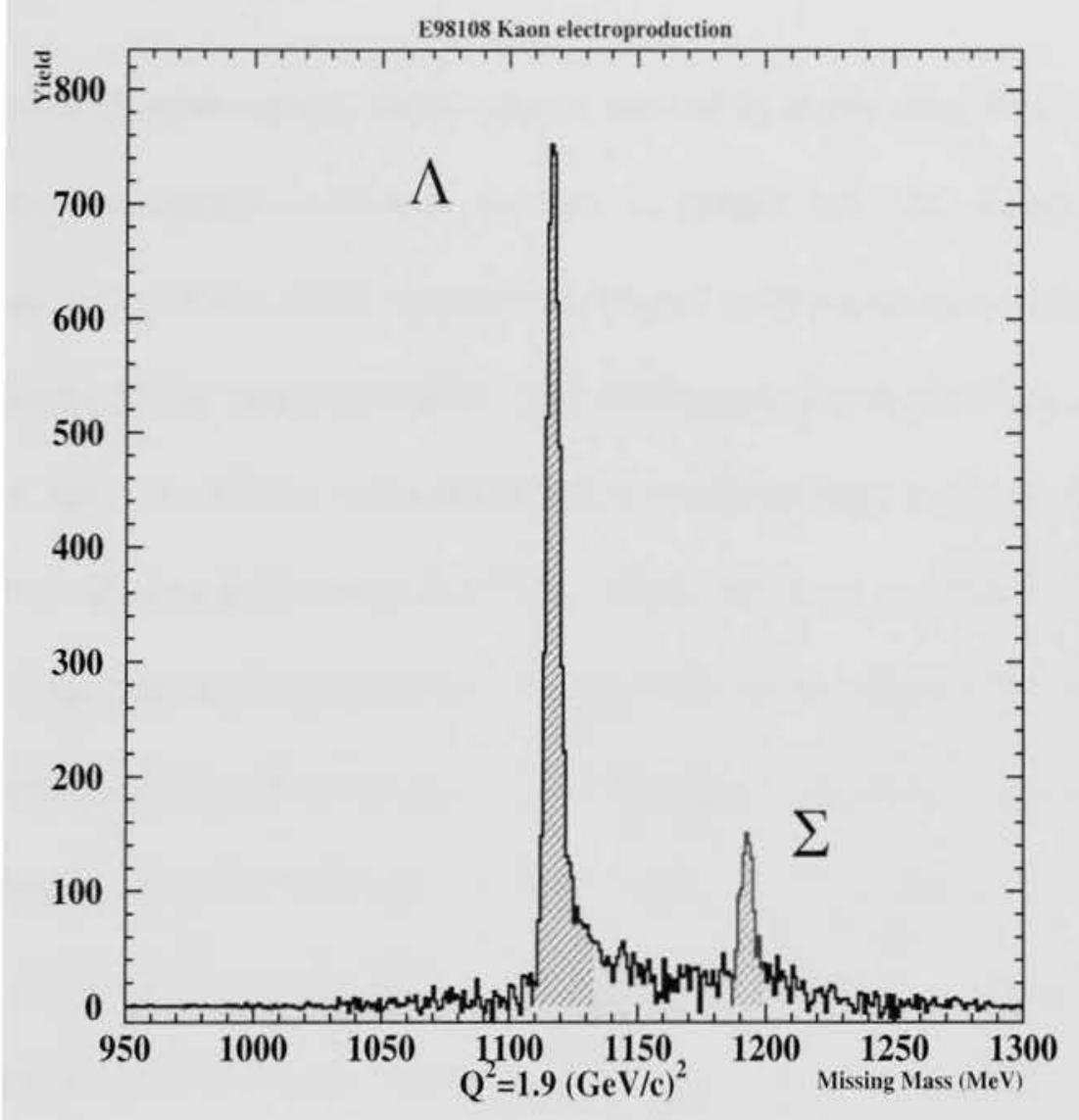

Figure 1: $\Lambda$ and $\Sigma$ Missing mass spectra obtained in the E98-108 kaon electroproduction experiment at JLab.

the $\Lambda$ or $\Sigma$ hyperons (see Fig. 1). The study was done in Hall A at Jefferson Lab in the framework of the experimental program to measure kaons, a member of the pseudoscalar (strange) meson multiplet. 


\subsection{Theoretical framework}

The structure of nucleons and nuclei can be probed by scattering electrons off target nuclei. Electromagnetic scattering can give an insight into the charge and current distribution of scatterers, which are directly related to the dynamics of the interaction of constituents of the target particles. The advantage of using electron scattering is in the fact that one photon approximation is overhelmingly a good approximation and can be calculated precisely [FEY72]. This allows us to separate the hadron dynamics from the electroproduction cross section with a high degree of reliability. It is generally accepted that starting at four momentum transfers of $Q^{2} \geq 1 \mathrm{GeV}^{2}$ the virtual photon probes the sub-nucleonic structure of the hadron (see e.g.[CLO79]). Electron beams in the energy range used at Thomas Jefferson National Accelerator Facility (JLab) are therefore high enough to give an access to the sub-nucleonic structure of hadrons. However these energies probe only nonperturbative aspects of QCD. Since QCD in the nonperturbative region is not a solved theory, the role of the effective hadronic models are very important in understanding the underlying physics of hadrons electroporduction.

One of the important features of the restricted energy and momentum transfers is the low multiplicity of the produced particles at the final state. This situation can be exploited for exclusive electroproduction of strangeness since in this case relatively few particles need to be be detected in order to provide the complete exclusiveness of the reaction. 
We start with a discussion of the general framework of exclusive electroproduction of $K$ and $\Lambda$ pairs from the proton target. In this case the five-fold differential electroproduction cross section (for exclusive kaon production) can be written [WIL92] in terms of the transition matrix amplitude and kinematical factors as:

$$
\begin{array}{r}
\frac{d^{5} \sigma}{d E^{\prime} d \Omega_{e^{\prime}} d \Omega_{K}}=\frac{(2 \pi)^{-5} M_{p} M_{Y} M_{e}^{2}|\vec{k}|^{2} E^{\prime}}{2\left[\left(e_{\mu} \cdot p_{\mu}\right)^{2}-M_{e}^{2} M_{p}^{2}\right]^{1 / 2} E_{K} E_{Y}} \\
\frac{|\vec{k}| E_{Y} E_{K}}{\vec{k} \cdot\left(E_{Y} \vec{k}-E_{K} \vec{y}\right)}<\left|t_{f i}\right|^{2}>
\end{array}
$$

where the cross section is differential in the energy of the scattered electron, $E^{\prime}$, the solid angle of the scattered electron, $d \Omega_{e^{\prime}}$ and the solid angle of the detected kaon, $d \Omega_{K}$. The quantities involved in the cross section formula (1) are: $e_{\mu}$, (the four momenta of the electron), $p_{\mu}$, (the four momenta of the target proton), $M_{e}, M_{p}, M_{Y}$ (the masses of the electron, proton and undetected hyperon, $\Lambda$ or $\Sigma$ respectively), $E_{K}$ and $E_{Y}$ (the energies of the kaon and the hyperon respectively), $\vec{y}, \vec{k}$, (the three momenta of the hyperon and the kaon respectively) and $\left\langle\left|t_{f i}\right|^{2}>\right.$ (the square of the transition matrix element). The transition amplitude $<\left|t_{f i}\right|>$ (in the one photon exchange approximation) is expressed [WIL93] in terms of the leptonic and hadronic currents mediated by the photon propagator:

$$
t_{f i}=\frac{J^{L} \cdot J^{H}}{q^{2}}
$$

where $J_{L}$ and $J_{H}$ are the leptonic and hadronic currents respectively, and $q$ is the difference between the four momenta of the incident electron and the four momenta of the scattered electron, $q=e_{\mu}-e_{\mu}^{\prime}$. The hadronic current (containing the information 
about the structure of the target nucleon) can be represented as a linear combination of six elementary transition amplitudes [WIL92] (Lorentz invariant terms that are functions of the Mandelstam variables, $\left.A_{i}\left(q^{2}, s, t, u\right)\right)$ :

$$
J_{\mu}^{H}=\bar{u}_{Y}\left[\sum_{i=1}^{6} A_{i} M_{\mu}^{i}\right] u_{p}
$$

where $M_{\mu}^{i}$ are covariant bilinear matrices (this ensures that the whole set of coupling constants may be multiplied by an arbitrary phase space - in the tree level approximation - [BYC03] and the main coupling constant $g_{p K \Lambda}$ has the same sign for different models), $u_{p}$ is the standard Dirac spinor for the proton and $\bar{u}_{Y}$ is the complex conjugate of the hyperons' spinor.

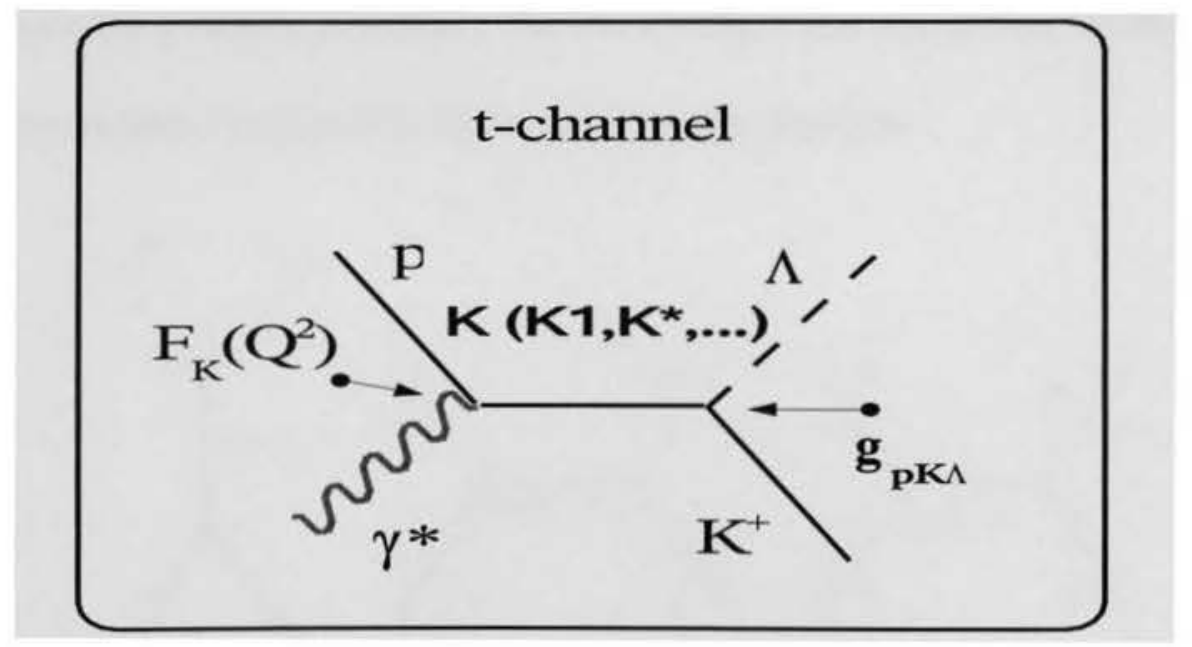

Figure 2: Tree level Feynman diagram in $H\left(e, e^{\prime} K+\right) Y$ reaction, $t$ channel exchange; kaon form factor and coupling constant indicated at vertexes. 
The last two terms, $M^{5}$ and $M^{6}$, of the bilinear matrices are specific for electroproduction processes (they take into account the longitudinal spin degrees of freedom of the virtual photon). The elementary transition amplitudes, $A_{i}$, are expressed as functions of the Mandelstam variables for each of the different channels contributing to the kaon electroproduction process. The transition amplitudes $A_{i}$ are proportional with the kaon form factor $F_{K}\left(Q^{2}\right)$ (see Fig. 2):

$$
A_{i}\left(q^{2}, s, t, u\right) \propto F_{K}\left(Q^{2}\right) \cdot g_{p K \Lambda}
$$

where $g_{p K \Lambda}$ is the coupling constant (considering the $t$ channel exchange from Fig. 3). From the expressions of the transition amplitudes and kaon electroproduction data, it is theoretically possible to extract the contribution and the extent of the different reaction mechanisms responsible for kaon electroproduction.

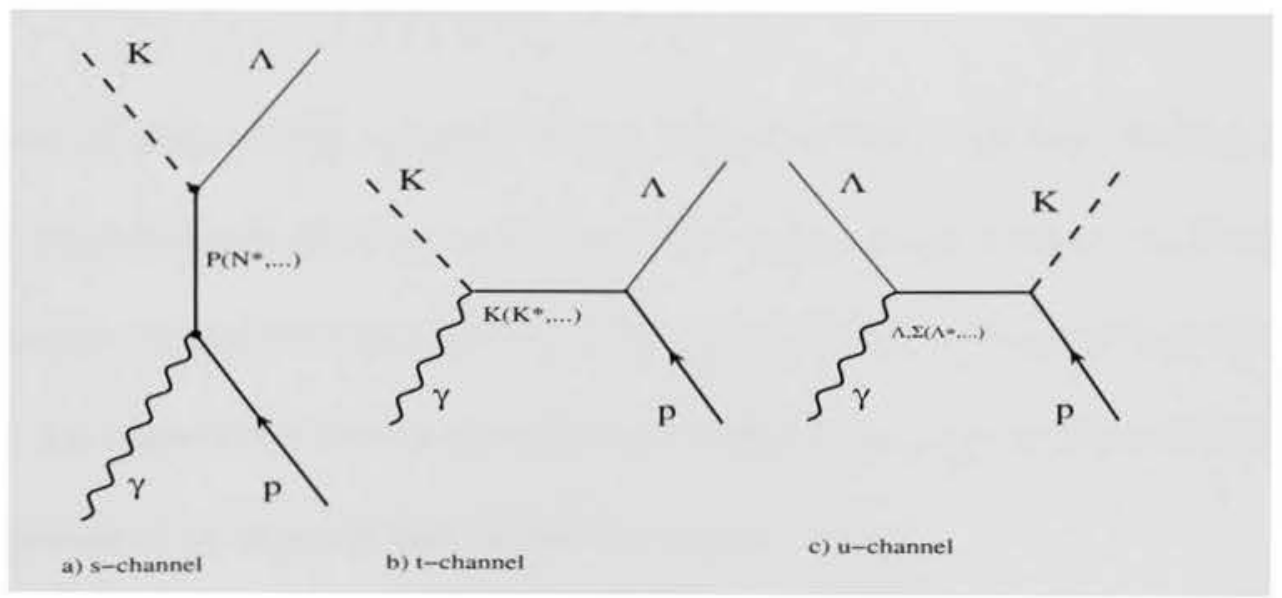

Figure 3: Tree level Feynman diagrams in $H\left(e, e^{\prime} K+\right) Y$ reaction [PRO98]. In hadronic language the production mechanism can happen through $s, t$, or $u$ channels. 
The five-fold differential cross section formula, from Eq. 1, for kaon electroproduction by virtual photons, can be rewritten as:

$$
\frac{d^{5} \sigma}{d E^{\prime} d \Omega_{e^{\prime}} d \Omega_{K}}=\Gamma \cdot \frac{d \sigma_{v}}{d \Omega_{k}}
$$

where $\frac{d \sigma_{v}}{d \Omega_{K}}$ is the kaon virtual photoproduction cross section and $\Gamma$ the virtual photon flux. The virtual photon flux is a function of the four-momentum transfer $Q^{2}$, the incident and outgoing electron's energy and the virtual photon polarization parameter, $\epsilon$ :

$$
\Gamma=\frac{\alpha}{2 \pi^{2}} \frac{E^{\prime}}{E} \frac{s-M_{P}^{2}}{2 M_{P} Q^{2}} \frac{1}{1-\epsilon}
$$

with $\alpha=\frac{1}{137}$ being the fine structure constant (electromagnetic coupling constant) $E^{\prime}$ the final electron energy. The quantity

$$
s=M_{P}^{2}+2 \cdot M_{P} \cdot \nu-Q^{2}
$$

is the center of mass energy squared (of the virtual photon - proton system) and $\nu=E-E^{\prime}$ represents the electron energy loss and $M_{P}$ the proton's mass. In the most general case the virtual photoproduction cross section (in the case of an unpolarized target and the coincidence detection of the final state hadron and scattered electron) can be represented in terms of four invariant cross sections as:

$$
\frac{d \sigma_{v}}{d \Omega_{K}}=\frac{d \sigma_{T}}{d \Omega_{K}}+\epsilon \cdot \frac{d \sigma_{L}}{d \Omega_{K}}+\epsilon \cdot \frac{d \sigma_{T T}}{d \Omega_{K}} \cdot \cos (2 \phi)+\sqrt{2 \epsilon_{L}(\epsilon+1)} \cdot \frac{d \sigma_{L T}}{d \Omega_{K}} \cdot \cos \phi
$$

where $\sigma_{T T}$ is the cross section due to the interference between transversely polarized virtual photons, $\sigma_{T}$ is the cross section due to transversely polarized virtual photons, 


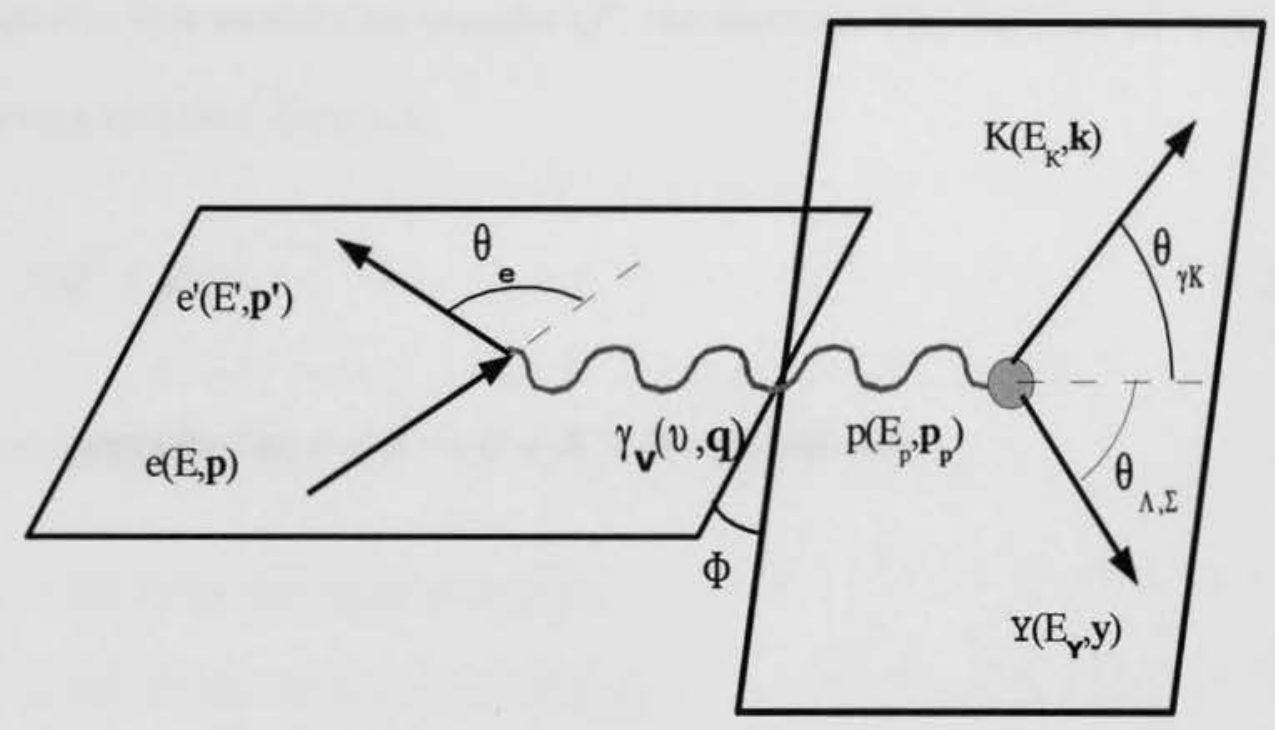

Scattering plane and reaction plane in kaon electroproduction

$$
\mathrm{e}+\mathrm{p} \longrightarrow \mathrm{e}^{\prime}+\mathrm{K}+\mathrm{Y}(\Lambda, \Sigma)
$$

Figure 4: Studied Kaon electroproduction reaction, $H\left(e, e^{\prime} K+\right) Y$ in E98-108 experiment at JLab; the bold notation is used to represent vectors ( $\mathbf{p}$ stands for $\vec{p})$.

$\sigma_{L}$ is due to longitudinally polarized virtual photons, $\sigma_{L T}$ is due to the interference between longitudinally and transversely polarized virtual photons and $\phi$ is the angle between the leptonic (scattering) plane and the hadronic (reaction) plane (see Fig. 4). 
The $\sigma_{L}, \sigma_{T}, \sigma_{T T}, \sigma_{L T}$ virtual photoproduction cross sections are a function of the electromagnetic four-momentum transfer $Q^{2}$, the hadronic four-momentum transfer $t$, and Lorentz invariant energy $s$ :

$$
\sigma=f\left(Q^{2}, t, s\right)
$$

The four momenta for the $e+N \rightarrow e^{\prime}+K+X$ reaction are:

$$
\begin{aligned}
e & =(E, \vec{e}) \text { for the incident electron } \\
e^{\prime} & =\left(E^{\prime}, \overrightarrow{e^{\prime}}\right) \text { for the scattered electron } \\
N & =\left(E_{P}=M_{P}, \vec{p}_{P}=\overrightarrow{0}\right) \text { for the target nucleon in the lab frame } \\
K & =\left(E_{K}, \vec{k}\right) \text { for the produced kaon } \\
Y & =\left(E_{Y}, \vec{y}\right) \text { for the unobserved residual system. }
\end{aligned}
$$

The Lorentz invariants we deal with and other kinematic variables are defined below:

$$
\begin{aligned}
s & =\left(\gamma_{V}+N\right)^{2}=W^{2} \quad \text { the center of mass energy } \\
t & =\left(\gamma_{V}-K\right)^{2} \quad \text { the Mandelstam t variable } \\
M_{\Lambda}^{2} & =\left(e-e^{\prime}+N-K\right)^{2} \quad \text { the missing mass } \\
\gamma_{V}^{2} & =\left(e-e^{\prime}\right)^{2}=-Q^{2} \quad \text { the mass of the virtual photon } \\
\nu & =E-E^{\prime} \quad \text { the energy transfer } \\
\epsilon & =\left[1+2 \frac{\nu^{2}+Q^{2}}{Q^{2}} \tan ^{2} \frac{\theta_{e}}{2}\right]^{-1} \quad \text { the polarization of the virtual photon } \\
\epsilon_{L} & =\frac{Q^{2}}{\nu^{2}} \epsilon \quad \text { the longitudinal polarization. }
\end{aligned}
$$




\subsection{Kaon electroproduction: Models and previous data}

In order to understand electroproduction processes of strangeness - particles having a strange quark in their composition - semi-phenomenological descriptions in terms of mesons and baryons are used. These hadronic field models do not contain the quark degrees of freedom explicitly, but they provide an effective description of the electroproduction dynamics up to an invariant mass, $W$, of $2(\mathrm{GeV} / \mathrm{c})^{2}$. The semiphenomenological parameters of the theory (strong coupling constants, transition magnetic moments) are fit to the available photoproduction and electroproduction data using transition amplitudes based on the tree level (only lowest order in the perturbative expansion are considered) Feynman diagrams method (see Fig. 3). There are experiments in which the polarization of the target and/or the beam are measured in order to determine the electroproduction mechanism. While these experimental results indicate problems with the models, polarization data have yet to be included in the model fits.

A short overview of the electroproduction models will be given. The models were selected out of those suitable for describing kaon electroproduction in the E98-108 experiments' energy range. The isobaric models were chosen since their description is in good agreement [BYC03] with the data in the energy range $E_{\gamma}^{L a b}=0.9-2.5$ $\mathrm{GeV}$. The latest isobaric models chosen are the Saclay-Lyon (SLA) [DAV96] [MIZ98] model, the Kaon-Maid model [MAR03] and two models, M2(WAA) and M2(WWW), developed by Petr Bydžovský and Miloslav Sotona [BYD04] from the Nuclear Physics 
Institute, $\breve{R} e \breve{z}$ near Prague, Czech Republic, from fits to the CLAS photoproduction data [MCN04].

In the isobaric models, the transition amplitudes, $A_{i}$ from Eqs. 3 and 4 , are derived using the Feynman diagram method (in the tree level approximation - Fig. 3), from an effective hadronic lagrangian. Both the Born diagrams, (in which the proton, a $\Lambda$ and a $\Sigma$ are exchanged depending on $s, t$ or $u$ channel) as well as resonant diagrams (in which excited nucleons, hyperons and kaons are exchanged) contribute to the invariant amplitude in the isobaric models. The corresponding coupling constants are introduced as free parameters in the isobaric models. In order to narrow down the suitable isobaric models, crossing symmetry and SU(3) symmetry conditions are imposed onto the models (for example an isobaric model is required to describe equally well the radiative capture of $K^{-}$on the proton with a $\Lambda$ on a final state and $\Lambda$ photoproduction). The SU(3) symmetry condition imposes limits on the values allowed for the coupling constants.

The models developed by P. Bydžovský and M. Sotona [BYS03] use a prescription by Gari and Krümpelmann [KR $\ddot{U} 92]$ regarding Extended Vector Dominance for the form factors of exchanged baryons-protons, $\Lambda, N^{*}$ - and Vector Meson Dominance for the form factors of mesons exchanged in the t-channel $\left(K^{+}\right.$[WIL92], $K^{*}, K_{1}$ [ADE88]).

In the Saclay-Lyon (SLA) model [DAV96] [MIZ98], besides the baryon resonances with spin $1 / 2$ included by other models, the nucleonic resonances up to spin $3 / 2$ (N7 
at $1720 \mathrm{MeV}$ ) were also included but the nucleonic resonances with spin $5 / 2$ were not included. The free parameters (coupling constants and form factors) in the SaclayLyon model were extracted from fits to kaon photoproduction data in the energy range up to $\approx 2 . \mathrm{GeV}$ (and to the data from the radiative capture of $K^{-}$on the proton [BYC03]).

In the case of the Regge Model [GUI00], the electromagnetic form factors in the photon vertexes were added to fit the data from the E93-018 experiment at JLab [NIC98]. The form used for the electromagnetic form factor is:

$$
F\left(Q^{2}\right)=\frac{\Lambda_{K, K *}^{2}}{\Lambda_{K, K *}^{2}+Q^{2}}
$$

where the factor $\Lambda_{K, K *}=1.5 \mathrm{GeV} / \mathrm{c}^{2}$.

The main assumption behind one of the models (the Regge model [GU100]) used to compare the E98-108 data with (see Section 5 ) is that at high $W$ the $t$-channel diagram of Fig. 3 has the kaon as the meson exchanged (kaon pole diagram) and that this diagram dominates all the other diagrams. If one isolates the $t$-channel contribution, the kaon form factor, $F_{K}\left(Q^{2}\right)$, can be extracted. To be able to separate the $t$-channel contribution it is necessary to perform a separation of the response functions (longitudinal and transverse) and a comparison of the separated responses with available electroproduction models. For illustration purposes only, considering the kaon electroproduction in the quark model (see Fig. 5), and isolating the $t$ channel contribution, the initial momentum of the strange quark can be sampled, and kaon's momentum can be directly related to the initial momentum of the strange 
quark (this is valid in the experiment's energy range when a low particle multiplicity is expected). The final state contains only two particles (the kaon and the hyperon) and the kaon's transverse momentum (with respect to the three momentum transfer) is equal with the transverse momentum of the struck quark. The assumption of $t$ -

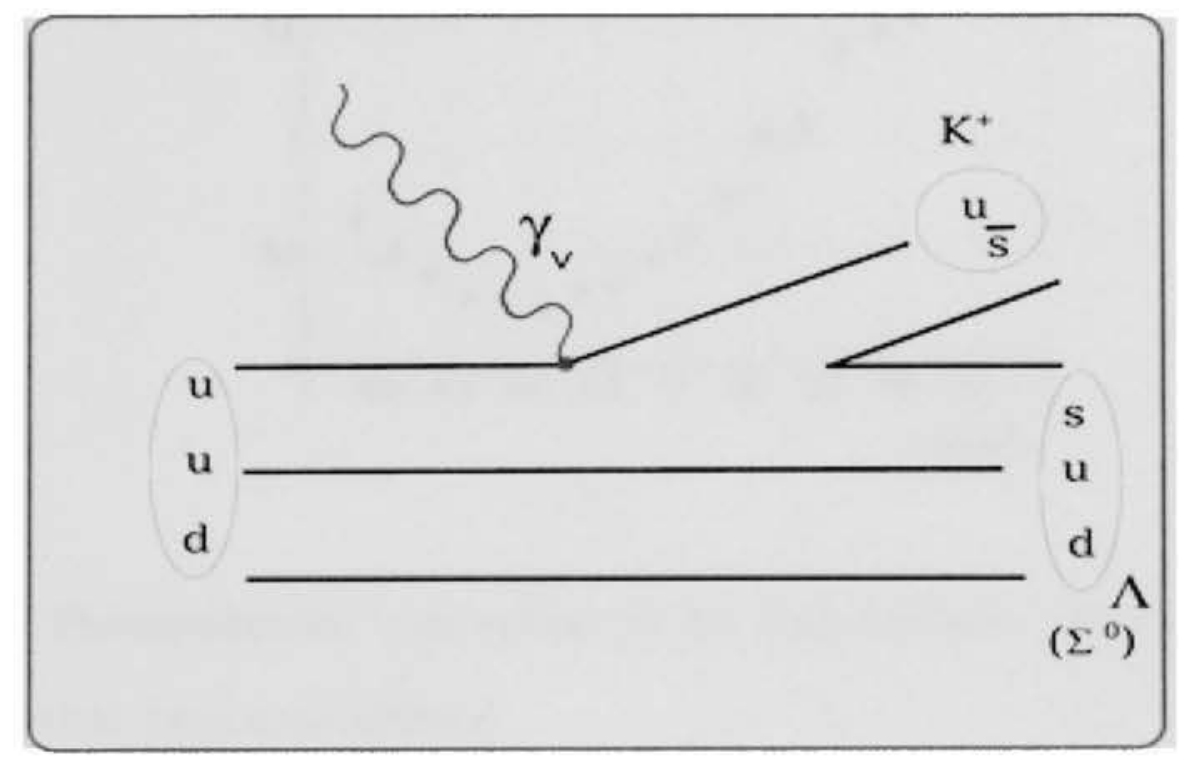

Figure 5: Qualitative description of the kaon electroproduction.

channel dominance is justified by previous data from photoproduction [MCN04] and electroproduction [NAS04] experiments. In Fig. 6, the plot shows the center-of-mass cross section versus $\cos \left(\theta_{K}^{C M}\right)$ from photoproduction. The $\Lambda$ photoproduction cross section exhibits an increasing trend (peak) at forward angles at similar $W$ values as those listed for the E98-108 experiment (see Table 5.1 in Appendix E). This photoproduction data is consistent with a $t$-channel dominance. This is in contrast to the cross section for $\Sigma^{0}$ production, which is not peaked at forward angles and exhibits an $s$-channel dominance. Further support for the $t$-channel dominance in $\Lambda$ 


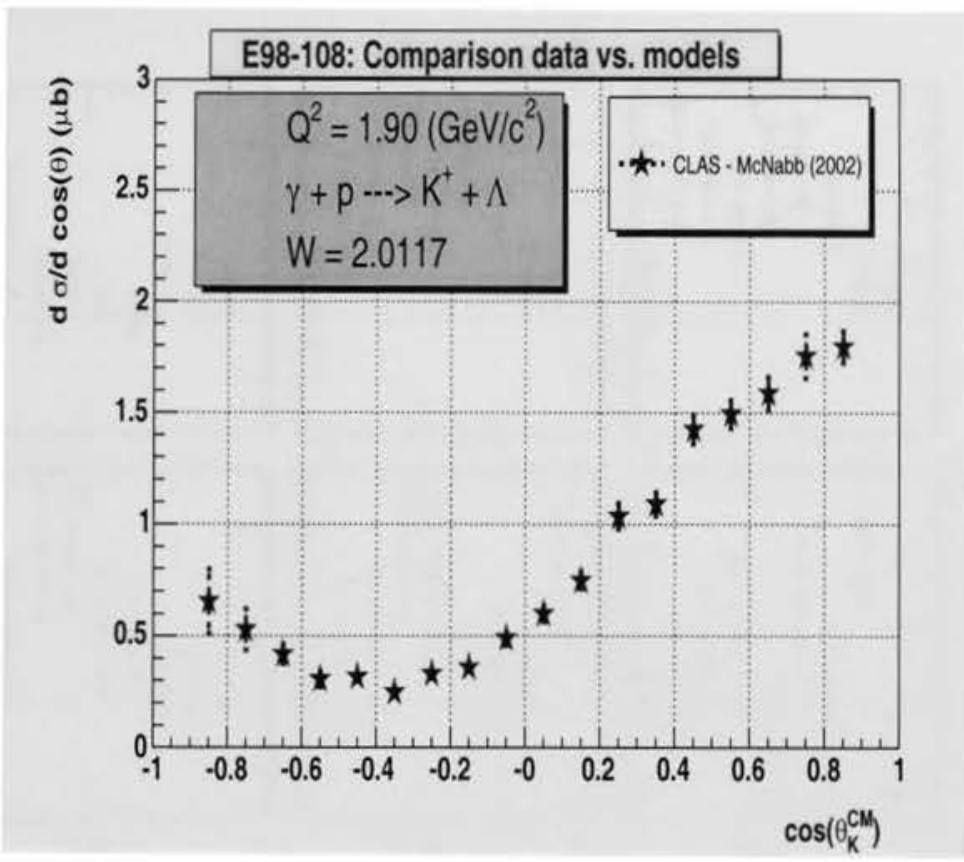

Figure 6: Photoproduction cross section (in the center-of-mass) versus $\cos \left(\theta_{K}^{C M}\right)$, $W=2.017 \mathrm{GeV}$. Data from [MCN04].

electroproduction (at forward angles and $W$ values similar to those of the E98-108 experiment) can be extracted from measurements of the polarized structure functions in the exclusive $p\left(\vec{e}, e^{\prime} K^{+}\right) \Lambda$ reaction (Fig 7) experiments, performed in Hall-B at JLab [NAS04]. From these measurements one can conclude that at high $W$ (above $1.9 \mathrm{GeV})$, there is no indication of a large interference structure function, $\sigma_{L T^{\prime}}$, at forward angles [RAU05]. The small $\sigma_{L T^{\prime}}$ can be interpreted as the dominance of a single $t$-channel mechanism or, if more production channels are contributing, they contribute in a destructive manner (cancelling each others' contribution). The data in Fig. 7 is from [NAS04] and indicates that a single reaction channel is the dominant 


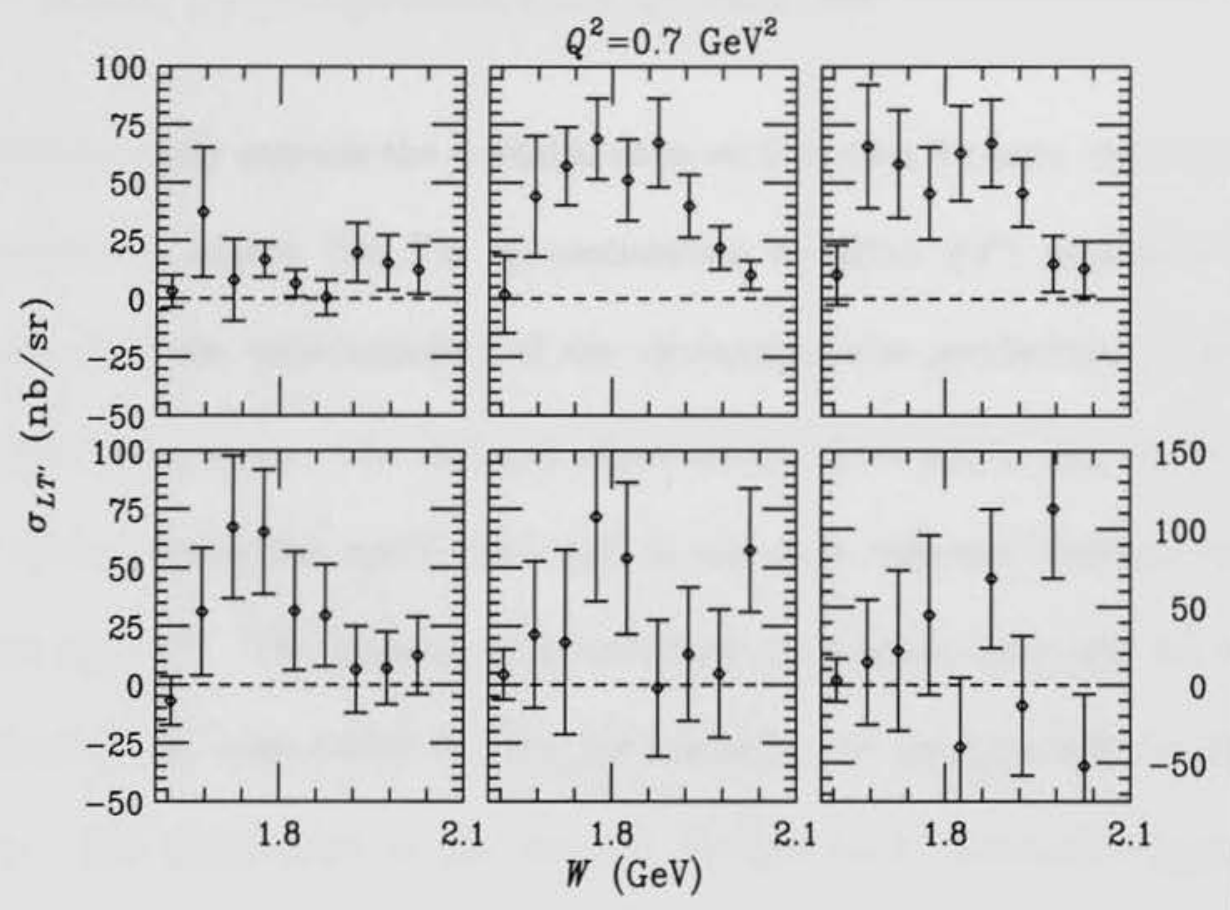

Figure 7: Polarized LT' interference response function versus $W$ from electroproduction data; $W=1.625-1.975 \mathrm{GeV}, Q^{2}=0.7 \mathrm{GeV}^{2}$; figure from [NAS04].

mechanism. However at lower $W$ values of $1.7-1.8 \mathrm{GeV}$, the observed polarizations are indeed large (compared to higher $W$ values) indicating an interference does occur between two or more production channels. These recent measurements are experimental evidence for the theoretical suggestion that at high $W$, the $t$-channel dominates. 


\subsection{Kaon electroproduction in E98-108}

The present study extends the available cross section data for kaon electroproduction to unexplored, higher (see Fig. 8) momentum transfers $\left(Q^{2}\right)$ providing the basis for improving our understanding of the electromagnetic production of strangeness at subnucleonic level. The E98-108 experimental data fills a gap in the existent kaon electroproduction world data and is the only response function separation at such high $Q^{2}$. The kaon electroproduction experiment may give an insight to the mechanisms responsible for the hadronization of partons into an observable meson. The parameters of the existent models used to describe kaon electroand photoproduction are extracted from available data using transition amplitudes derived from Feynman diagrams (see Fig. 3) and from hadronic field theory. The fitting parameters are not unique due partially to the limited kinematical region of the published data. Also the accuracy of the unseparated cross sections so far is at the level of $10 \%$.

The present study, a Rosenbluth separation of the response functions will put additional constraints on the models and on the fitting parameters (the coupling constants) and the accuracy level will improve the available kaon electroproduction data. Also, the kinematical region covered will be broadened with the present data. The primary study is the determination of the differential cross sections for the kaon electroproduction reaction and performing a Rosenbluth separation of the longitudinal and transverse cross sections. In order to do that, the missing 


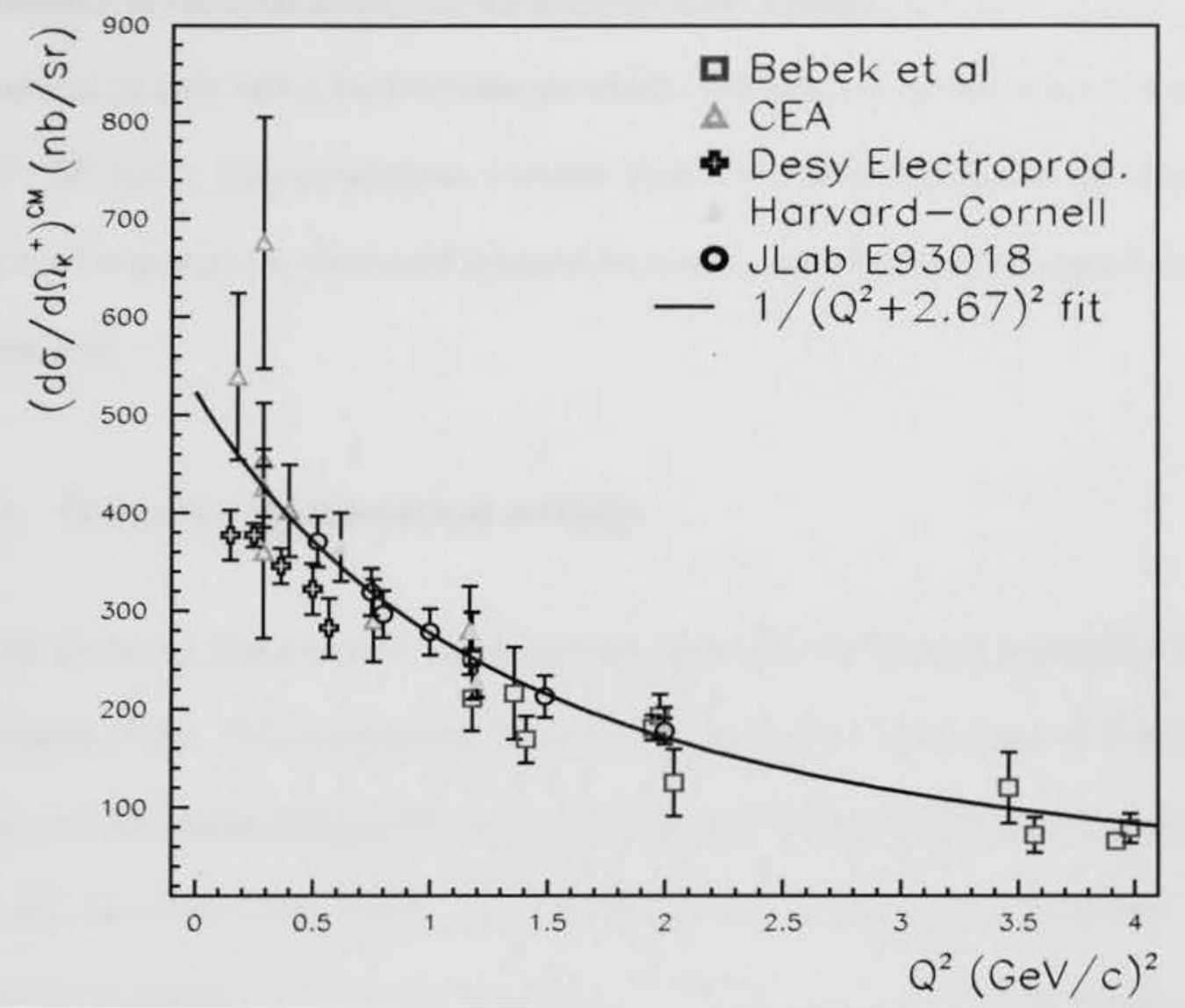

Figure 8: Kaon electroproduction: previous world data [NIC98]; Cross sections are extrapolated $[\mathrm{BEB} 77]$ to $\mathrm{W}=2.15 \mathrm{GeV}$.

mass spectra (see Fig. 1) for 20 different kinematics (presented in Table 5.1) were produced and analyzed. The separation of the longitudinal and transverse response functions (the separation was studied for the $\Lambda$ hyperon only - see Section 4.3) will give information on the electromagnetic substructure of the kaon as well as shed light on the electroproduction mechanism and provide the basis for further studies describing it in terms of the transition to quark degrees of freedom. Finally the 
dependence of the cross section on the hadronic four momentum transfer, $t$, provides theoretical models with a considerable set of data covering a large kinematical region in $W, Q^{2}$ and $t$ (four-momentum transfer from the virtual photon to the kaon), allowing constraints on the model parameters used to describe the electroproduction mechanisms.

\subsubsection{Overview of kinematical settings}

In this study the cross section has been determined for 20 different kinematics (see kinematics Table 5.1 in appendix E). The measurements were taken at different values of $\epsilon$ (the polarization of the virtual photon), the same four-momentum transfer $Q^{2}$, and the same total center of mass energy, $W$, in order to accomplish the Rosenbluth separation of the cross section. The kaon was measured along the direction of the virtual photon with a complete coverage in $\phi$, the angle between the leptonic (scattering) plane and the hadronic (reaction) plane (see Fig. 4). The coverage in $\phi$ ensures that the interference terms of the cross sections (in Eq. 6) cancel out, when performing an integration over $\phi$ from 0 to $2 \pi$, leaving only the longitudinal and the transverse terms to contribute to the cross section:

$$
\sigma_{C M}=\sigma_{T}+\epsilon \cdot \sigma_{L}
$$

Plotting the center of mass cross section as a function of the polarization of the virtual photon (keeping simultaneously constant $Q^{2}, W$, and $t$ and varying the electron and 
the kaon scattering angles) the longitudinal and the transverse cross section are extracted by determining the slope and, respectively, the intercept of the graph. 


\section{Instrumentation}

\subsection{Overview of Jefferson Lab; Accelerator and Hall A beam line}

The experiment was performed at the Thomas Jefferson National Accelerator Facility (Jefferson Lab) in Newport News, Virginia, a superconducting, continuous-wave electron accelerator that delivers electron beams to three experimental halls. The facility can deliver beams up to an energy of $6 \mathrm{GeV}$. A laser in the infrared region is used to knock-out polarized electrons from a GaAs photocatode generating the electron beam. Inside the injector, the electron beam is accelerated to $45 \mathrm{MeV}$ and delivered afterwards to a linear accelerator. Each of the two linear accelerators, or linacs, accelerate the electrons up to $0.6 \mathrm{GeV}$. After passing through the first linac the electrons are focused and separated according to their energies by a magnetic field in the recirculation arcs and redirected to the second linac (see Fig. 9). Each linear accelerator consists of twenty cryomodules. Each cryomodule contains eight superconducting niobium cavities cooled down to a temperature of $2 \mathrm{~K}$. The cavities are driven by $1.497 \mathrm{GHz}$ radio frequency waves. The frequency of the radio waves, $\nu_{R F}$, was calculated such that the periodicity of the elliptical cavities is equal to the distance between the electron packets: $\frac{c}{\nu_{R F}}=20.04 \mathrm{~cm}$. The range of the beam current is from $1 \mu \mathrm{A}$ to $190 \mu \mathrm{A}$. This process of circulating the electron beam can be repeated up to 5 times. When the desired energy of the beam is attained, the beam 
tabulated in Table 5.1 in Appendix E. In the MySQL database described in Appendix I, one of the parameters that characterize each run is the Tiefenbach energy which is contained in the data stream. The Tiefenbach energy is calibrated using the arc energy measurements and is calculated by using the beam position monitors.

\subsection{Beam current monitor}

The beam current monitoring (BCM) utilizes a non-invasive method which relies on two beam current monitors, and is designed to provide a direct measurement and absolute calibration of the beam's current. The beam monitors consist of an Unser monitor and two radio frequency cavities (cylindrical wave guides), enclosed in a box (which acts as a shield for the magnetic field) together with a data acquisition system. The beam current monitors are calibrated by circulating a known current through a wire located inside the electron beam pipe. The signals from the beam current monitors are used to determine the beam charge during a run having known the acquisition time for each run. The BCM final signals are contained in the EPICS data stream.

The graph of the ratio of the two charge readings, unamplified and amplified, (Charge_1x and Charge_3x) is presented in Fig. 10. 


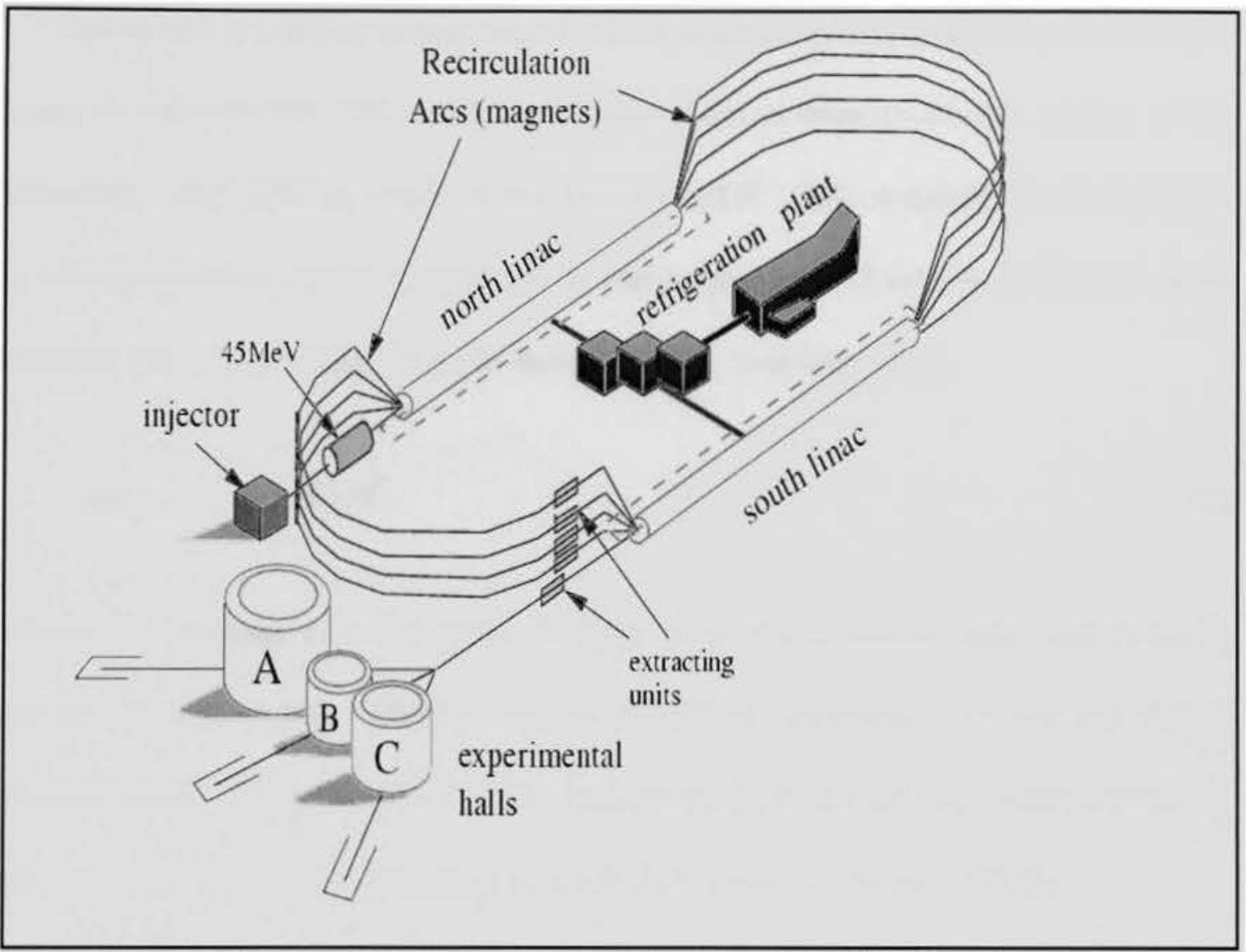

Figure 9: The accelerator at Jefferson Lab.[HWEBA].

is delivered to one of the three experimental halls. For the E98-108 experiment the range in energy was $3.4006-5.7544 \mathrm{GeV}$.

\subsection{Beam energy measurement during E98-108}

Knowledge of the energy of the beam is essential in determining and calculating the kinematic variables of the particles produced in a nuclear reaction. Two independent methods of measuring the beam energy were used for the E98-108 experiment. 
One is the arc energy measurement, which is performed by deflecting the electron beam in a known magnetic field; the measurement is done in the arc section of the beamline. The bending angle of the beam is $34.3^{\circ}$. The momentum of the beam is calculated knowing the magnitude of the magnetic field and the net bend angle through the arc by using the path integral of the magnetic field:

$$
p_{\text {Beam }}=\frac{c}{\theta} \cdot \int \vec{B} \cdot d \vec{l}
$$

where the constant $c$ is the speed of light, $\vec{B}$ is the magnetic field and $d l$ is the electrons' path length. The systematic (absolute) uncertainty for the arc energy method ranges from $6.8 \times 10^{-5} \mathrm{GeV}$, corresponding to an incident energy of $4 \mathrm{GeV}$ to $1.2 \times 10^{-4} \mathrm{GeV}$, corresponding to a $0.5 \mathrm{GeV}$ incident energy [ALC04].

The second method to measure the beam energy is based on the elastic scattering of the electron beam off a proton target, or the $e p$ method. The elastic scattering method can achieve a precision in the relative energy $\frac{\delta E \_b e a m}{E \_b e a m}$ of $2 \times 10^{-4}$ [ALC04]. The energy of the beam is determined in the elastic scattering method by measuring the angle of the scattered electron and the angle of the recoil proton using two-body kinematics:

$$
E=M_{p} \frac{\cos \left(\theta_{e}\right)+\frac{\sin \left(\theta_{e}\right)}{\tan \left(\theta_{p}\right)}-1}{1-\cos \left(\theta_{p}\right)}
$$

The agreement between the beam energy measured by the two methods, the elastic scattering and the arc energy, is $\leq 3 \times 10^{-4}$ (relative uncertainty). The incident beam energies used to determine the missing mass for the E98-108 experiment are 


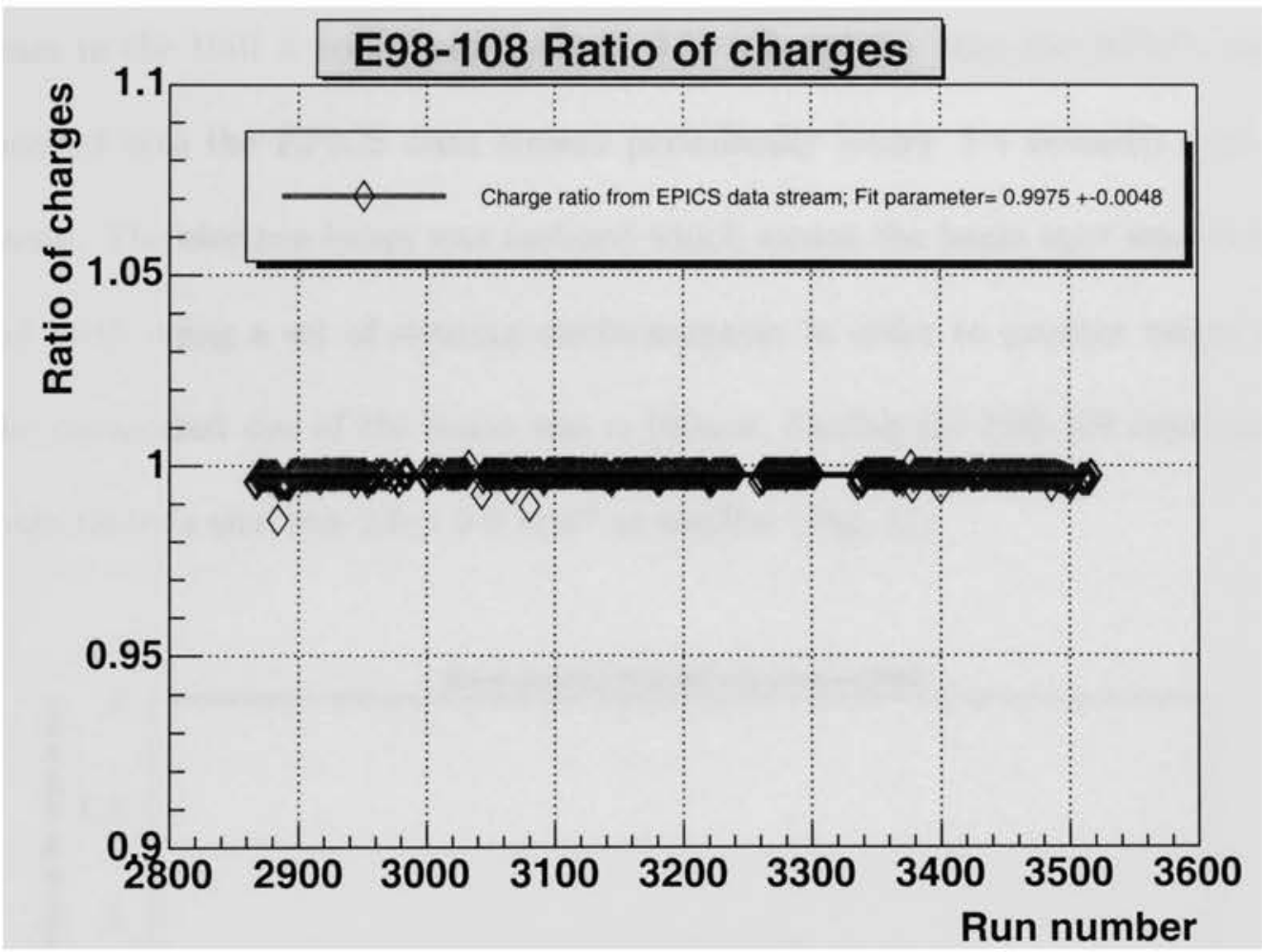

Figure 10: Ratio of the charge measurements as extracted from the EPICS datastream for the E98-108 experiment.

\subsection{Beam position monitor - BPM}

The position of the beam, horizontally and vertically, along the beamline is monitored by two devices located upstream of the target (1.3 meters and 7.5 meters away from the target). The position of the beam is extracted from eight readings, one from each of the BPM's eight antennas. The BPM's are calibrated against invasive wire scanners located near the monitors. The wire scanners (harp scanners) provide a precise $(50 \mu)$ measurement of the beam's position and of the beam's profile. The 
scanners are surveyed on a regular basis and determine the absolute position of the beam in the Hall A coordinate system. The information that the BPM's provide is encoded into the EPICS data stream periodically (every 3-4 seconds) with a time stamp. The electron beam was rastered which means the beam spot was swept back and forth using a set of steering electromagnets in order to prevent target boiling. The unrastered size of the beam was $\approx 100 \mu \mathrm{m}$. During the E98-108 experiment the beam raster's size was $2.0 \times 2.0 \mathrm{~mm}^{2}$ or smaller (Fig. 11).

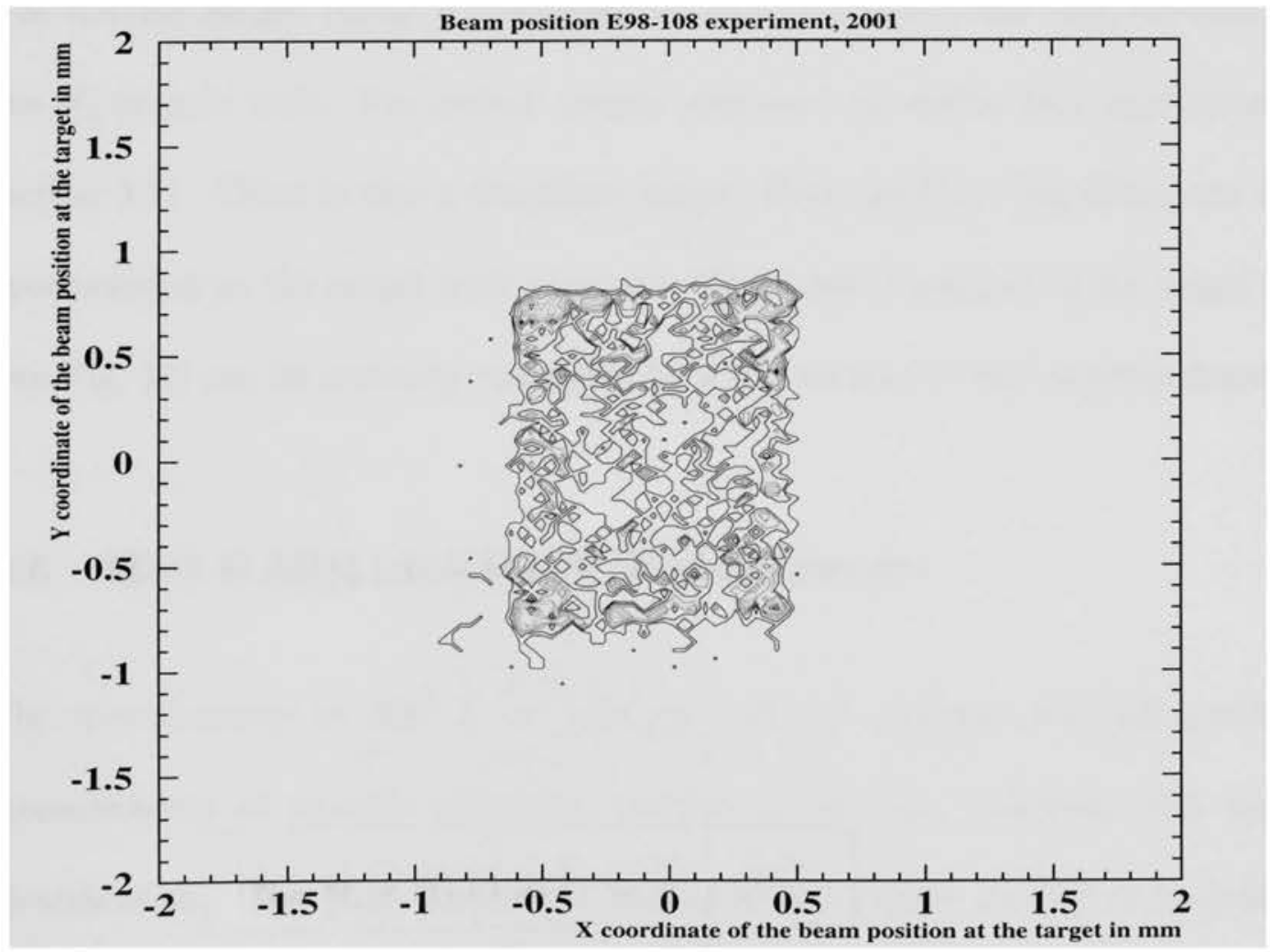

Figure 11: Rastered beam size on target for E98-108, 2001. 


\subsection{Target configuration}

We have used a liquid hydrogen target, $\mathrm{LH}_{2}$, for the entire kaon electroproduction experiment, as well as special solid targets used for optics studies (see section 3.1). The cryogenic target is mounted inside a scattering chamber together with cooling systems, gas handling devices, temperature and pressure sensors and subsystems for target motion and target control. The liquid hydrogen target and solid carbon targets (as well as a dummy target) are attached to a vertical ladder (see Fig. 13). The dummy target, made of aluminum, is used to subtract the contributions from the $H_{2}$ target's walls. The carbon targets were used for optics data acquisition (see section 3.1). There is also a beryllium target, $\mathrm{BeO}$, used for visualizing the beam spot position on the target with a camera. The vertical position of the target stack (see Fig. 13) can be remotely controlled by a $P C$ located in the counting house.

\subsection{Hall A high resolution spectrometers}

The spectrometers in Hall A at Jefferson Lab are designed for high resolution measurements of particle momenta, particle angles and positions, and particle identification. The High Resolution Spectrometers (HRS) in Hall A at Jefferson Lab consist of two magnetic systems and two detector packages [ALC04]. The role of the spectrometers is to select, record, and identify particles emerging from a reaction in the target within a certain phase space in momentum and solid angle which is defined by the acceptance of the spectrometer. Each spectrometer consists 


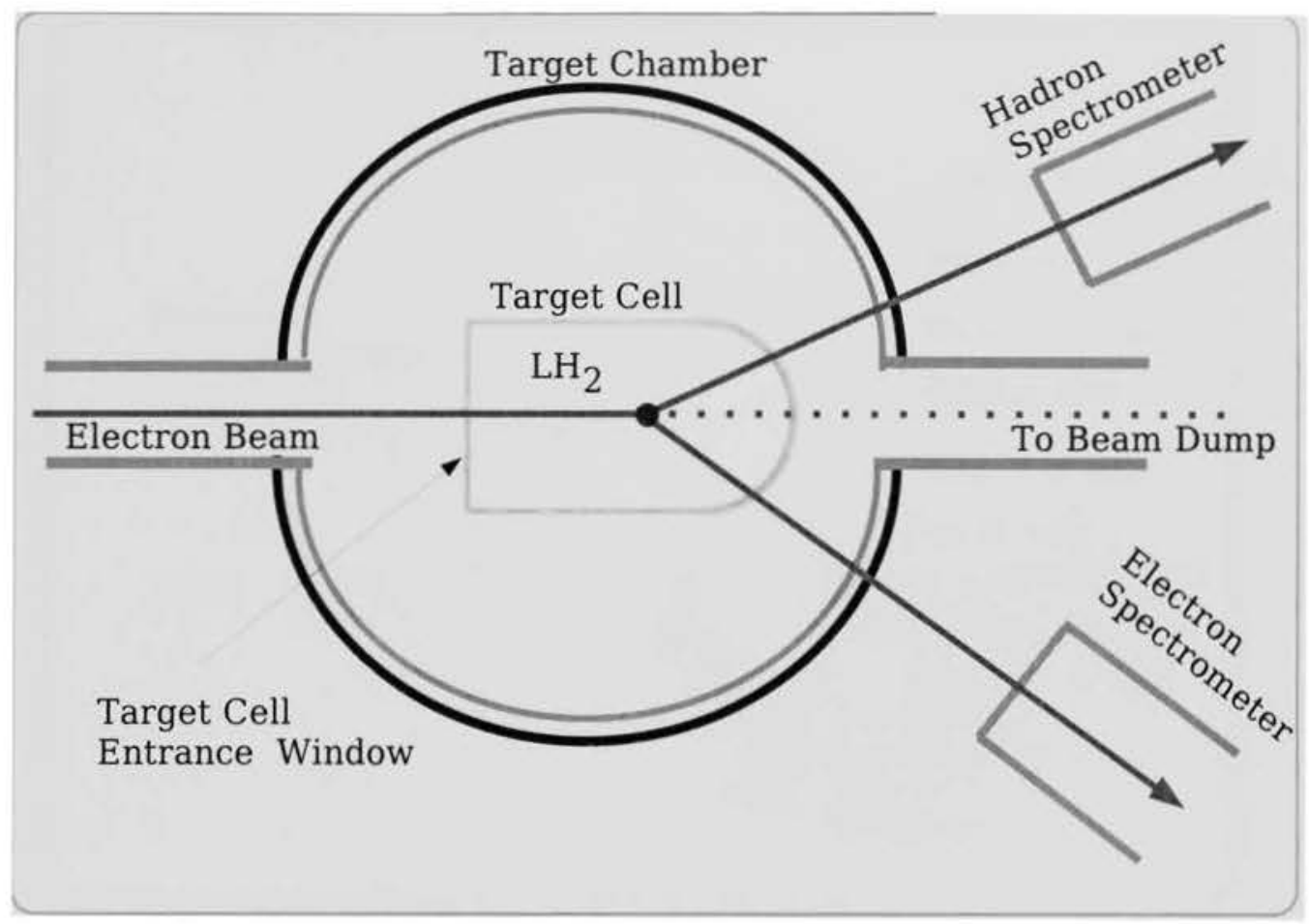

Figure 12: Hall $\mathrm{A} \mathrm{LH}_{2}$ Target aerial view.

of three magnetic quadrupoles and one dipole (see Fig. 14). The configuration of the superconducting magnets is $Q Q D Q$. The dipole acts as the dispersive element and determines the central momentum of the spectrometer. The nominal bending angle of a particle passing through the center of the HRS spectrometers is $45^{\circ}$. The HRS spectrometers can operate in either polarity by changing the current's direction in all of the magnets. The selection of a charged particle depending on its momentum is achieved by the curvature of a charged particle travelling through the dipole magnetic field which is proportional to the particles' momenta. The dipole field can be set to any central momenta value between 0.3 and $4.0 \mathrm{GeV} / \mathrm{c}$. The path length of a particle entering the spectrometer system and following the path of a 


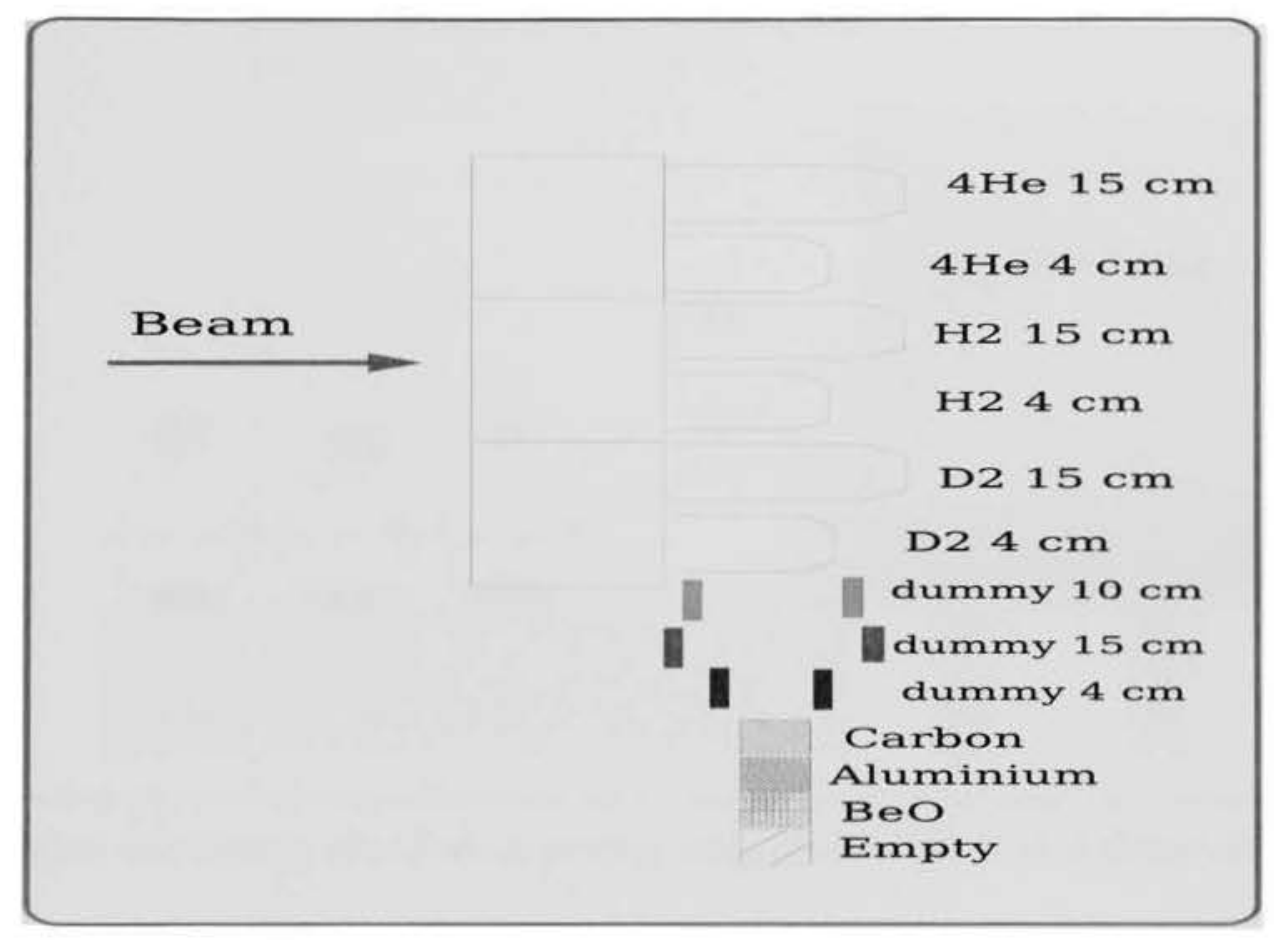

Figure 13: Hall A $\mathrm{LH}_{2}$ Target-side view.

central ray (see dashed line in Fig. 15) is 23.4 meters between the target and the spectrometer's exit window. The particle trajectory -central ray- is the reference for the symmetry plane of the spectrometer system. The quadrupoles determine the transverse and in-plane focusing properties of the spectrometer and, to a large extent, its acceptance. The scattering angle of the incident particles can be varied between $12.5^{\circ}$ and $125.05^{\circ}$ by rotating the entire spectrometer around the Hall $\mathrm{A}$ center. The general characteristics of the Hall A spectrometers [ALC04] are listed in Table 1. 


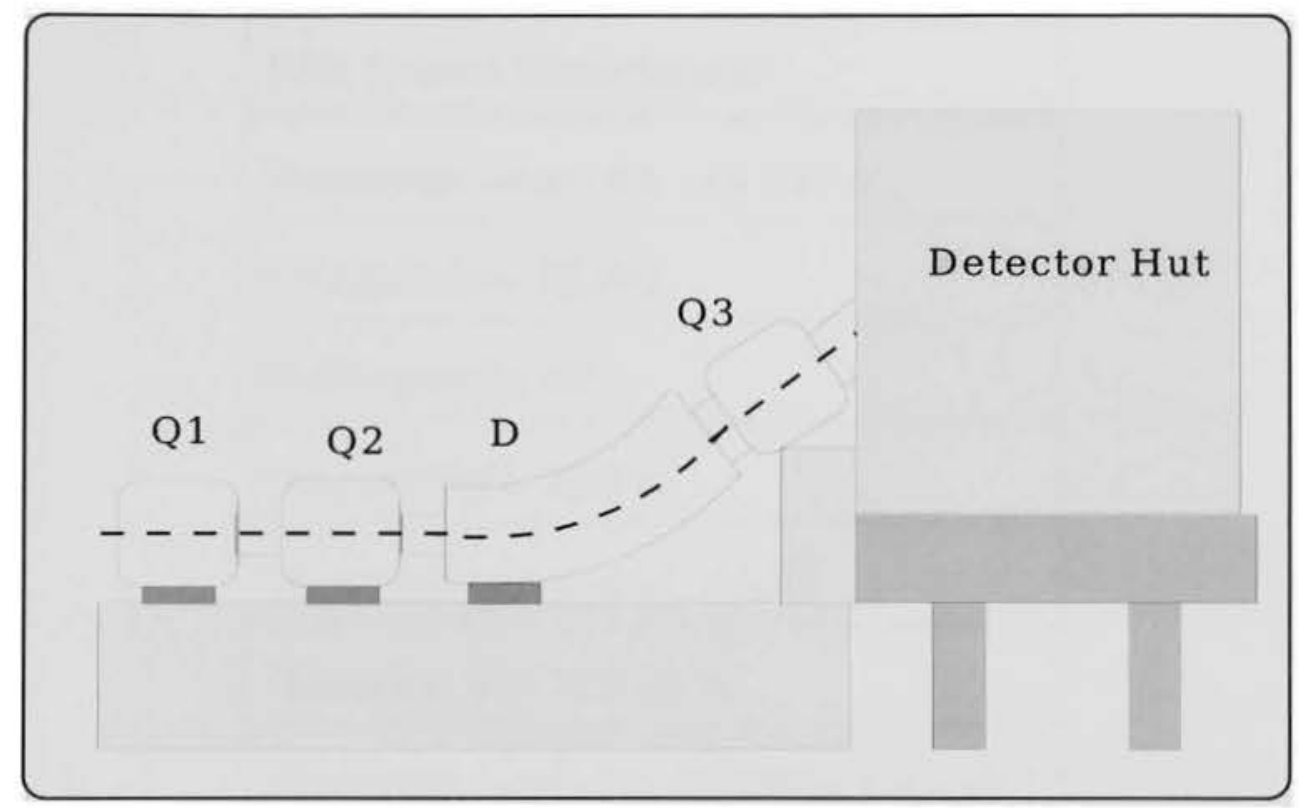

Figure 14: Layout of superconducting magnets in Hall A at Jefferson Lab.

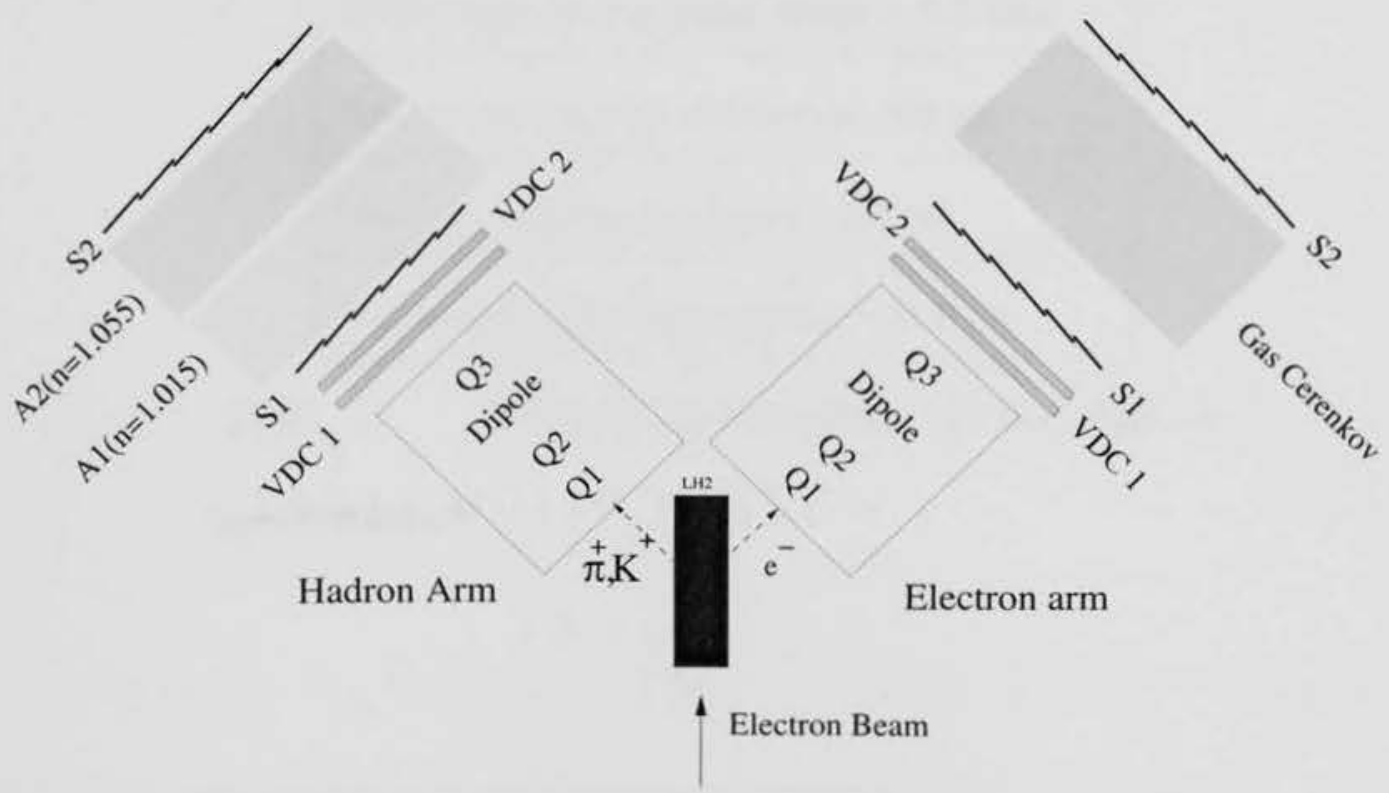

Figure 15: Layout of high resolution spectrometers in Hall A at Jefferson Lab. 


\begin{tabular}{|l|}
\hline HRS General Characteristics \\
\hline Momentum range: $0.3-4.0 \mathrm{GeV} / \mathrm{c}$ \\
\hline Configuration: QQDQ \\
\hline Bending angle: $45^{0}$ \\
\hline Optical length: $23.4 \mathrm{~m}$ \\
\hline Momentum acceptance: $\pm 4.5 \%$ \\
\hline Dispersion (D): $12.4 \mathrm{~cm} \%$ \\
\hline Momentum resolution (FWHM): $2.5 \times 10^{-4}$ \\
\hline Horizontal angular acceptance: $\pm 28 \mathrm{mr}$ \\
\hline Vertical angular acceptance: $\pm 60 \mathrm{mr}$ \\
\hline Solid angle (rectangular shape): $6.7 \mathrm{msr}$ \\
\hline Horizontal angular resolution: $0.6 \mathrm{mr}$ \\
\hline Vertical angular resolution: $2.0 \mathrm{mr}$ \\
\hline Angle determination accuracy: $0.1 \mathrm{mr}$ \\
\hline
\end{tabular}

Table 1: General characteristics of the Hall A spectrometers 
During the E98-108 experiment in Hall A at Jefferson Lab the following Hall A experimental detectors were used:

\begin{tabular}{|l|}
\hline Detector Configurations for E98-108 \\
\hline Left arm (hadron arm) for $\pi^{+}, K^{+}$, protons : \\
\hline$V D C:$ Two Vertical Drift Wire Chambers \\
S1: Trigger scintillator counter \\
A1: 1st Aerogel Cerenkov counter, $n=1.015$ \\
A2: 2nd Aerogel Cerenkov counter, $n=1.0554$ \\
S2: Trigger scintillator counter
\end{tabular}

\begin{tabular}{l} 
Right arm (electron arm) for $e^{-}$and $\pi^{-}:$ \\
\hline$V D C:$ Two Vertical Drift Wire Chambers \\
S1: Trigger scintillator counter \\
GC: Gas Cerenkov counter (long), $n=1.0004$ \\
S2: Trigger scintillator counter \\
PS: Preshower counters \\
TS: Total shower counters
\end{tabular}

The hadron arm detector stack is illustrated in Fig. 15. It consists of two vertical drift chambers (VDCs) that provide a precise measurement of the position and angle of the knockout hadrons at the spectrometer focal plane in the hadron arm. This information is further combined with the knowledge of the spectrometer optics to determine the position and angle of the particles at the target from the known 
information in the focal plane. The electron arm detector stack is similarly illustrated in Fig 15. It also consists of two vertical drift chambers (VDCs) that provide a precise measurement of the position and angle of scattered electrons at the focal plane. This position and angle of electrons is combined with the knowledge of the spectrometer optics to determine the position and angle at the target.

\subsubsection{Scintillator detectors}

Each of the detector stacks (hadron arm and electron arm) contains two planes of trigger scintillators $S 1$ and $S 2$ (see Fig. 16) that were used to generate the trigger and to determine the time of flight for the particles involved in the kaon electroproduction reaction. The plane of each scintillator array is perpendicular to the spectrometer

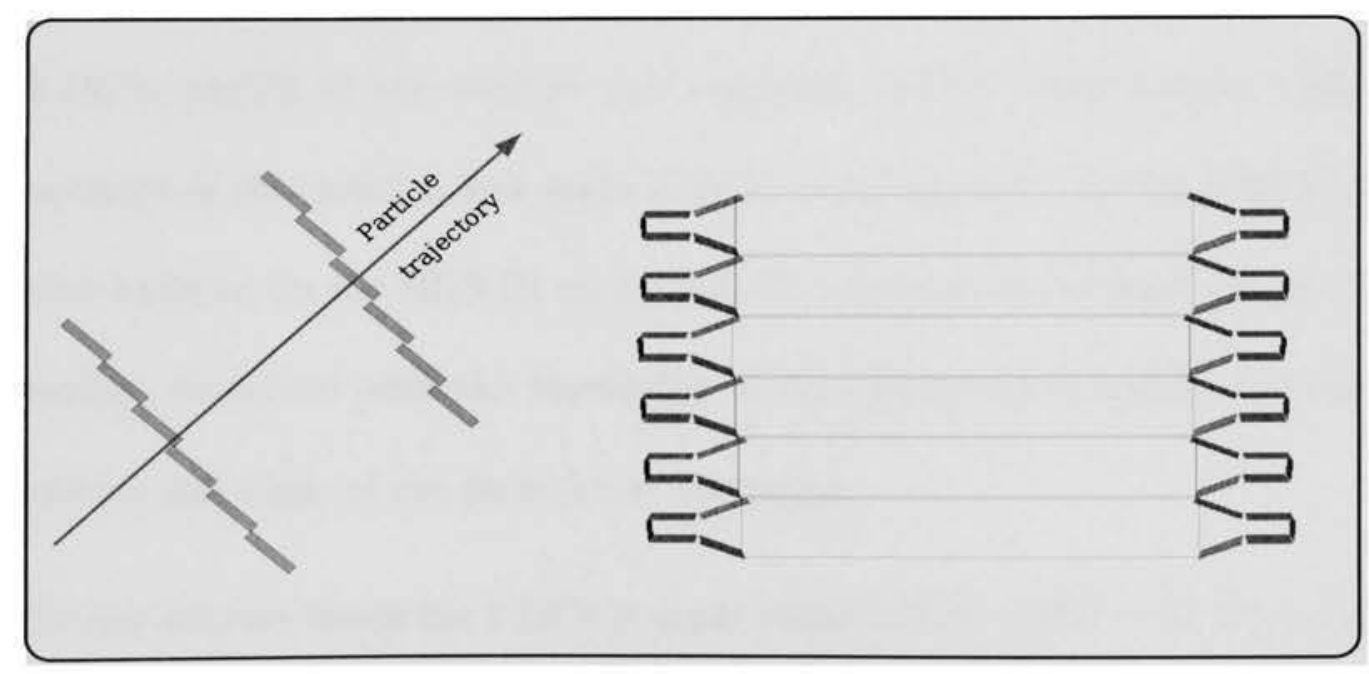

Figure 16: Schematic layout of the scintillator detectors. 
central ray. The time necessary for a particle to travel the distance between the two scintillator planes allows the calculation of the speed of the particle. The first scintillator plane, $S 1$, is located at a distance of 1.5 meters from the center of the first $V D C$ plane $\left(V D C_{U 1}\right)$. The second scintillator plane, $S 2$, is located at a distance of 3.5 meters from the center of the first $V D C$ plane $\left(V D C_{U 1}\right)$. Each of the two scintillator planes consists of six paddles. The active area of the first scintillator plane is about $170 \times 35 \mathrm{~cm}^{2}$ whereas the active area of the second scintillator plane is about $220 \times 54 \mathrm{~cm}^{2}$. The scintillator paddles are made of $5 \mathrm{~mm}$ thick plastic having two PMTs, one at each end. The paddles overlap over a $5 \mathrm{~mm}$ region. The scintillator detectors generate the trigger for the data acquisition system.

\subsubsection{Vertical drift chambers}

The $V D C$ 's consists of two vertical drift chambers (VDCs) that provide a precise measurement of the position and angle of both recoil electrons (in the HRS-E) and knockout hadrons (in the HRS-H) at the respective spectrometer focal planes. This information, combined with the knowledge of the spectrometer optics, determines the position and angle of the particles at the target.

The gas mixture inside the $V D C \mathrm{~s}$ is argon-ethane $(62 \%-38 \%)$. The gas mixture flows continuously with a rate of $10 \mathrm{l}$ /hour. As charged particles pass through the chamber gas in the $V D C$ s, they produce ionization. The electrons produced by ionization are accelerated by the electric field toward the wires, following the shortest 
time path. The drift time is recorded by a Time to Digital Converter (TDC) which is triggered by the wire that fired, and stopped by the scintillator trigger.

The ionized particles, electrons and ions, drift along the direction of the electric field lines defined by the high voltage planes and the signal wires. The potential difference between the cathode planes and the wires is approximately $4 \mathrm{kV}$. The charge is collected in the form of analog pulses on the signal wires. By knowing the drift velocity of the electrons in the argon-ethane mixture, the distance from the wire that fired to the particles trajectory is extracted from the corresponding TDC reading. By calculating the distance for all the wires that fired when a particle passed through the $V D C$ the trajectory of the particle can be determined. The efficiency of the wires (firing efficiency) was typically around $99 \%$ (see $V D C$ efficiency section 3.5).

An incident charged particle making a $45^{\circ}$ angle with respect to the lab plane typically fires five wires. For particles incident at greater angles, the number of wires fired is typically three, whereas for smaller angles the number is typically more. Each of the two vertical drift chambers used for particle tracking is composed of two wire planes, $\mathrm{U}$ and $\mathrm{V}$. The distance between the $\mathrm{U}$ and $\mathrm{V}$ wire planes is $26 \mathrm{~mm}$. The wires of the $\mathrm{V}$ planes are perpendicular to the wires of the $\mathrm{U}$ planes, and all wires make an angle of $45^{\circ}$ with respect to the dispersive and transverse directions. The $V D C \mathrm{~s}$ in each arm are $50 \mathrm{~cm}$ apart from each other. The angular resolution of the $V D C$ (FWHM) is approximately $0.3 \mathrm{mrad}$. The position resolution in the dispersive and transverse directions is $225 \mu \mathrm{m}$ (FWHM). 


\subsection{3 Čerenkov detectors' working principle}

In general when a charged particle crosses a layer of material, three processes can occur: the material's atoms can be ionized, the particle can emit Čerenkov radiation or the particle can generate the emission of transition radiation.

When a charged particle has a velocity greater than the local phase velocity of light, it emits a characteristic cone of radiation (Čerenkov radiation). The charged particle also radiates when crossing suddenly from one medium to another with different optical properties (transition radiation). These two processes are not important from the point of view of the energy loss, but both processes are used in high-energy physics detectors.

The Cerenkov radiation is emitted only if $\beta>1 / n$. The minimal velocity $v_{t}=$ $c / n$ at which Cerenkov emission takes place is called the threshold velocity. Ideally, the threshold Čerenkov counters have a binary response to particles, i.e. they 'fire' (i.e. light is emitted), or not, depending if the incident's particle velocity above or below threshold. Fig. 17 shows the values of the particles' velocities as a function of particles' momentum, together with the threshold velocities for the aerogel detectors used in the E98-108 experiment, aerogels with refractive indexes $n_{A 1}=1.015 \pm 0.002$ and $n_{A 2}=1.055 \pm 0.002$. 


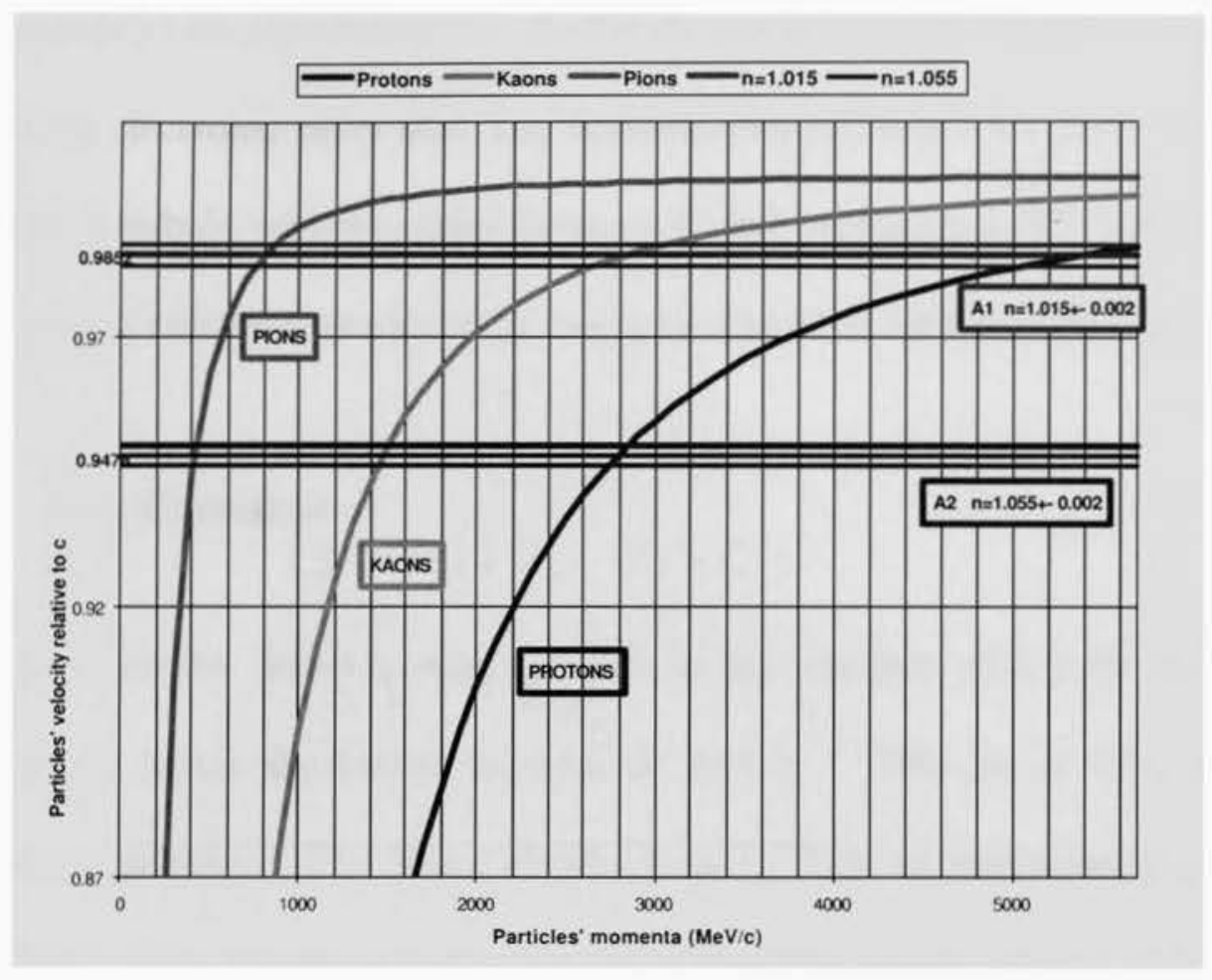

Figure 17: Particle velocities relative to the speed of light as a function of particles' momentum; Threshold velocity for aerogel index $n=1.055 \pm 0.002$ and $n=1.015 \pm$ 0.002 are indicated.

The refractive index of the radiator was chosen such that the heavier particle does not yet radiate, or is just below the threshold. The threshold for producing Cerenkov radiation is $\beta=1 / n$ and

$$
n^{2}=\frac{\gamma_{t}^{2}}{\gamma_{t}^{2}-1}
$$

where $\gamma_{t}$ is the relativistic factor:

$$
\gamma_{t}=\sqrt{\frac{1}{1-\beta_{t}^{2}}}=1
$$


For a particle's velocity relative to $c, \beta=0.9$ the aerogel counter should (in the absence of knock-on electrons) never fire. The detected contamination for particles having $\beta$ below the threshold velocity comes from accidental background (PMT dark current) and Čerenkov radiation produced by energetic knock on electrons ( $\delta$ rays).

\subsubsection{Gas Čerenkov}

The Gas Cerenkov detector was installed in the electron arm with the purpose to provide a better separation between $e^{-}$and $\pi^{-}$. The gas is $\mathrm{CO}_{2}$ at normal atmospheric pressure. The index of refraction for $\mathrm{CO}_{2}$ at atmospheric pressure is $n=1.00041$. This refraction index corresponds to a threshold velocity (relative to $c$ ) for producing the Čerenkov radiation of $\beta_{T h r}=\frac{1}{n}=0.99959017$. This corresponds to a momentum threshold, $p_{T h r}=\frac{m_{0} \cdot c}{\sqrt{1-\beta^{2}}}$, of $4.8 \mathrm{GeV} / \mathrm{c}$ for producing Čerenkov radiation by $\pi$ in the Gas Cerenkov detector ( $m_{0}$ is the rest mass of the particle). The threshold momenta for producing Čerenkov radiation with electrons is $0.017 \mathrm{GeV} / \mathrm{c}$. This means that only the electrons are able to fire the Gas Cerenkov detector since, for all our kinematics, the lowest momenta in the electron arm was $1.741 \mathrm{GeV} / \mathrm{c}$ and the highest momenta was $3.18 \mathrm{GeV} / \mathrm{c}$.

The efficiency of the Gas Čerenkov detector for detecting electrons is explained in detail in Section 3.8. Fig. 18 shows the ADC sum distribution for the Gas Cerenkov detector, during the E98-108 experiment, after subtracting the pedestals and after gain correction. 


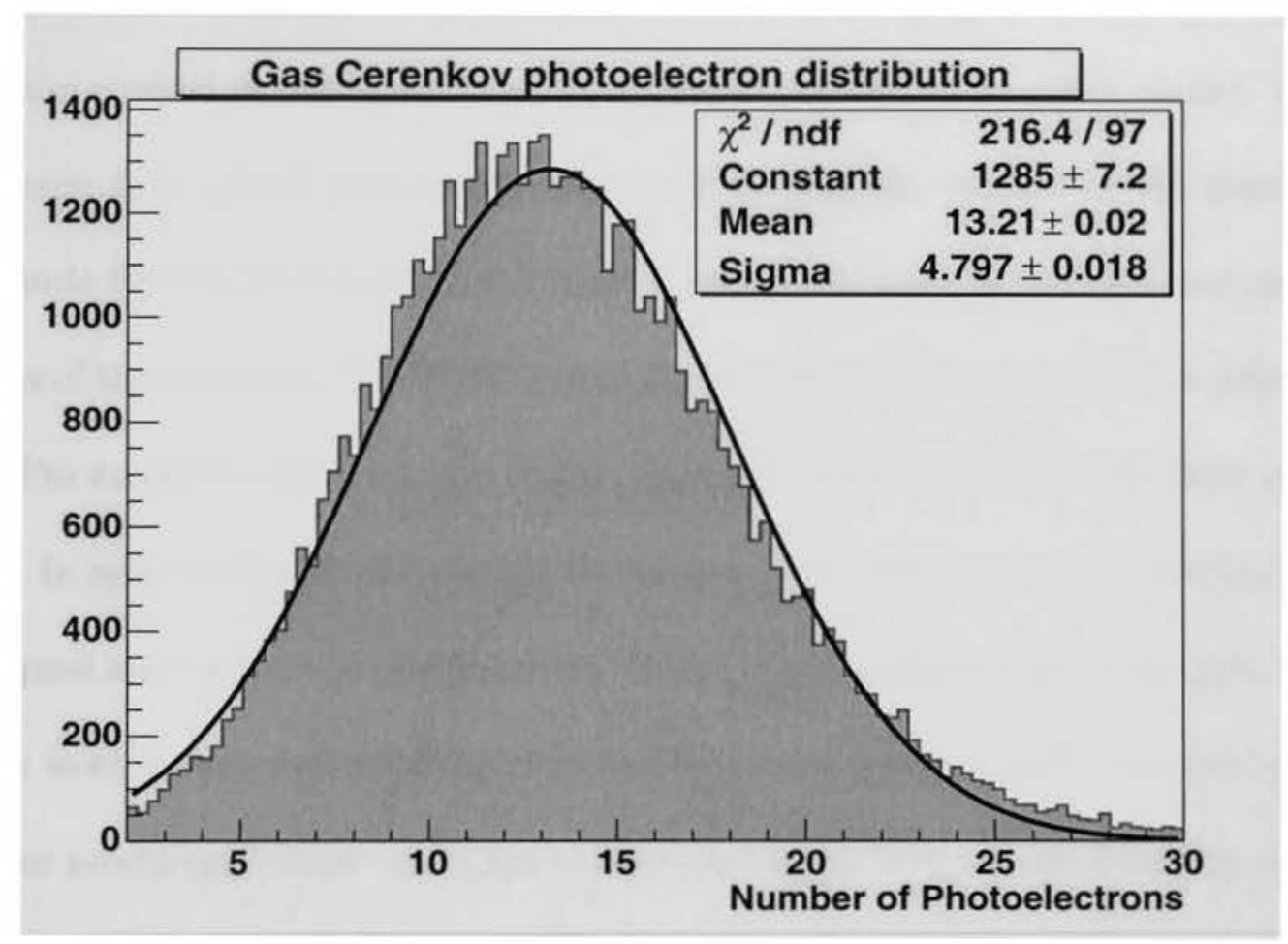

Figure 18: Gas Čerenkov ADC sum distribution fitted to a Gaussian.

\subsection{Aerogel Čerenkov detectors}

2.7.1 First Aerogel Čerenkov detector, $n=1.015$

The first aerogel Cerenkov detector with an refraction index of $n=1.015$ was built at Jefferson Lab by myself under the supervision of Bogdan Wojtsekhowski, JLab staff. The first diffusion box (see Fig. 19) consisted of an aluminum frame, a diffusion box, 24 Burle photomultipliers (whose quantum efficiency was previously determined), a honeycomb structure and the aerogel blocks (each block's dimensions were 
approximately $10 \times 10 \times 1 \mathrm{~cm}^{3}$ ). The aerogel blocks were supported by a honeycomb structure covered in two layers of millipore paper (backed-up by white paper). Thin aluminum foils, glued with epoxy, were used to seal the volume of the detector. The Burle PMT's, 8854 type, have a high quantum efficiency in both UV and visible regions of the spectrum. The PMT's were aligned on each lateral side of the diffusion box. The aerogel blocks were very fragile, especially those having the refractive index 1.015. In order to handle the aerogel blocks and align them inside the diffusion box, I designed and built an aerogel handling system based on aspiration techniques. The design worked very well allowing the handling and alignment of the aerogel blocks without producing "chips" and gaps in between blocks. The aerogel handling device allowed a tight packing of the aerogel blocks. The diffusion box was covered by two layers of reflective millipore paper $(0.22 \mu \mathrm{m}$ in thickness $)$ backed-up by a layer of white paper. The diffusion box was chosen as an alternative to the traditional light focusing design due to its highly reflective properties (greater than 95\%). The outer circular edges of the PMT's were also surrounded by millipore paper in order to reduce any black spots (where the light could have escaped without being recorded). The aerogel design was optimized such that the distance from the aerogel to the PMT's was minimized and the area covered by the PMTs was maximized. The PMT's maximum area criteria was based on the expected number of photoelectrons produced in the aerogel Cerenkov detector being proportional to the fraction of the total aerogel area covered by PMT's. The effective area of the aerogel that acted as a radiator was approximately $5440 \mathrm{~cm}^{2}$ for $A 1$. The thickness of the radiator was 9 


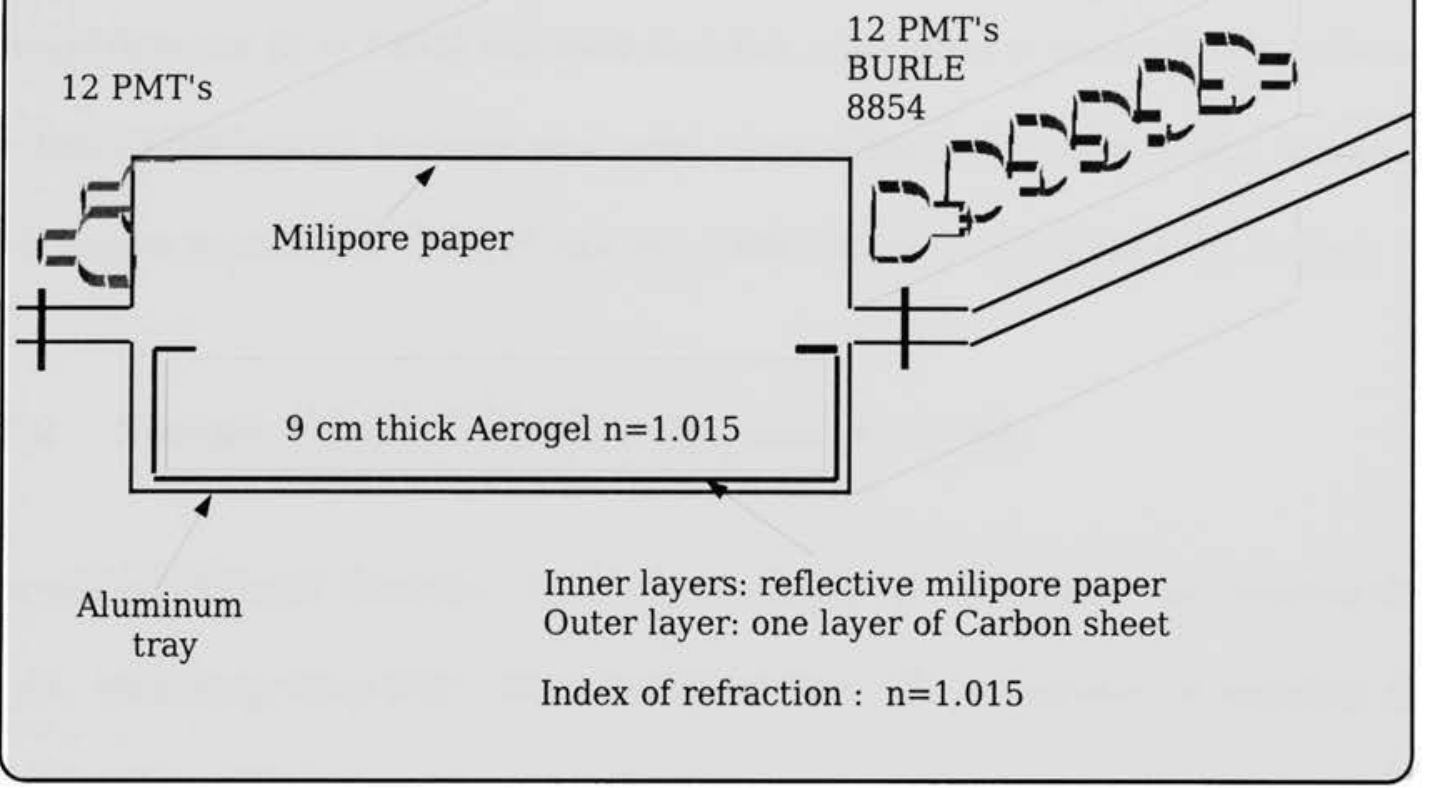

Figure 19: First diffusion box Aerogel Čerenkov detector.

$\mathrm{cm}$ (see Fig. 19). The refraction indices of the aerogel for both aerogel detectors were measured at JLab by Bogdan Wojtsekhowski (see reference [WOJ01]). The Aerogel Čerenkov detectors were placed in between the scintillator detectors in the hadron arm (the coincidence signal of the two scintillators was used to trigger the data acquisition system). This means a signal that fired the desired block in the aerogel was triggered by the two scintillators (in coincidence with each other) and also fired the wire chamber and therefore could be traced back to the target (as being a signal produced by a particle that originated from a reaction in the $\mathrm{LH}_{2}$ target). The kaon electroproduction experiment, E98-108, requires good particle identification 
and separation between kaons, $\pi^{+}$and protons. The kaons can be separated from $\pi$ in a range of momenta from $800 \mathrm{MeV} / \mathrm{c}$ to $2800 \mathrm{MeV} / \mathrm{c}$ (see Fig. 17). The first Aerogel detector $(n=1.015)$ was used to reject pions since it does not fire on kaons. We had to distinguish between two types of particles in the hadron arm, $\pi$ and $K$ with masses $m_{\pi}=139.57 \mathrm{MeV} / \mathrm{c}^{2}$ and $m_{K}=493.7 \mathrm{MeV} / \mathrm{c}^{2}$ (obviously $m_{\pi}<m_{K}$ ).

\subsubsection{Second Aerogel Čerenkov detector, $n=1.055$}

The second Aerogel Cerenkov detector was built by MIT after the construction of $A 1$, with help from FIU. They benefited from the experience of building the $A 1$ Čerenkov detector. The second Aerogel Črenkov detector, $n_{A 2}=1.055$ [ALC04], has a similar design to A1. The difference is that it uses 26 Photonis XP4572B/D1 photomultipliers which have a higher collection efficiency than Burle PMT's, although they are not as sensitive to UV radiation wavelengths. The radiator area for $A 2$ is about $30 \times 192 \mathrm{~cm}^{2}$ and the aerogel refractive index $n=1.055$. Another difference is the aerogel thickness of $A 2$ detector: $5 \mathrm{~cm}$ was chosen to maximize the number of $P E$ and minimize the number of scatterings until the light reaches the PMTs . The second Aerogel Čerenkov detector, $n=1.055$, does fire on kaons and $\pi$, the momenta threshold $p_{T h r}^{K}$ for kaons being $1550 \mathrm{MeV} / \mathrm{c}$, but not protons. The two Aerogel Čerenkov detectors were used together in order to distinguish $\pi^{+}$from $K^{+}$. The first Aerogel Cerenkov detector, $n=1.015$, does not fire on kaons up to a momenta of $2800 \mathrm{MeV} / \mathrm{c}$ and our highest momenta in the hadron arm was $2.542 \mathrm{MeV} / \mathrm{c}$, but does fire on pions. It means that $A 1$ can be used to reject the 


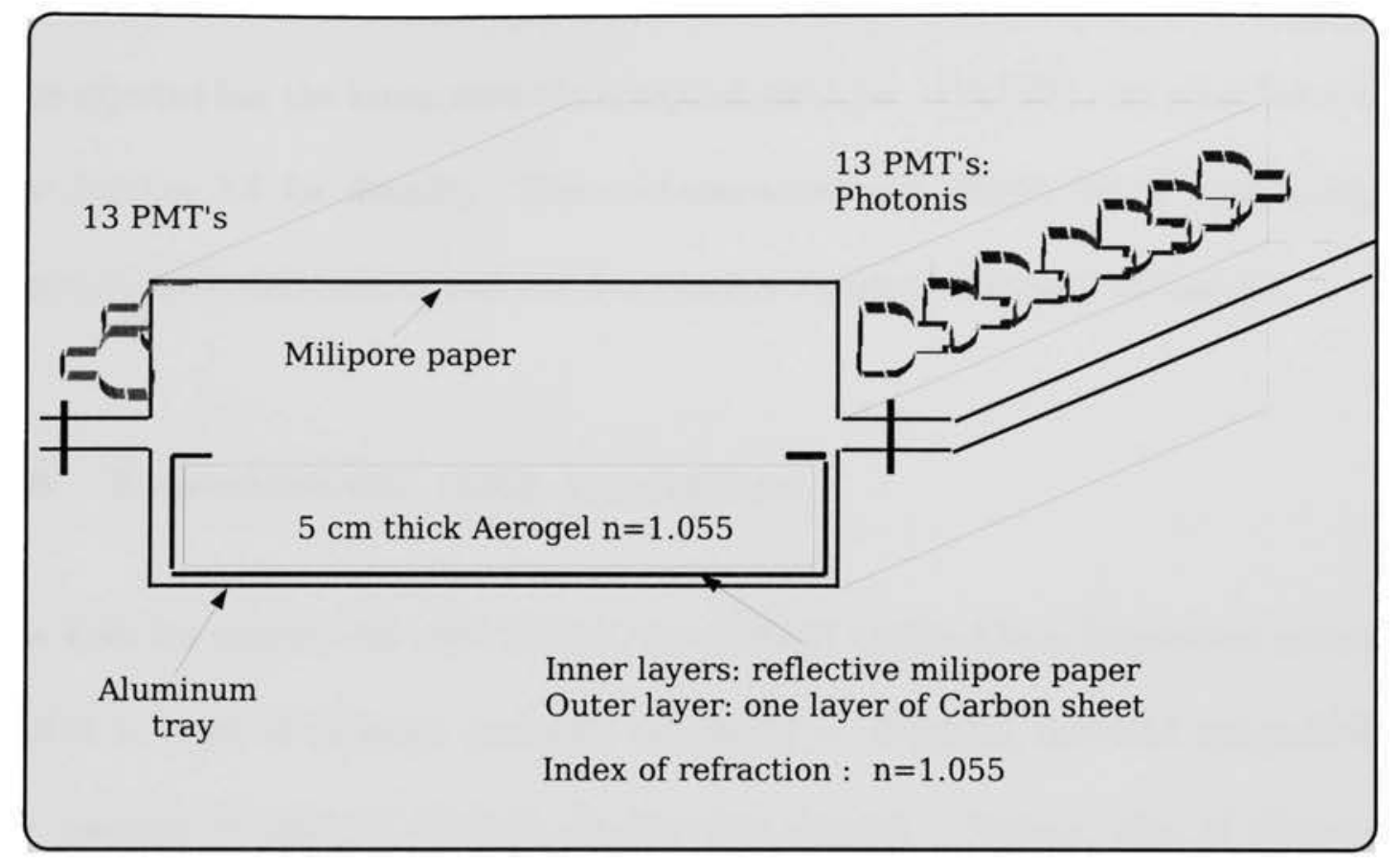

Figure 20: Second diffusion box-Aerogel Čerenkov detector.

pion background from the spectra by applying a software cut on the second aerogel detector $A 2(\mathrm{n}=1.055)$.

\begin{tabular}{|c|c|c|c|}
\hline Refraction index n & $\pi_{T h r}(\mathrm{MeV} / \mathrm{c})$ & $K_{T h r}(\mathrm{MeV} / \mathrm{c})$ & $p_{T h r}(\mathrm{MeV} / \mathrm{c})$ \\
\hline 1.015 & 814.84 & 2882.21 & 5477.87 \\
\hline 1.055 & 437.98 & 1549.20 & 2944.38 \\
\hline
\end{tabular}

Table 2: Table with particles' threshold momenta for different aerogel refractive indexes. 
A combination of software cuts between $A 1$ and $A 2$ was used such that the pions were rejected but the kaons were the accepted particles: $A 1 \& \overline{(A 2)}-A 1$ And Not $(A 2)$ (see Section 3.6 for details). The contamination and efficiencies of both aerogel detectors were determined and are described in detail in Sections 3.6 and 3.7.

\subsection{Experimental data acquisition}

The E98-108 experiment used CODA, the CEBAF Online Data Acquisition system. CODA is a set of software packages developed at Jefferson Lab and designed for the readout of data in nuclear physics experiments. Various bits of data are recorded by the software starting with the information about the event, the run number, information from each of the detectors in both arms (electron and hadron), scaler data, spectrometer magnet settings, angles of the scattered particles, target temperature and pressure, spectrometer position, beam characteristics (energy, position, size, current) and associated scalers used to calculate, e.g., deadtimes. The CEBAF Online Data Acquisition system uses hardware Read Out Controllers (ROC's) which constitute the interface between the detectors and the recording computer, an event builder which continues the information coming from the ROC's and incorporates all header information, and an online analyzer and data distributing system whose purpose is to analyze and write the physics data to the hard drive where it is eventually transferred to tapes. An online analyzer package is used to analyze data while acquiring it and to diagnose faulty equipment. The beginning of the data 
file contains a header with the status of the run and a map containing the detector configuration.

\subsubsection{Data acquisition system}

The data acquisition system for each separate spectrometer consists of VME systems, one trigger supervisor, at least one fastbus electronic crate, time to digital converters, and analog to digital converters. The data acquisition system reads the values of the scalers every 4 seconds. The events are grouped together based on their type (singles or coincidences). The main trigger on Electron/Hadron arm, $T 1 / T 3$, is formed by overlapping the signals from the two scintillators $S 1$ and $S 2(S 3$ and $S 4$ for the Hadron arm). The overlap is performed by sending the scintillators' signals to a logical $A N D$ unit. In order to consider that a paddle had fired, both PMT's attached at the ends of the paddle were required to fire.

\subsubsection{Trigger electronics; block diagram}

The single arm trigger for data acquisition in Hall $\mathrm{A}$ is schematically displayed in Fig. 21. The memory lookup units (MLU in the Fig. 21 and 22) in each spectrometer arm generated a logical output (S-ray) when both PMT's of a scintillator paddle (in the first scintillator plane S1) fired and both PMT's (in the second scintillator plane $S 2$ ) of an adjacent or coincident paddle fired. The coincidence trigger, T5, for E98108 experiment is formed by sending the single arm S-ray triggers (described above) 
Single Arm Triggers in Electron/Hadron Spectrometer

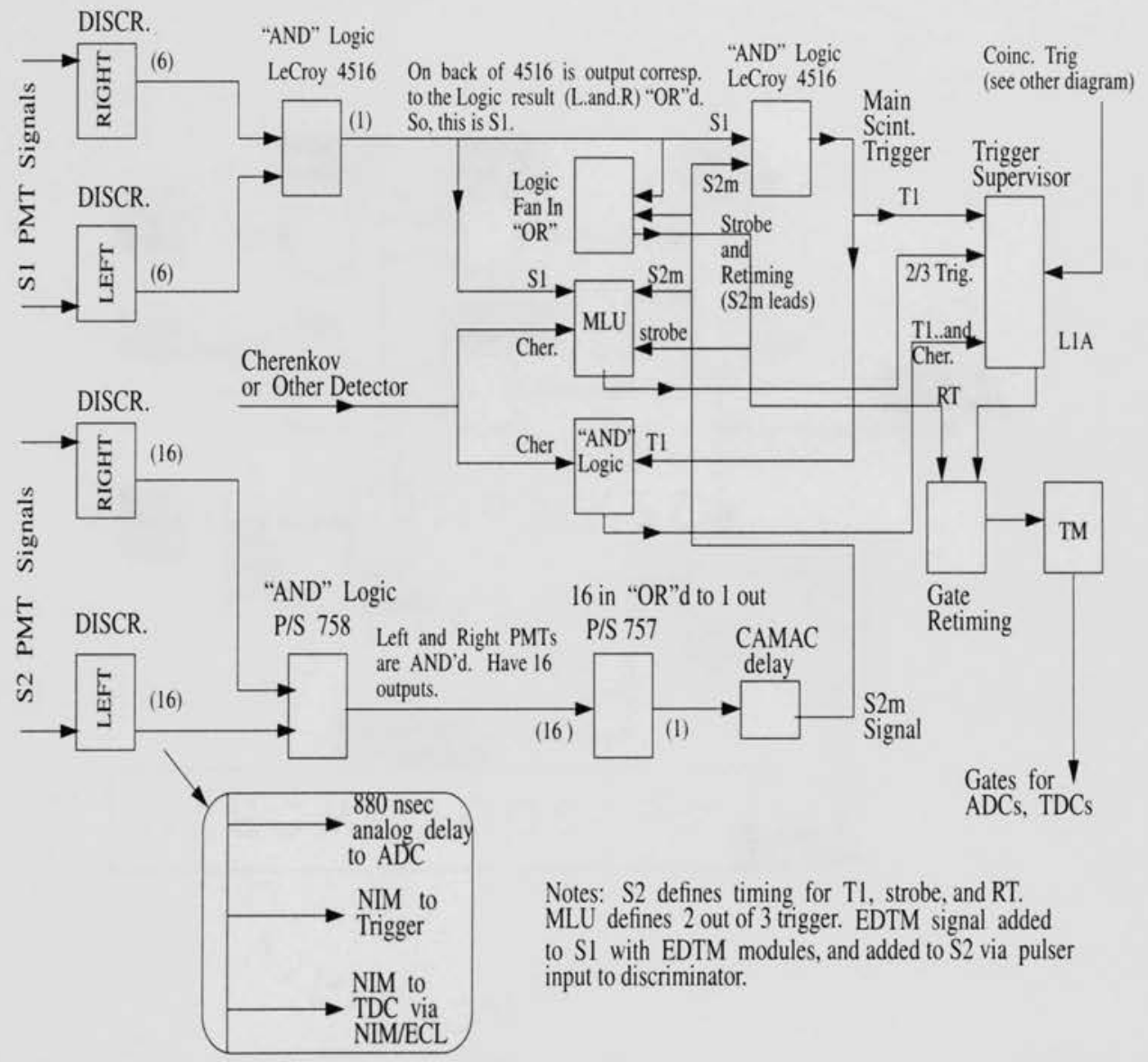

Figure 21: Single arm trigger for E98-108; modified figure taken from [MIC02].

to a logical $A N D$ unit. There is a time window setup of approximately $100 \mathrm{~ns}$ for the coincidence to be accepted as such. The coincidence trigger for data acquisition in Hall A at Jefferson Lab as used in E98-108 experiment is schematically displayed in Fig. 22. 


\section{Coincidence Trigger}

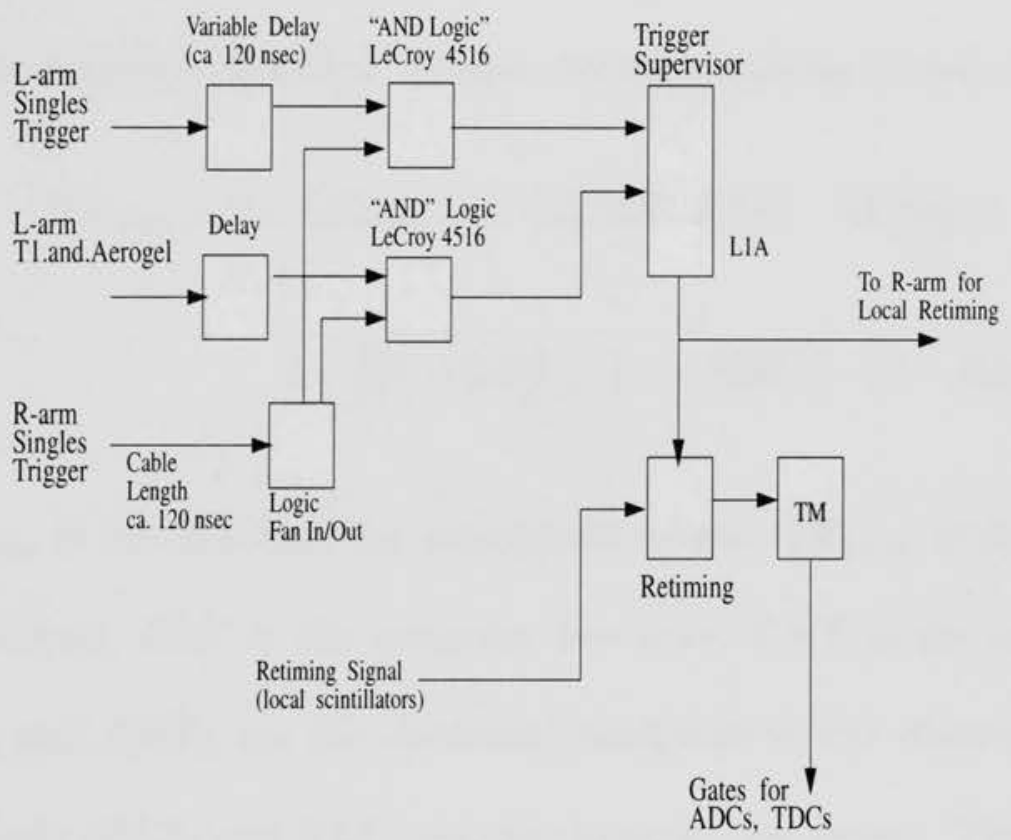

Figure 22: Coincidence trigger for E98-108; modified figure taken from [MIC02].

During the E98-108 experiment all triggers were prescaled except for the coincidence trigger, $T 5$, since we were interested in $(e, K)$ coincidences. 


\subsubsection{Electronic and computer deadtime determination}

To determine the electronic deadtime, we started running without the memory lookup unit [MIC01]. The trigger was formed by an overlap (logical $A N D$ ) of the two scintillator planes S1 and S2. In this way the electronic deadtime was reduced. To determine the deadtime (see Figs. 23 and 24) the following formula was used:

$$
\begin{aligned}
D T_{\text {Coinc }}=1-L T_{\text {Coinc }}=1-\left(C L T \cdot E L T_{L} \cdot E L T_{R}\right)= \\
1-\left[(1-C D T) \cdot\left(1-E D T_{L}\right) \cdot\left(1-E D T_{R}\right)\right]
\end{aligned}
$$

where $D T_{\text {Coinc }}$ is the deadtime for coincidence events, $L T_{\text {Coinc }}$ is the livetime for coincidence events, $C L T$ is the computer live time, $C D T$ is the computer dead time, $E D T_{L}$ and $E D T_{R}$ are the electronic deadtimes in the electron and hadron arm respectively ( $E L T_{L}$ and $E L T_{R}$ are the electronic live times). The fortran code, developed by Bodo Reitz [REB03], to calculate the deadtime was also used in other experiments. The code is based on the ratio of triggers to accepted triggers measured using the scalers from the $T D C$ s and from the calibrating pulsers. 


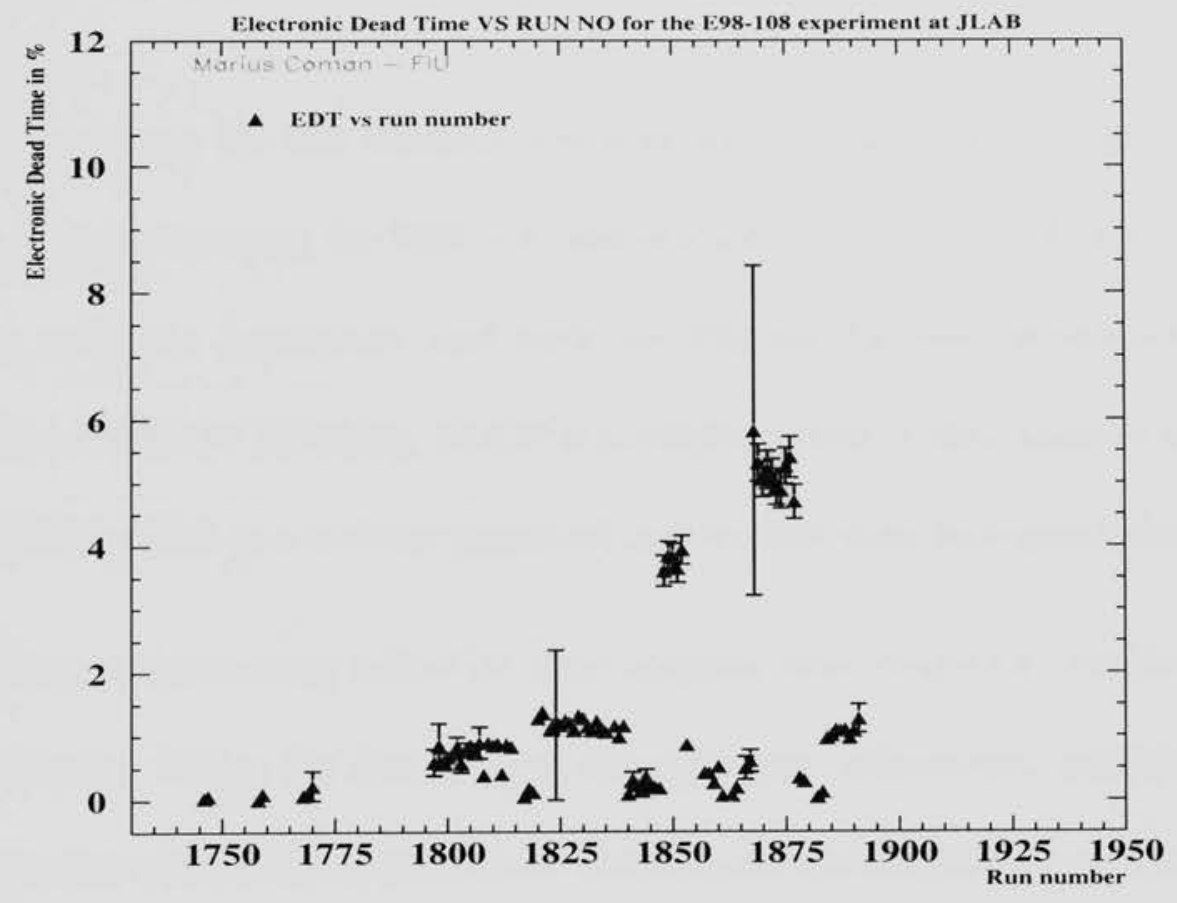

Figure 23: Electronic deadtime vs run number, 1st set of runs.

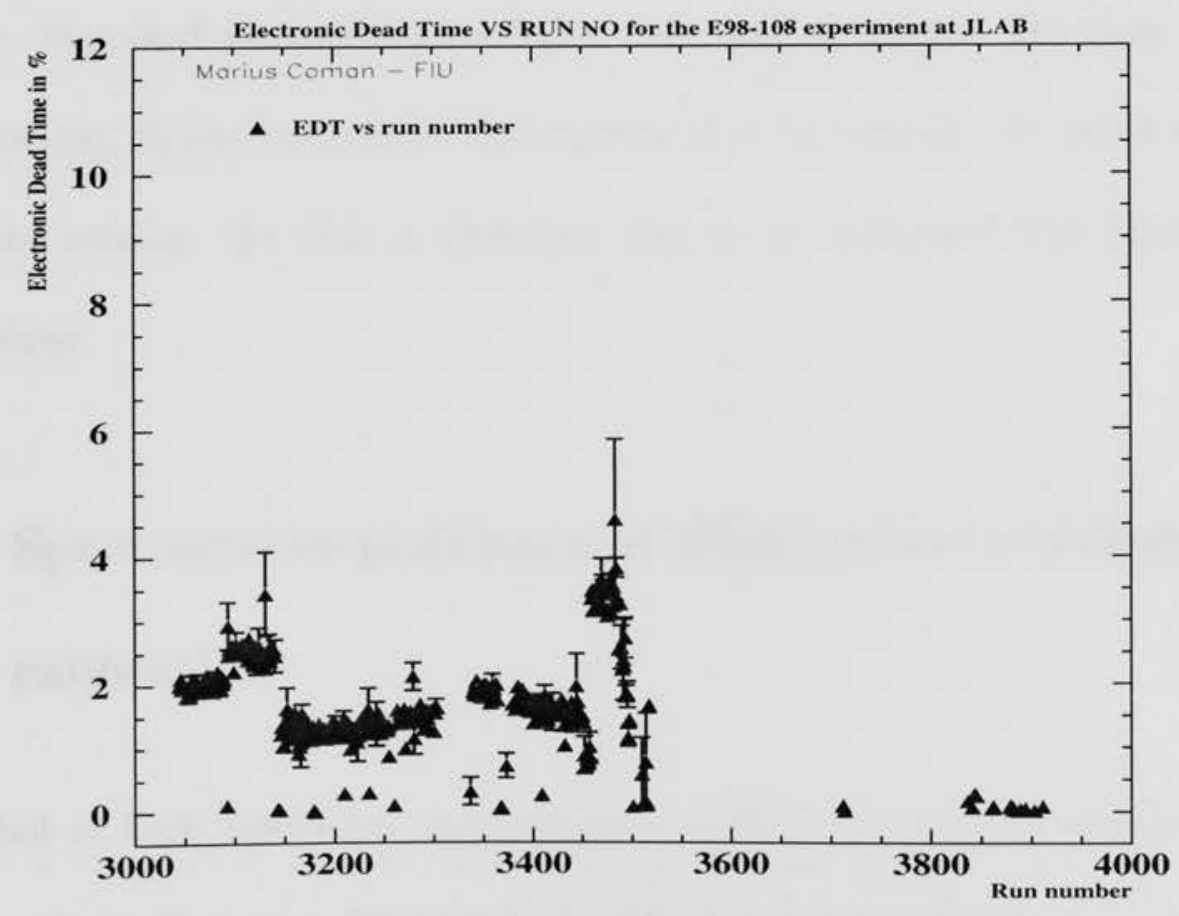

Figure 24: Electronic deadtime vs run number, 2nd set of runs. 


\section{Calibration and efficiency measurements}

The data analysis for this research was conducted by using the computer package

Espace (Event Scanning for Hall A Collaboration Experiments) [ESP02] for online analysis and data acquisition, and both the Physics Analysis Workstation (PAW) [PAW01] and ROOT [ROO03]. ROOT is an object oriented data analysis framework, from CERN, which is a tool for graphical display and data histogramming.

Various programming codes for data analysis were created to ensure a reliable data analysis. Codes for determining the detectors' efficiencies, particle tracking, reconstruction and identification were created and developed and are presented in the following sections. The detection efficiencies were optimized and performed run by run. Standard statistical procedures were used to analyze the data: frequency distributions, fitting procedures and statistical error analysis. In order to measure the cross section, the Hall A detectors had to be calibrated and their efficiency determined.

\subsection{Spectrometer position and offsets determination; Optics calibration}

The Hall A high resolution spectrometers consist of a QQDQ system with the quadrupoles acting as a focusing lens. The focusing properties in the dispersive direction are point to point. The coordinates of the interaction vertex at the target, 
$y_{t g t}, \delta, \theta_{t g t}$ and $\phi_{t g t}$ are determined from the coordinates at the focal plane, $x_{f p}$, $y_{f p}, \theta_{f p}$ and $\phi_{f p}$ [ESP02]. This reconstruction of the coordinates at the target is done using the optics tensor. The coordinate transformation is essential in determining the spectrometers' acceptance and the coordinates relationships used for the transformation can be found in Ref.[ALC04]. The calculation of the optimal

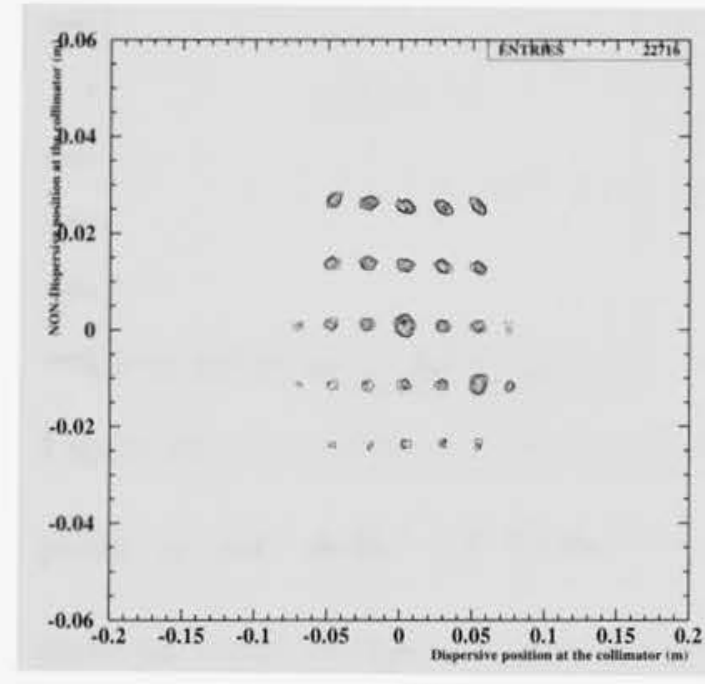

Figure 25: Sieve slit ${ }^{12} \mathrm{C}$ target, Elec-

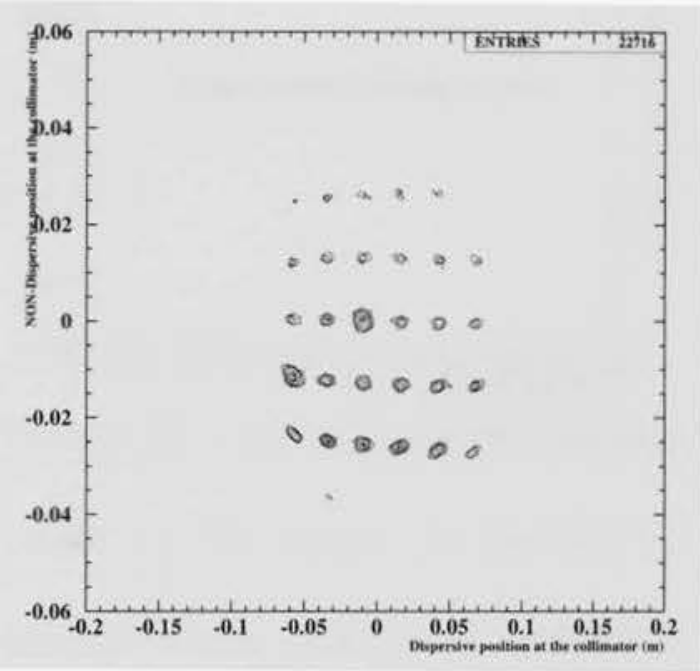

Figure 26: Sieve slit ${ }^{12} \mathrm{C}$ target, Hadron Arm: Non-dispersive position at the collimator (in $m$ ) versus dispersive position at the collimator (in $m$ ).

matrix elements is done using the target coordinates determined from surveys and the focal plane coordinates as reconstructed by ESPACE's tracking routine [ESP02]. The matrix elements were optimized by Nilanga Liyanage [LIY02] for the experiment 
E94-104 that ran before and during E98-108. The calibration was accomplished using the sieve slit (see Fig. 25 and Fig. 26) and the ${ }^{12} \mathrm{C}$ targets (see Fig. 27 and Fig. 28). From the figures mentioned above, it can be seen that the reconstruction is not as

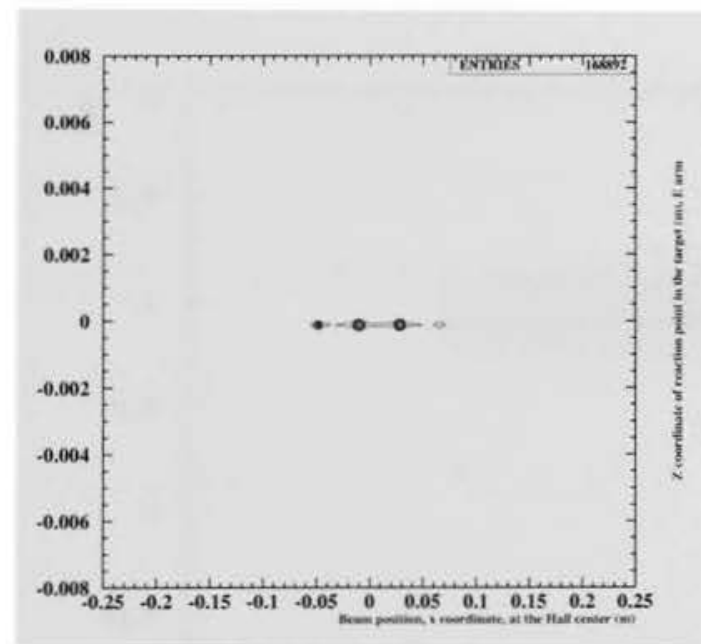

Figure 27: $z$ coordinate of the reaction point in the target (in meters) vs rastered beam position ( $\mathrm{x}$ coordinate at the Hall A center in meters), E arm.

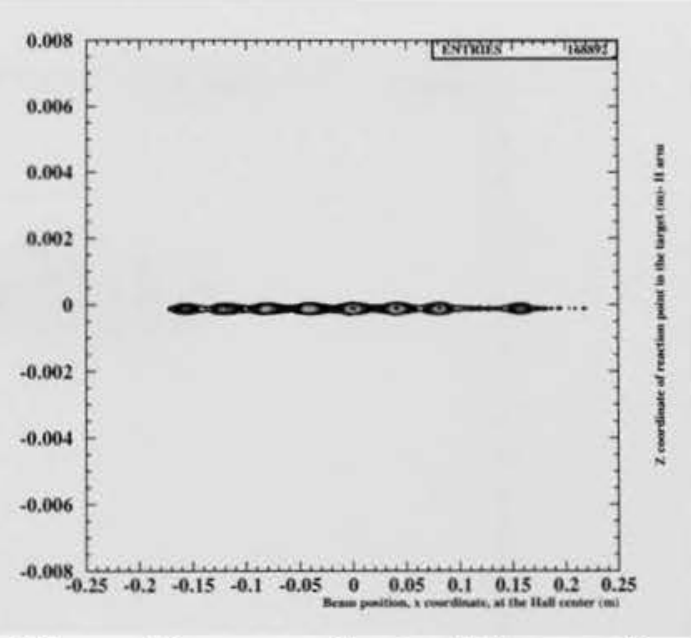

Figure 28: $z$ coordinate of the reaction point in the target (in meters) vs rastered beam position ( $\mathrm{x}$ coordinate at the Hall A center in meters), $\mathrm{H}$ arm.

good at the edge of the acceptance as it is in the center. This was taken care of by excluding the events at the edge of acceptance using the r-function cut as described in section 4.3. The software's angular accuracy was determined by comparing the holes' angles from surveys [JEF03] to angles from analyzing data. The position of the sieve slit was extracted from the data by fitting the angles $\phi$ at the target with cuts on $\theta$ at the target $\left(\phi_{\text {Target }}\right.$ with cuts on $\left.\theta_{\text {Target }}\right)$ respectively fitting $\theta$ at the target with cuts on $\phi_{\text {Target }}($ see appendix G). 
The optimization of the particles' reconstructed velocity relative to the speed of light was done using the routines from Nilanga Liyanage [LIY02] and the result after the optimization is shown in Fig. 29 for electrons travelling at $\beta=1$.

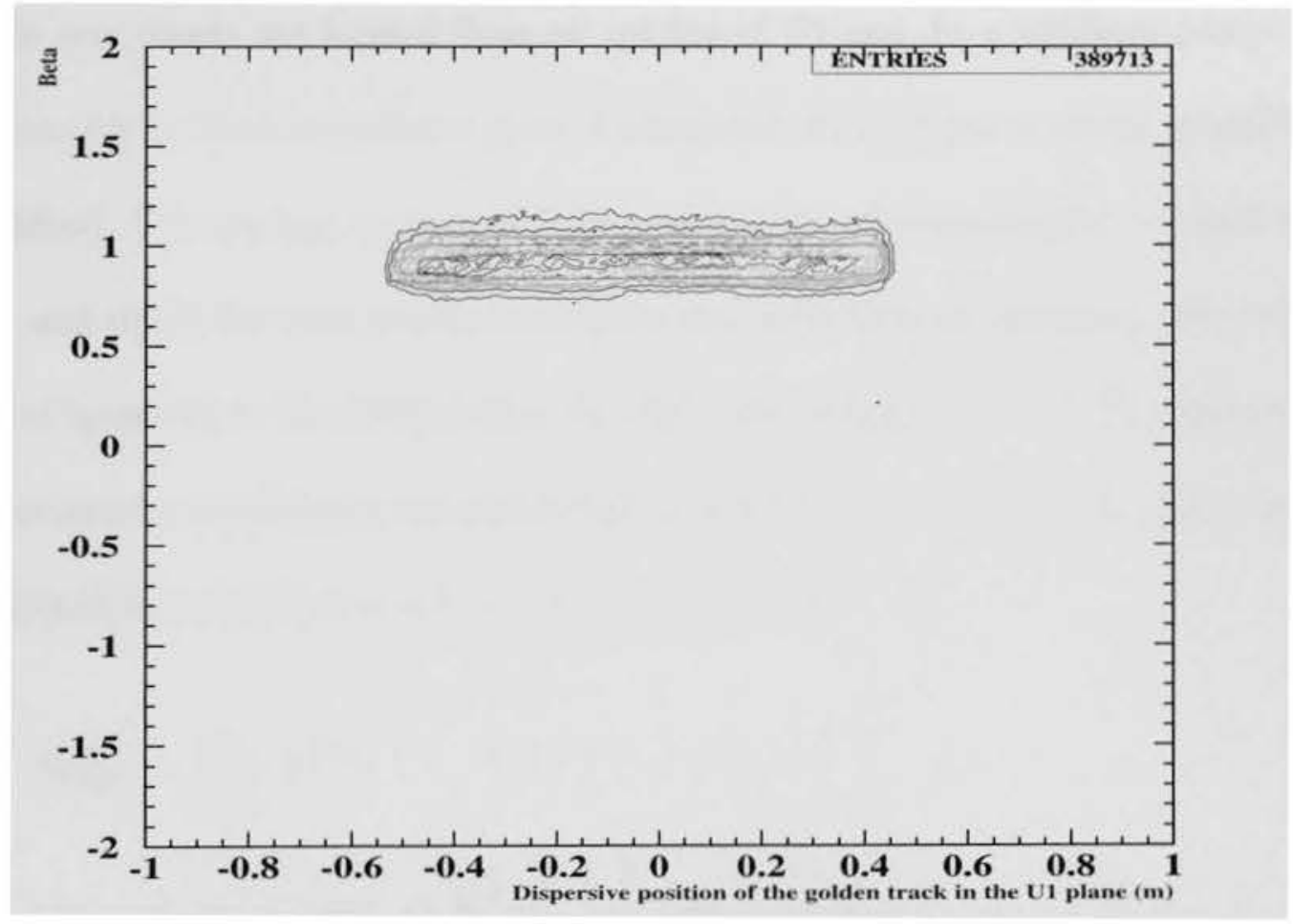

Figure 29: Beta optimization, Left Arm for electrons. 


\subsection{Scintillators' efficiencies}

We determined the scintillator efficiencies for the experiment by using the prescaled trigger types $1-5$. The main triggers ( $T 1$ on electron arm and $T 3$ on hadron arm) for single arm events are formed from an overlap of $S 1$ and $S 2$ scintillator planes (see Section 2.8.2). Each scintillator plane is composed of six identical plastic scintillators (paddles). The overlap consists of $A N D$-ing $(\wedge)$ the photomultipliers on both sides (left and right) for each paddle scintillator and then $O R$-ing (applying the $O R-\vee$ logical operator) to the paddles thus obtained. We calculated the overall scintillators' efficiencies by multiplying the individual planes efficiencies in each arm since we are interested in coincidence events for the entire analysis:

$$
\epsilon_{\text {Total }}^{\text {Scin }}=\prod_{k=E, H} \epsilon_{k}^{\text {Scin }}
$$

where the overall scintillator efficiency per arm, $\epsilon_{H}$ and $\epsilon_{E}$ were calculated by:

$$
\epsilon_{E}^{S c i n}=\frac{G O O D_{T_{1}}+G O O D_{T_{5}}}{G O O D_{T_{1}}+G O O D_{T_{5}}+G O O D_{T_{2}}}
$$

and

$$
\epsilon_{H}^{S c i n}=\frac{G O O D_{T_{3}}+G O O D_{T_{5}}}{G O O D_{T_{3}}+G O O D_{T_{5}}+G O O D_{T_{4}}}
$$

being the scintillator efficiencies for each arm (here $G O O D_{T_{i}}$ represent the good trigger types, main triggers $T_{1}$ and $T_{3}$ and loose triggers $T_{2}$ and $T_{4}$ ). The formula we used to determine the good trigger types for each arm are:

$$
G O O D_{T_{k}}=\left[\left(T_{k} \cdot P S_{k}\right) \bigwedge S 1 \bigwedge S 2 \bigwedge G O O D_{V D C} \bigwedge \delta p \bigwedge \check{C} \text { erenkov }\right]
$$




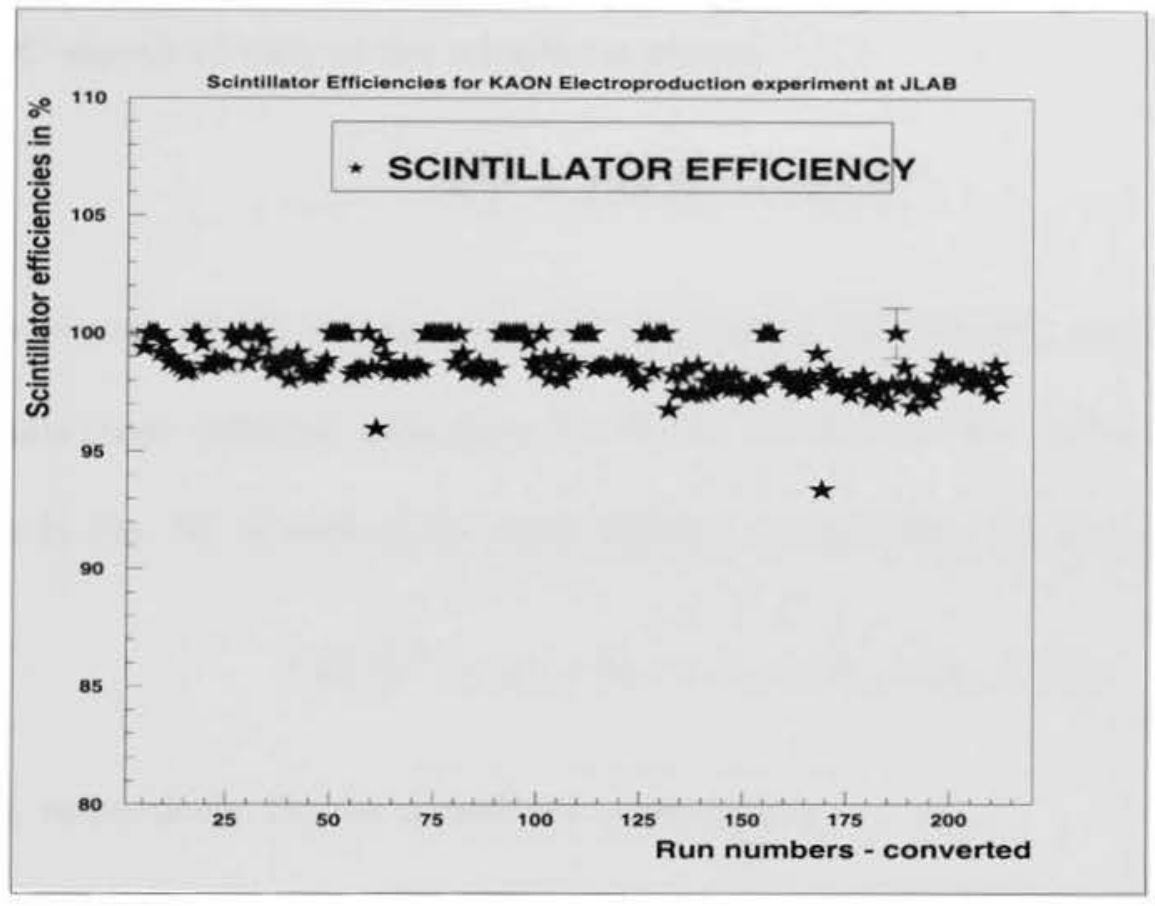

Figure 30: Total Scintillator efficiencies.

with the $i$-th index representing the main triggers $(1,3)$ or the loose triggers $(2,4)$, $S_{1}, S_{2}$ being a good signal in the scintillator planes, $P S_{k}$, being the prescale factors, $\delta p$ the relative momenta cut, and Čerenkov the standard cut applied on the Čerenkov detector (for the scintillator efficiency in the electron arm the Cerenkov cut refers to the Gas Črenkov detector whereas for the the scintillator efficiency in the hadron arm it refers to the second aerogel Čerenkov detector, $A 2$, cut for pions). We used the same cuts for the aerogel detectors in the hadron (and Gas Cerenkov in the electron) arm as those we used for determining the yield from missing mass spectra. Also the relative momentum cut, $\delta p= \pm 4 \%$, was the standard cut used in the analysis. A 
good signal in the scintillators' planes consisted of a logical $A N D$ between the overall $T D C$ signals of each of the scintillator planes:

$$
S_{1,2}^{E, H}=T D C_{S 1}^{E, H} \wedge T D C_{S 2}^{E, H}
$$

The overall TDC's signals consisted of a logical $O R$ between the individual TDC signals (that collected data from the PMTs located at both ends of the paddles as seen in Fig. 16) of each of the six scintillators' constituent paddles:

$$
T D C_{S 1}^{E, H}=S_{1,1} \vee S_{1,2} \vee S_{1,3} \vee S_{1,4} \vee S_{1,5} \vee S_{1,6}
$$

and, respectively, for the second scintillator plane

$$
T D C_{S 2}^{E, H}=S_{2,1} \vee S_{2,2} \vee S_{2,3} \vee S_{2,4} \vee S_{2,5} \vee S_{2,6}
$$

where the first subscript index refers to the scintillator plane and the second index refers to the paddle number. This means at least one paddle was required to fire in order to consider it a recordable event (together of course with fulfilling the required cuts). And finally $S_{i, j}$ is a logical $A N D$ between the left and right side TDCs:

$$
S_{i, j}=T D C_{i, j}^{L e f t} \wedge T D C_{i, j}^{R i g h t}
$$

for each of the $j=1,6$ scintillator planes. The same formulas apply for both electron and hadron arms. We defined a good signal in the vertical drift chambers as being the logical $A N D$ operator, between one single track and the events that fired all four $V D C$ planes (the multiplicity-number of hits- as established in 3.5) :

$$
\text { Mult }_{i} \wedge \text { Mult }_{j} \wedge \text { Mult }_{k} \wedge \text { Mult }, \wedge 1 \_ \text {Track }
$$


for each of the $V D C$ planes in both arms (electron and hadron). The results for the scintillator efficiencies are displayed in graphs 30 to 34 . For all the graphs ( 30 to 34 )

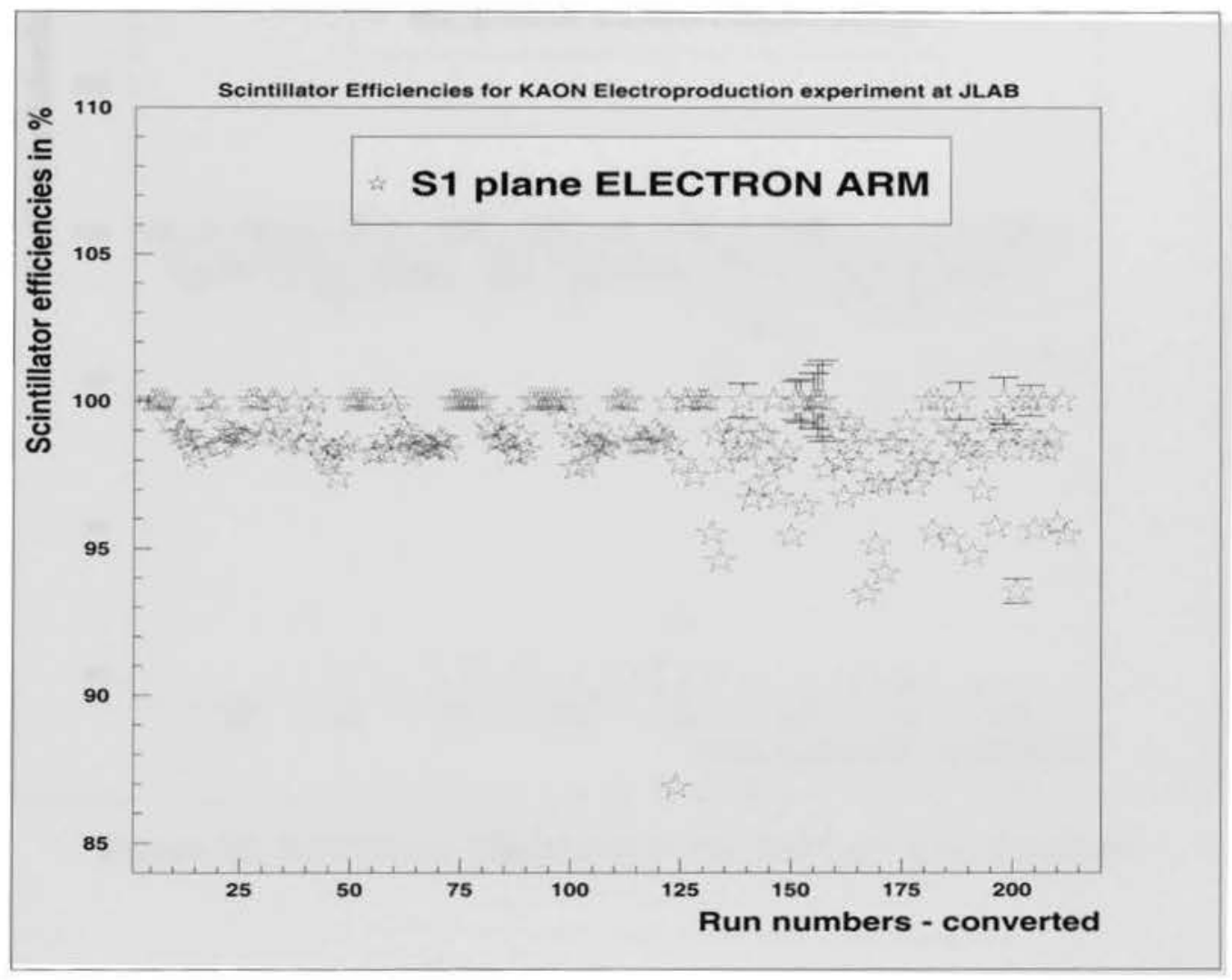

Figure 31: Scintillator efficiencies in the Electron arm, $S 1$ plane.

representing the scintillator efficiencies most of the errors are hidden by the marker's size. The errors are all less than $0.4 \%$. The run numbers have been converted (1 corresponding to the first run number for the experiment, 1735) to ensure clarity. There was a gap between the run numbers due to the fact that we acquired data in both 2001 and 2002. From figures 30 and 34 it looks as if there are two trends of the efficiencies that are not statistical fluctuations. We have also determined the scintillator efficiencies for different particle types in the hadron arm, calculating the 


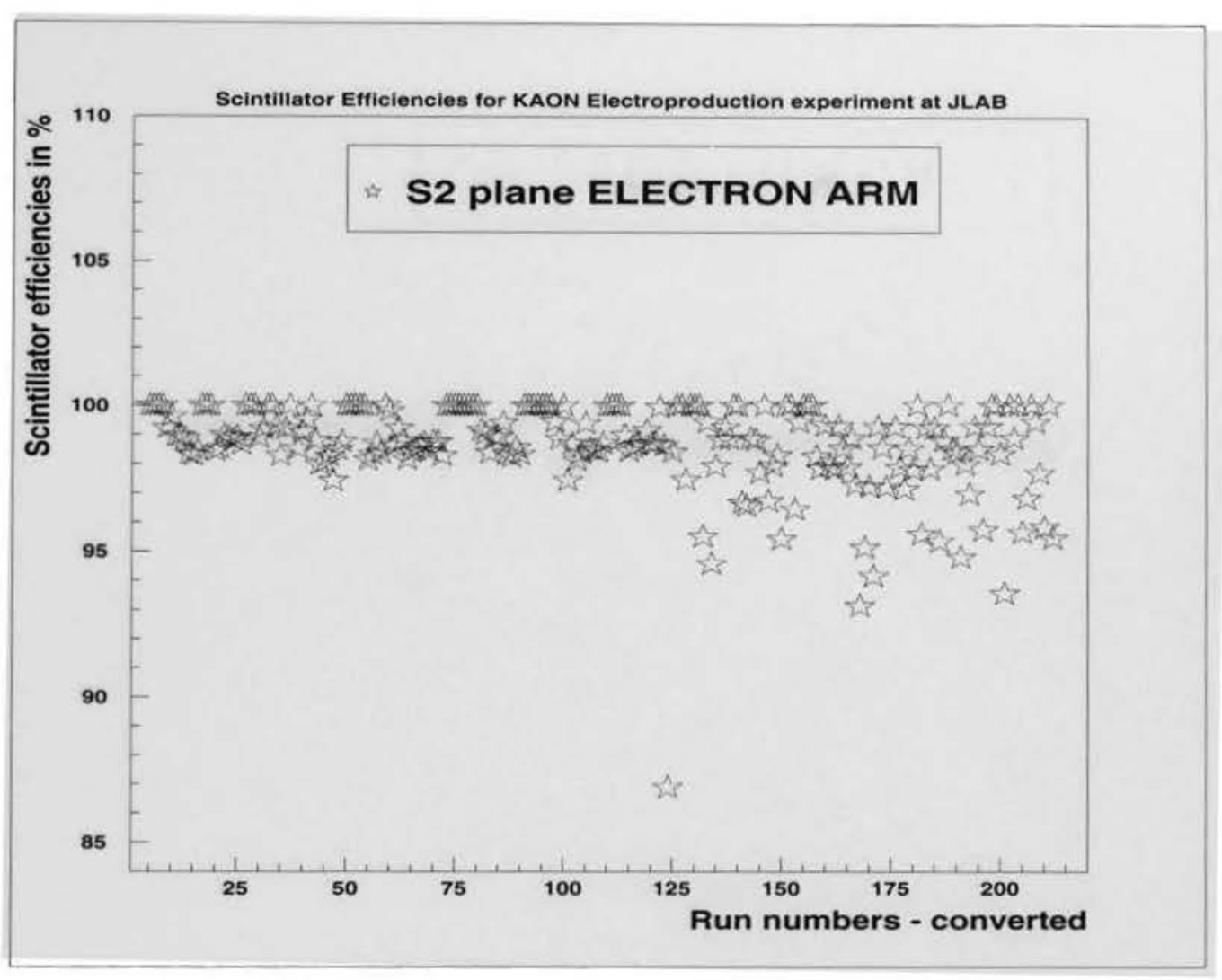

Figure 32: Scintillator efficiencies in the Electron arm, $S 2$ plane.

efficiencies for both protons and pions (see Fig. 35 for the first scintillator planes and Fig. 36 for second scintillator planes). The standard Gas Čerenkov cut, used in E98-108 analysis, was between $A D C$ channel 150 and channel 5000 (see section 3.8). The results show that the difference between scintillator efficiencies in the hadron arm for different types of particles (protons and pions) ranges between $0.89 \%$ to $1.33 \%$ for the first scintillator plane, $S_{1}$, and between $0.061 \%$ and $0.18 \%$ for the second scintillator plane, $S_{2}$ and the differences are within the error with which the efficiency was determined. The sensitivity of the scintillator efficiency to the VDC 


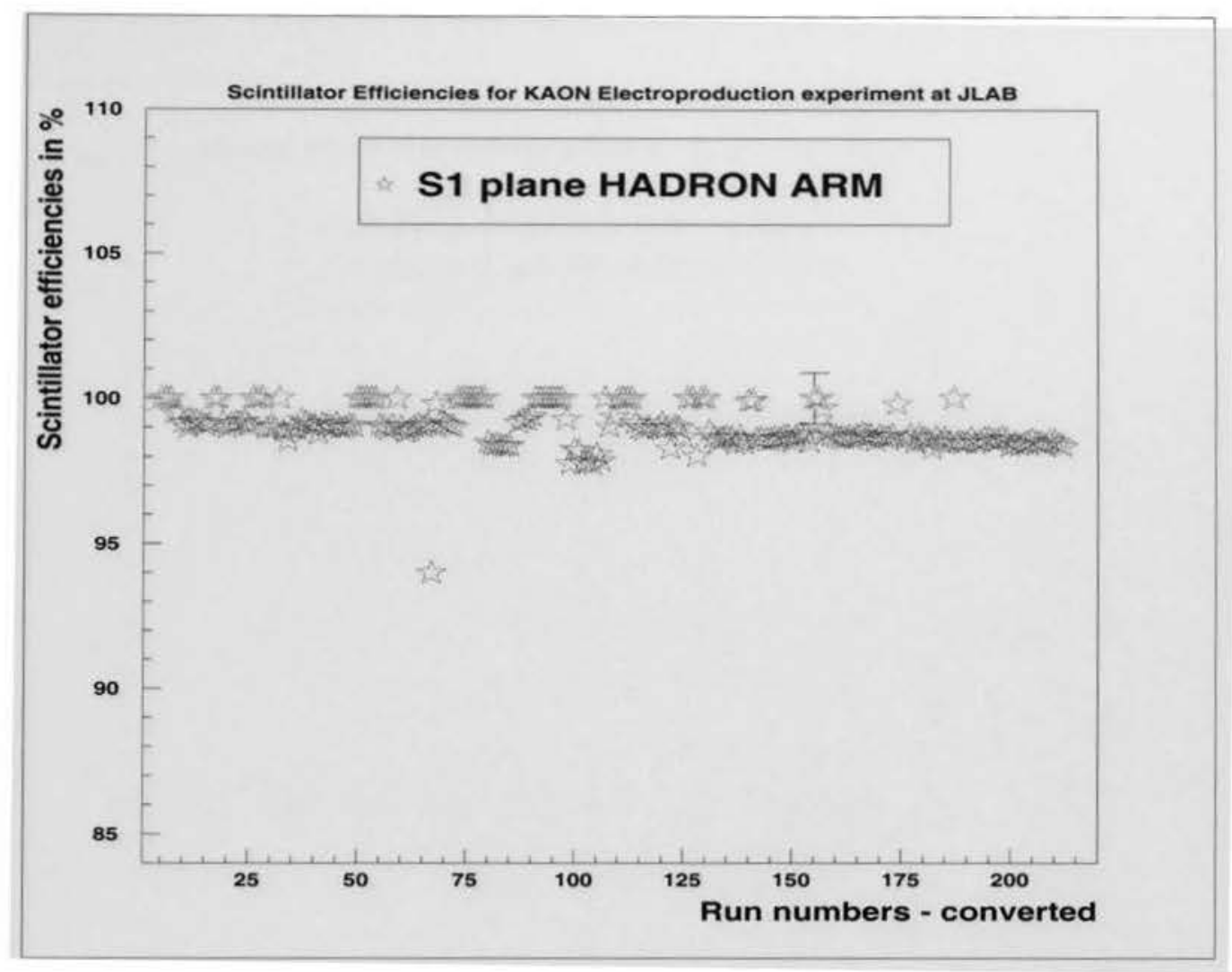

Figure 33: Scintillator efficiencies in the Hadron arm, $S 1$ plane.

cut was also determined using the same formula as Eq. 16 but where the $V D C$ cut has been removed.

$$
G O O D_{T_{k}}=\left[\left(T_{k} \cdot P S_{k}\right) \bigwedge S 1 \bigwedge S 2 \bigwedge \delta p \bigwedge \text { Cerenkov }\right]
$$

The sensitivity of the scintillator efficiencies (determined using Equation 17) to the $V D C$ cuts, the relative momenta cuts and the Čerenkov detectors cuts (aerogels in the hadron arm and gas Čerenkov in the electron arm) together with the relative errors is presented in details in Appendix K. Successively excluding the individual cuts (one at a time), we calculated the scintillators efficiency for both planes in each 


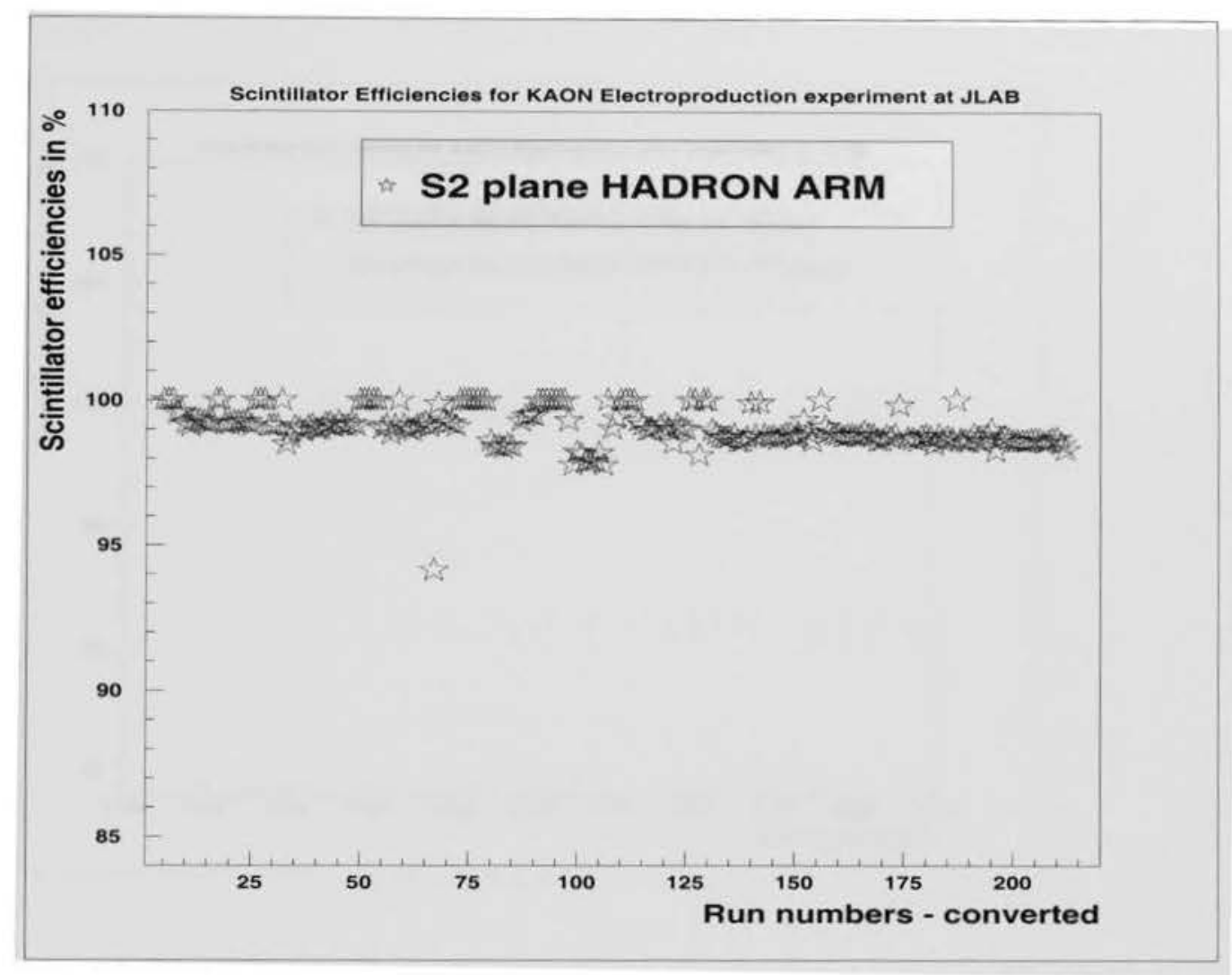

Figure 34: Scintillator efficiencies in the Hadron arm, $S 2$ plane.

arm and compared them with the efficiencies calculated when all cuts were present. The sensitivity to the $V D C$ cut ranges from $0.12 \%$ to $1.22 \%$ for the $S_{1}$ scintillator in the hadron arm, and from $0.10 \%$ to $1.26 \%$ for the $S_{2}$ scintillator. The sensitivity to the Gas Cerenkov cut ranges from $0.3 \%$ to $2.63 \%$ for the first scintillator plane, $S_{1}$ and from $0.2 \%$ to $2.76 \%$ for the second scintillator plane, $S_{2}$. The sensitivity to the Gas Cerenkov in the $E$ arm was determined knowing that the solid angle for the Gas Cerenkov is different than the solid angle subtended by the surface of the $V D C^{\prime} s$. 


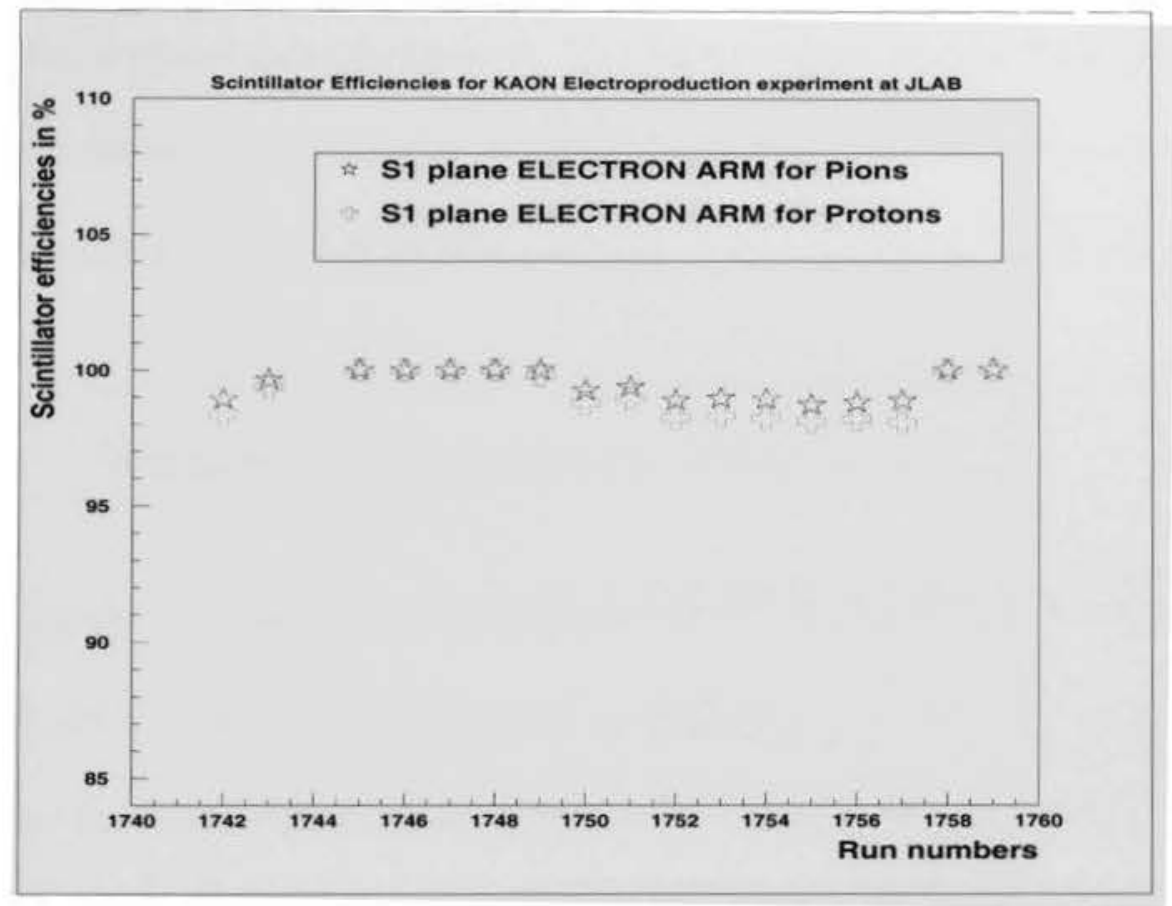

Figure 35: Scintillator efficiencies in the electron arm ( $S 1$ plane) for different types of particles - protons and pions.

\subsection{Boiling corrections}

The corrections for the variations of the $\mathrm{LH}_{2}$ target density were partially based on previous studies done with the $\mathrm{LH}_{2}$ beer can [ARM03]. The density correction are listed for each kinematic in Tables 10 to 13 in Appendix A. The systematic error in the target's density (see Table 9) was calculated by varying (in the Monte Carlo simulation code) the value of the density with $\pm 2 \%$ from the nominal value and then determining the change in the cross section. 


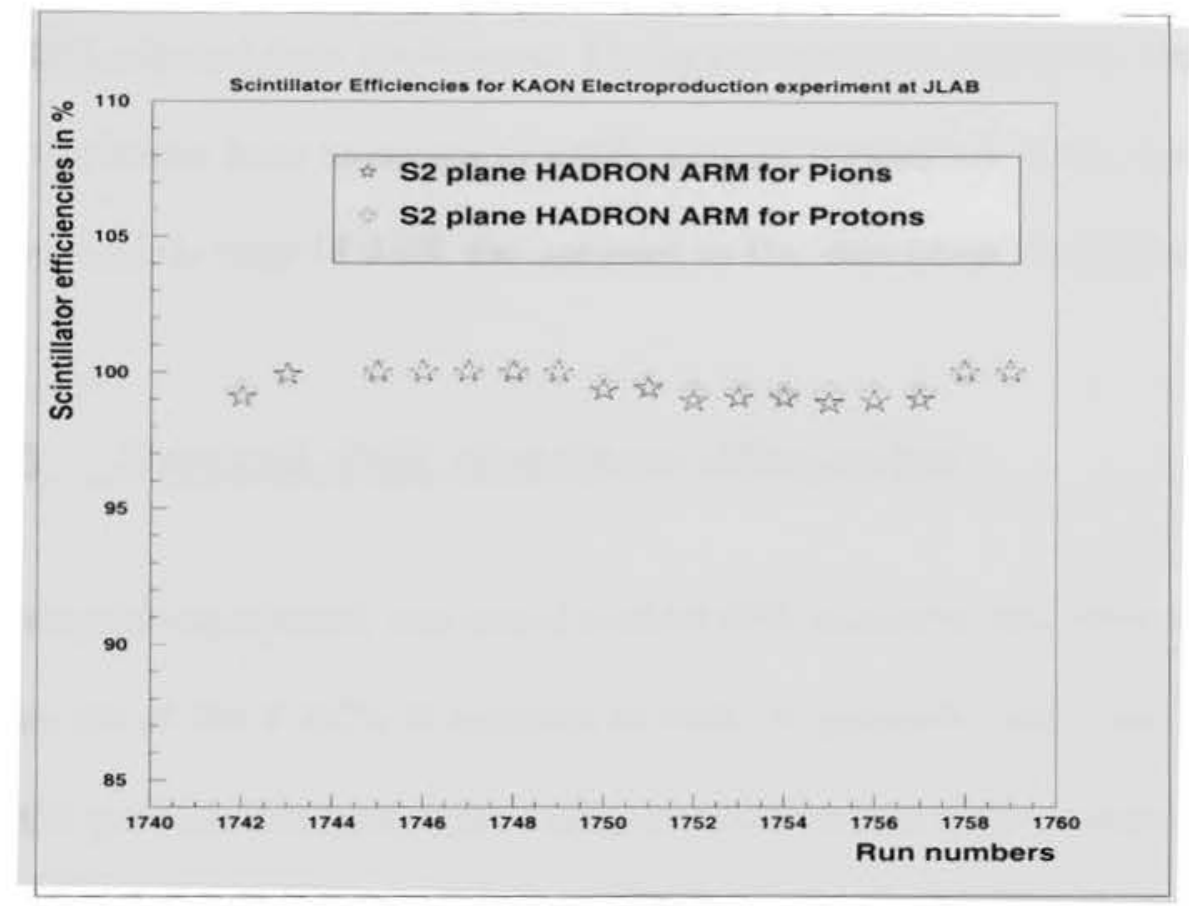

Figure 36: Scintillator efficiencies in the hadron arm ( $S 2$ plane) for different types of particles - protons and pions.

\subsection{Kaon absorption}

The absorption of kaons through the materials encountered in its path [FIS92] [GAV02] was determined using a modified version of the code developed by Joerg Reinhold [REJ03] based on the eikonal approximation [HAU86] and on the kaon proton cross sections as given in Particle Data Book [ABR71] (the code determines the ratio of the real to the imaginary part of the kaon nucleon scattering amplitude for forward angles by fitting the $K$ absorption data on ${ }^{12} \mathrm{C}$ from the Particle Data 
Book. The code was modified to contain all materials found in the kaon's path in the hadron arm and their thicknesses. The kaon absorption correction ranges from $3.65 \%$ for the lowest kaon momenta to $4.66 \%$ for high momentum kaons (see Appendix A). A systematic error of $0.2 \%$ was assigned to the absorption coefficient.

\subsection{Vertical drift chambers efficiencies}

During the experiment, two sets of vertical drift chambers were used, one in each arm. Each set of the $V D C$ 's is essential in order to precisely determine the trajectories of the particles (electrons and hadrons). The vertical drift chambers were built by MIT and Jefferson Lab and were located in front of all other detectors to minimize multiple scattering. To determine the $V D C$ 's efficiency we defined the probability to see a good event through the $i$-th $V D C$ 's plane as the product of the true events and the efficiency of $i$-th plane [BOE02].

$$
P_{\text {GoodEvent }}^{i}=N_{\text {Trues }} \cdot \epsilon_{i}
$$

Another assumption is that the probability to see a good event through any individual $i$ plane is independent of the probability to see a good event through the $j$-th plane, for any $i \neq j$ (the detection efficiency of plane $i$ is independent of the detection efficiency for the $j$-th plane). We defined the $V D C$ efficiencies, epsilon $V_{V D C}^{E, H}$, to be the product of the probability of the detector firing (firing efficiency, $\epsilon_{F i r i n g}^{E, H}$ ) and the 
probability of finding a single track (tracking efficiency, $\epsilon_{\text {Tracking }}^{E, H}$ ) if the detector has fired:

$$
\epsilon_{V D C}^{E, H}=\epsilon_{\text {Firing }}^{E, H} \cdot \epsilon_{\text {Tracking }}^{E, H}
$$

The firing efficiency of the $V D C$ detector was defined as the product of the individual firing efficiencies (the probabilities that each individual plane had fired);

$$
\epsilon_{\text {Firing }}^{E, H}=\prod_{i=1}^{4} \epsilon_{i}^{E, H}
$$

where $\epsilon_{i}^{E, H}$ is the firing efficiency of the $i$-th plane in the Electron or in the Hadron arm. To determine the single plane firing efficiency, $\epsilon_{i}^{E, H}$, of each $V D C_{E, H}$, we considered the multiplicities (number of hits) on each individual $V D C s^{\prime}$ plane. After studying the multiplicity spectra for each individual $V D C$ plane, we concluded that a software cut of $2-20$ in the number of hits per plane includes all possible hits in any of the $V D C$ s' planes. We defined the individual (single plane) firing efficiency (the probability that a plane had fired) [BOE02] as the ratio of the particles that fired all planes and of those that fired at least three planes except the one for which the efficiency is determined

$$
\epsilon_{l}^{\text {Firing }}=\frac{N_{i j k l}}{N_{i j k}}
$$




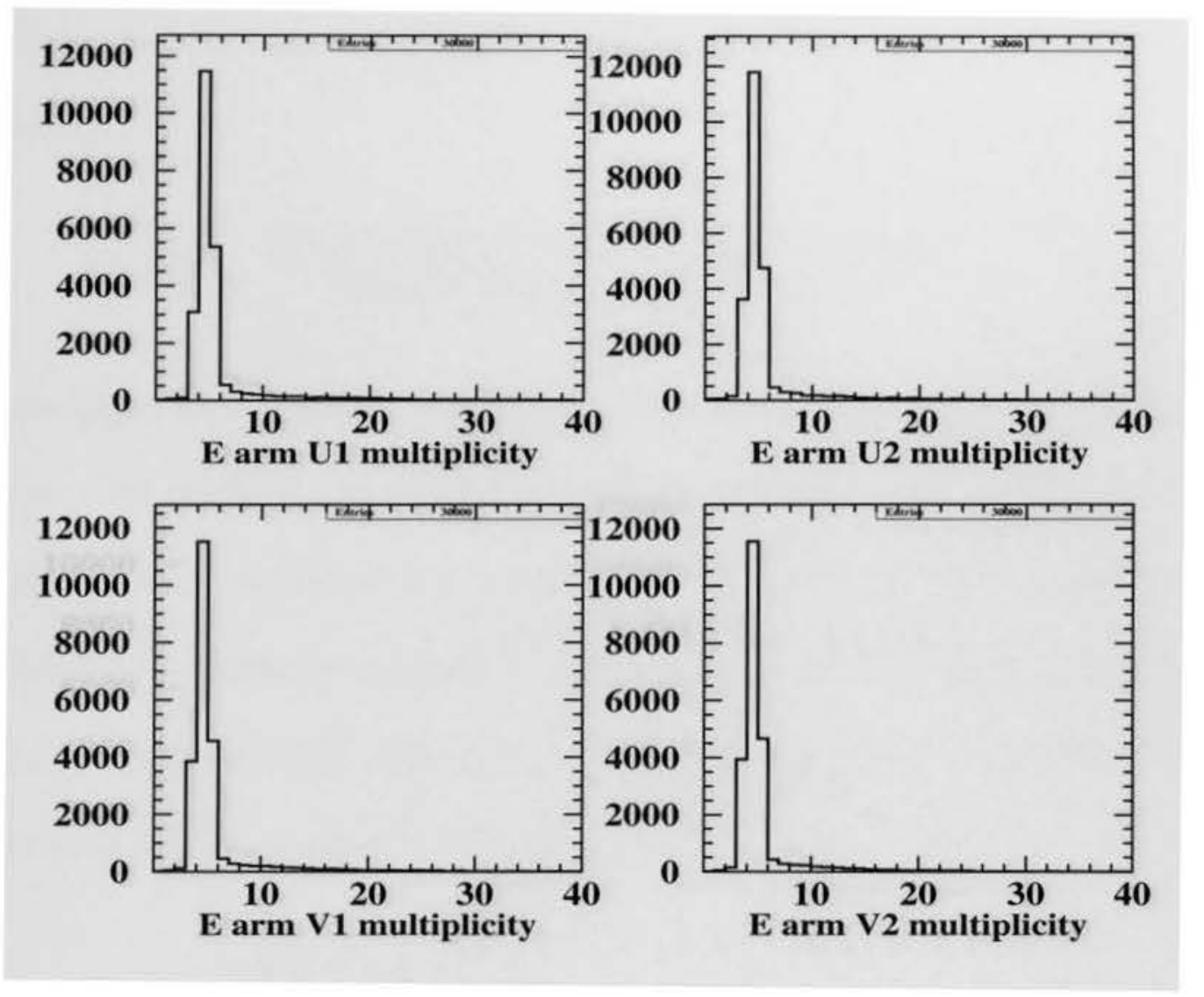

Figure 37: Distribution of the number of hits in $V D C_{\text {Electron }}$.

with the planes' indices fulfilling the inequality condition: $i \neq j \neq k \neq l$. The number of particles that produced a hit in all planes was determined using the multiplicities (number of hits) in each individual plane for a particular $V D C_{E, H}$ :

$$
N_{i j k l}=\text { Mult }_{i} \wedge \text { Mult }_{j} \wedge \text { Mult }_{k} \wedge \text { Mult }_{l}
$$




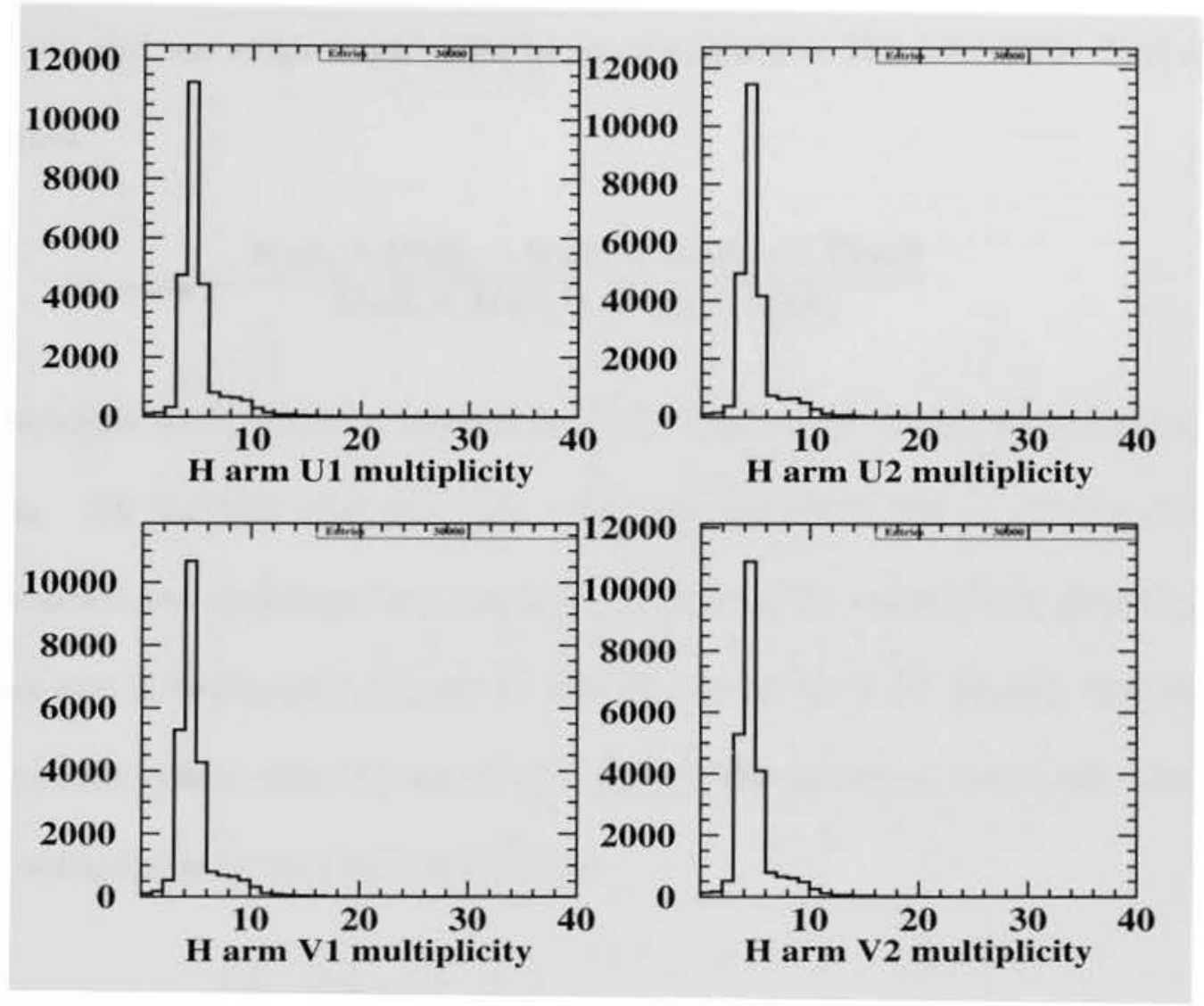

Figure 38: Distribution of the number of hits in $V D C_{\text {Hadron }}$.

with $i$ representing the $U 1$ plane, $j$ the $V 1$ plane, $k$ the $U 2$ plane and $l$ the $V 2$ plane. The number of particles that produced a hit or more in any of the three $V D C$ planes was determined using the formula:

$$
N_{i j k}=\text { Mult }_{i} \wedge \text { Mult }_{j} \wedge \text { Mult }_{k}
$$

for any $i \neq j \neq k$ and $i<j<l$. In the formula above, the plane indices can take any value from 1 to 4 . To determine the tracking efficiency (the probability of finding a 
single track if the detector has fired), we used the ratio of the events that hit all 4 planes and one single track could be reconstructed to the events that fired all four planes:

$$
\epsilon_{\text {Tracking }}=\frac{\text { Mult }_{i} \wedge \text { Mult }_{j} \wedge \text { Mult }_{k} \wedge \text { Mult }_{l} \wedge 1 \_ \text {Track }}{\text { Mult }_{i} \wedge M u l t_{j} \wedge M u l t_{k} \wedge \text { Mult }} \text { }
$$

The single track condition requires one single track to be considered in each separate arm. All particles that fired the $V D C$ and produced two or more tracks were discarded and considered as a tracking inefficiency. The multiplicity distribution for each arm is displayed in Figures 37 and 38. For all the $V D C$ graphs, the statistical errors are smaller than the size of the marker. The statistical errors were calculated by using the error propagation formula:

$$
\sigma_{\epsilon_{V D C}}=\sqrt{\sum_{i=1}^{n}\left(\frac{\partial \epsilon_{V D C}}{\partial x_{i}}\right)^{2} \cdot \sigma x_{i}^{2}}
$$

where $\sigma_{\epsilon_{V D C}}$ is the standard deviation of the $V D C$ s' efficiency, $\frac{\partial \epsilon_{V D C}}{\partial x_{i}}$ is the partial derivative of the efficiency with respect to the variable $x_{i}$ and $\sigma x_{i}^{2}$ are the individual standard deviations of the variables that the efficiency depends upon: $\sigma_{V D C}=$ $f\left(\epsilon_{\text {Tracking }}, \epsilon_{\text {Firing }}\right)$. The overall firing efficiency depends upon the single-plane firing efficiencies, $\epsilon_{\text {Firing }}=f\left(\epsilon_{i}^{E, H}\right)$, and the individual plane firing efficiencies depend upon the number of hits (multiplicities), $\epsilon_{i}^{E, H}=f\left(N_{i j k l}, N_{i j k}\right)$.

In determining the $V D C$ 's efficiency we tried two methods: One in which the firing efficiency was calculated based on four out of four $V D C$ planes (described above) and one in which the efficiency was calculated with a three out of four planes 


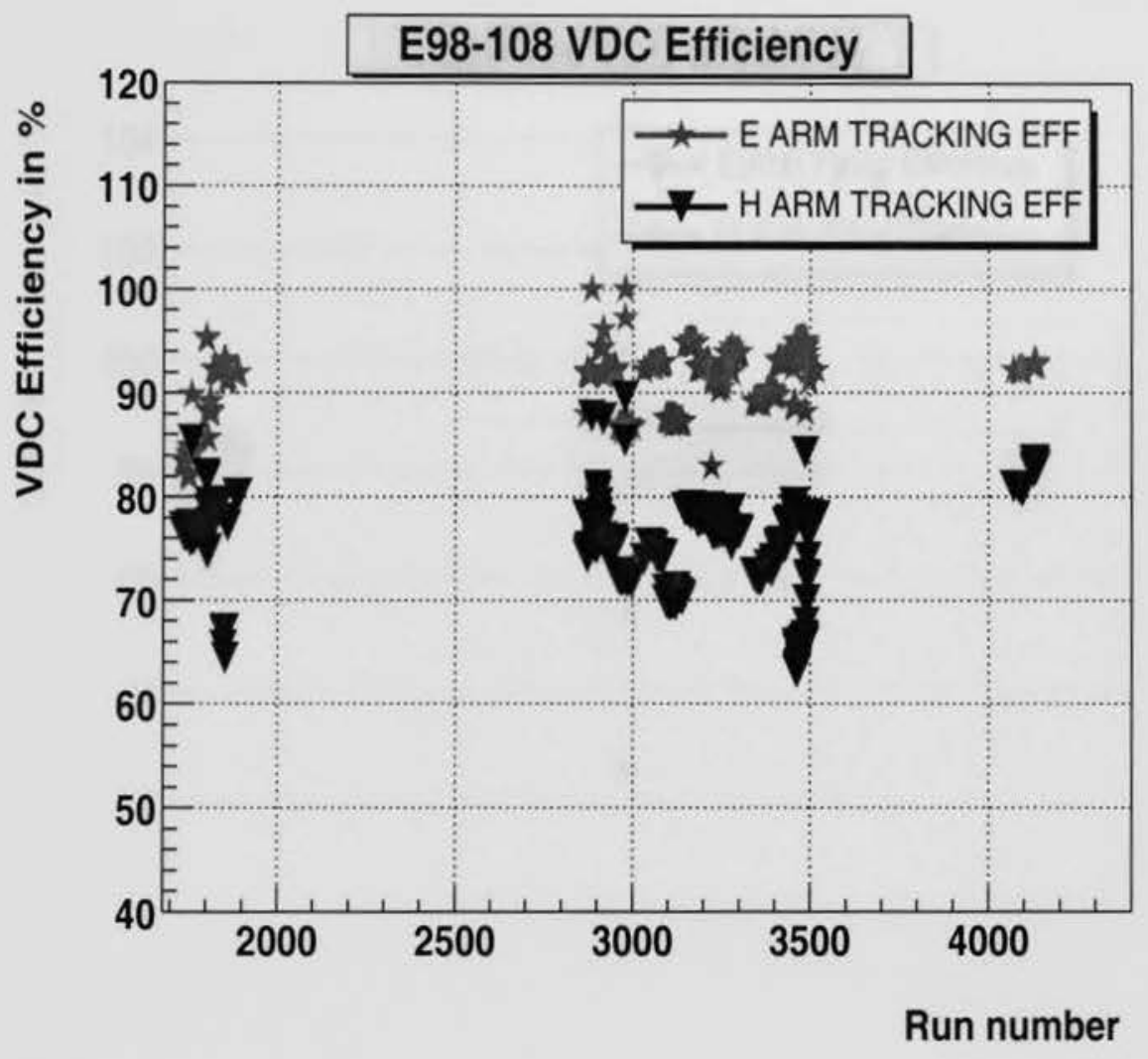

Figure 39: VDCs' Tracking efficiency for E98-108.

approach. In the three out of four planes approach the firing efficiency for each arm is calculated using the formula:

$$
\begin{gathered}
\epsilon_{\text {Firing }}^{E, H}=\frac{N_{1234}}{N_{123}}+\left(1-\frac{N_{1234}}{N_{123}} \cdot \frac{N_{1234}}{N_{134}}\right)+ \\
{\left[1-\frac{N_{1234}}{N_{123}}-\left(1-\frac{N_{1234}}{N_{123}} \cdot \frac{N_{1234}}{N_{134}}\right) \cdot \frac{N_{1234}}{N_{124}}\right]+} \\
{\left[1-\frac{N_{1234}}{N_{123}}-\left(1-\frac{N_{1234}}{N_{123}} \cdot \frac{N_{1234}}{N_{134}}\right)-\left(1-\frac{N_{1234}}{N_{123}} \cdot \frac{N_{1234}}{N_{134}}\right) \cdot \frac{N_{1234}}{N_{124}} \cdot \frac{N_{1234}}{N_{234}}\right]}
\end{gathered}
$$




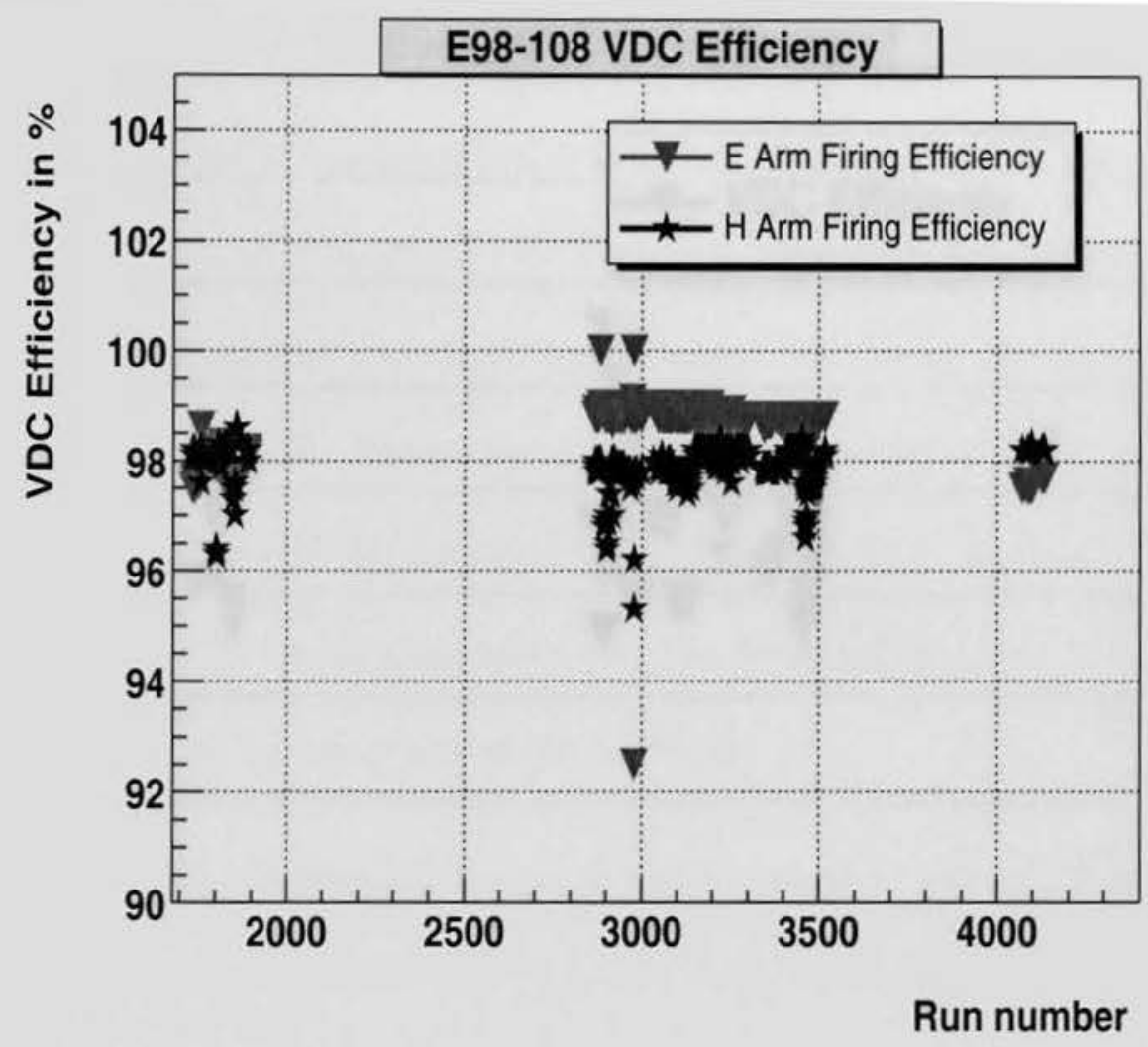

Figure 40: VDCs' Firing efficiency for E98-108.

where $N_{i j k}$ is the number of hits in any of three different $V D C$ planes and $N_{1234}$ is the number of hits in all $4 \mathrm{VDC}$ planes:

$$
N_{1234}=\text { Mult }_{1} \wedge \text { Mult }_{2} \wedge \text { Mult }_{3} \wedge \text { Mult }_{4} .
$$

This method (3 out of 4 planes) for determining the $V D C$ s' firing efficiency leads to a high firing efficiency $(\approx 100 \%)$. The formula for calculating the tracking efficiency in the 3 out of 4 planes method is:

$$
\epsilon_{\text {Tracking }}=\frac{\text { Mult }_{i j k}^{1-\text { Track }}}{M u l t_{i j k}}
$$




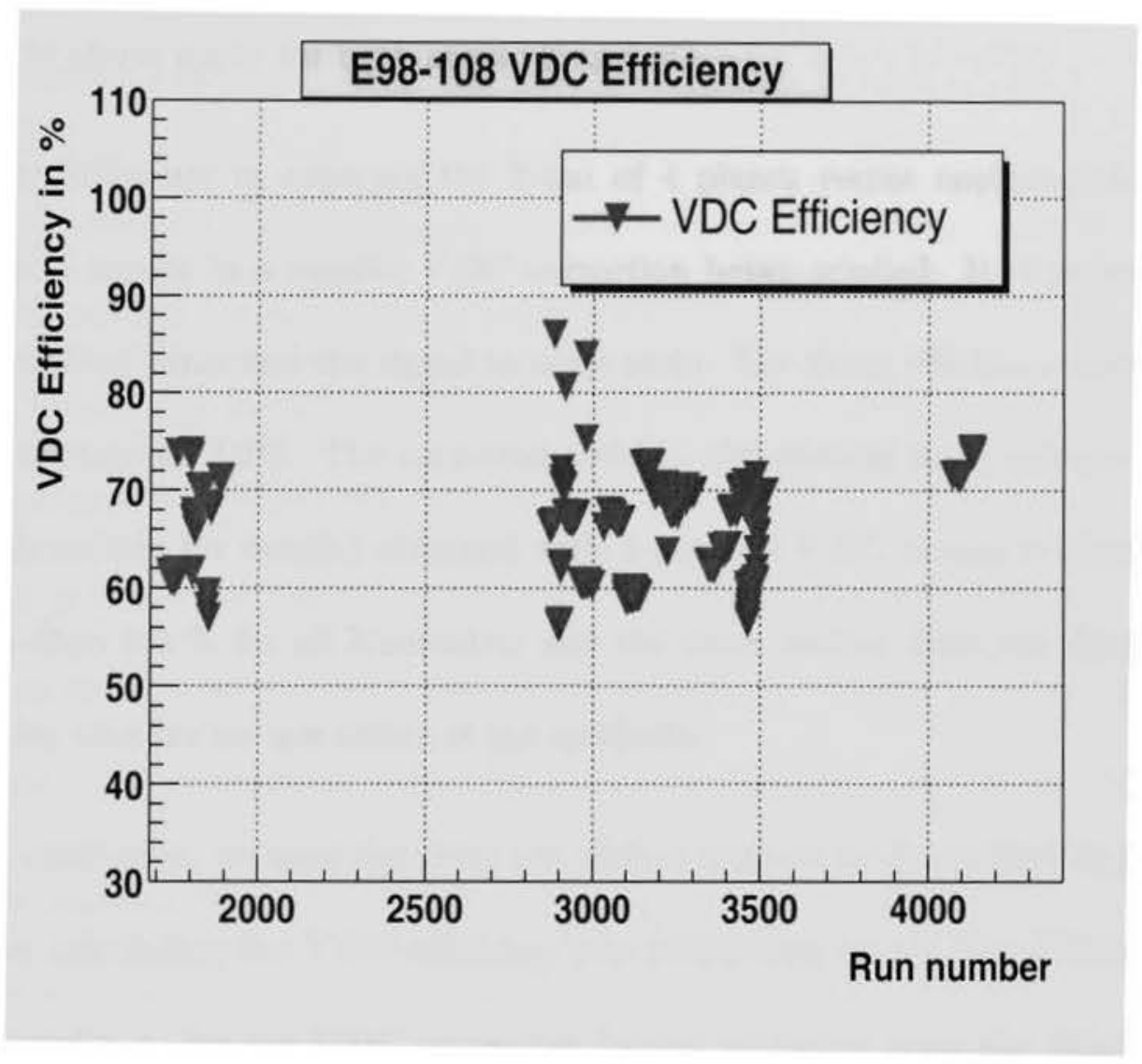

Figure 41: Overall VDCs' efficiency for E98-108.

where $M u l t_{i j k}^{1}$ Track is the number of counts after applying the $O R(\mathrm{~V})$ logical condition between the multiplicities from any 3 planes combination:

$$
P_{123} \vee P_{134} \vee P_{124} \vee P_{234}
$$

with

$$
P_{i j k}=\text { Mult }_{i} \wedge \text { Mult }_{j} \wedge \text { Mult }_{k} .
$$


As previously stated, $M u l t_{i}$ is the number of hits on the $i$-th plane of the $V D C$. The formulas above apply for both arms $(E$ and $H)$.

The difference in applying the 3 out of 4 planes versus applying the 4 out of 4 method results in a smaller $V D C$ correction being applied. It improves (lowers) the statistical error and the signal to noise ratio. The firing efficiency gets improved by an average of $2.2 \%$. The corrected yield in the missing mass spectra (see Data analysis section for details) obtained with 3 out of $4 V D C$ planes is identical down to less than $1.6 \%$ for all kinematics and the cross section does not change within statistics whether we use either of the methods.

In conclusion, we used the three out of four method to determine the systematic error in calculating the $V D C$ efficiency (see subsection 4.5.1). The Tables 10 to 14 , in Appendix A, list the $V D C$ correction factors extracted from the weighted $V D C$ efficiencies. The $V D C$ efficiencies were weighted by the runs' charge for all kinematics (since the $V D C$ efficiency was calculated on a run by run basis and each kinematic consisted of at least 4 runs). The formula used for the total (weighted) $V D C$ efficiency (with the runs' cumulated charge as the weight factor) is:

$$
\epsilon_{V D C}^{\text {Total }}=\frac{\sum_{i=1 \text { st run }}^{\text {Last run }} \epsilon_{V D C} \cdot Q_{i}}{\sum_{i=1 \text { st run }}^{\text {Last run }} Q_{i}}
$$

where $Q_{i}$ is the charge of run $i$. 


\subsection{Efficiency determination for the 1st aerogel Cerenkov}

\section{detector}

In order to determine the efficiency to kaons for the 1st Aerogel Cerenkov detector, we assumed that kaons behave as protons from the point of view of the interaction within the aerogel media. The assumptions is based on the similarity between kaon cross sections and proton cross sections [EID04]. Since $A 1$ is not supposed to fire on protons or kaons (see Fig. 17 representing the threshold velocities for $A 1$ and $A 2$ ) by selecting the protons that fired the aerogel detector we actually selected the knock-on electrons ( $\delta$ rays) generated by protons in their interaction with the aerogel detector.

We determined the A1 efficiency for proton detection by selecting a clean sample of protons in the corrected (corrected for path length difference with respect to the central rays [ESP02]) coincidence time spectra, $T C_{C O R}$. Even though the $T C_{C O R}^{p}$ and $T C_{C O R}^{\pi}$ peaks were clearly separated we assumed our proton sample could have some contamination (pions). So we had to exclude the pions that fall underneath the protons' peak in the coincidence time spectra, $T C_{C O R}^{p}$. In order to accomplish this we have applied a logical $A N D$ condition between the $T C_{C O R}^{p}$ and the logical negation of the second aerogel detector, $\overline{A 2}$ :

$$
\text { Clean }_{\text {protons }}=T C_{C O R}^{\text {protons }} \wedge(\overline{A 2})
$$

However, some pions will still fall into this region and we have to account for them. They are obviously not coincidence pions, or they would have been excluded with the 
$T C_{C O R}^{p}$ cut. There are not many accidentals under the proton time of flight peak, and the majority are accidental protons (due to applying the A2 cut). But even a handful of pions might matter, so we estimated how many pions there were. We handled the accidental pions in two steps.

First, we determined the A2 efficiency for pions by applying a narrow $T C_{C O R}$ cut on pion time of flight, $( \pm 1 \sigma)$. Then we applied a cut of $4 P E$ on $A 1$. This $4 P E$ cut selected the pions that produced at least four photoelectrons in $A 2$, although of course it could have a small contamination of other particles ( $\delta$ rays are very unlikely to produce $4 P E$ in $A 2$, they generate at most 1 or $2 P E$ ); we used this as a "clean" sample of pions. Then we determined how many of these "clean" pions generated a signal in $A 2$.

As a second step, we determined how many pions per ADC channel are under the proton TOF peak without applying a cut on the number of photoelectrons in $A 2$. We determined how many events inside the $T C_{C O R}^{p}$ cut produced more than 1.5 $P E$ in $A 1$. Of course some of protons that generated more than $1.5 P E$ (which was a standard $A 1$ cut in our analysis) are not pions but instead are $\delta$ rays produced by protons, but it is a very small percentage (smaller than $0.1 \%$ ) that we can ignore. The formula for A1 efficiency to protons in $\%$ is:

$$
\epsilon_{A 1}^{p}=100 \times \frac{\left(Y_{T C^{p r o t o n s}}^{A 1 \wedge(\neg A 2)}-Y_{\pi}^{A 1}\right)}{\left(Y_{T C_{p}}^{A 1}-Y_{\pi}^{A 1}\right)}
$$

where $Y_{T C^{\text {protons }}}^{A 1 \&(\neg A 2)}$ represents the protons' yield in the corrected coincidence time of flight, protons that fired $A 1$ with the standard cut $(A 1 \in(150,6000)$ channels $)$ but 
it was less than five channels in $A 2, Y_{T C_{p}}^{A 1}$ represents the protons yield of the protons that fired the $A 1$ detector and generated between $4 P E$ and $60 P E$ and $Y_{\pi}^{A 1}$ is the number of pions that fired $A 1$ out of total clean pions under the protons time of flight:

$$
Y_{\pi}^{A 1}=\left[\frac{Y_{A 2}^{\pi}}{\text { Clean }_{\pi}}\right] \times A 1_{\pi}
$$

with $Y_{\pi}^{A 1}$ being the yield of $\pi$ under the protons' corrected coincidence time peak, $Y_{A 2}^{\pi}$ are the $\pi$ in the pions' $T C \_C O R$ peak and $C_{-} l e a n_{\pi}$ are the clean pions. In terms of the cuts applied the quantities are as follows:

$$
\begin{aligned}
& Y_{\pi}^{A 1}=T C^{\text {protons }} \wedge R F N \wedge A 1 \in(150,6000) \wedge T \text { woarm } \_x \\
& Y_{A 2}^{\pi}=T C^{\pi} \wedge R F N \wedge A 1 \in(400,6000) \wedge T \text { woarm } x \wedge A 2(<0.005) \\
& \text { Clean } n_{\pi}=T C^{\pi} \wedge R F N \wedge A 1 \in(400,6000) \wedge T \text { woarm_x } \\
& A 1_{\pi}=T C^{\text {protons }} \wedge R F N \wedge A 1 \in(150,6000) \wedge T \text { woarm_x }
\end{aligned}
$$

The low cut on $A 2$ was applied in order to exclude the pions. Here $T C^{\text {protons }}$ is a cut on the corrected coincidence time around the proton peak, $R F N$ represents the standard $R$ - function cut used for the entire E98-108 analysis (see Section 4.3), and Twoarm $x$ represents a cut on the $x$ coordinate of the crossing of the two spectrometer vertexes. The $R$ - function cut was applied in order to ensure that only particles coming into the physical acceptance are considered. We have also checked that no 
particles are discarded (lost) by determining the yield of the true coincident protons, after accidentals and coincident $\pi$ subtraction. We compared the protons' yield that fired the $A 1$ detector generating between $1.5 P E$ and $60 P E$ (the standard $A 1$ cut). The determination was made for both running periods 2001 and 2002. The results are shown in the Table 3 below. One can clearly see there were no discards, no protons lost.

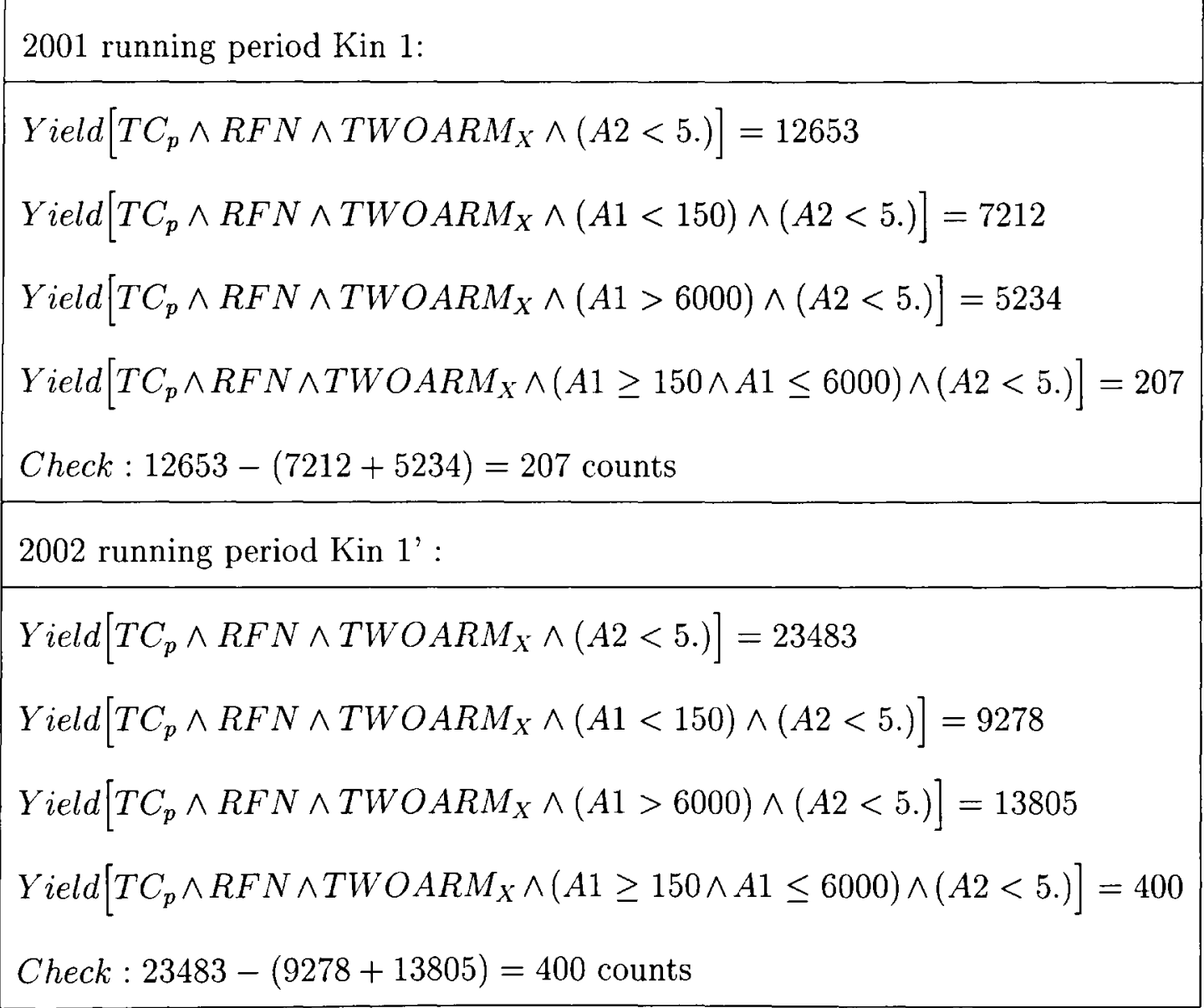

Table 3: Protons yield calculations for $A 1$ efficiency determination. 
The $A 1$ efficiency results are presented in Fig. 42. A least square fit (using a first degree polynomial) of the $A 1$ efficiency versus kinematic number is presented in Fig. 42 .

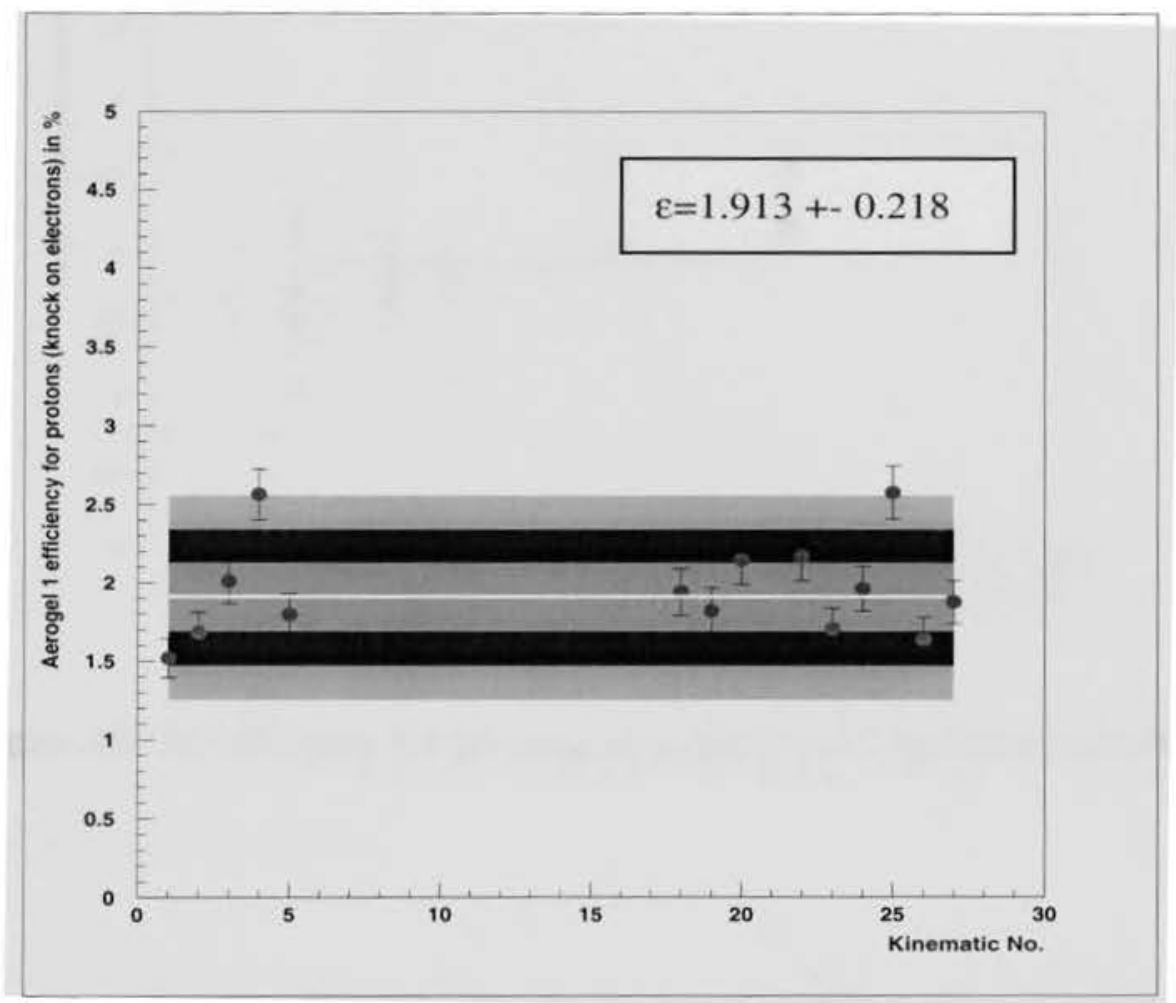

Figure 42: A1 efficiency for protons (knock-on electrons), first degree polynomial fit together with $1 \sigma, 2 \sigma$ and $3 \sigma$ bands.

Note that all points in Fig. 42 are within three standard deviations. A1 efficiency for protons (knock-on electrons) as a function of hadrons' momenta is presented in Fig. 43. From this graph, 42, we deduced the systematic uncertainty in determining the $A 1$ efficiency to kaons as being $0.57 \%$ since we assumed, in the absence of any 


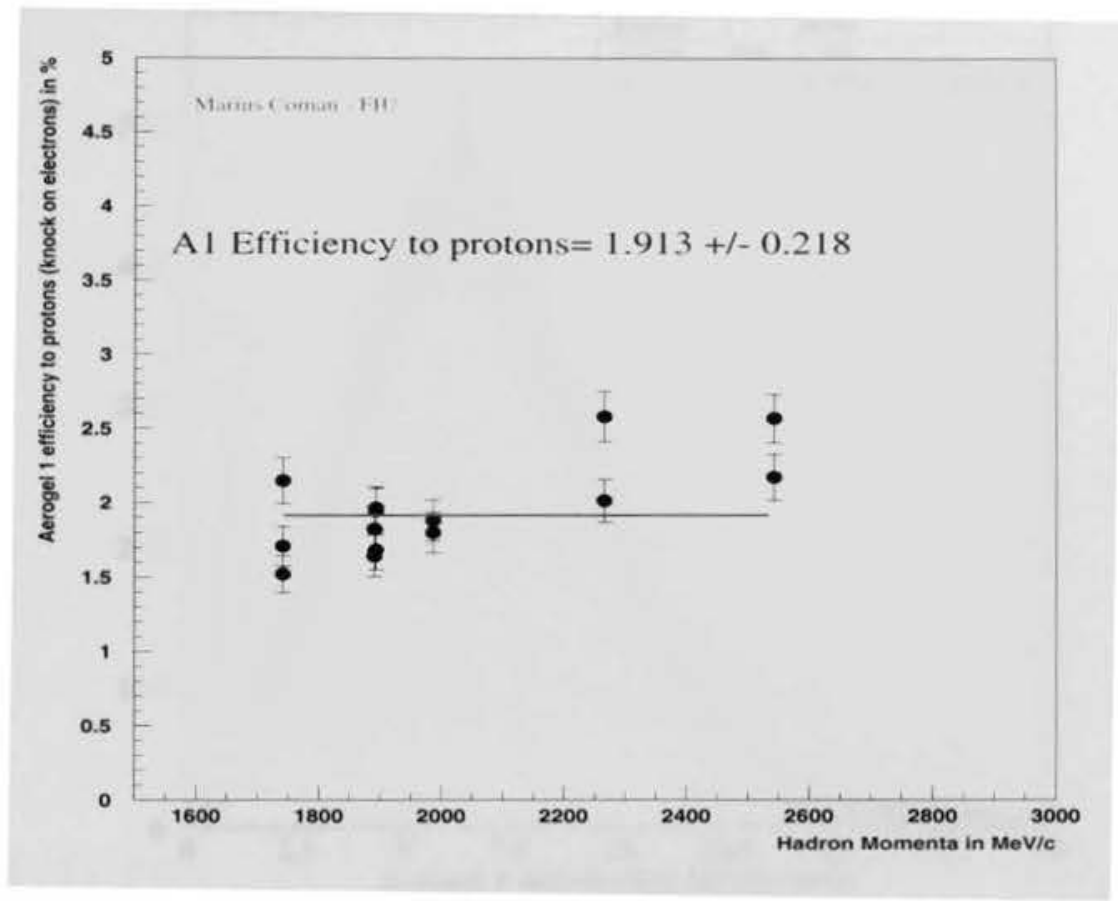

Figure 43: A1 efficiency for protons as a function of hadrons' momenta.

efficiency loss, the $A 1$ efficiency should be reproducible. We considered for this calculation the $A 1$ efficiency for kaons from kinematic 3 and kinematic 25 (the maximum percent difference) where the kaon momenta was $2266 \mathrm{MeV} / \mathrm{c}$ and the efficiencies were $97.43 \%$ respectively $97.99 \%$.

We have considered it as systematic uncertainty based on the initial assumptions we made that protons behave as kaons.

As I have mentioned earlier, the E98-108 experiment took data in both 2001 and 2002. A problem that we encountered was a change in the efficiency of both 


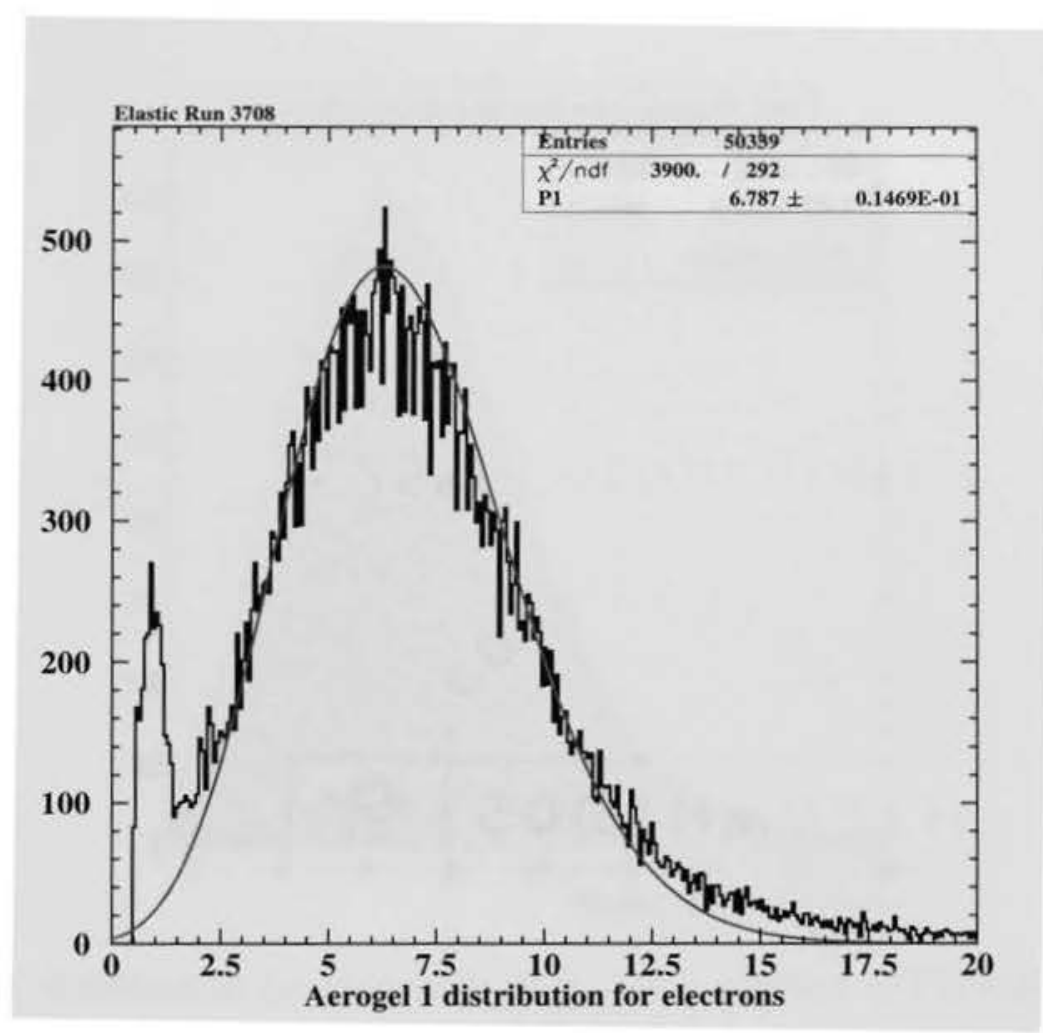

Figure 44: A1 distribution for electrons, 2001; The position of the single photoelectron peak is shown.

aerogel detectors. In 2001 the number of photoelectrons generated by hadrons in $A 1(n=1.015)$ was 6.3 photoelectrons $(P E)$. The $A 1$ distribution for electrons $(\beta \approx 1)$,as obtained in 2001 , together with a Poisson fit is presented in Fig. 45 . The $2001 A 1$ distribution for $\pi(\beta \approx 1)$ together with a Poisson fit is presented in Fig. 46. The $A 1$ distribution for $\pi(\beta \approx 1)$ together with a Poisson fit from 2002 running period is presented in Fig. 47 .

One can clearly see that over a period of 1 year, the mean number of $P E$ generated by pions having the same momenta, $1740 \mathrm{MeV} / \mathrm{c}$ decreased from 6.27 


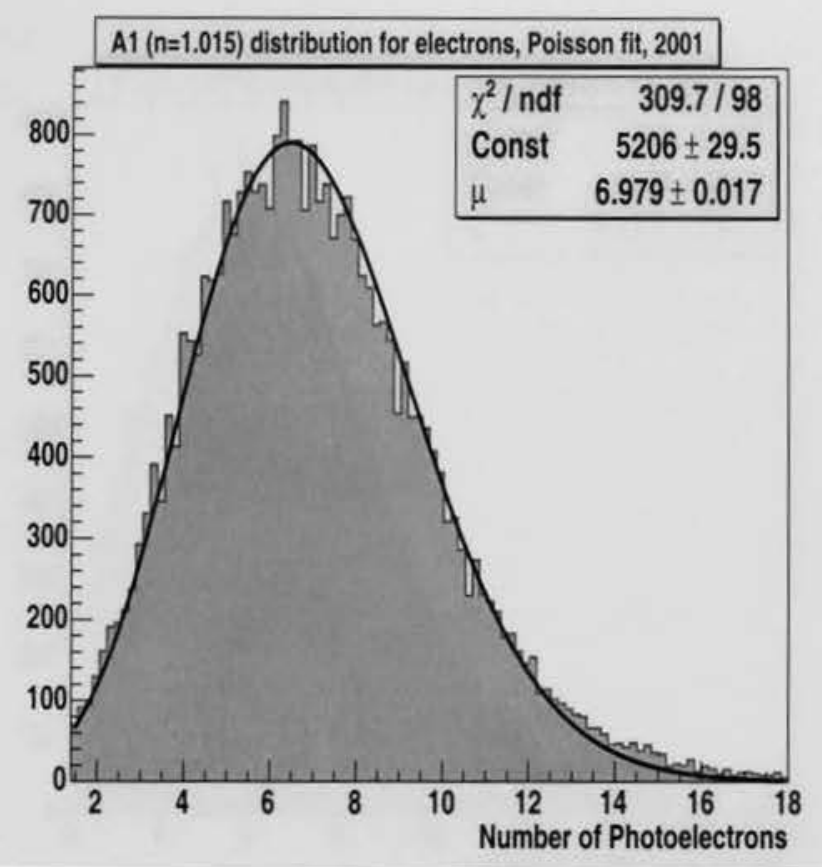

Figure 45: A1 distribution for electrons, 2001, Mean number of PE from Poisson fit $=6.98 \pm 0.017$

to 4.31 , a $31.3 \%$ percentage decrease. The decrease in the mean number of photoelectrons produced by $\pi$ in $A 1$ was not due to the high voltage at which the Burle photomultipliers were operated but due to the aging of the PMTs and/or reflective millipore layer [WOJ02]. To take into account this effect for all data in 2002, the database was recalculated, and, the gains of each individual PMT determined by fitting the individual spectra and finding the pedestals and means (for each individual PMT for the $A 1$ detector). With the new values for the gains and pedestals, a new database was created and used to analyze all 2002 runs. 


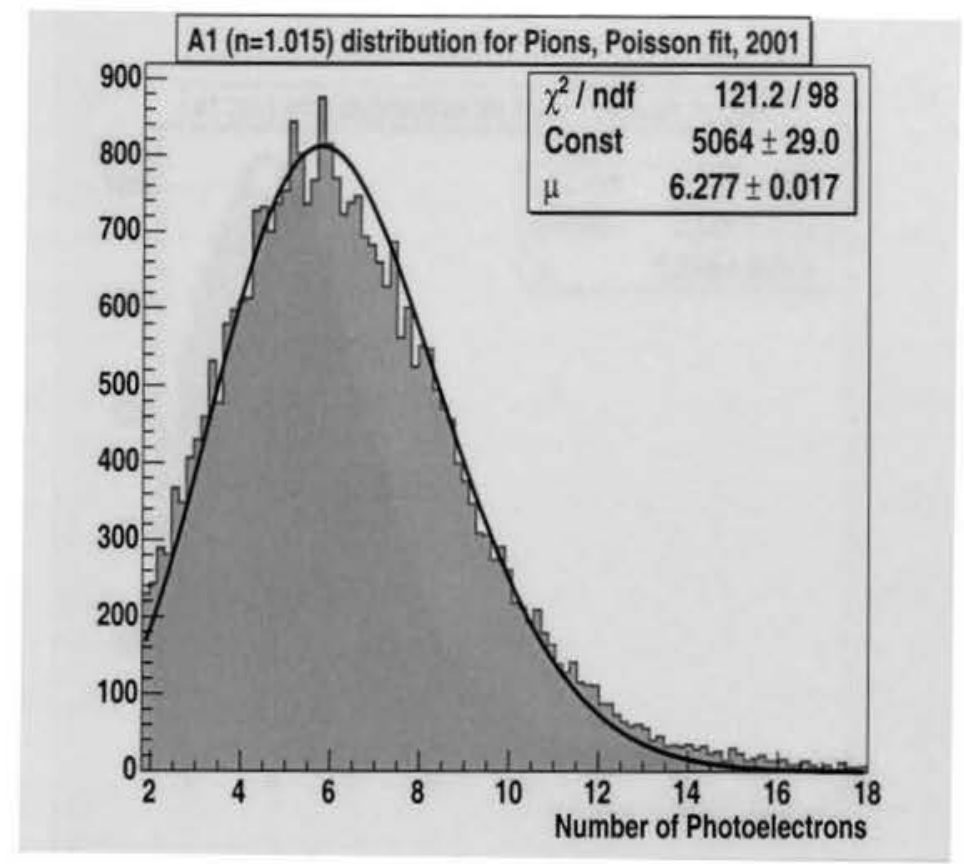

Figure 46: A1 distribution for $\pi$, 2001, Mean number of PE from Poisson fit $=$ $6.28 \pm 0.017$

\subsection{Efficiency for the 2nd aerogel Čerenkov detector}

The efficiency for the 2nd aerogel Čerenkov detector, $A 2$, used to detect both $\pi$ and $K$ was determined using a dedicated program that calculates the probability to exclude a certain number of photoelectrons (when applying a specific cut on the number of PE in the Aerogel detector).

The program makes the assumption that the average number of photoelectrons in $A 2$ is Poisson distributed and it considers only one type of particles for the 


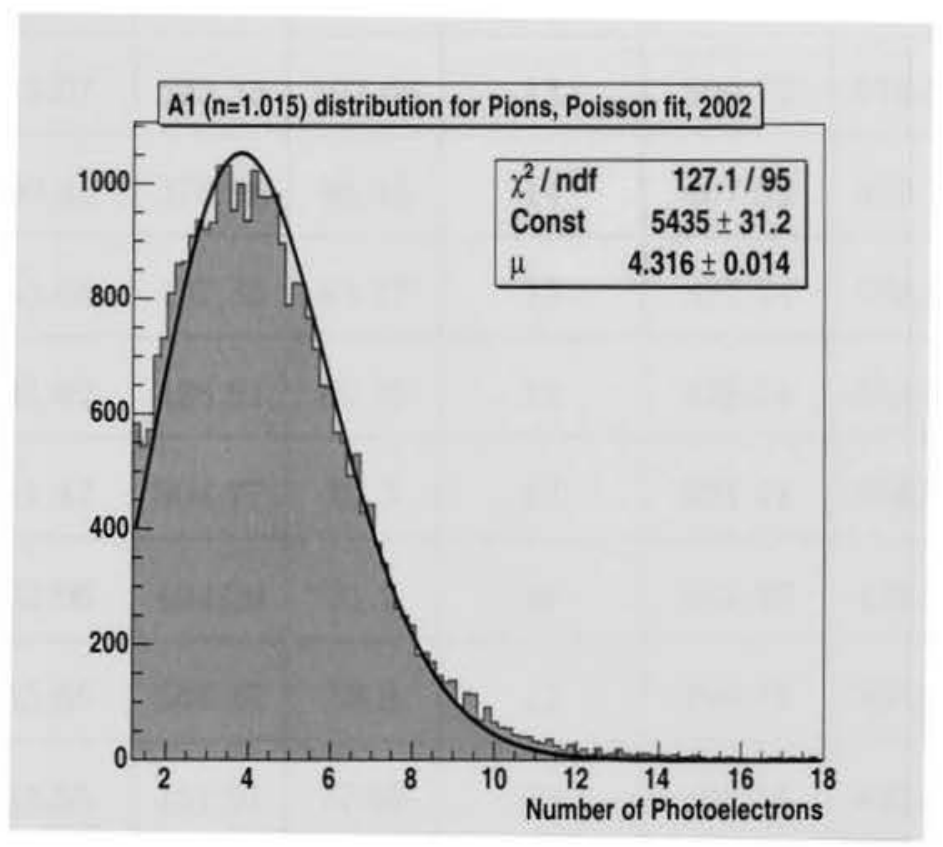

Figure 47: A1 distribution for $\pi, 2002$, Mean number of PE from Poisson fit $=$ $4.32 \pm 0.014$

calculation. The code does not consider the case in which the average number of photoelectrons is generated by two or more particles at a time.

As mentioned in Section 3.6, we were faced with an efficiency loss of both aerogel detectors. In 2001 the average number of photoelectrons generated by $\pi$ in $A 2(n=$ 1.055) was about 28.2 photoelectrons (see Fig. 48) while in 2002 the average number of $P E$ generated by pions having the same momenta was about 20.9 (see Fig. 52) indicating a percent loss of $\approx 26 \%$.

The decrease in the mean number of photoelectrons generated by hadrons in $A 2$ was mainly due to the aging of the PMTs and/or reflective millipore layer. 


\begin{tabular}{|c|c|c|c|c|c|c|c|}
\hline PMT no. & Pedestal & Mean & Gain & PMT no. & Pedestal & Mean & Gain \\
\hline 1 & 415.67 & 523.33 & 107.66 & 13 & 500.72 & 578.39 & 77.67 \\
\hline 2 & 290.45 & 375.90 & 85.45 & 14 & 407.62 & 492.18 & 84.56 \\
\hline 3 & 352.68 & 437.85 & 85.17 & 15 & 437.24 & 522.83 & 85.59 \\
\hline 4 & 341.62 & 428.61 & 86.99 & 16 & 428.58 & 514.58 & 86 \\
\hline 5 & 411.47 & 504.77 & 93.3 & 17 & 321.41 & 398.37 & 76.96 \\
\hline 6 & 403.06 & 494.26 & 91.2 & 18 & 341.43 & 425.54 & 84.11 \\
\hline 7 & 455.65 & 544.45 & 88.8 & 19 & 290.13 & 366.84 & 76.71 \\
\hline 8 & 353.55 & 431.21 & 77.66 & 20 & 359.16 & 442.11 & 82.95 \\
\hline 9 & 390.06 & 465.33 & 75.27 & 21 & 401.08 & 492.31 & 91.23 \\
\hline 10 & 363.21 & 460.48 & 97.27 & 22 & 382.36 & 455.00 & 72.64 \\
\hline 11 & 363.00 & 442.68 & 79.68 & 23 & 468.75 & 540.41 & 71.66 \\
\hline 12 & 328.67 & 396.33 & 67.66 & 24 & 385.54 & 469.54 & 84 \\
\hline
\end{tabular}

Table 4: Pedestal and gains for $A 1$ Čerenkov detector, 2002

To take into account the aging of the PMTs for all data in 2002 we had to rebuild the database as specified in 3.6 , recalculate the gains of each individual PMT by fitting the individual spectra and finding the pedestals and mean). For all kinematics taken at the same hadron momenta the same cut was applied on the number of $P E$ generated by kaons in $A 2$. The $A 2$ correction factors are listed in Appendix 5.1 for all kinematics. 


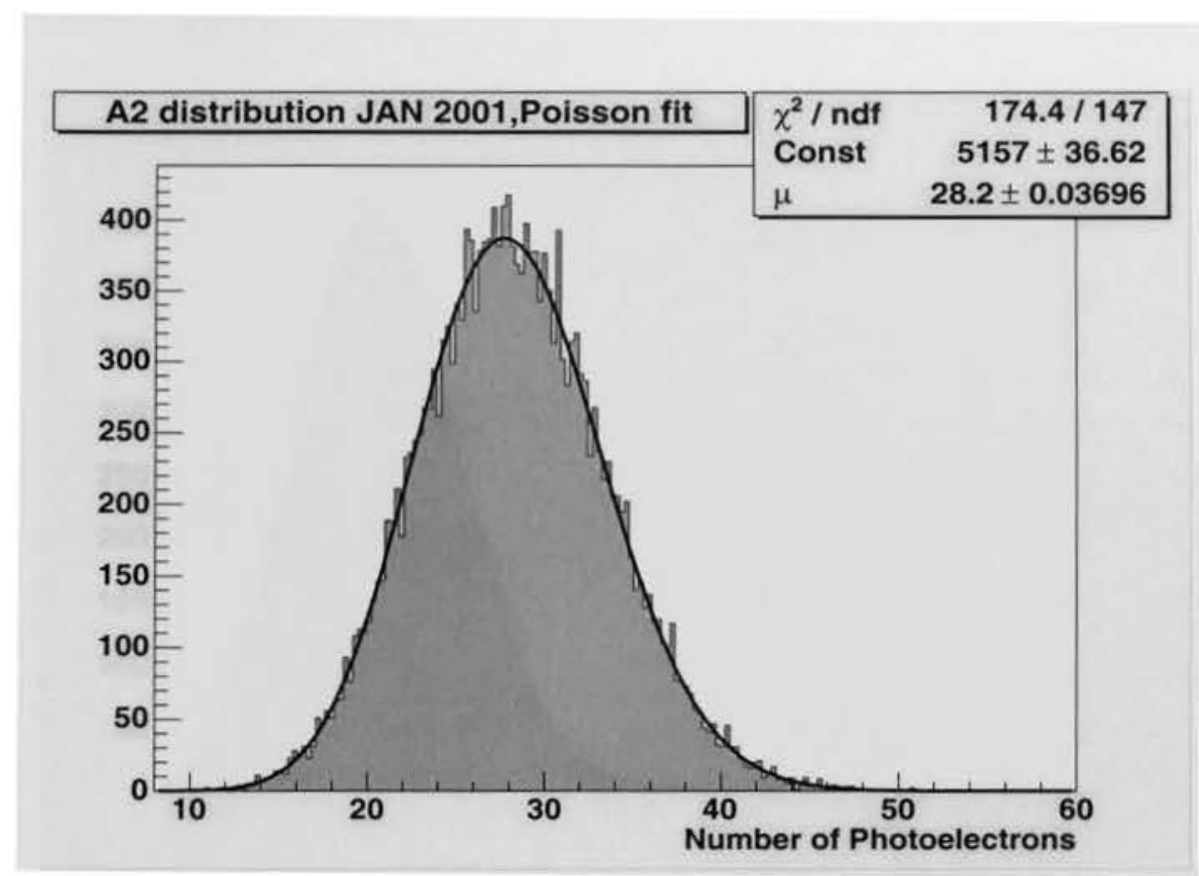

Figure 48: $A 2$ Čerenkov detector: distribution of number of $P E$ for $\pi$, Kin 1, 2001.

The C code calculates the percentage of $K$ and/or $\pi$ excluded from $A 2$ and takes as input the experimental average number of $P E$ determining the cumulative distribution function $F(n, \mu)$ according to the formula :

$$
F(n, \mu)=\sum_{i=0}^{n}\left[\frac{e^{-\mu} \cdot \mu^{i}}{i !}\right]
$$

based on the Poisson distribution function [LYO99]:

$$
P\left(n, \mu_{P E}\right)=\frac{\mu_{P E}^{n}}{n !} \cdot e^{-\mu_{P E}}
$$

where $\mu_{P E}$ is the mean number of $P E$ generated in $A 2$, and $n$ represents the cut applied on the number of $P E$. 


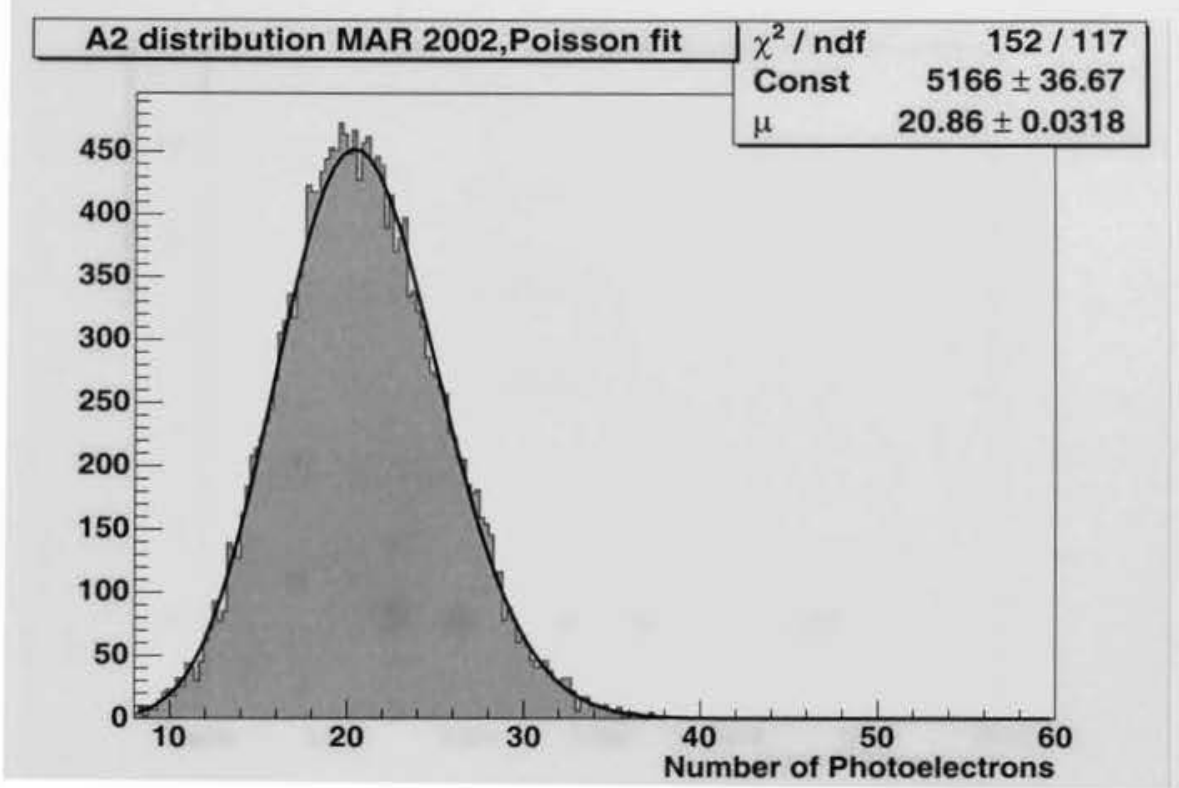

Figure 49: $A 2$ Čerenkov detector: distribution of number of $P E$ for $\pi$, Kin 1 prime, 2002

A second approach to establish the $A 2$ inefficiency to kaons was to determine the number of kaons excluded based on the average number of $P E$ obtained in $A 2$ with particles having $\beta \approx 1$ (electrons). The efficiency was thus normalized to the average number of $P E$ obtained with $\beta=1$ particles. A separate $\mathrm{C}$ code was developed to determine the theoretical average number of $P E$ for particles other than $e$, in our case $\pi$ and $K$. The drawback of this method is that it does not consider the experimental average number of $P E$ generated by $K$, inferring it instead from the average number of photoelectrons, $\mu_{P E}$, produced by $e$, and does not take into consideration the efficiency loss (mentioned above) over time. 


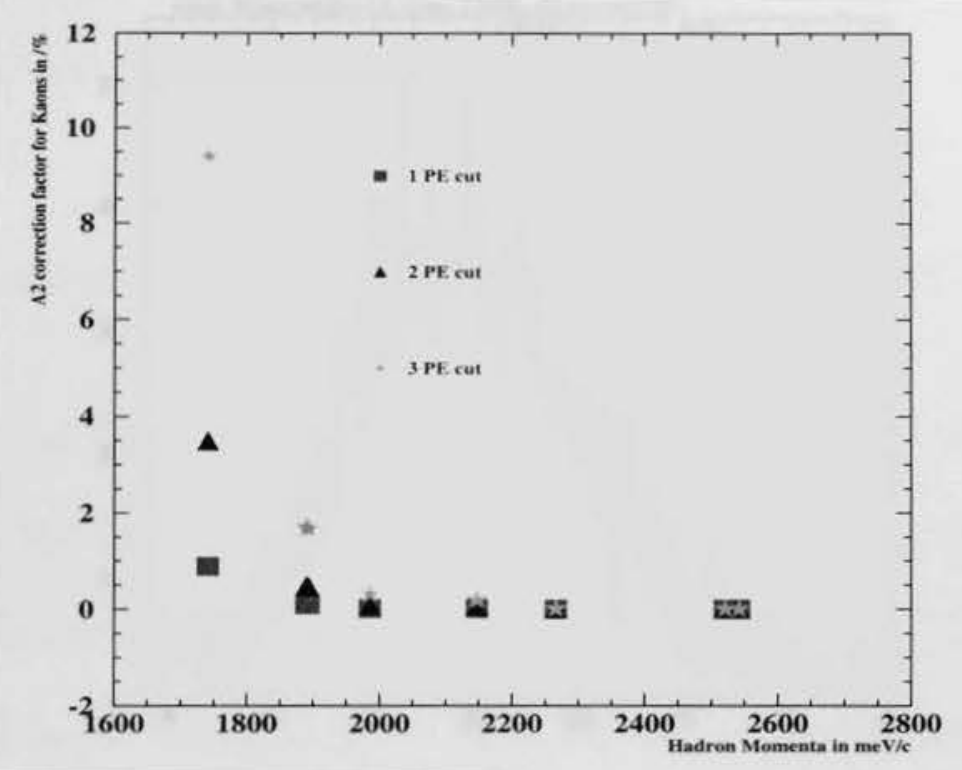

Figure 50: $A 2$ correction factor for a 1 to $3 P E$ cut as a function of particles' momenta; a 2 to $4 P E$ cut was used for all kinematics.

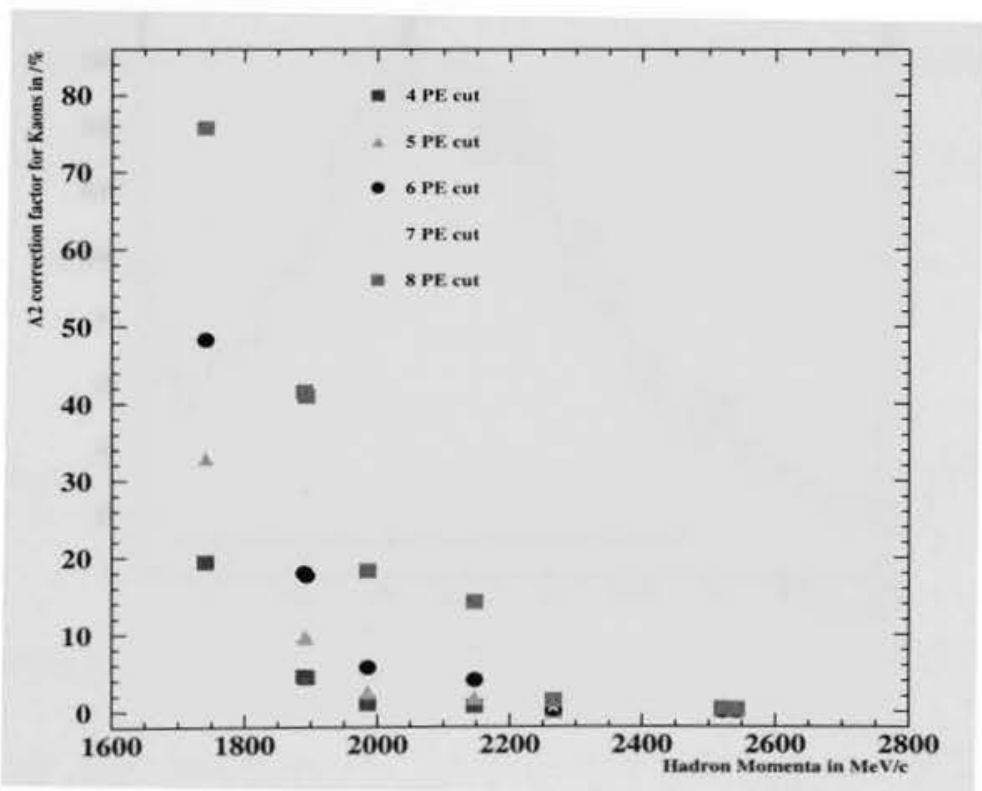

Figure 51: $A 2$ correction factor for a 4 to $8 P E$ cut as a function of particles' momenta. 


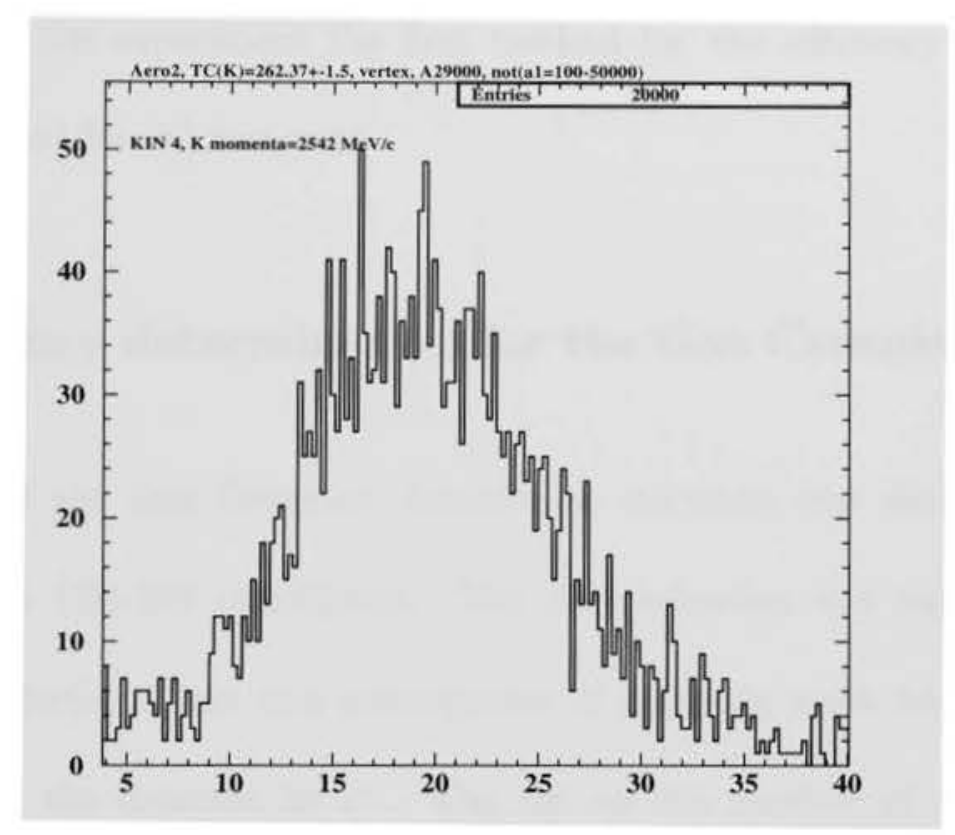

Figure 52: $A 2$ Čerenkov detector: distribution of number of $P E$ for kaons, Kin 4.

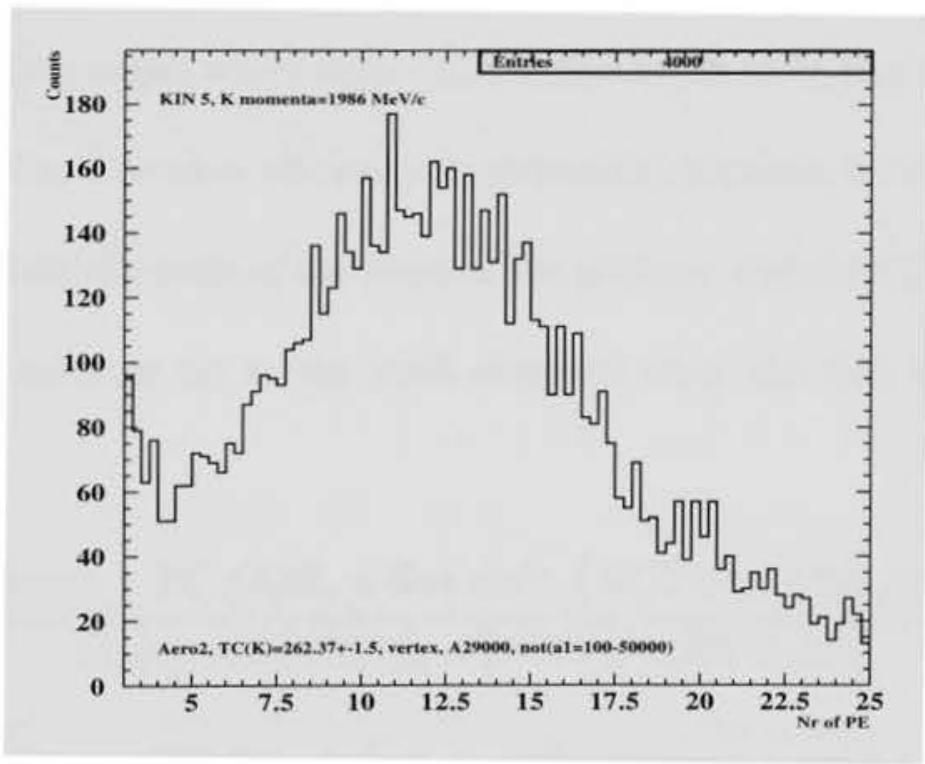

Figure 53: $A 2$ Cerenkov detector: distribution of number of $P E$ for kaons, Kin 5. 
For the E98-108 experiment the first method for the efficiency determination (correction factors) for $A 2$ was used.

\subsection{Efficiency determination for the Gas Cerenkov detector}

The efficiency of the Gas Cerenkov detector to electrons was determined for all momenta for the E98-108 experiment. The determination was made in order to correct for the electrons lost as a consequence of applying a cut on the number of $P E$ produced in the detector by $e^{-}$. The cut on the number of $P E$ in the Gas Cerenkov detector was applied in order to exclude the $\pi^{-}$especially at backward electron scattering angles where their contribution tends to be significant. In order to determine the Gas Cerenkov efficiency for detecting electrons, we selected coincident electrons and took the ratio of the yield in the protons' time of flight peak (with the Gas Čerenkov detector in) to the yield obtained when the Gas Čerenkov detector was out:

$$
\epsilon_{\text {Gas }}=\frac{\text { Event }_{5} \wedge T C_{-} C O R_{p} \wedge \text { Gas_cut } \wedge(N O T(A 1 . A N D . A 2))}{\text { Event }_{5} \wedge T C_{-} C O R_{p} \wedge(N O T(A 1 . A N D . A 2))}
$$

where the $\operatorname{NOT}(A 1 . A N D . A 2)=\overline{(A 1>150)} A N D \overline{(A 2>200)}$ is the logical negation of both $A 1$ and $A 2$ detectors (since neither is supposed to fire on protons). In Eq. 38 we have selected the coincidence electrons by applying a logical $A N D$ condition between the protons in the coincidence time spectra, $T C_{-} C O R_{p}$, the gas Čerenkov and the coincidence events, Event ${ }_{5}$. The Gas Čerenkov cut, Gas_cut, used in the efficiency analysis was the standard cut on the number of $A D C$ channels used 


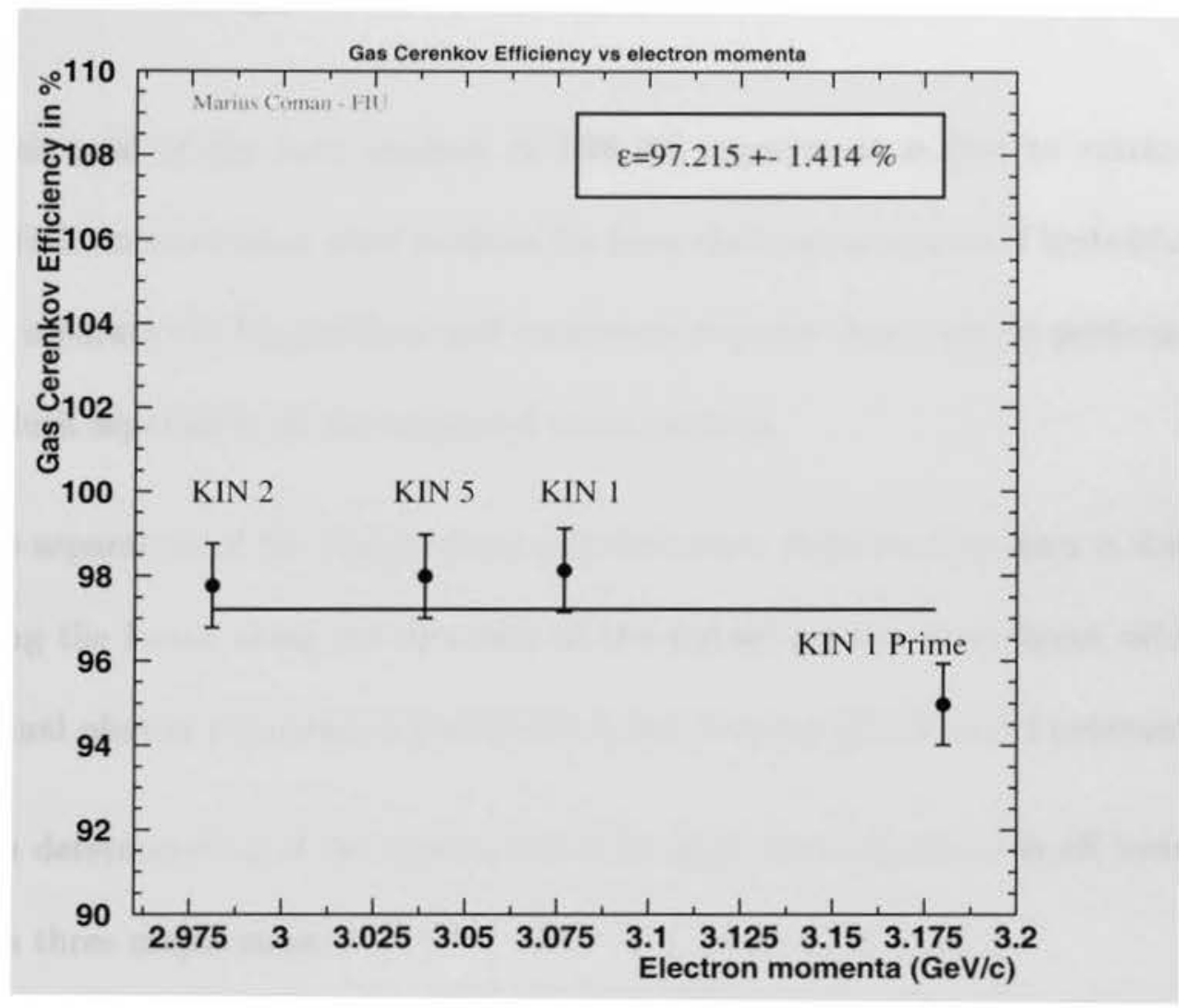

Figure 54: Gas Čerenkov efficiency for electrons as a function of particles' momenta

for the entire E98-108 analysis: $150<A D C_{G a s}<50000$. The efficiency was found to be consistent within statistics for all momenta and the result of fitting the efficiencies with a first degree polynomial is $97.21 \pm 1.41 \%$ (see Fig. 54). 


\section{Data analysis}

The main goal of the data analysis in E98-108 experiment is first to extract the differential center-of-mass cross sections for kaon electroproduction off hydrogen and then to separate the longitudinal and transverse response functions by performing a Rosenbluth separation on the extracted cross sections.

The separation of the longitudinal and transverse response functions is done by detecting the kaons along the direction of the virtual photon at different values of the virtual photon polarization parameter $\epsilon$, but keeping $Q^{2}, W$ and $t$ constant.

The determination of the cross sections for kaon electroproduction off hydrogen involves three major steps:

1. Identifying the particles in the reaction and subtracting the unwanted contributions; obtaining the $\Lambda$ and $\Sigma$ missing mass spectra and calculating the corresponding yields.

2. Determining the correction factors (kaon absorption, coincidence time cuts) and detectors' efficiencies.

3. Calculating the cross section using the information obtained above. 


\subsection{Particle identification}

Particle identification is required to distinguish the kaons from pions and protons in the hadron arm and to distinguish the electrons from $\pi^{-}$in the electron arm. The method employed for the determination of the kaon yield is based on applying cuts on the corrected coincidence time, cuts on the Čerenkov detectors (Aerogel Čerenkov detectors in the hadron arm and Gas Čerenkov detector in the electron arm), acceptance cuts (through the R-function), vertex cuts, and momentum cuts.

In the corrected coincidence time spectra, (see Figure 55), the $K, \pi$ and proton peaks were identified and fit through a combination of three gaussian plus a polynomial (the polynomial function takes care of fitting the background in the corrected coincidence time spectra, TC_COR). The centroids of the three coincidence time peaks, corresponding to $e \pi, e p$ and $e K$ reactions, were extracted from the results of the fit.

The TC_COR cut (see Fig. 57) identifies the coincident kaons through their time of flight through the spectrometers and separates them from unwanted coincident pions and protons. The $\pi$ contamination is eliminated by optimizing the aerogel cuts. The coincidence time regions (for kaons protons and pions) were fitted with gaussians and the mean and the standard deviation were extracted from the fitting procedure. The standard deviation was used to correct for the TC_COR cut applied for each kinematic setting using the formula:

$$
T C_{-} C O R_{\text {Factor }}=\left[2 \cdot\left(\text { NORMDIST } \frac{3}{\sigma}-1\right)\right]^{-1}
$$




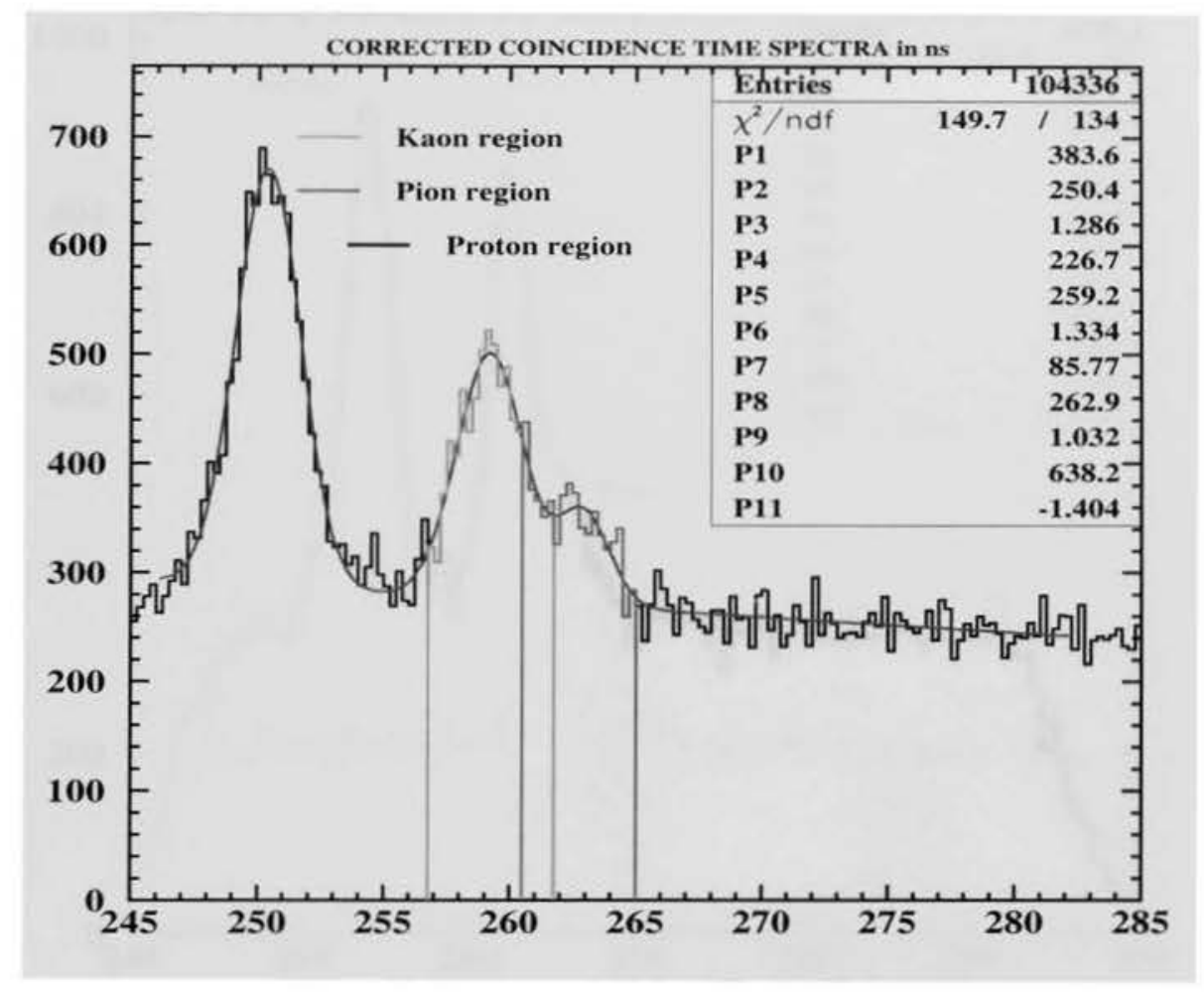

Figure 55: Corrected coincidence time spectra, identifying peaks after $A 1$ and $A 2$ cuts; Aerogel cuts are not optimized; $x$ axis is in ns.

where NORMDIST is the standard normal cumulative distribution function [GNU03], $\sigma$ is the standard deviation as extracted from the gaussian fit and $T C \_C O R_{\text {Factor }}$ is the coincidence time correction factor. The correction factors for the coincidence time cuts are shown in Tables 10 to 13 in Appendix A, for each kinematic setting. The coincident pions that are located underneath the $K$ peak, see Fig. 58, can be calculated assuming that the counts in the coincidence time spectra are Gaussian distributed. For this experiment the missing mass spectra was 


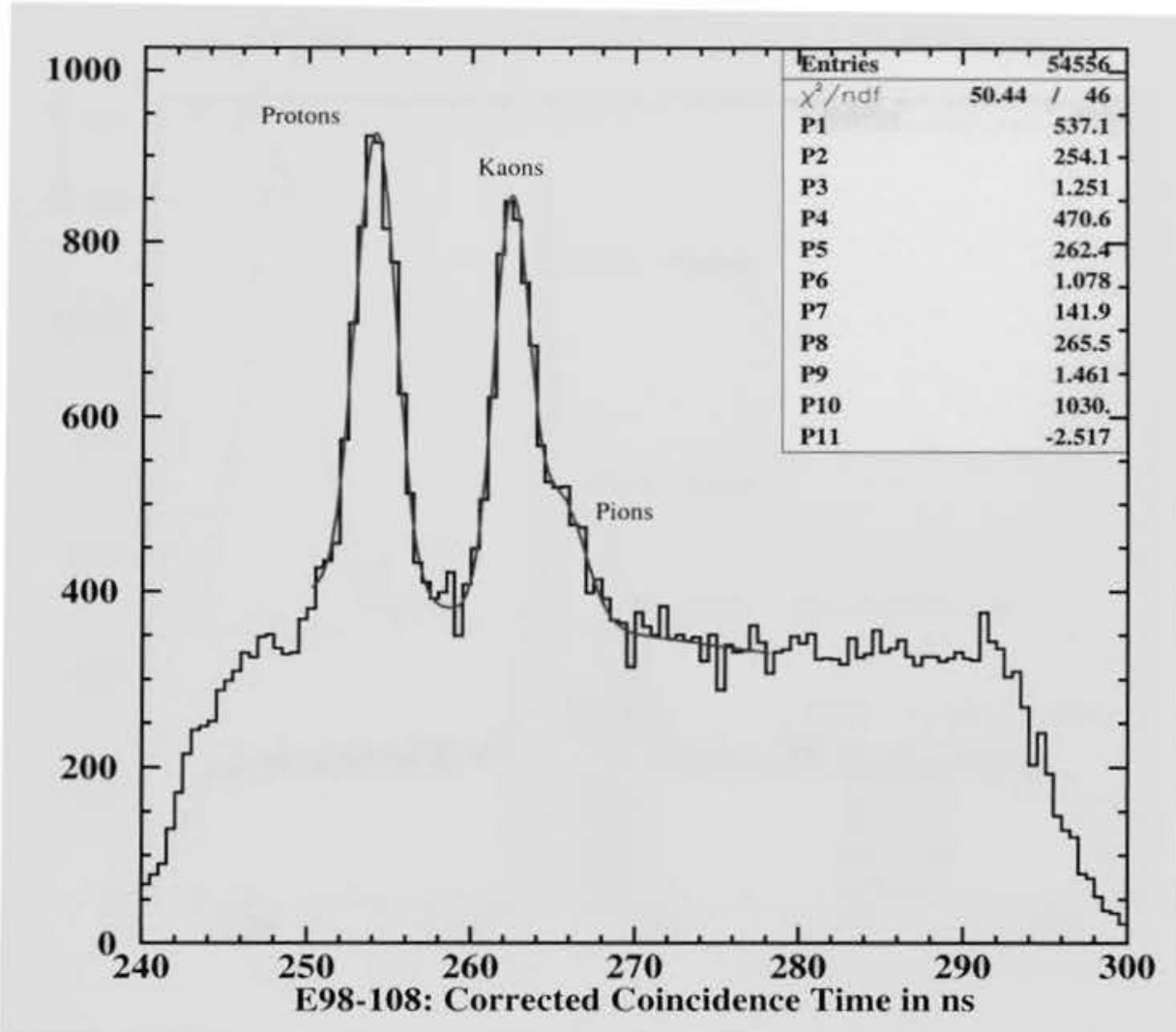

Figure 56: Fitted Corrected coincidence time spectra; proton, $K$ and $\pi$ peaks after $A 1$ and $A 2$ cuts; $\mathrm{x}$ axis is in ns.

obtained by adding software cuts on the aerogel detectors to the coincidence time cuts mentioned earlier. The aerogel cuts reduce to a minimum the coincident pions located under the kaon peak (Fig. 59 is before applying the aerogels cut and Fig. 60 is after applying the aerogels cut).

The left over coincident pions are corrected for by calculating the aerogel detectors' efficiencies (see Sections 3.6 and 3.7). The aerogel cut used in the analysis 


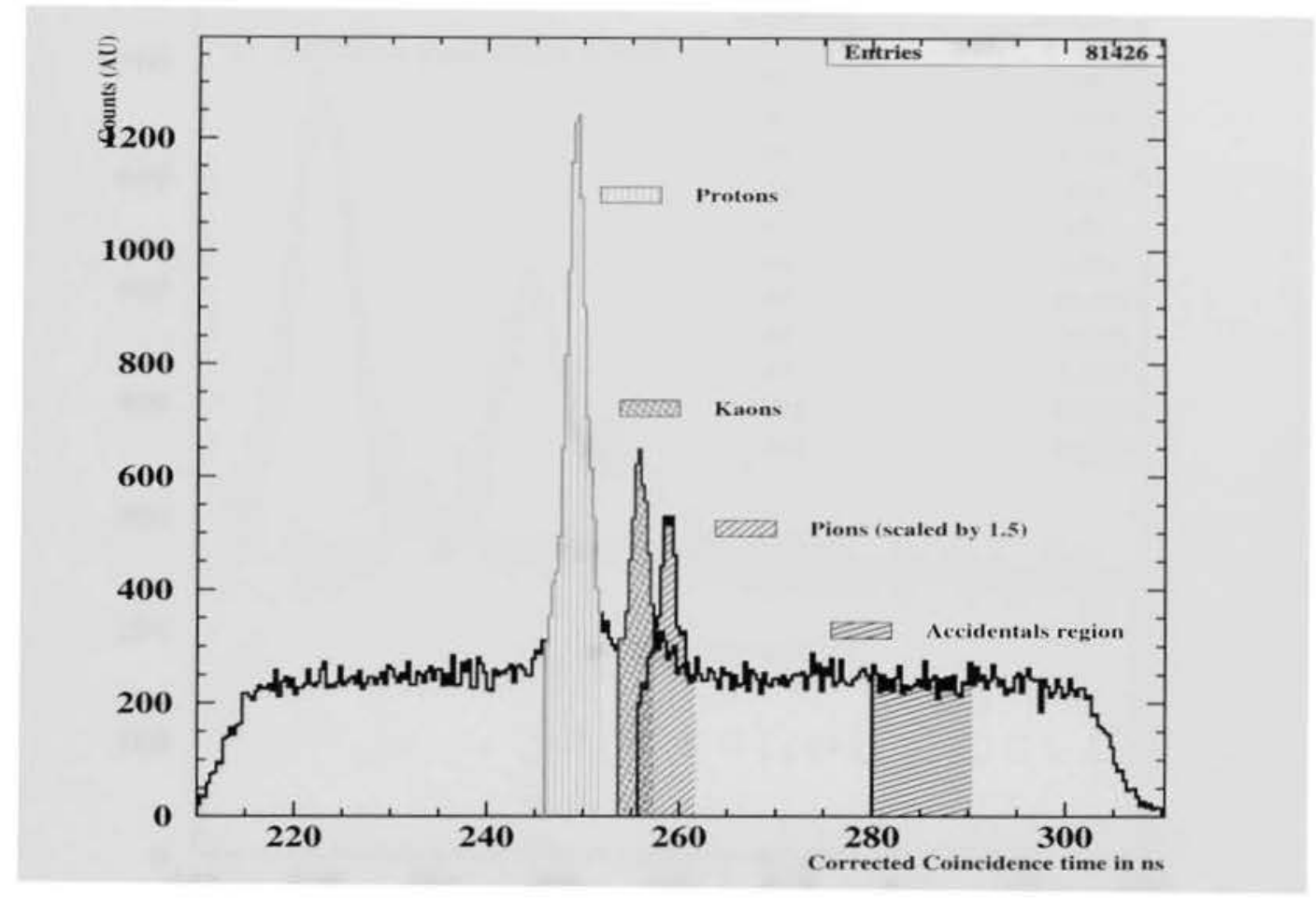

Figure 57: Coincidence time spectra, proton, $K, \pi$ and accidental regions; $\pi$ peak is scaled by $1.5 ; x$ axis is in ns.

consist of a logical $A N D$ condition between the number of photoelectrons $(P E)$ generated in $A 2$ and the negation of the number of photoelectrons generated in $A 1$ :

$$
\text { Aerogels }_{\text {cut }}: A 2 \cdot(\overline{A 1})
$$

where $\overline{A 1}$ is the logical NOT operator $(\neg A 1)$ applied to $A 1$. The $\pi$ rejection was based on the assumption that all $\pi$ seen by $A 2$ should also be seen by $A 1$. To verify this assumption we have determined the number of photoelectrons produced in $A 1$ by $\pi$ and the number of $P E$ produced in $A 1$ by the pions that fired $A 2$. 


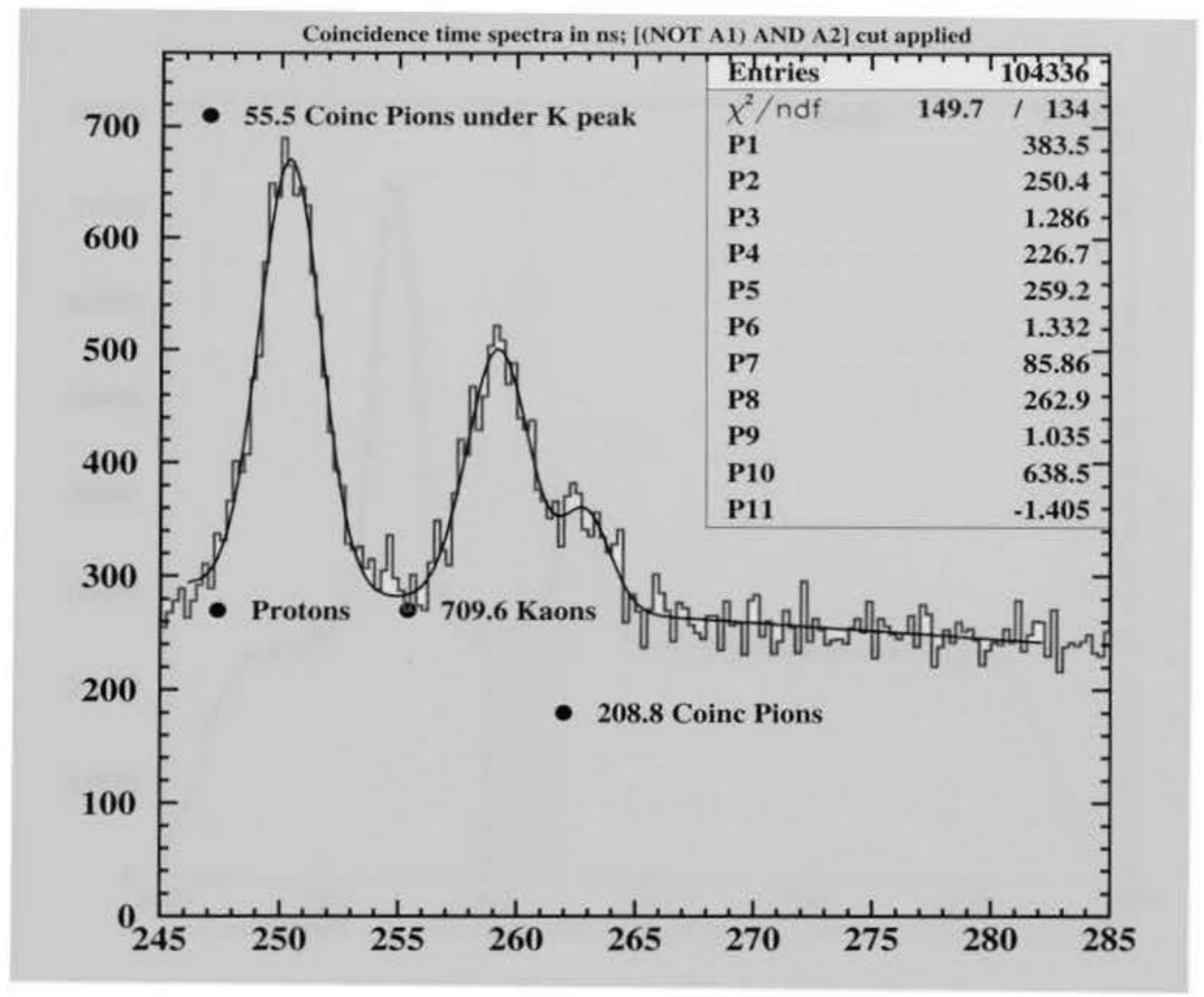

Figure 58: Yields from corrected coincidence time spectra.

Since there is no statistically significant difference in the spectra it means our assumption is correct (see Fig. 61).

During the analysis all particles that generated between $1.5 \mathrm{PE}$ and $50 \mathrm{PE}$ in $A 1$ were rejected (the $50 P E$ limit ensures that we include all pions that fired $A 1)$. Since $A 1$ fires only for $\pi$, we rejected all pions that fired $A 1$ and generated the specified number of $P E$. The second aerogel detector, $n=1.055$, fires on both kaons and pions (in a momenta range from $\approx 800 \mathrm{MeV} / \mathrm{c}$ to $\approx 2800 \mathrm{MeV} / \mathrm{c}$ ) so by applying a logical condition $A 2 \cdot(\overline{A 1}), A 2$ AND NOT $(A 1)$, we rejected the pions that fired both aerogel detectors while accepting the kaons that fired only $A 2$. The 


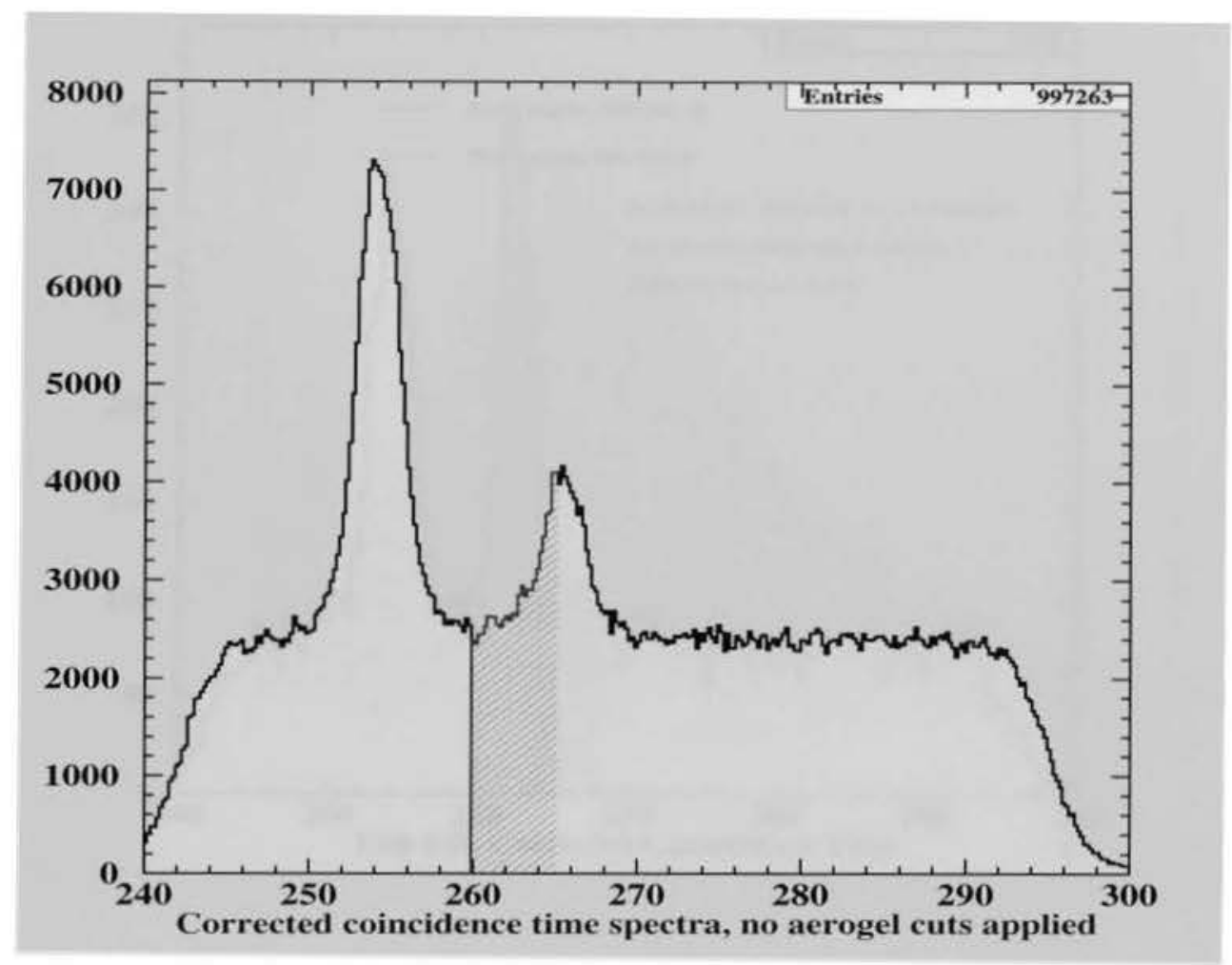

Figure 59: Corrected coincidence time spectra, before applying the aerogel cuts $(x$ axis is in ns). Pions and kaons cannot be distiguished.

$A 2$ cut on the number of photoelectrons was usually between $2 P E$ and $30 P E$ for low momenta in the hadron arm and between $3 P E$ and $50 P E$ for high momenta in the hadron arm (the cut was optimized according to hadron momenta such that the $A 2$ correction factor is not too big but at the same time the number of pions excluded was maximized). After fitting the corrected coincidence time spectra for each kinematic setting and extracting the centroids of the kaon peak the aerogel cuts are applied (see the logical operator described in Eq. 39). The difference in the missing mass spectra can be clearly seen in the figures below. For Fig. 62 no cuts 


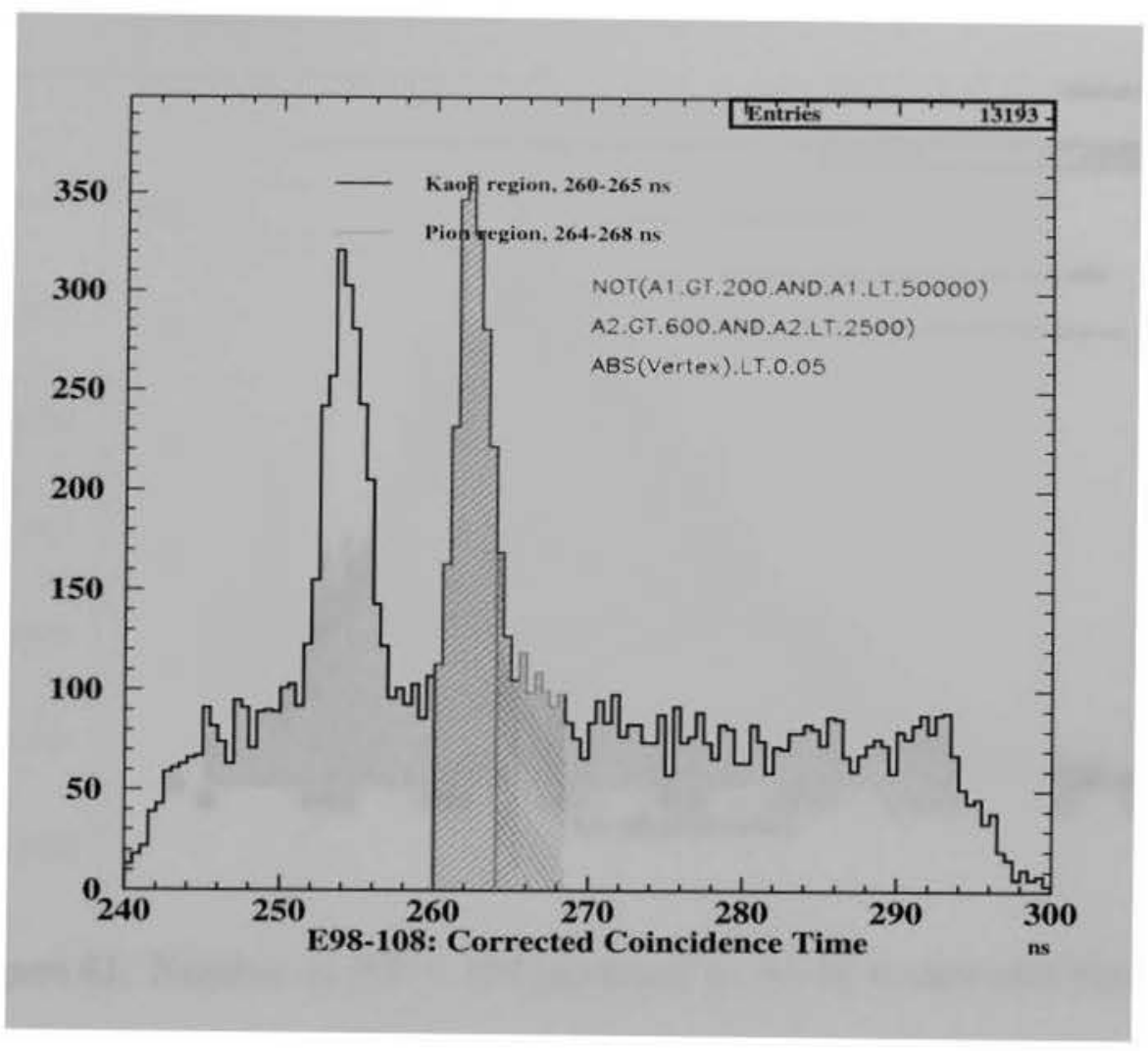

Figure 60: Corrected coincidence time spectra, after applying the aerogel cuts ( $x$ axis is in ns).

have been applied whereas in Fig. 63 only the coincidence time cut has been applied and in Fig. 64 and 65 the aerogel cuts have been added. Over the 2002 running

\begin{tabular}{|c|c|c|c|c|c|}
\hline Kin & $E^{\prime}$ & $E_{0}$ & $Q^{2}$ & $\theta_{E}$ & $W$ \\
\hline 1 & 3.077 & 5.6144 & 2.3275 & 21.1505 & 1.8205 \\
\hline 1 Prime & 3.145 & 5.7544 & 2.2813 & 20.451 & 1.8697 \\
\hline
\end{tabular}

Table 5: Kin 1 (2001) and Kin 1 Prime (2002); calculated $Q^{2}$ and $W$ values. 


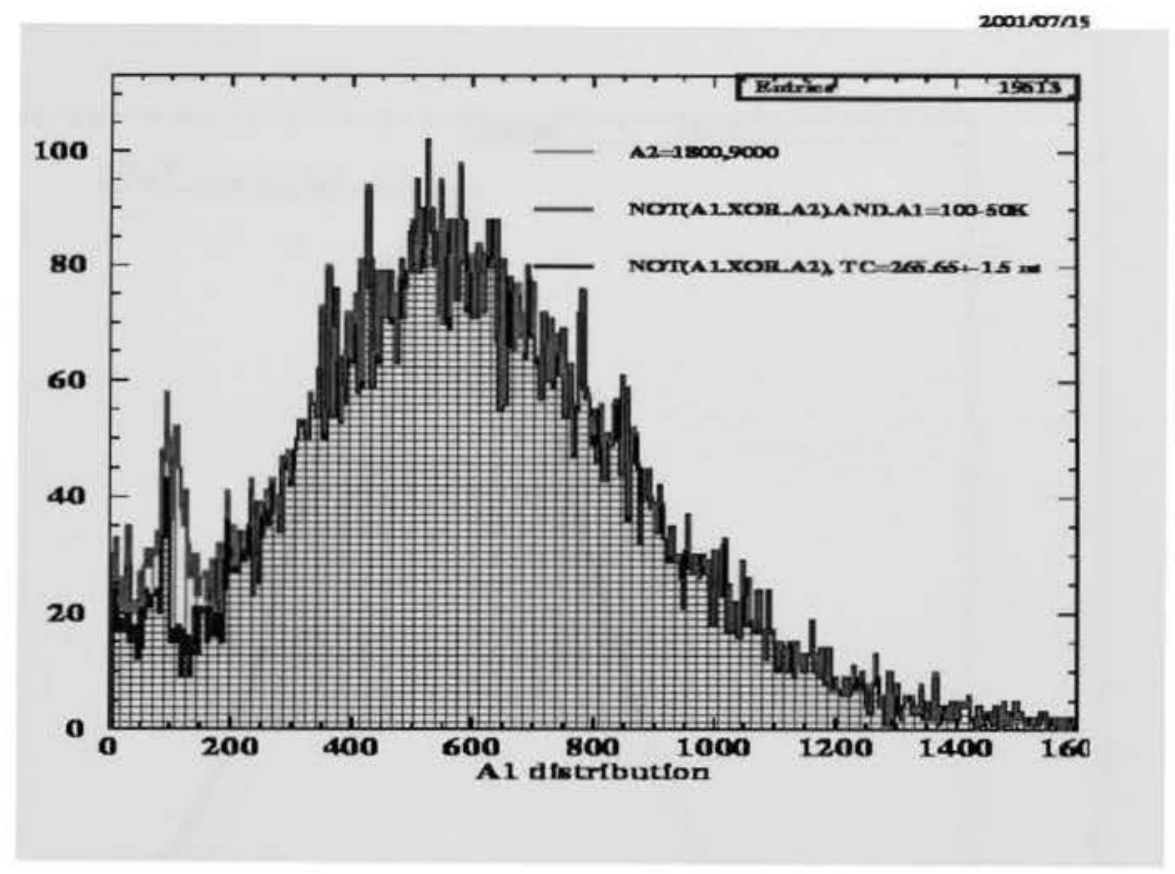

Figure 61: Number of $P E \times 100$ produced in $A 1$ by $\pi$ that also fired $A 2$.

period we acquired data for Kin 1 Prime (see Fig. 107), which had almost the same parameters as Kin 1 (data taken in 2001) see table 5.

In Table 5 the invariant mass, $W$, is calculated according to the formula:

$$
W=\sqrt{\left[M_{p}+\left(E_{0}-E^{\prime}\right)\right]^{2}-\left[Q^{2}+\left(E_{0}-E^{\prime}\right)^{2}\right]} .
$$

Using the cross sections determined for both kinematics, Kin1 and Kin 1 Prime, we extracted the longitudinal and transverse cross sections (see Fig. 136). The longitudinal and transverse response functions differ from those extracted without using kin 1 Prime (see Fig. 131). The percent difference in $W$ (for the central bin) is $3.31 \%$ whereas for $Q^{2}$ is $1.96 \%$. 


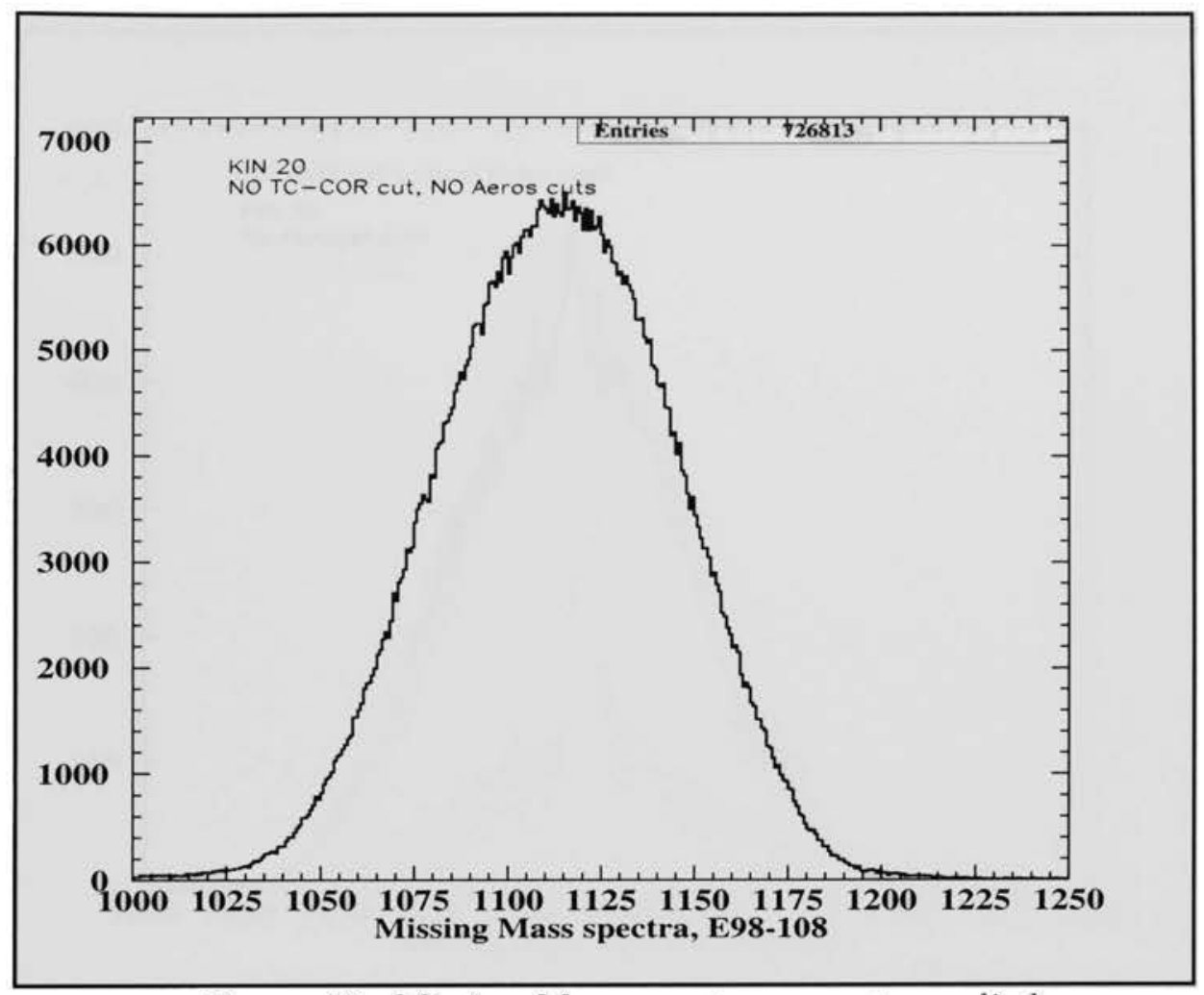

Figure 62: Missing Mass spectra, no cuts applied.

\subsection{Background subtraction}

In order to extract the contribution of the accidentals underneath the missing mass spectra two methods have been developed. The first method consists of fitting the background with a Gaussian function, as seen in Figs. 66 and 67 for two different kinematics, and then subtracting the fitting function from the main missing mass spectra (assuming the fitting function reproduces the background). Basically we subtracted a well behaved (smooth) function from the missing mass histogram. This method has the advantage that it subtracts a well behaved function from a histogram without suffering from the histogram's statistical fluctuations. The second method 


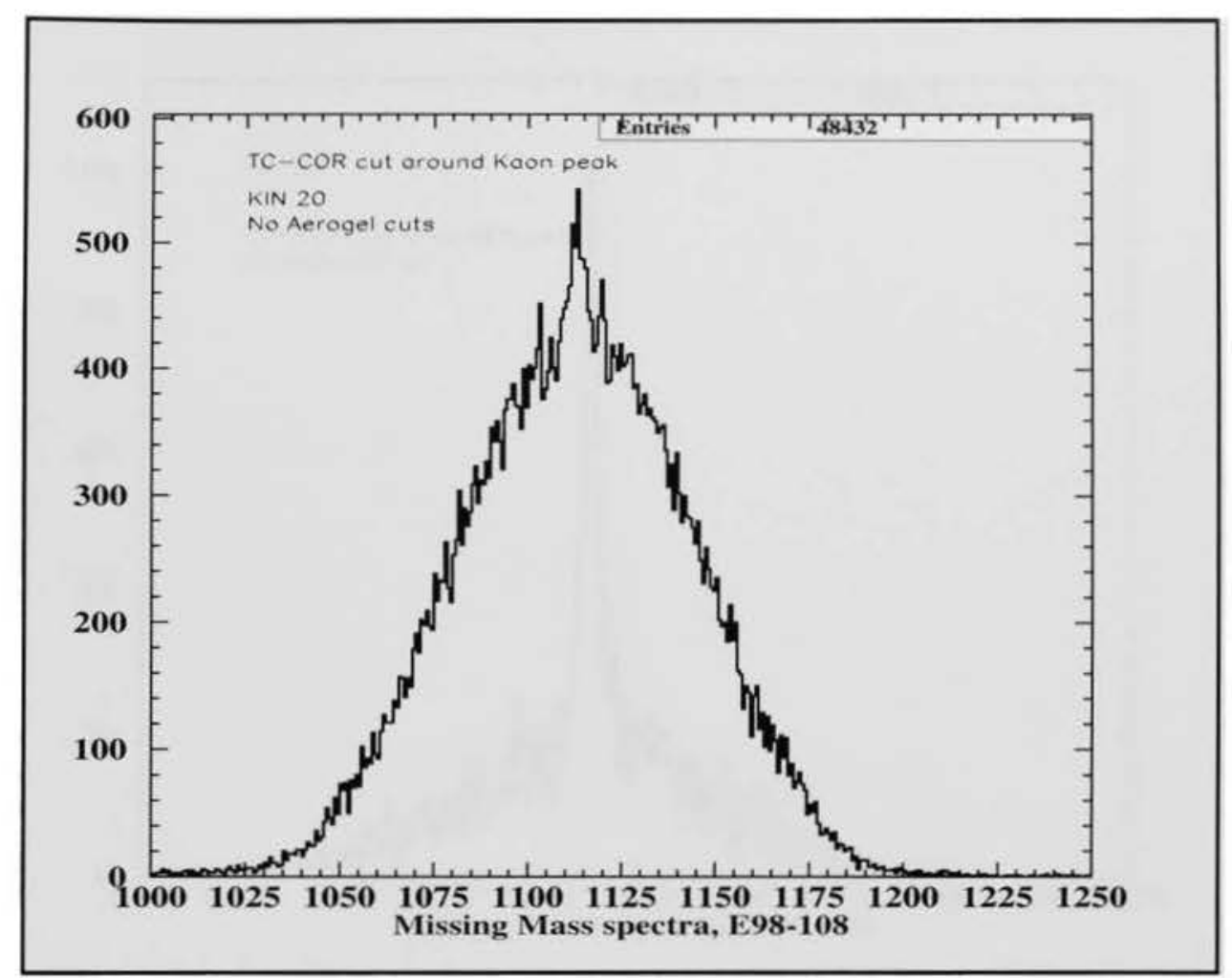

Figure 63: Missing mass spectra, with only coincidence time cut applied.

for background subtraction, consists of selecting a flat region (always 10 ns wide) in the corrected coincidence time spectra. The systematic error in subtracting the background (see Table 9) was estimated from the percent differences in the cross sections determined by using the yield as extracted with the two (background subtraction) methods described in this Section. The accidental region in the corrected coincidence time spectra (flat region) is used for producing the accidentals (for both $\Lambda$ and $\Sigma$ hyperons) under the missing mass spectra. Since the kaon region in the corrected coincidence time spectra is always 3 ns wide, while the flat region used 


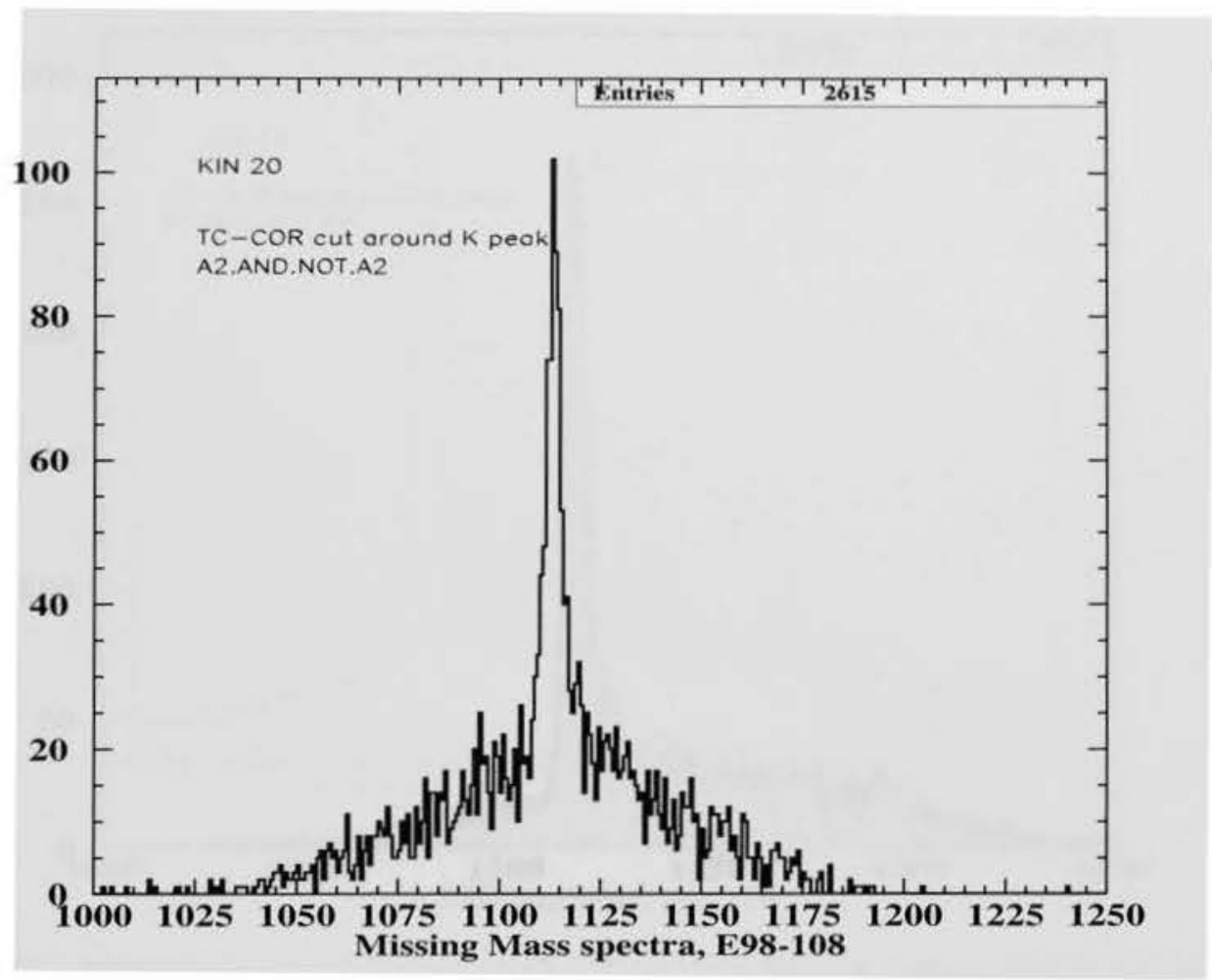

Figure 64: Missing mass spectra, with TC and aerogels cuts applied, Kin 20 ( $x$ axis is in $\left.\mathrm{MeV} / \mathrm{c}^{2}\right)$.

for accidentals is always $10 \mathrm{~ns}$, a scaling factor of 0.3 is used when subtracting the accidentals from the missing mass spectra:

$$
M M_{\Lambda, \Sigma}=M M_{\Lambda, \Sigma+A c c}-\left(0.3 \cdot M M_{A C C}\right)
$$

where $M M_{\Lambda, \Sigma}$ is the missing mass spectra for the two hyperons, $M M_{\Lambda, \Sigma+A c c}$ is the missing mass spectra that contains the accidentals and $M M_{A C C}$ is the missing mass spectra for accidentals only. 


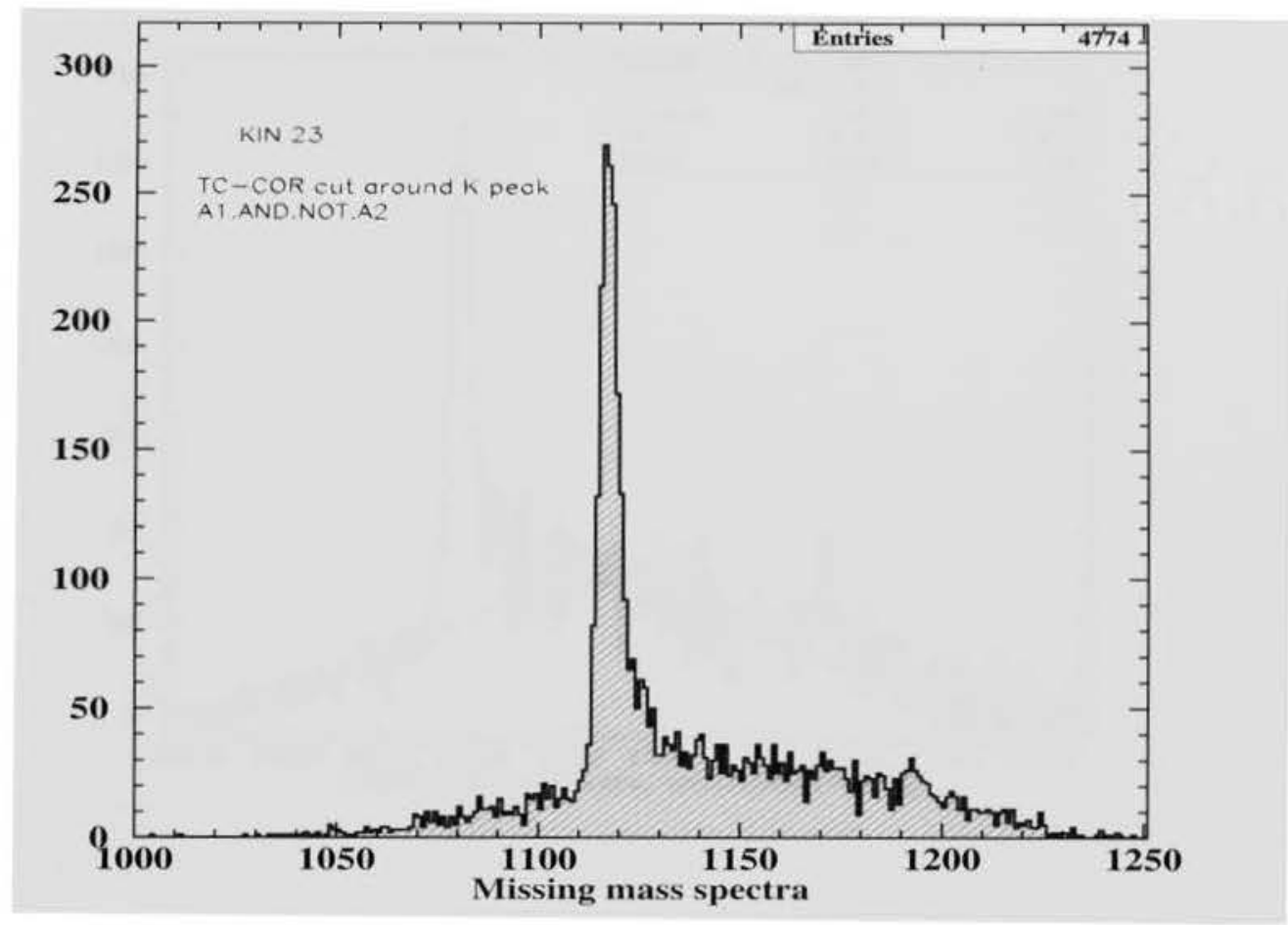

Figure 65: Missing mass spectra, with TC and aerogels cuts applied ( $x$ axis is in $\left.\mathrm{MeV} / \mathrm{c}^{2}\right)$, Kin 23.

The missing mass spectra with accidentals only (after scaling) is displayed in figures 69,70 and 71 for different kinematics. After subtracting the accidentals the associated statistical error was calculated by using the formula:

$$
\sigma_{Y i e l d}=\sqrt{\left[\left(0.3 \cdot \text { Sum }_{b c k g}\right)^{2}+\left(\text { Sum }_{k+a c c}\right)^{2}\right]}
$$

where $\sigma_{Y \text { ield }}$ is the absolute error in determining the clean kaon yield (without accidentals), Sum stacc $_{\text {is }}$ the yield of the kaons with background underneath $($ kaons + accidentals $)$ and $S u m_{b c k g}$ represents the background yield. The statistical errors associated with the background subtraction described above are specified for 


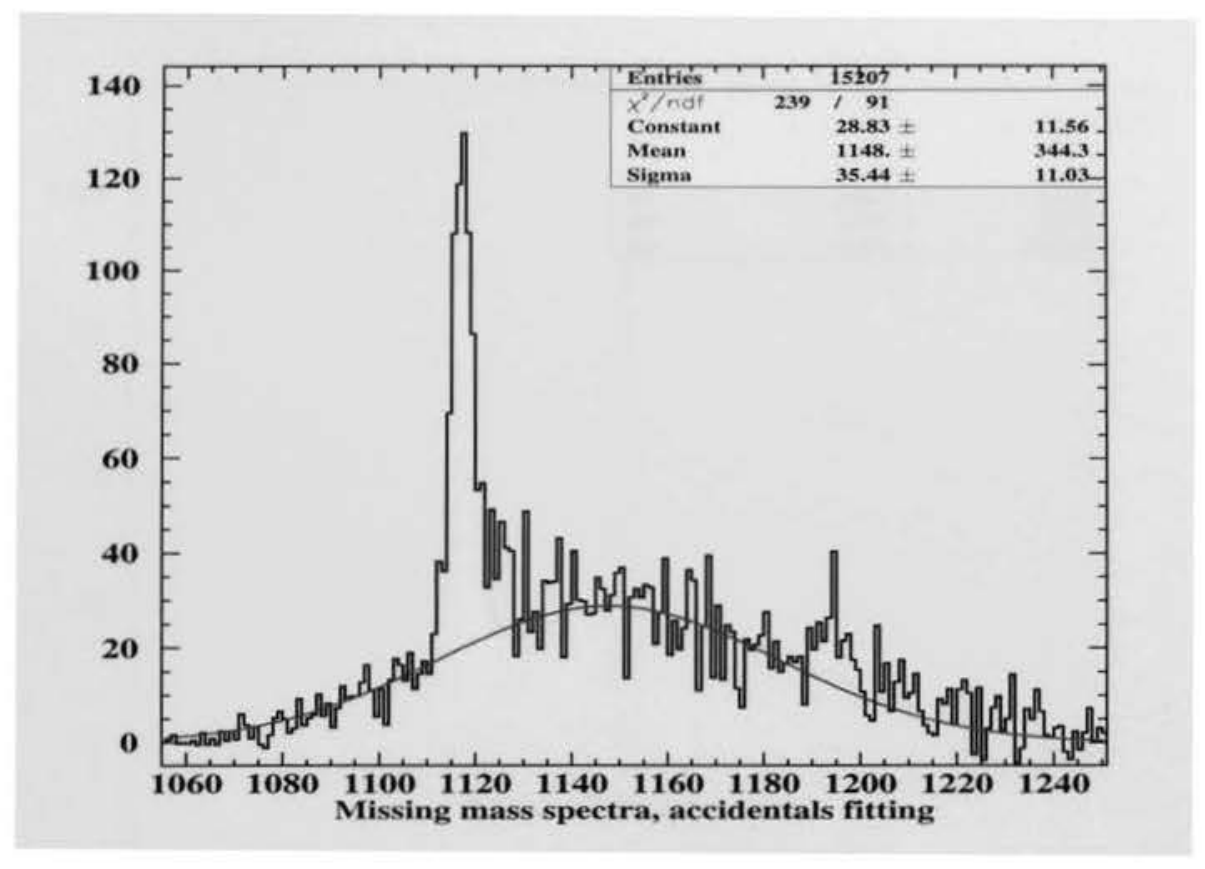

Figure 66: Missing mass spectra, accidentals fitting ( $x$ axis is in $\mathrm{MeV} / \mathrm{c}^{2}$ ).

each of the kinematics in the Table 6. Typical resolution (FWHM) obtained for $\Lambda$ and $\Sigma$ was $\approx 2.4 \mathrm{MeV} / \mathrm{c}^{2}$. 


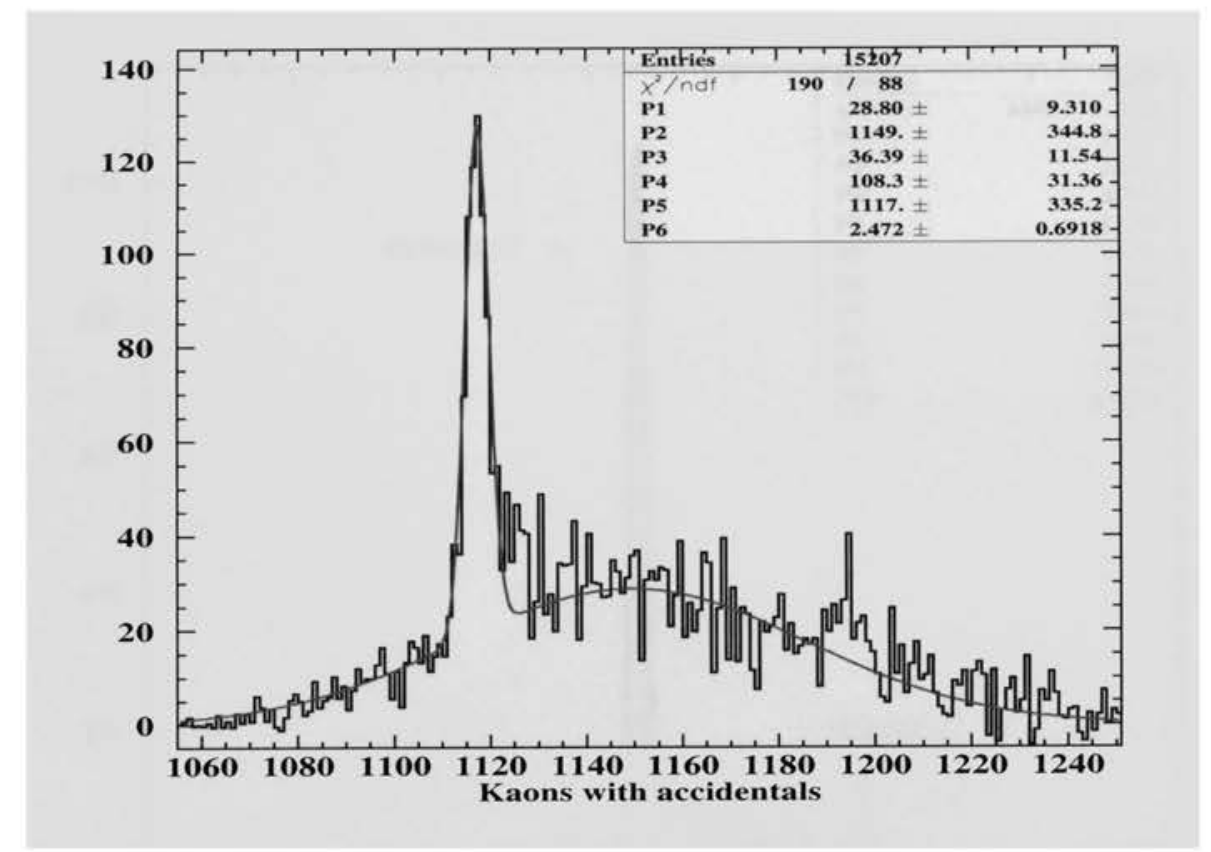

Figure 67: Missing mass spectra with accidental and $\Lambda$ fits $\left(x\right.$ axis is in $\left.\mathrm{MeV} / \mathrm{c}^{2}\right)$.

\subsection{Determination of spectrometer acceptance using the R-}

\section{function}

By definition, the sign of an R-function is determined by the signs of its arguments. The value of the R-function [RVA00] is equal to 0 on the boundary, less than 0 outside and greater than 0 inside the region. The determination of the spectrometer acceptance was done by calculating on an event-by-event basis the R-function. In order to select a flat region region of the spectrometers' acceptance, we used the Rfunction (flat - a region where the acceptance function is constant. The R-function 


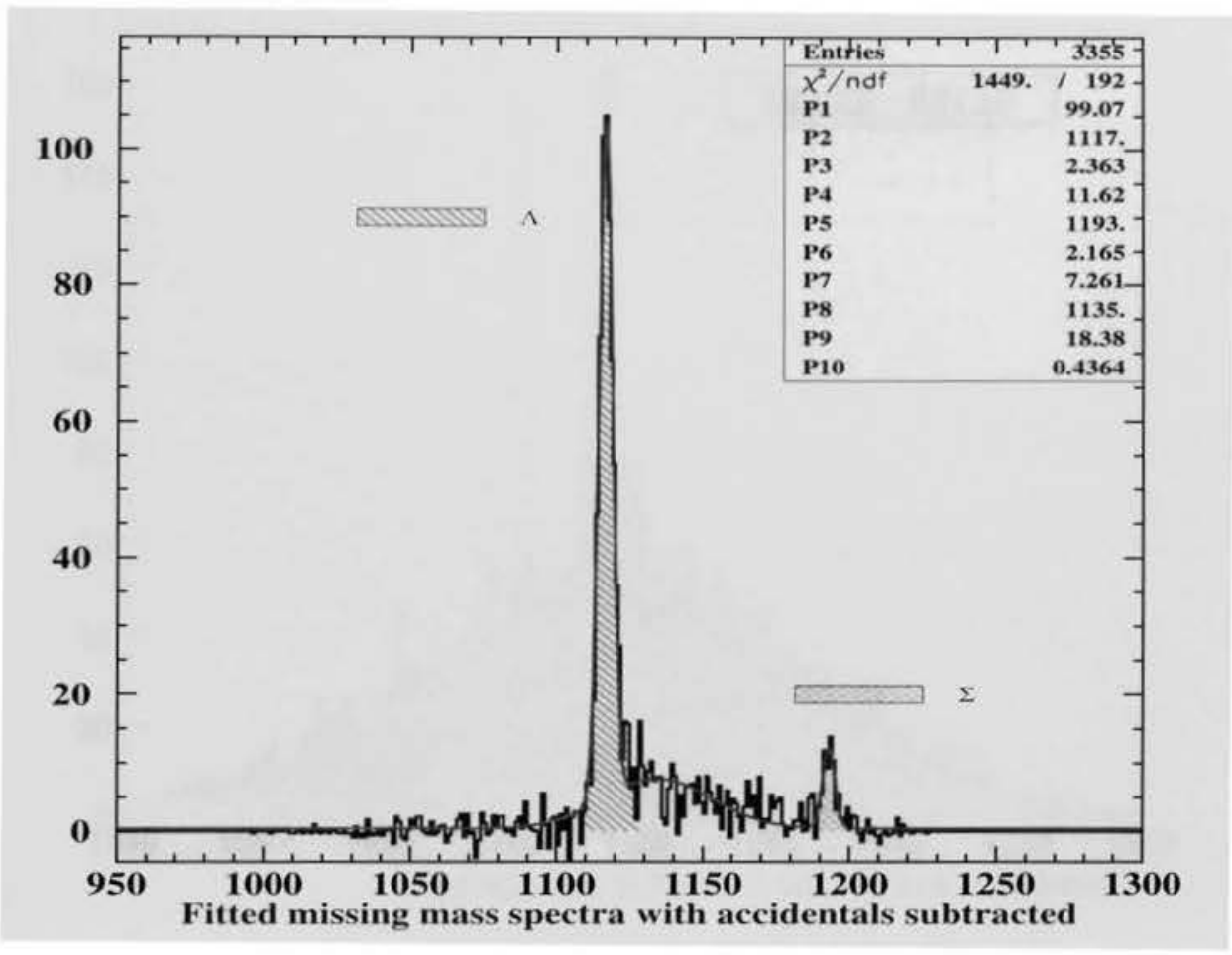

Figure 68: Fitted missing mass spectra with accidentals subtracted, for Kin 3 ( $x$ axis is in $\left.\mathrm{MeV} / \mathrm{c}^{2}\right)$.

uses as input a model of the acceptance boundary of each spectrometer and evaluates the location of particles' trajectory with respect to this boundary.

The trajectory of the particles is determined by five variables in each spectrometer: two angles in the dispersive $(\theta)$ and non-dispersive planes $(\phi)$, the dispersive position at the scattering point in the target $\left(x_{t g}\right)$, the non-dispersive position at the scattering point in the target $\left(y_{t g}\right)$ and the relative momenta, $\delta p=\left(p-p_{0}\right) / p_{0}$, where $p_{0}$ is the central momentum of the spectrometer and $p$ is the particle's momentum. 


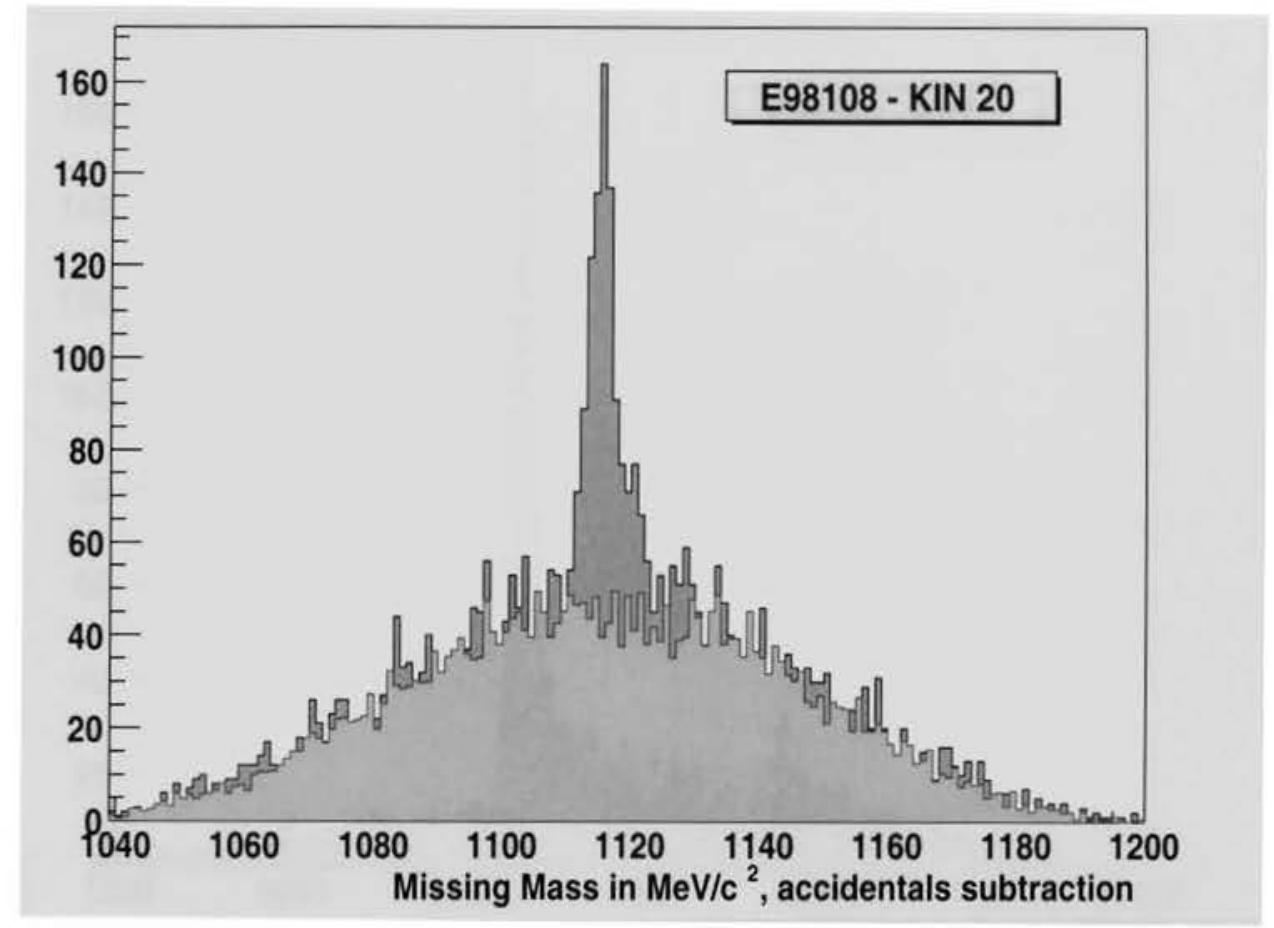

Figure 69: Missing mass spectra with accidental subtraction for Kin 20.

The advantage of using $\mathrm{R}$-function is that it reproduces well the optimal region of the acceptance of the electron and hadron spectrometers and it has been verified and used for previous experiments. The disadvantage of using it is that it reduces the yield in the $\Lambda$ spectra by about $75 \%$, resulting in an increase of the statistical error from $\approx 2.5 \%$ to $\approx 5.2 \%$ (see Figs. 72 and 73 ). Another effect of using the R-function is that the $\Sigma$ hyperon missing mass is obtained with very poor statistics for most kinematics so no cross sections were extracted for $\Sigma$ (see Section 4.3). The phase space coverage for the $\Sigma$ was smaller than for the $\Lambda$ (see Appendix 5.1), already contributing to a smaller statistics for the $\Sigma$ yield. 


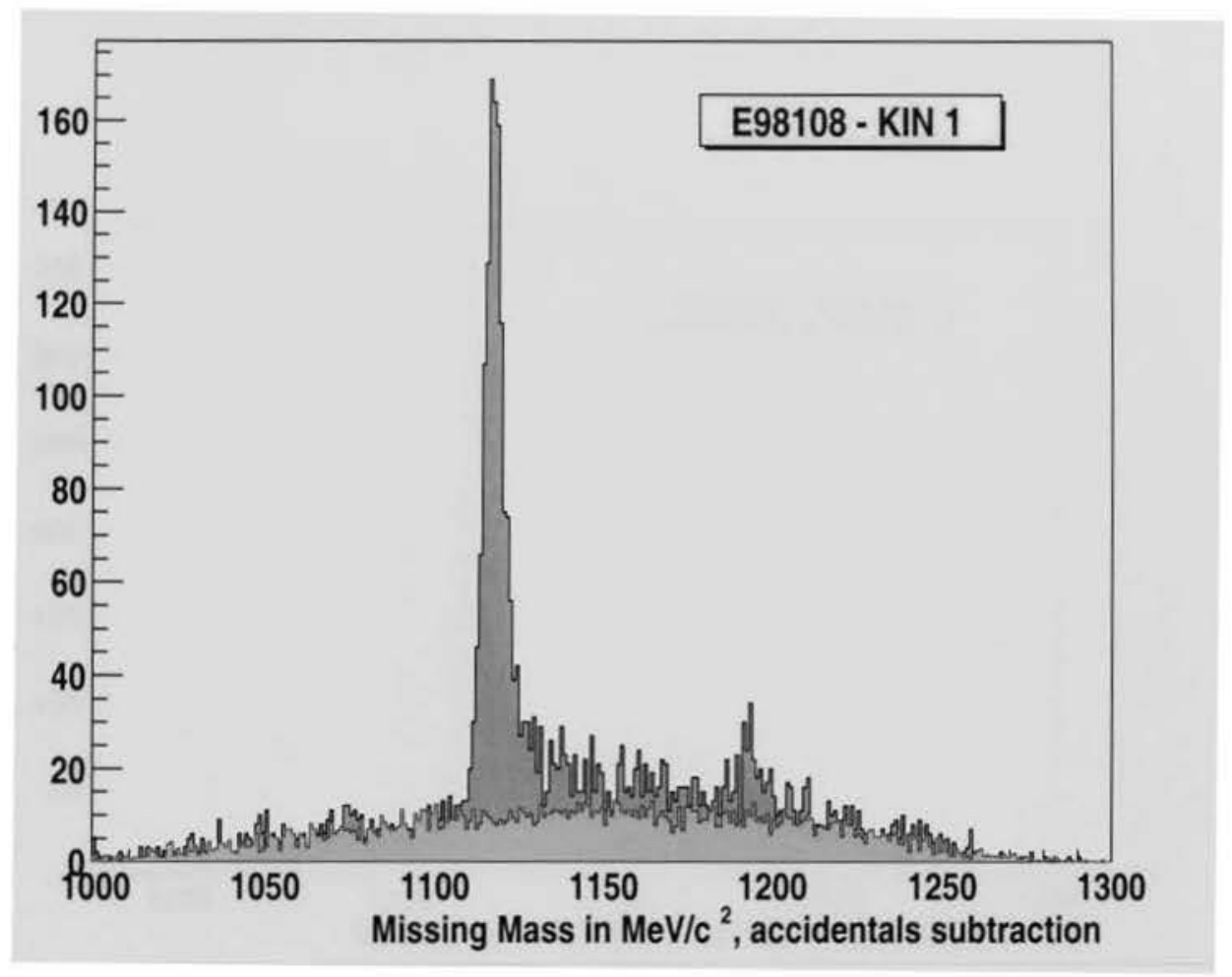

Figure 70: Missing mass spectra with accidental subtraction for Kin 1.

By applying a cut on the R-function one can include only the trajectories that are inside the nominal acceptance of the spectrometers [LER02]. The R-function cut for all kinematics in this experiment, was greater than $0.008, R F N_{E, H}>0.008$, meaning all trajectories that lie $8 \mathrm{mrad}$ or more inside the solid angle defined for a specified value of $y_{t g}$ and $\delta p$ were accepted (by definition the R-function returns a value equal to the distance, in units of radians, of the input trajectory from the boundary of the nominal acceptance [RVA00]). Here $R F N_{E, H}$ is the R-function cut in the electron or hadron arm. Thus the R-function provides a mean to impose a 


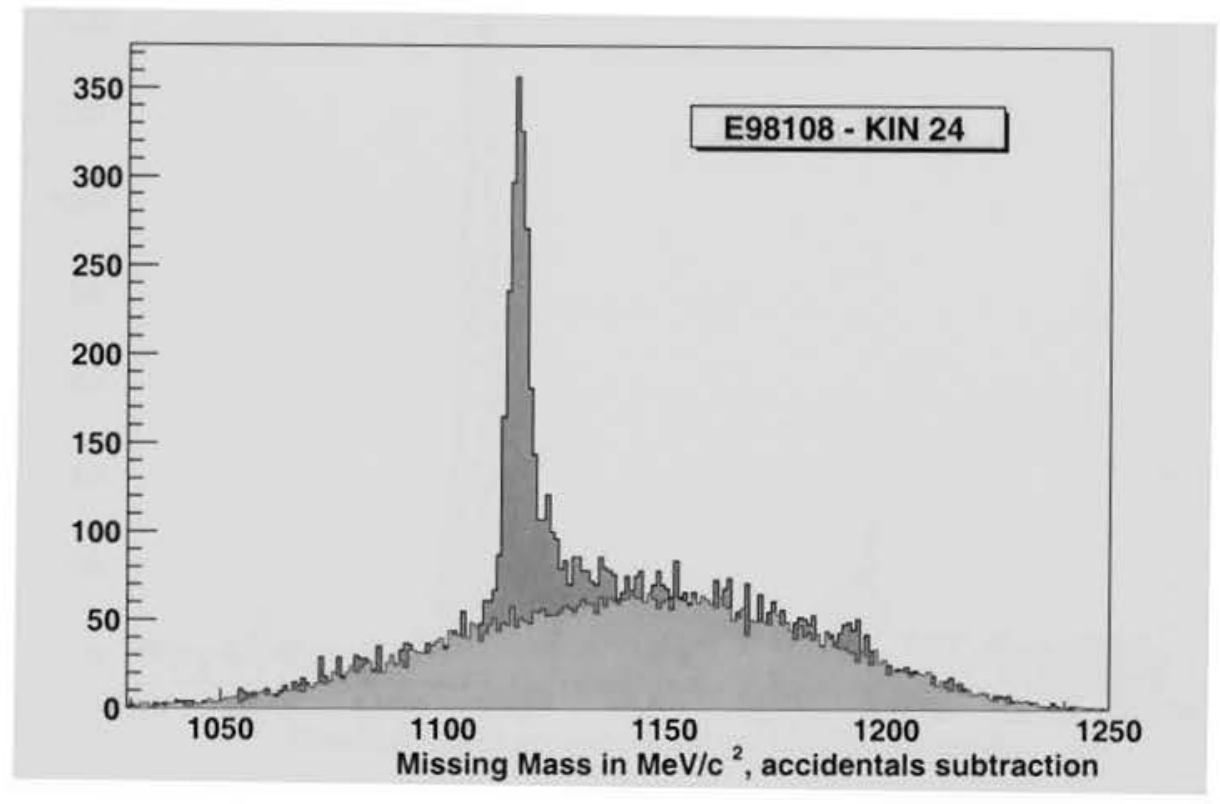

Figure 71: Missing mass spectra with accidental subtraction for Kin 24 .

multi-dimensional boundary of the HRS solid angles. The same R-function cut was applied in the Monte Carlo simulation of the kaon electroproduction (see Section 4.4).

\subsection{Monte Carlo simulation}

A complete and accurate Monte Carlo simulation is required in order to be able to extract cross sections. The MCEEP [ULM02] simulation program written by Paul Ulmer was used to simulate the $\left(e, e^{\prime} K\right)$ reaction [MAR02]. It is based on the general formalism of exclusive electron-nucleon $\left(e, e^{\prime} N\right)$ reaction cross section developed by 


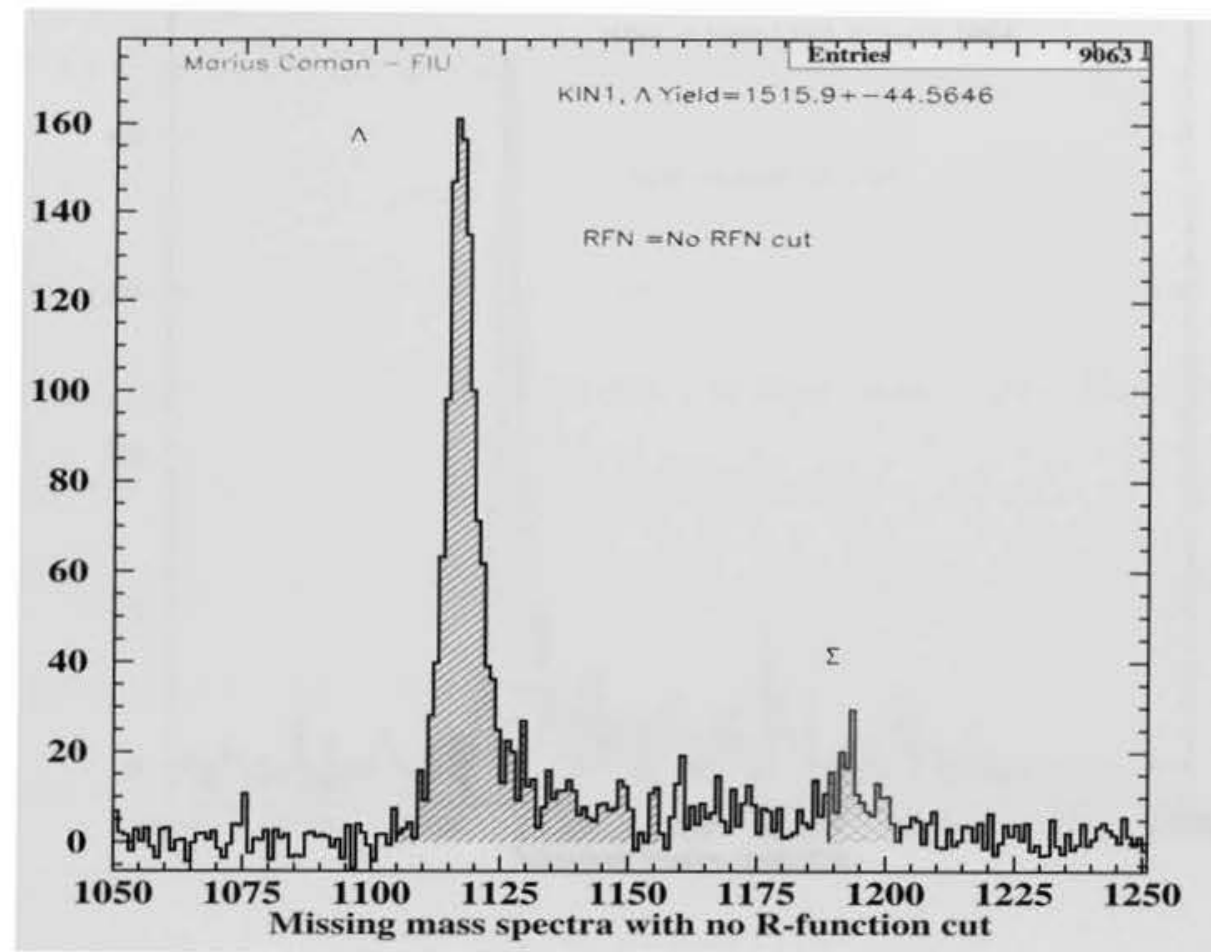

Figure 72: Missing mass spectra with no R-function cut, Kin $1\left(x\right.$ axis is in $\left.\mathrm{MeV} / \mathrm{c}^{2}\right)$.

Picklesimer and Van Orden [PIC89]. Monte Carlo for $\left(e, e^{\prime} p\right)$ reactions (MCEEP) is designed to simulate coincidence $\left(e, e^{\prime} X\right)$ experiments by averaging theoretical models over an experimental acceptance [ULM02]. Detector acceptances, Fermi motion, radiative corrections, physics weighting, and absolute normalizations for the simulated events are modeled in order to obtain valid results.

MCEEP allows for a general 3-D beam target interaction region thus one can evaluate the effects of extended targets (a $15 \mathrm{~cm} \mathrm{LH}_{2}$ extended target was used during the E98-108 experiment) and rastered beams. MCEEP simulates also radiative 


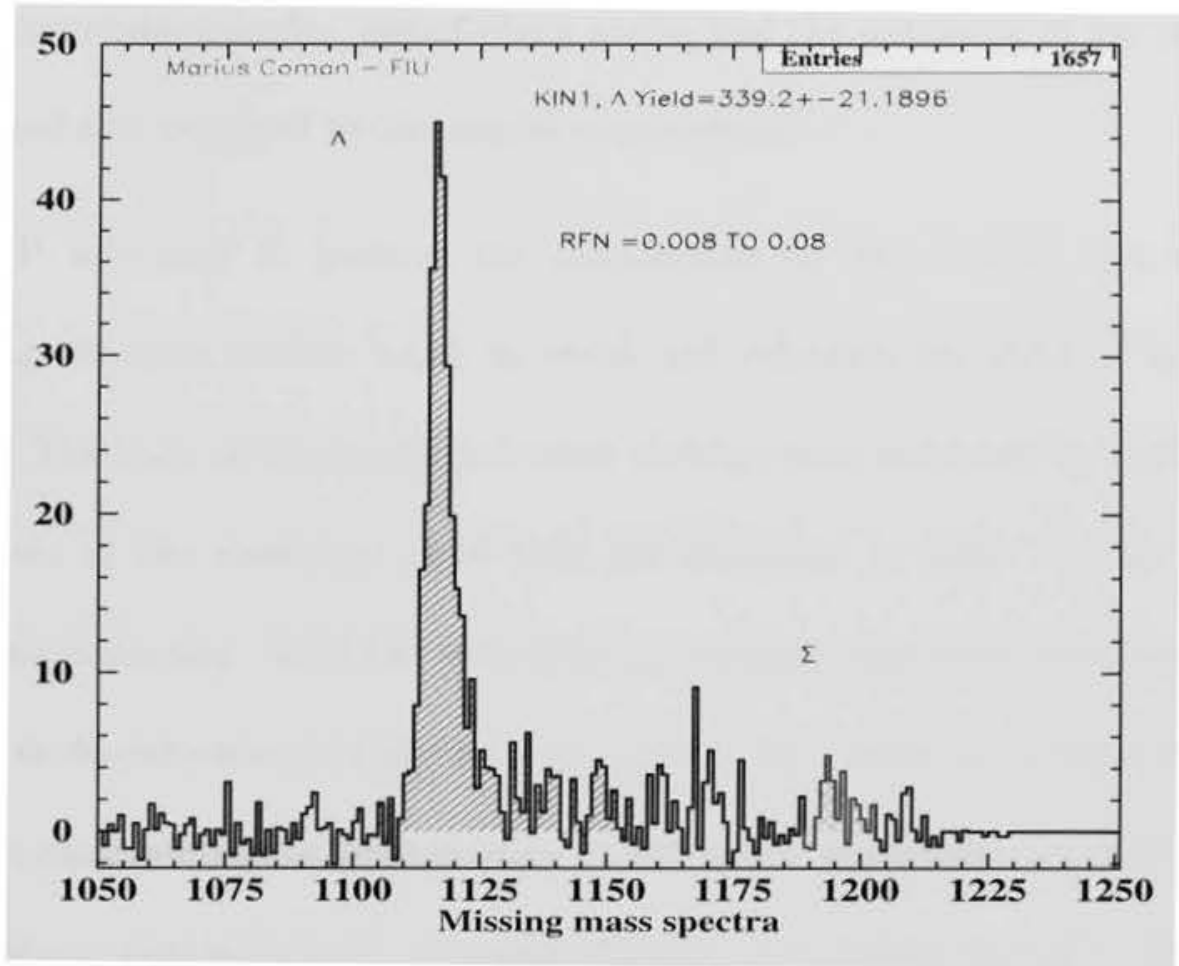

Figure 73: Missing Mass spectra, with R-function cut applied, Kin 1 ( $x$ axis is in $\left.\mathrm{MeV} / \mathrm{c}^{2}\right)$.

effects and realistic multiple scattering. MCEEP handles both internal and external radiative corrections by the electron.

The events are randomly generated within the spectrometer experimental acceptance. An event is defined as any combination of variables which fully specifies the reaction in the laboratory frame. Each event is characterized by choosing for the emerging particle a randomly selected momentum and two orientation angles. This characterization of the particles is done for both electrons and hadrons. For a single event, one ends up with six coordinates for the scattered electron and the emerging 
hadron. The in-plane angles, out-of-plane angles and the momenta of the particles are generated and weighted by the model cross-section.

MCEEP was used to perform the calculations for the $\left(e, e^{\prime} K\right)$ reaction, by determining the cross section event by event and calculate the yield of kaons for this event. The kaon electroproduction cross sections were extracted by performing a comparison of the simulated yield with the measured $\Lambda$ yield. In the case of kaon electroproduction, MCEEP was used to calculate the yield considering the scattering to discrete states of the residual system, e.g., the $\Lambda$ or $\Sigma$ hyperons, and this implies constraining the missing mass. A cut on the mass of the residual system, $1105.0<M_{\Lambda}<1155.0 \mathrm{MeV} / \mathrm{c}^{2}$, was used (the $M_{\Lambda}=1115.683 \mathrm{MeV} / \mathrm{c}^{2}$ ). Thus the MCEEP yield is obtained integrating over a five-fold acceptance volume:

$$
Y i e l d^{M C E E P}=L \int_{\triangle V} \frac{d^{5} \sigma}{d E^{\prime} d \Omega_{e} d \Omega_{K}} d E^{\prime} d \Omega_{e} d \Omega_{K}
$$

where the $L$ represents the luminosity

$$
L=\frac{\rho \cdot \text { length }_{\text {target }} \cdot N_{A} \cdot Q}{e \cdot A}
$$

$\Delta V$ represents the phase space, $d E^{\prime}$ is the electron's final energy, $d \Omega_{e}$ and $\Omega_{K}$ are the electron and kaon solid angles respectively.

\subsubsection{Radiative corrections}

The $\left(e, e^{\prime} N\right)$ reaction does not only proceed through the simple, one photon exchange diagram. The incoming and the outgoing charged particles radiate real photons due 
to changes in their velocity by the Coulomb field of the nucleus involved in the scattering process. This is called internal bremsstrahlung, as opposed to external bremsstrahlung when the charged particles interact with a nucleus other than the one involved in the scattering process. The result of these interaction is the emission and reabsorption of virtual photons and the emission of real soft photons in which the energy of the emitted photons is very small compared with both the incident and the emergent electron energies.

The radiated photons change the cross section for the reaction and the apparent energy and the momentum transfer (loss of energy and change of the direction). Although these real physical processes have this two-fold effect on the data, usually they are not taken into account in most theoretical calculations because they are experiment specific. So these effects have to be unfolded from the experimental data in order to get the underlying physics. Figure 74 represents a comparison between the missing mass as produced through the Monte Carlo simulation and the $\Lambda$ missing mass as obtained from data. For the E98-108 experiment, MCEEP has been used with the multi-photon correction turned on (a method in which the multi-photon contribution to the radiative tail is derived), which also includes the virtual photon part of the Schwinger correction (both real and virtual photons' contributions are accounted for) [ULM02]. The radiative corrections were only done for the electrons, not the other particles in the reaction.

The agreement between the spectra is good, the simulation showing a slight overestimate of the radiative tail. 


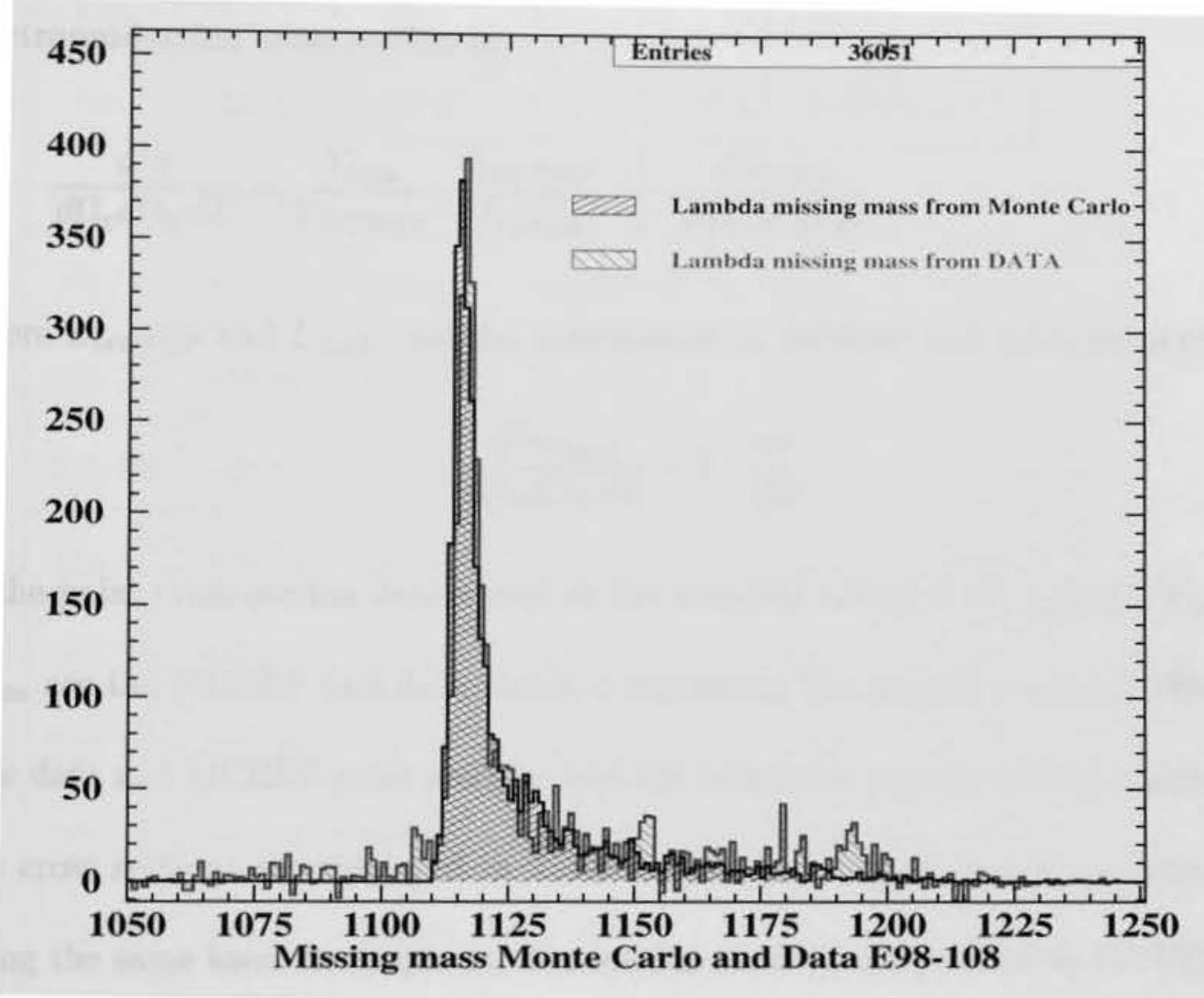

Figure 74: $\Lambda$ missing mass Monte Carlo simulation and data $\left(x\right.$ axis is in $\left.\mathrm{MeV} / \mathrm{c}^{2}\right)$.

\subsection{Cross section calculation}

We determined the kaon electroproduction cross section for the experiment by using the yields extracted from data (their calculation is described in detail in the previous sections) and the yield obtained from the Monte Carlo Simulation (by using MCEEP as described in section 4.4). In order to account for different luminosities in MCEEP and DATA, a correction factor (the ratio of the luminosity as determined from the data and the luminosity parameter used in the MCEEP simulation: $\left.\frac{L_{M C E E P}}{L_{D A T A}}\right)$ was 
introduced in the cross section formula. The formula used to calculate the kaon electroproduction cross section is:

$$
\frac{d^{5} \sigma}{d \Omega_{e} d \Omega_{K} d E}=\frac{Y_{\text {data }}}{Y_{M C E E P}} \cdot \frac{L_{M C E E P}}{L_{D A T A}} \cdot \frac{1}{\varepsilon} \cdot \frac{d^{5} \sigma_{P o i n t}}{d \Omega_{e} d \Omega_{K} d E}
$$

where $L_{M C E E P}$ and $L_{D A T A}$ are the luminosities in MCEEP and data respectively,

$$
\frac{d^{5} \sigma_{\text {Point }}}{d \Omega_{e} d \Omega_{K} d E}=\Gamma \cdot \frac{d \sigma}{d \Omega}
$$

is the point cross section determined at the nominal values of $W$ and $Q^{2}, Y_{M C E E P}$, $Y_{\text {data }}$ are the MCEEP and data yields, $\varepsilon$ represents the overall detectors' efficiency. The data and MCEEP cross sections and the correction factors used in determining the cross sections are displayed in Table 6 . The point cross section, is determined using the same kaon electroproduction routine that is incorporated in MCEEP. 


\begin{tabular}{|c|c|c|c|c|c|c|}
\hline $\begin{array}{l}\text { Kin. } \\
\text { No. }\end{array}$ & $\begin{array}{l}\frac{d \sigma}{d \omega}_{\Lambda}^{\text {Point }} \\
(\mathrm{nb} / \mathrm{sr})\end{array}$ & $\begin{array}{c}\frac{d \sigma}{d \Omega} \Lambda \\
(\mathrm{nb} / \mathrm{sr})\end{array}$ & $\begin{array}{l}\text { Corrected } \\
\text { Yield }(\mathrm{Hz})\end{array}$ & $\begin{array}{l}\text { Radiative } \\
\text { Corr. Factor }\end{array}$ & $\begin{array}{l}\text { Kaon } \\
\text { Yield }\end{array}$ & $\begin{array}{c}\text { Relative } \\
\text { Error }\end{array}$ \\
\hline Kin $1 \mathrm{P}$ & 149.42 & 130.11 & 0.0215 & 1.071 & 485.4 & 5.25 \\
\hline Kin 1 & 148.82 & 150.53 & 0.0309 & 1.041 & 339.1 & 6.45 \\
\hline Kin 23 & 138.88 & 134.71 & 0.0065 & 1.054 & 481.1 & 5.46 \\
\hline Kin 20 & 125.67 & 130.27 & 0.0016 & 1.084 & 159.8 & 8.74 \\
\hline Kin 2 & 151.84 & 150.08 & 0.0373 & 1.087 & 531.2 & 4.54 \\
\hline Kin 24 & 140.89 & 135.36 & 0.0078 & 1.044 & 546.6 & 4.69 \\
\hline Kin 18 & 126.45 & 129.13 & 0.0016 & 1.098 & 175.7 & 8.22 \\
\hline Kin 3 & 148.76 & 147.40 & 0.0562 & 1.100 & 914.7 & 3.94 \\
\hline Kin 25 & 135.65 & 135.13 & 0.0093 & 1.056 & 676.1 & 6.33 \\
\hline $\operatorname{Kin} 4$ & 141.32 & 137.15 & 0.0647 & 1.086 & 1539.4 & 2.95 \\
\hline Kin 22 & 126.80 & 118.13 & 0.0076 & 1.061 & 580.4 & 5.08 \\
\hline
\end{tabular}

Table 6: Unseparated cross sections and corresponding kaon yields for $\mathrm{Q}^{2}=2.35 \mathrm{GeV}^{2}$. Errors are statistical and include background subtraction and acceptance cuts. 


\begin{tabular}{|c|c|c|c|c|c|c|}
\hline $\begin{array}{l}\text { Kin. } \\
\text { No. }\end{array}$ & $\begin{array}{l}\frac{d \sigma}{d \omega} \Lambda^{\text {Point }} \\
(\mathrm{nb} / \mathrm{sr})\end{array}$ & $\begin{array}{c}\frac{d \sigma}{d \Omega} \Lambda \\
(\mathrm{nb} / \mathrm{sr})\end{array}$ & $\begin{array}{l}\text { Corrected } \\
\text { Yield }(\mathrm{Hz})\end{array}$ & $\begin{array}{l}\text { Radiative } \\
\text { Corr. Factor }\end{array}$ & $\begin{array}{l}\text { Kaon } \\
\text { Yield }\end{array}$ & $\begin{array}{c}\text { Relative } \\
\text { Error }\end{array}$ \\
\hline Kin 11 & 187.14 & 177.57 & 0.0756 & 1.090 & 842.1 & 4.86 \\
\hline Kin 26 & 174.82 & 170.70 & 0.0192 & 1.062 & 727.4 & 6.80 \\
\hline Kin 19 & 159.15 & 149.68 & 0.0038 & 1.121 & 455.5 & 7.67 \\
\hline Kin 5 & 185.18 & 178.85 & 0.0812 & 1.080 & 1413.6 & 3.29 \\
\hline Kin 27 & 172.93 & 169.96 & 0.0189 & 1.049 & 769.4 & 4.41 \\
\hline Kin 17 & 156.49 & 154.39 & 0.0037 & 1.091 & 376.7 & 4.53 \\
\hline Kin 12 & 182.10 & 171.78 & 0.0939 & 1.084 & 1198.4 & 4.13 \\
\hline Kin 28 & 168.01 & 162.71 & 0.0190 & 1.045 & 1346.2 & 4.57 \\
\hline Kin 10 & 167.56 & 161.40 & 0.1089 & 1.095 & 1108.6 & 3.34 \\
\hline Kin 21 & 152.00 & 152.07 & 0.0175 & 1.094 & 853.1 & 6.15 \\
\hline
\end{tabular}

Table 7: Unseparated cross sections and corresponding kaon yields for $Q^{2}=1.90 \mathrm{GeV}^{2}$. Errors are statistical.

The cross sections determined using Eq. 43 together with the corresponding correction factors are listed in Tables 10 to 13 for each of the E98-108 kinematics. The center of mass cross sections listed in Tables 6 and 7, in Appendix A, were used to determine the longitudinal and transverse response functions by performing a Rosenbluth separation for each value of $Q^{2}$. 
The necessary condition for performing a Rosenbluth separation of $\sigma_{L}$ and $\sigma_{T}$ is that the interference terms of the cross sections cancel out and that the only contributing terms are the longitudinal, $\sigma_{L}$, and the transverse, $\sigma_{T}$ (see Eq. 9). The condition that the interference terms of the cross sections do not contribute to the total cross section is fulfilled for all kinematics as can be seen from the $\phi$ vs $t$ coverage shown in Appendix D. After determining the cross section, the longitudinal, $\sigma_{L}$, and the transverse, $\sigma_{T}$, response functions were extracted by performing a polynomial fit [PAW01] to the data (cross section versus the virtual photon polarization):

$$
p_{n}\left(x_{i}\right)=\sum_{i=0}^{n} a_{i} \cdot x_{i}^{n}
$$

where the degree, $n=1$ in our case. The coefficients $a_{i}$ are calculated such that the quantity

$$
S_{n}^{2}=\sum_{i=1}^{n}\left(y_{i}-p_{n}\left(x_{i}\right)\right)^{2}=\min
$$

An estimate $\sigma$ of the standard deviation of the fit is calculated with the standard formula [PAW01]:

$$
\sigma=\sqrt{\frac{S_{n}^{2}}{(n-1)}}
$$

The longitudinal and transverse cross sections are determined from the fitting results (see figures 128 to 135 ). The longitudinal part, $\sigma_{L}$, is the slope of the line and $\sigma_{T}$ is given by the intercept. The table with the results of the unseparated cross sections and all the applied corrections is listed in Appendix A. 


\begin{tabular}{|c|c|c|c|c|c|c|c|c|}
\hline Kin. & $E_{0}$ & $Q^{2}$ & W & $t_{\min }$ & $\sigma_{L}$ & $\delta_{\sigma_{L}}$ & $\sigma_{T}$ & $\delta_{\sigma_{T}}$ \\
\hline No. & $\mathrm{GeV}$ & $(\mathrm{GeV} / \mathrm{c})^{2}$ & $\mathrm{GeV}$ & $\mathrm{GeV}^{2}$ & $\mathrm{nb} / \mathrm{sr}$ & $\mathrm{nb} / \mathrm{sr}$ & $\mathrm{nb} / \mathrm{sr}$ & $\mathrm{nb} / \mathrm{sr}$ \\
\hline 1 & 5.6144 & 2.35 & 1.80 & -0.9498 & 53.405 & 10.822 & 104.261 & 17.194 \\
\hline 23 & 4.2383 & 2.35 & 1.80 & -0.9498 & 53.405 & 10.822 & 104.261 & 17.194 \\
\hline 20 & 3.4006 & 2.35 & 1.80 & -0.9498 & 53.405 & 10.822 & 104.261 & 17.194 \\
\hline 2 & 5.6144 & 2.35 & 1.85 & -0.8562 & 64.135 & 12.101 & 99.259 & 18.125 \\
\hline 24 & 4.2383 & 2.35 & 1.85 & -0.8562 & 64.135 & 12.101 & 99.259 & 18.125 \\
\hline 18 & 3.4006 & 2.35 & 1.85 & -0.8562 & 64.135 & 12.101 & 99.259 & 18.125 \\
\hline 3 & 5.6144 & 2.35 & 1.98 & -0.6737 & 50.931 & 12.480 & 109.904 & 21.442 \\
\hline 25 & 4.2383 & 2.35 & 1.98 & -0.6737 & 50.931 & 12.480 & 109.904 & 21.442 \\
\hline 4 & 5.6144 & 2.35 & 2.08 & -0.5716 & 68.714 & 6.811 & 89.344 & 13.066 \\
\hline 22 & 4.2383 & 2.35 & 2.08 & -0.5716 & 68.714 & 6.811 & 89.344 & 13.066 \\
\hline 11 & 5.7544 & 1.90 & 1.905 & -0.5994 & 64.959 & 11.570 & 125.490 & 16.952 \\
\hline 26 & 4.2383 & 1.90 & 1.905 & -0.6183 & 64.959 & 11.570 & 125.490 & 16.952 \\
\hline 19 & 3.4006 & 1.90 & 1.905 & -0.6183 & 64.959 & 11.570 & 125.490 & 16.952 \\
\hline 5 & 5.6144 & 1.90 & 1.94 & -0.5790 & 56.359 & 6.786 & 134.203 & 10.536 \\
\hline 27 & 4.2383 & 1.90 & 1.94 & -0.5790 & 56.359 & 6.786 & 134.203 & 10.536 \\
\hline 17 & 3.4006 & 1.90 & 1.94 & -0.5790 & 56.359 & 6.786 & 134.203 & 10.536 \\
\hline 12 & 5.7544 & 1.90 & 2.00 & -0.5203 & 80.475 & 15.911 & 108.038 & 23.037 \\
\hline 28 & 4.2383 & 1.90 & 2.00 & -0.5203 & 80.475 & 15.911 & 108.038 & 23.037 \\
\hline 10 & 5.6144 & 1.90 & 2.14 & -0.4143 & 36.864 & 14.303 & 134.645 & 21.293 \\
\hline 21 & 4.2383 & 1.90 & 2.14 & -0.4143 & 36.864 & 14.303 & 134.645 & 21.293 \\
\hline
\end{tabular}

Table 8: Separated cross sections. Errors are statistical and systematics added in quadrature (see appendix F). 


\subsubsection{Systematic uncertainties}

We determined the systematic uncertainties associated with the cross sections calculation using the studies from the sections tabulated in the third column of table 9 .

\begin{tabular}{|c|c|c|}
\hline Detector/Variable & Systematic error (\%) & Section \\
\hline Beam energy & 0.12 & (see Section 2.6) \\
A1 efficiency & 0.57 & (see Section 3.6) \\
$S_{1,2}^{E, H}$ efficiency & 1.33 & (see Section 3.2) \\
VDC efficiency & 1.97 & (see Section 3.5) \\
A2 efficiency & 0.87 & (see Section 3.7) \\
Charge & 0.3 & (see Section 2.3) \\
(see Section 3.3) \\
LH target density \\
Spectrometer acceptance \\
Background subtraction
\end{tabular}

Table 9: Systematic errors for the E981-08 experiment. 


\section{Conclusions}

The $Q^{2}$ dependence of the unseparated cross section was studied and compared with previous electroproduction data (see Fig. 75). There is good agreement between previous data and the E98-108 data. Figure 75 shows the qualitative agreement together with a phenomenological $Q^{2}$ fit. Data were taken at forward angles and exhibit a simple fall off with $Q^{2}$. The agreement is remarkable because the data were taken at various values of the virtual photon polarization $\epsilon$, the interference responses having various contributions, and at different $W$ values (data were extrapolated to a common $W$ value [BEB77]).

A comparison between separated response functions as extracted from the E98108 data (together with previous data) and models was studied. The $Q^{2}$ and $W$ dependence of the separated response functions is presented in figures 76 to 86 . The models we compare our data to, are described in Sec. 1.2. In the isobaric models, M2(WWW) and M2(WAA), the contributions from Born terms and extended Born terms corresponding to the exchange of a nucleon, hyperon and kaon (whether the reaction proceeds through the $s, t$, or $u$ channel respectively) and their resonances, are considered (see Fig. 3).

Each of the exchanged particles (nucleon, hyperon and kaon) contributes a propagator term to the transition matrix element (thus to the scattering amplitude). The propagator term is also the weighting factor for each diagram (channel). The difference between these two models (M2(WWW) and M2(WAA)) is in the way the 


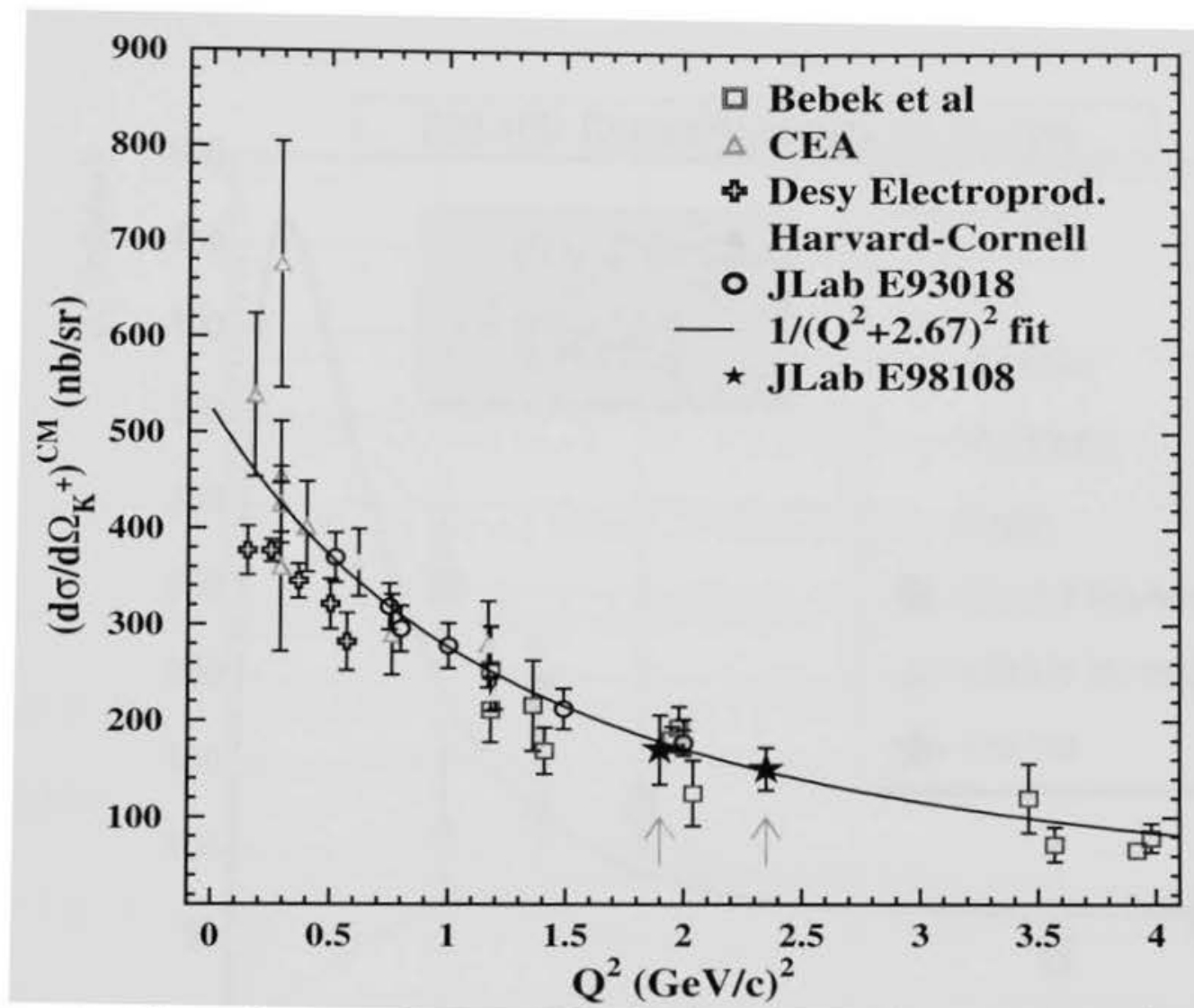

Figure 75: Kaon electroproduction: E98-108 data shown; $\Lambda$ cross sections (Kin 10 at $Q^{2}=1.90$ and at $W=2.14$, and Kin 1 at $Q^{2}=2.35$ and $W=1.80$ ) are extrapolated [BEB77] to $\mathrm{W}=2.15 \mathrm{GeV}$.

electromagnetic form factors are modeled. The M2(WWW) model uses a vector meson dominance model (the virtual photon is considered to be coupling directly to a virtual vector meson that interacts with the hadron) for the $t$ channel exchanges, and the coupling constants are obtained by fits to previous electroproduction data. The M2(WAA) model uses an extended vector meson dominance approach (for the 


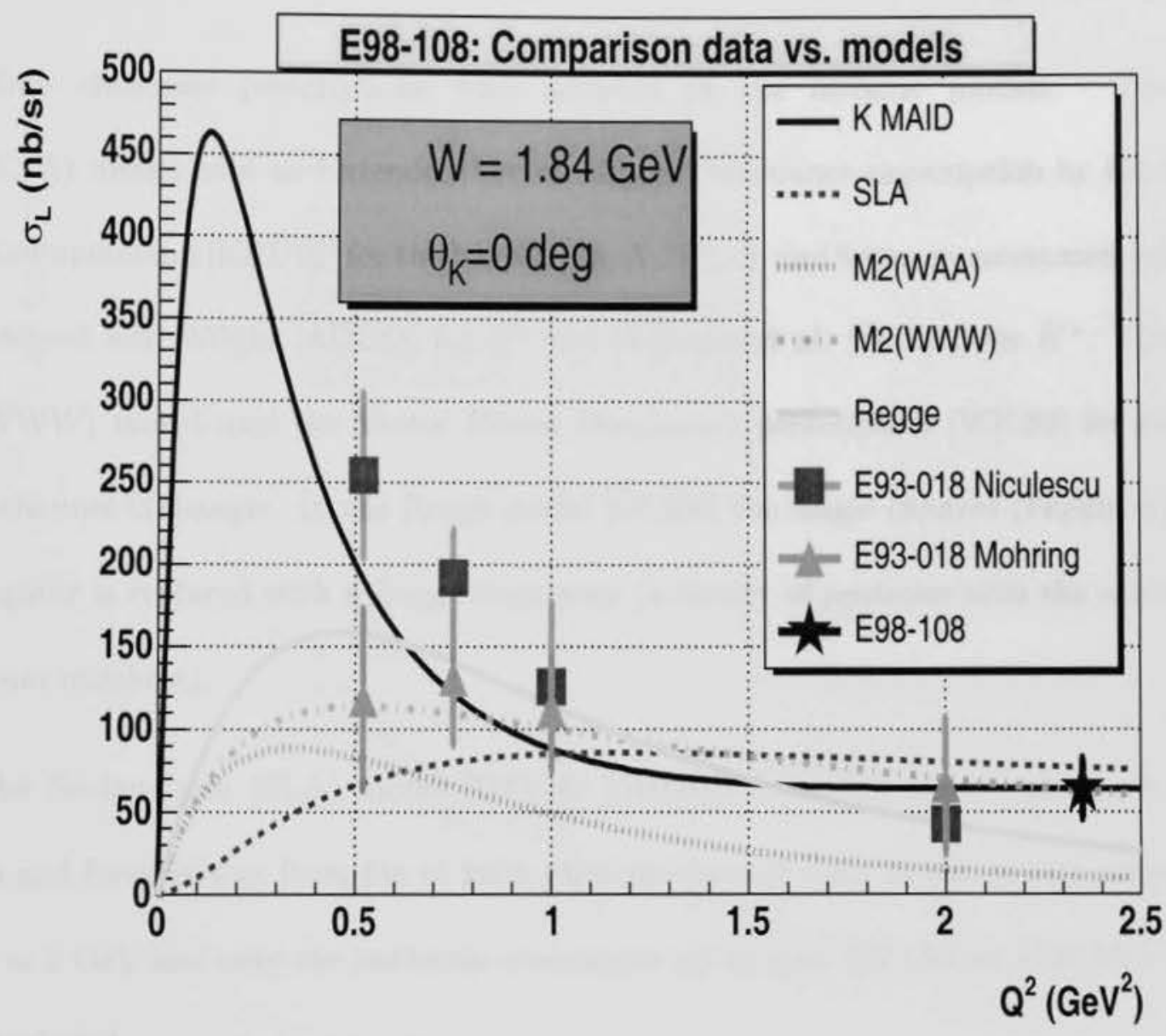

Figure 76: Longitudinal cross section, $\sigma_{L}^{\Lambda}$ versus $Q^{2}$ for $W=1.84$, comparison E98108 data and available models. Theoretical calculations from [BYD04], solid line is K-Maid [MAR03], dotted bold line is SLA [DAV96], [MIZ98] double dotted line is M2(WAA) [WIL92], dotted-interrupted line is M2(WWW) [ADE88], dotted line is Regge [GUI00], the square symbol is data from [NIC98], the triangle is data from [MOH03] and the star is E98-108 data. 
$t$ channel exchanges) to model the kaon electromagnetic form factor and considers that the virtual photon interacts directly with the hadron's constituent quarks.

More elaborate prescriptions were adopted in the isobaric models. The M2(WAA) model uses an Extended Vector Meson Dominance prescription by Gari and Krumpelmann [KR $\ddot{U} 92]$ for the baryons $\left(\mathrm{p}, \Lambda, \mathrm{N}^{*}, \ldots\right)$ and forms as recommended by Adelseck and Wright [ADE88] for $K^{*}$ and Williams et al. [WIL92] for $K^{+}$. The M2(WWW) model uses the Vector Meson Dominance prescription [WIL92] for all the t-channel exchanges. In the Regge model [GUI00] the single channel (Feynman) propagator is replaced with a Regge trajectory (a family of particles with the same quantum numbers).

The Saclay-Lyon (SLA) model [DAV96] [MIZ98], extracted the coupling constants and form factors from fits to kaon photoproduction data in the energy range up to $\approx 2 \mathrm{GeV}$ and only the nucleonic resonances up to spin $3 / 2(\mathrm{~N} 7$ at $1720 \mathrm{MeV})$ were included.

In Figs. 76 and 77 the separated longitudinal, $\sigma_{L}^{\Lambda}$, and transverse, $\sigma_{T}^{\Lambda}$, response functions are plotted as a function of $Q^{2}$ (at a $W$ value of $1.84 \mathrm{GeV}$ ) together with the models mentioned above. There is an overlap, at a $Q^{2}$ of $1.9 \mathrm{GeV}^{2}$ (but at different $W$ values), with the data taken at JLab by the E93-018 experiment [NIC98] [MOH03]. Both the longitudinal, $\sigma_{L}^{\Lambda}$, and transverse, $\sigma_{T}^{\Lambda}$, terms fall off with increasing $Q^{2}$. The E98-108 data lies in a region where the difference between the K-Maid, the Regge and the SLA models is not very significant and as such do not allow for 


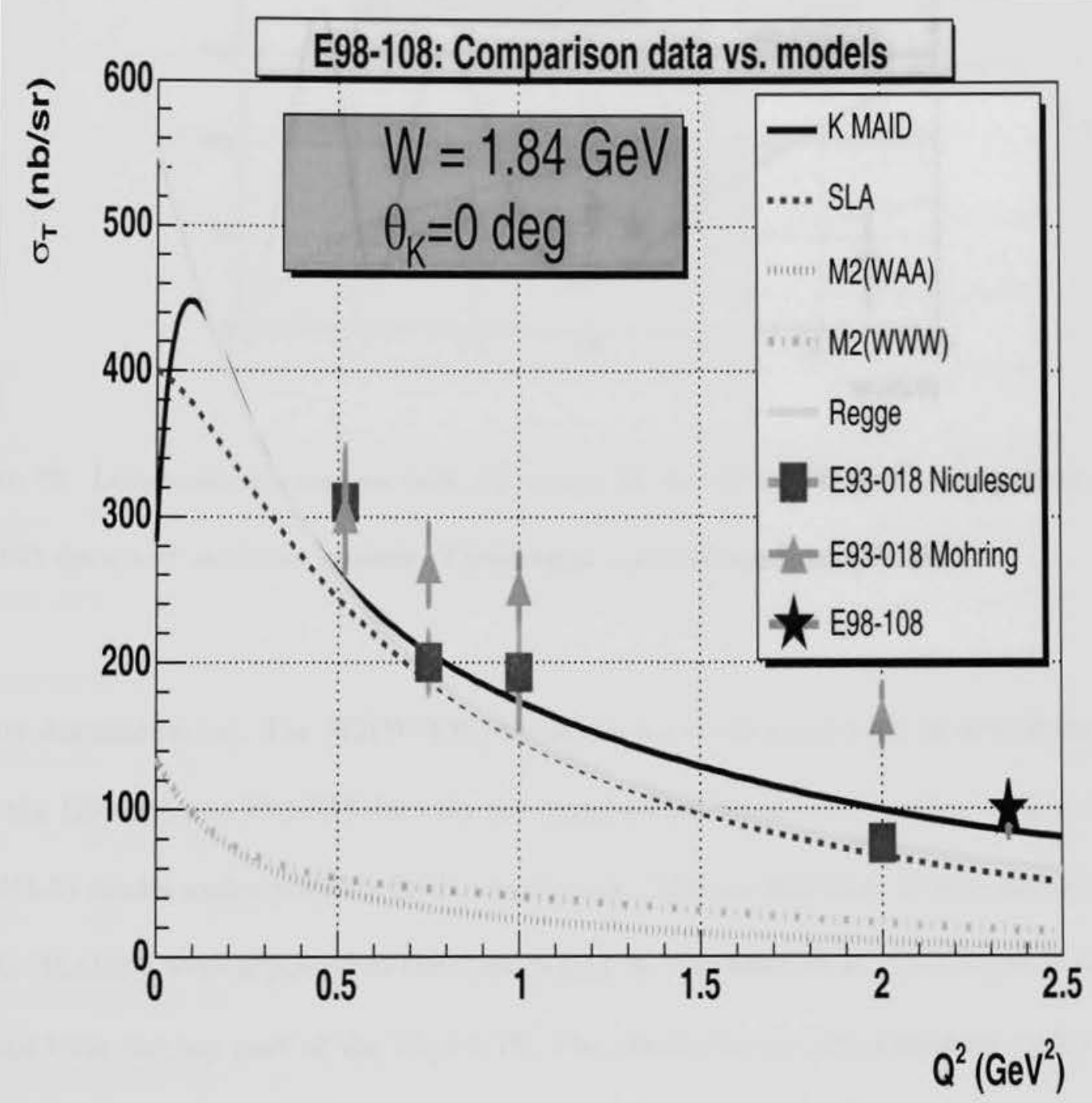

Figure 77: Transverse cross section, $\sigma_{T}^{\Lambda}$ versus $Q^{2}$ for $W=1.84$, comparison E98-108 data and available models. Theoretical calculations from [BYD04]. 


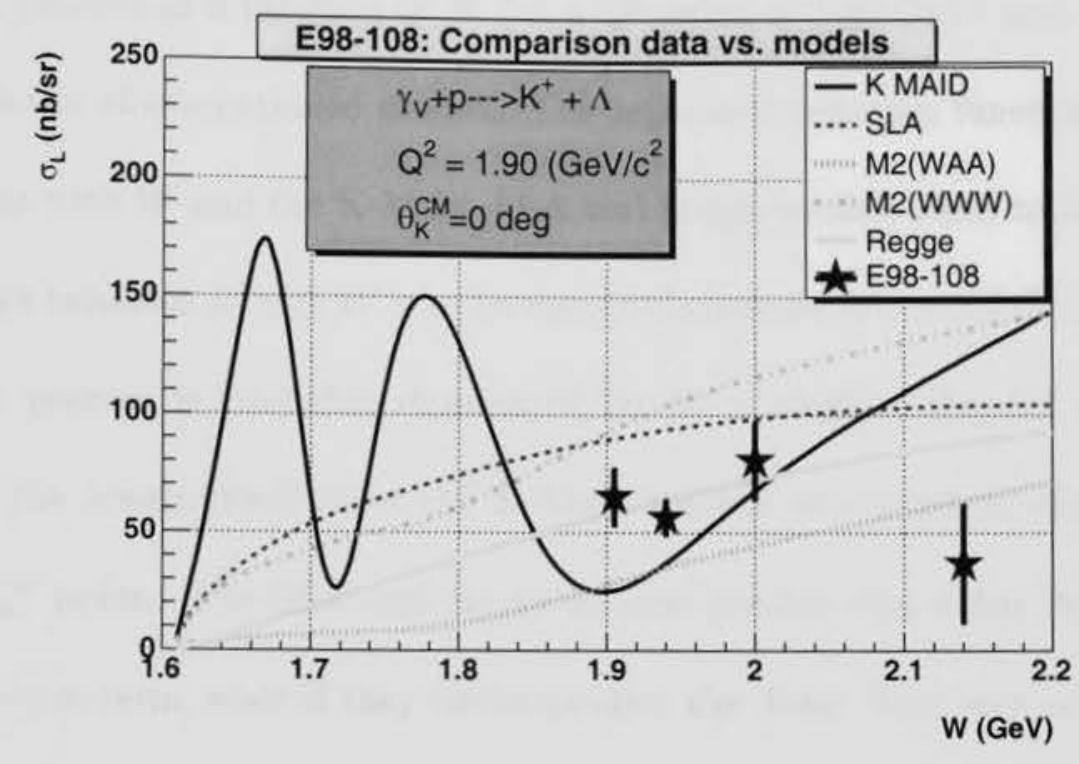

Figure 78: Longitudinal cross section, $\sigma_{L}^{\Lambda}$ versus $W$ for $Q^{2}=1.90 \mathrm{Gev}^{2}$, comparison E98-108 data and available models. Theoretical calculations from [BYD04].

further discrimination. The M2(WWW) model seems to do a good job in describing both the E98-108 and E93-018 data for the longitudinal term, but together with the M2(WAA) model under-predicts both sets of data. The models SLA, Regge, and KMAID [MAR03] were adjusted to the data points by Niculescu et al., [NIC98] as it is obvious from the top part of the Figure 76 . The results for $\sigma_{T}$ are consistent rather with the data by Niculescu et al., but are systematically below the later analysis done by Mohring, and below the E98-108 data. In the case of the Regge model, the electromagnetic (EM) form factors at the photon vertexes were determined by fitting the data of Niculescu et al. [NIC98] (see Fig. 76, Fig. 77).

In Figs. 78 and 79 the separated longitudinal, $\sigma_{L}^{\Lambda}$, and transverse, $\sigma_{T}^{\Lambda}$, response 
functions are plotted as a function of $W$ (at a $Q^{2}$ value of $1.90 \mathrm{GeV}^{2}$ and $\theta_{C M}=0$ ) together with the aforementioned models. The separated response functions exhibit a flat behavior with $W$ and the K-Maid, SLA and Regge models seem to follow that trend. The flat behavior at high $W$ is to be expected since we are out of the resonance region. The process is probably dominated by by a single $t$-channel exchange. However for the longitudinal term the K-Maid models over-predicts the data at the highest $Q^{2}$ point. The SLA and Regge models predict also a flat dependence for the transverse term, even if they under-predict the data. The very pronounced resonance structure at low $W$ values displayed by the K-Maid model is due to the inclusion of the resonance at $1720 \mathrm{GeV}$. The K-Maid model was not designed to describe experimental data in the low $W$ regions and was not properly tested for electroproduction processes at forward angles. In Figs. 80 and 81 the separated longitudinal, $\sigma_{L}^{\Lambda}$, and transverse, $\sigma_{T}^{\Lambda}$, response functions are plotted as a function of $W$ for our highest $Q^{2}$ value, $2.35 \mathrm{GeV}^{2}$, and $\theta_{C M}=0$, and compared with the same models. The Regge model is the model that best describes both the longitudinal and the transverse data (the transverse term is under-predicted as can be seen from Fig. 81). K-Maid does seem to follow the data in the high $Q^{2}$ region (above $\approx 1.84$ $\mathrm{GeV})$.

An estimate of the kaon form factor has been explored (see Fig. 82 to 85 ). The estimate was obtained by determining the sensitivity of the separated cross sections to variations of the kaon EM form factor. The cross sections have been calculated using the Regge model [BYD04]. At an angle of $0^{\circ}$, the longitudinal cross section is 


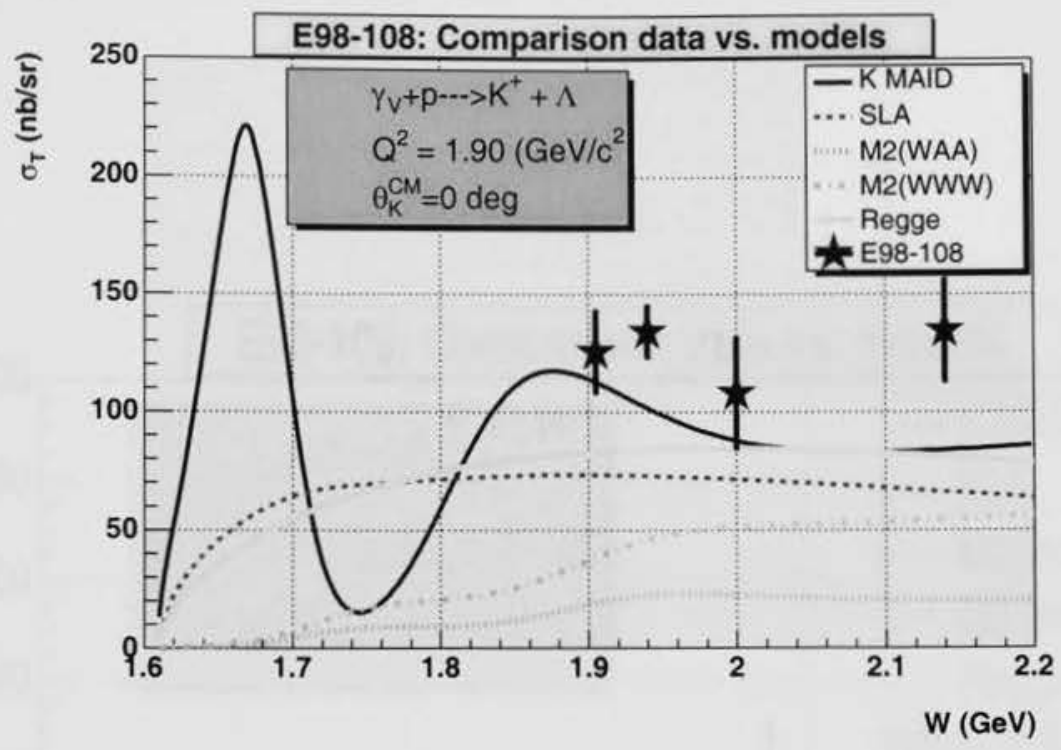

Figure 79: Transverse cross section, $\sigma_{T}^{\Lambda}$ versus $W$ for $Q^{2}=1.90$, comparison E98-108 data and available models. Theoretical calculations from [BYD04].

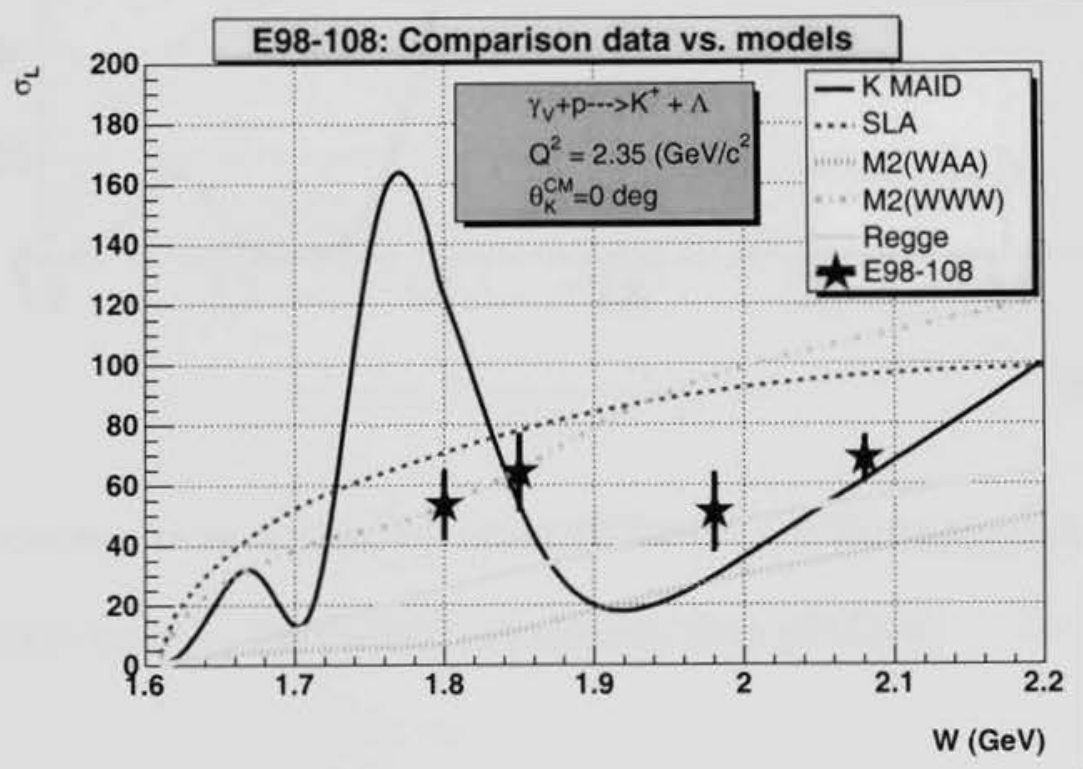

Figure 80: Longitudinal cross section, $\sigma_{L}^{\Lambda}$ versus $W$ for $Q^{2}=2.35$, comparison data and available models. Theoretical calculations from [BYD04]. 


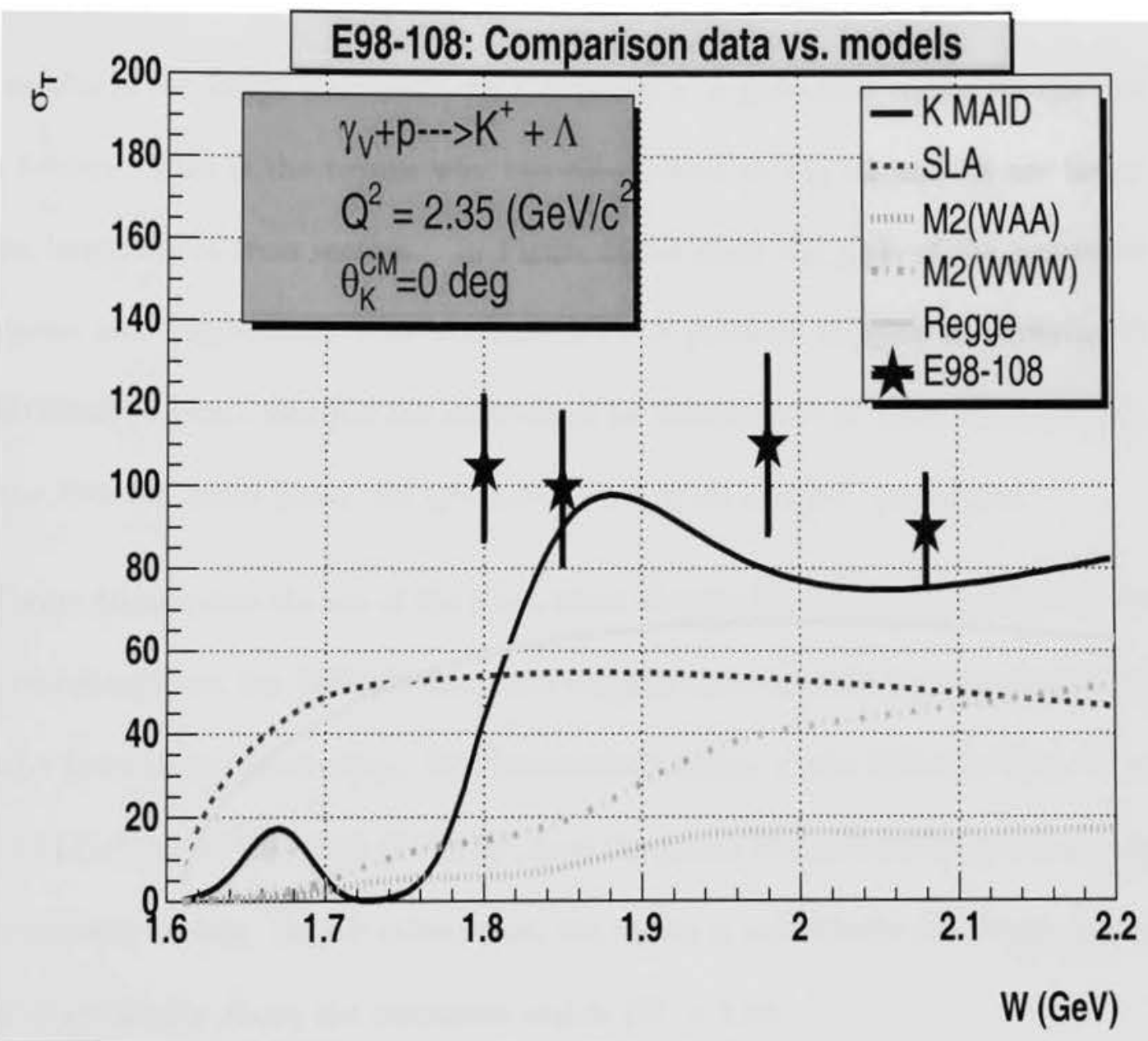

Figure 81: Transverse cross section, $\sigma_{T}^{\Lambda}$ versus $W$ for $Q^{2}=2.35$, comparison E98-108 data and available models. Theoretical calculations from [BYD04]. 
given by a superposition of the amplitudes (CGNL amplitudes [DAV96]) multiplied by a kinematical factor $C$ :

$$
\sigma_{L}\left(\theta_{K}=0\right)=C|f 1+f 3+f 4+f 5+f 6|^{2} .
$$

The results of the Regge model are quite sensitive to a particular choice for the EM form factors. This is the reason why the effects seen in Fig 82 and 84 are larger for the longitudinal cross section. In Figure 86 we show the ratio of the separated transverse and longitudinal cross sections, $L / T$. A previous analysis by Mohring et al. [MOH03] (square), and (for the same data) by Niculescu et al. [NIC98] (triangle), and the E98-108 point (star), for $Q^{2}=2.35 \mathrm{GeV}^{2}, W=1.84 \mathrm{GeV}$, are shown.

Figure 86 supports the use of the Kaon Maid and the Regge models as the models that reproduce best the E98-108 data and suggest these models are appropriate to describe kaon electroproduction. The kinematical region of the E98-108 data, $\mathrm{W}=$ $1.8-2.14 \mathrm{GeV}, Q^{2}=1.9-2.35(\mathrm{GeV} / \mathrm{c})^{2}$, is at the limits of a reasonable applicability of the isobaric models. On the other hand, the region is suitable for the Regge model which is applicable above the resonance region $(W>1.9)$.

Both the SLA and Regge models give reasonable results in predicting the $\sigma_{T}$ response function even if they under-predict the longitudinal part. Both models exhibit a flat $W$-dependence of the $\sigma_{T}$ and $\sigma_{L}$ as the data require [BYD04]. The longitudinal cross section seem to be better described by these two models (SLA and Regge). As for the M2(WWW) and M2(WAA) models, they both predict a smaller cross sections (for both the longitudinal and transverse terms as seen in Fig. 76 and 


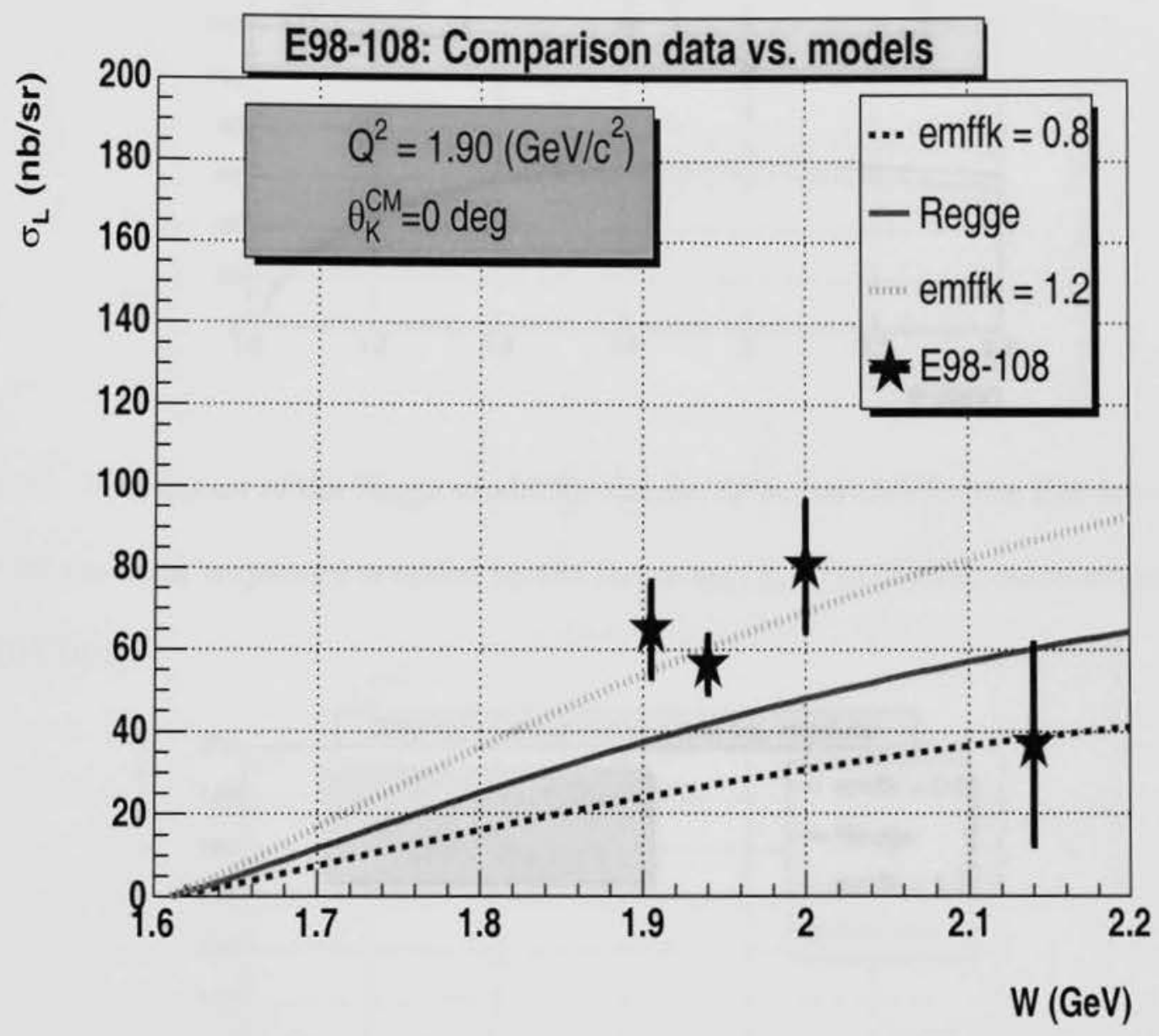

Figure 82: Predictions of the Regge model for the $\sigma_{L}, Q^{2}=1.90 \mathrm{GeV}^{2}$. The EM form factor for the kaon trajectory is scaled by the factor $\operatorname{emf} f_{k}$. Theoretical calculations from [BYD04], solid line is Regge [GUI00], the square symbol is data from [MOH03], the triangle is data from [NIC98] and star is E98-108 data. 


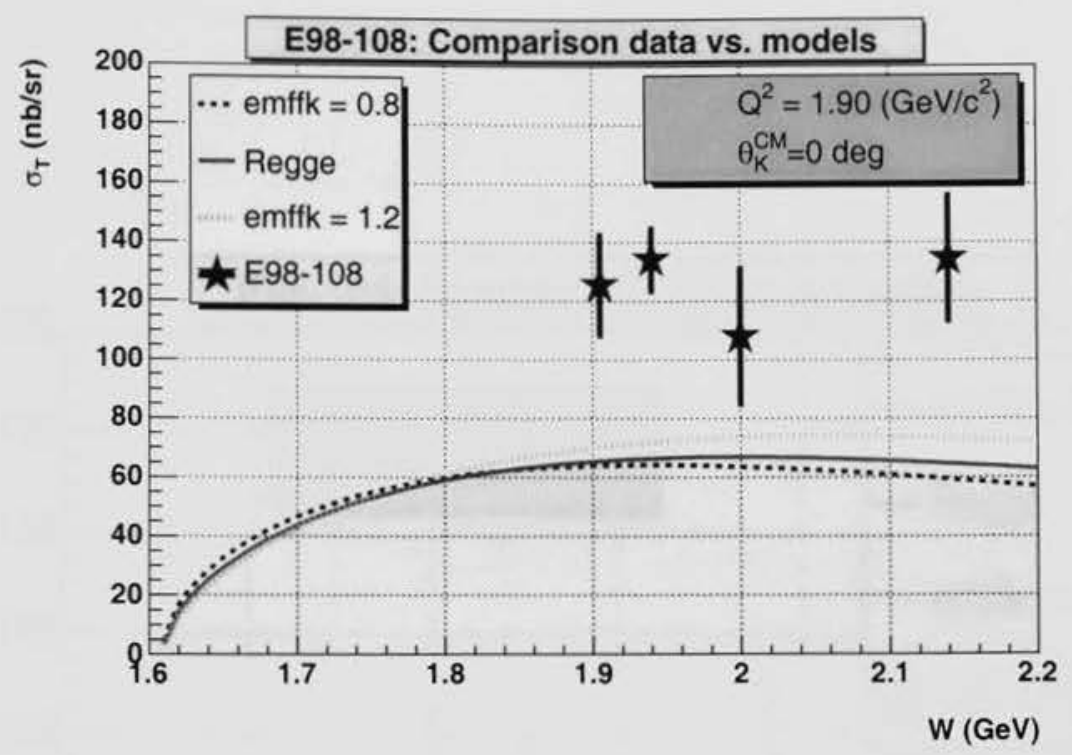

Figure 83: Predictions of the Regge model for the $\sigma_{T}, Q^{2}=1.90 \mathrm{GeV}^{2}$. The EM form factor for the kaon trajectory is scaled by the factor $\operatorname{emf} f_{k}$. Theoretical calculations from [BYD04].

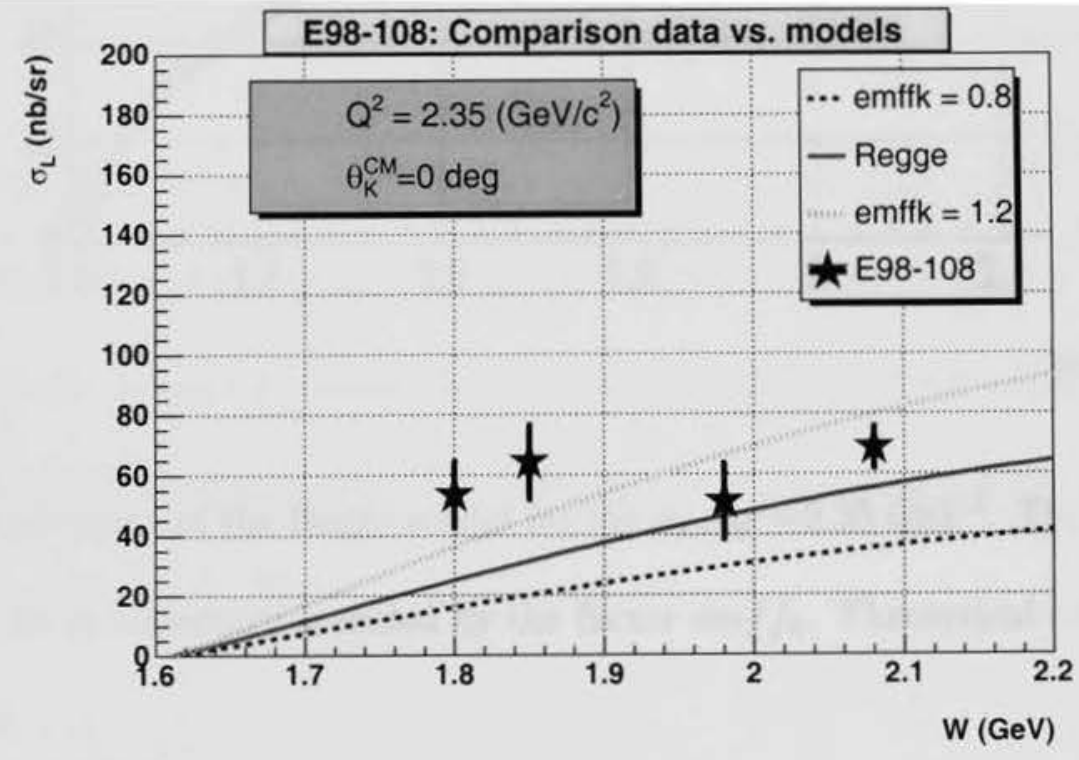

Figure 84: Predictions of the Regge model for the $\sigma_{L}, Q^{2}=2.35 \mathrm{GeV}^{2}$. The EM form factor for the kaon trajectory is scaled by the factor $\operatorname{emf} f_{k}$. Theoretical calculations from [BYD04]. 


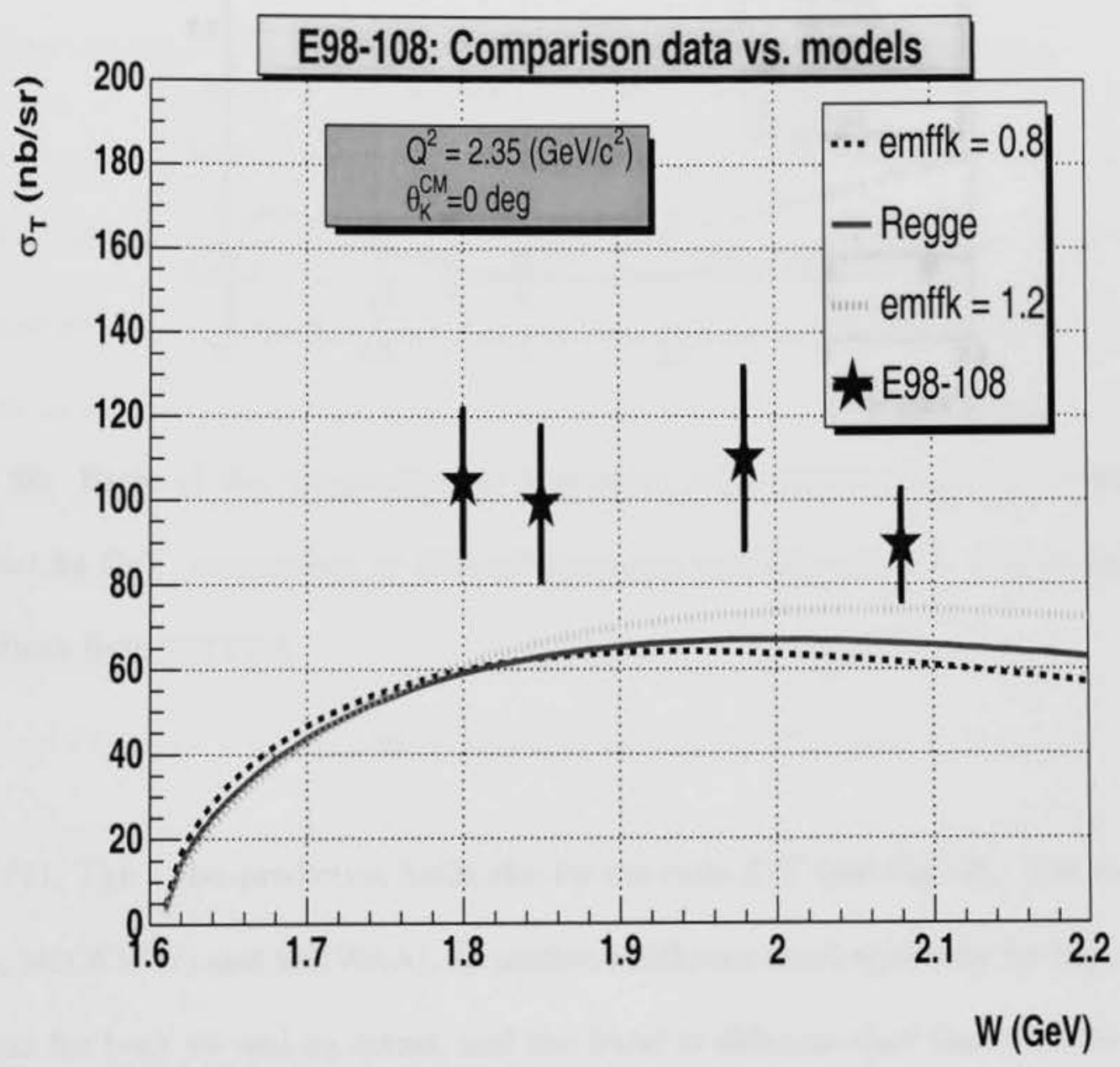

Figure 85: Predictions of the Regge model for the $\sigma_{T}, Q^{2}=2.35 \mathrm{GeV}^{2}$. The EM form factor for the kaon trajectory is scaled by the factor $\operatorname{emff} f_{k}$. Theoretical calculations from [BYD04]. 


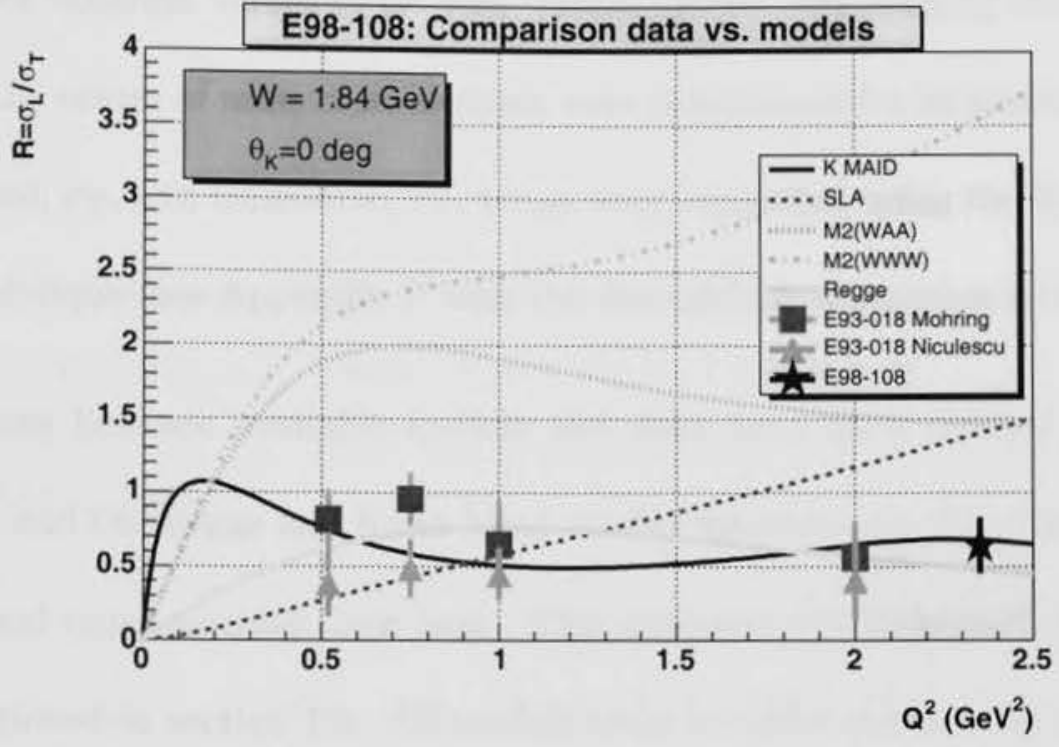

Figure 86: Ratio of the longitudinal to transverse cross section $\left(\sigma_{L} / \sigma_{T}\right)^{\Lambda}$ versus $Q^{2}, W=1.84 \mathrm{GeV}$, comparison of E98-108 data and available models. Theoretical calculations from [BYD04].

in Fig. 77). The under-prediction holds also for the ratio $L / T$ (see Fig. 86). The two models, M2(WWW) and M2(WAA), do predict a different trend especially for higher $W$ values for both $\sigma_{T}$ and $\sigma_{L}$ terms, and the trend is different than that described by the SLA and the Regge models. The difference between the models seems to be more significant for the $\sigma_{L}$ part.

\subsection{Summary}

The kaon electroproduction reaction $\mathrm{H}\left(e, e^{\prime} K+\right) Y$ was studied as a function of the four momentum transfer, $Q^{2}$, for different values of the virtual photon polarization 
parameter. For different values of $Q^{2}$ (see Tables 10 to 13) ranging from 1.90 to $2.35 \mathrm{GeV} / \mathrm{c}^{2}$ the center of mass cross sections were determined for 20 kinematics and the longitudinal, $\sigma_{T}$, and transverse, $\sigma_{T}$, terms were separated using the Rosenbluth separation technique (see Appendix F with the Rosenbluth separation results).

Comparisons between available models and data have been studied (see Figures 78 to 86 ) and the Regge and Kaon Maid models qualitatively describe the E98108 longitudinal cross sections data best. This supports the t-channel dominance behavior mentioned in section 1.2. All models seem to under-predict the transverse cross section. The results of this study will provide a more solid base for the transition from a semi-phenomenological description of kaon electroproduction in terms of mesons and baryons to a pQCD-based description in terms of quarks (or diquarks). The data from the E98-108 experiment extend the range of the available kaon electroproduction cross section data into a region of $Q^{2}$ where no separations have ever been performed (see Fig. 75). 


\section{References}

[ABR71] R.J.Abrams et. al., Phys. Rev. D 4, (1971).

[ADE88] R. A. Adelseck, L.E. Wright, Phys. Rev. C 38, (1988).

[ALC04] John Alcorn et. al., Basic Instrumentation for Hall A at Jefferson Jab, NIM A 522, (2004).

[ARM03] D.S. Armstrong, B. Moffit, and R. Suleiman, Target Density Fluctuations and Bulk Boiling in the Hall A Cryotarget, JLAB-TN-03-017, (2003).

[BEB77] C.J. Bebek et. al., Phys. Rev. D 15, 594 (1977).

[BOE02] Werner U. Boeglin, Florida International University, private communication, (2002).

[BYC03] P. Bydžovský, F. Cusanno, S. Frullani, F. Garibaldi, M. Iodice, M. Sotona, G.M. Urcioli, Los Alamos Preprint server, http://xxx.lanl.gov/ps/nuclth/0305039, (2003).

[BYS03] Petr Bydžovský, Miloslav Sotona, Los Alamos Preprint server, http://xxx.lanl.gov/ps/nucl-th/0408039, (2003).

[BYD04] Petr Bydžovský, Miloslav Sotona, Nuclear Physics Institute, Řě near Prague, Czech Republic, private communication, (2004).

[CLO79] F. E. Close, An introduction to quarks and particles, Academic Press, New York, (1979).

[DAV96] J.C. David, C. Fayard, G.H. Lamot, B. Saghai, Phys. Rev. C, 532613 (1996).

[EID04] S. Eidelman et. al., http://pdg.lbl.gov/2004/html/authors_2004.html Phys. Lett. B 592, 1 (2004).

[ESP02] ESPACE: Event Scanning program for Hall-A Collaboration experiments, The HallA collaboration, http://hallaweb.jlab.org/espace, (2002).

[FEY72] R.P. Feynman, Photon Hadron Interactions, Benjamin Inc., (1972)

[FIS92] K. G. Fissum et. al., JLAB-TN-00-016, (2000).

[KRÜ92] M. F. Gari and W. Krümpelmann, Phys. Rev D 45, 1817, (1992).

[GAV02] A. Gavalya, private communication, (2002). 
[GNU03] Gnumeric, http://www.gnome.org/projects/gnumeric, (2003).

[GUI00] M. Guidal, J.-M. Laget, and M. Vanderhaeghen, Electroproduction of strangeness above the resonance region, Phys. Rev. C 61, 025204, (2000).

[HAU86] R. Hausmann and W. Weise, Z. Phys. A 324, 355 (1986).

[HWEBA] http://www.jlab.org/help/Ghelp/halla3d.html

[JAN01] S. Janssen, J. Ryckebusch, D. Debruyne, and T. V. Cauteren, Phys. Rev. C65, (2001).

[JEF03] Jefferson Lab alignment group, http://hallaweb.jlab.org/news/minutes/Survey_Reports, (2003).

[LER02] John LeRose, R-functions for the HRS, http://www.jlab.org/ ^lerose, (2002).

[LIY02] Nilanga Liyanage, Optics Calibration of the Hall A High Resolution Spectrometers, JLAB-TN-02-012, (2002).

[LYO99] Louis Lyons, Statistics for Nuclear and Particle Physicists, Cambridge Univeristy Press, (1999).

[PRO98] P. Markowitz, M. Iodice, S. Frullani, C.C. Chang, O.K. Baker, Jefferson Lab E98-108 proposal, (1998).

[MAR02] Pete E. C. Markowitz, Code to calculate $p\left(e, e^{\prime} K^{+}\right) \Lambda$ cross-sections based on P. Brauel et.al., Z.Phys. C 3, 101 (1979), private communication, (2002).

[MAR03] T. Mart and C. Bennhold, Kaon elementary cross sections, http://www.kph.uni-mainz.de/MAID/kaon/kaonmaid.html, (2003).

[MIC02] Robert Michaels, Trigger Programming for Hall A Spectrometers, http://hallaweb.jlab.org/equipment/daq/daq_trig.html, (2002).

[MIC01] Robert Michaels, Bodo Reitz, James Proffitt, Update on electronics deadtime, JLAB, http://www.jlab.org/ rom/edt_annrpt_2001.ps, (2001).

[MCN04] J.W.C. McNabb et. al., Phys. Rev. C69, 042201(R) (2004).

[MIZ98] T. Mizutani, C. Fayard, G.-H. Lamot, and B. Saghai, Phys. Rev. C 58, 75, (1998).

[MOH03] R.M.Mohring, et. al., Separation of the longitudinal and transverse cross sections in the ${ }^{1} H\left(e, e K^{+}\right) \Lambda$ and ${ }^{1} H(e, e K+) \Sigma^{0}$ reactions, Phys. Rev. C 67, 055205 (2003). 
[NAS04] R. Nasseripour, Measurement of the single spin asymmetry and the polarized structure function in the exclusive $p\left(e, e^{\prime} K^{+}\right) \Lambda$ reaction with CLAS, $\mathrm{PhD}$. Thesis, Florida International University, (2004).

[NIC98] Gabriel Niculescu et. al., Phys. Rev. Lett. 81, 1805 (1998).

[PAW01] PAW: The Physics Analysis Workstation, http://wwwasd.web.cern.ch/wwwasd/paw, (2001).

[PIC89] A. Picklesimer and J. Van Orden, Phys. Rev. C, 40, 290 (1989).

[RAU05] Brian A. Raue, Florida International University, private communication, (2005).

[REJ03] J.Reinhold, private communication, (2003).

[REB03] Bodo Reitz, Jefferson Lab, private communication (2003).

[ROO03] ROOT: An Object-Oriented Analysis Framework, http://root.cern.ch, (2003).

[RVA00] Marat Rvachev, Effective use of JLab Hall A HRS acceptance with Rfunctions, JLAB-TN-01-055, (2000).

[ULM02] P.E. Ulmer, MCEEP: Monte Carlo for electro-nuclear coincidence experiments, version 3.7, http://hallaweb.jlab.org/data_reduc/mc, (2002).

[WIL93] Robert A. Williams Jr., A crossing and duality consistent model of $\Lambda, \Sigma_{0}$ and $\Lambda(1405)$ Hyperon formation in kaon electromagnetic production and capture reactions, $\mathrm{PhD}$. Thesis, North Carolina State University, (1993).

[WIL92] R. A. Williams, C-R Ji and S. R. Cotanch, Phys. Rev. C 46, 1617 (1992), F.X. Lee, T. Mart, C. Bennhold, H. Haberzettl, L.E. Wright, nucl-th/9907119, T. Mart, C. Bennhold, Phys. Rev. C61 (2000) 012201, C. Bennhold et. al., nuclth/0008024, C. Bennhold et. al., nucl-th/9909022.

[WOJ01] Bogdan Wojtsekhowski, Threshold Cherenkov counters for Pions and Kaons, poster presented at the LBL International Nuclear Physics Conference, Berkeley, California, (2001).

[WOJ02] Bogdan Wojtsekhowski, Thomas Jefferson National Lab., private communication, (2002). 


\section{Appendices}

\section{A Unseparated cross sections and correction factors for E98-108 experiment}

\begin{tabular}{|c|c|c|c|c|c|}
\hline Kin. no. & KIN 1 & KIN 23 & KIN 20 & KIN 10 & KIN 21 \\
\hline \multicolumn{6}{|l|}{ Mceep rad. } \\
\hline$d^{6} \sigma\left(\frac{f m^{2}}{M e V^{2} s r^{2}}\right)$ & $1.664 \mathrm{E}-13$ & $5.851 \mathrm{E}-14$ & $2.904 \mathrm{E}-14$ & $3.084 \mathrm{E}-13$ & $1.104 \mathrm{E}-13$ \\
\hline Yield $(\mathrm{Hz})$ & 0.0306 & 0.0067 & 0.0015 & 0.1130 & 0.0175 \\
\hline \multicolumn{6}{|l|}{ Mceep no rad. } \\
\hline$d^{5} \sigma\left(\frac{f m^{2}}{M e V s r^{2}}\right)$ & $2.361 \mathrm{E}-11$ & $+8.533 \mathrm{E}-12$ & $3.069 \mathrm{E}-12$ & $3.744 \mathrm{E}-11$ & $1.177 \mathrm{E}-11$ \\
\hline Yield $(\mathrm{Hz})$ & 0.0319 & 0.0071 & 0.0017 & 0.1237 & 0.0192 \\
\hline Rad. Correction & 1.0411 & 1.0539 & 1.0843 & 1.0946 & 1.0939 \\
\hline \multicolumn{6}{|l|}{ KFIT2 } \\
\hline$d^{5} \sigma \frac{f m^{2}}{M e V s r^{2}}$ & $2.494 \mathrm{E}-11$ & $+8.971 \mathrm{E}-05$ & $3.189 \mathrm{E}-05$ & $4.093 \mathrm{E}-11$ & $1.220 \mathrm{E}-11$ \\
\hline$\frac{d \sigma}{d \Omega}(\mathrm{nb} / \mathrm{sr}) \mathrm{kfit} 2$ & 148.8158 & 138.8833 & 125.6707 & 167.5606 & 152.0038 \\
\hline Survival fraction kfit & 0.1478 & 0.1478 & 0.1478 & 0.2671 & 0.2671 \\
\hline Photon Flux * Jacob & $1.134 \mathrm{E}-12$ & $4.370 \mathrm{E}-06$ & $1.717 \bar{E}-06$ & $+9.145 \mathrm{E}-13$ & $3.005 \mathrm{E}-13$ \\
\hline Yield NO RFN cut & 1352.80 & 416.10 & 604.70 & & \\
\hline Yield RFN +0.008 & 339.10 & 481.10 & 159.80 & 1108.60 & 853.10 \\
\hline Eq. time at $75 \mu A$ & $2.15 \mathrm{E}+04$ & $1.48 \mathrm{E}+05$ & $2.16 \mathrm{E}+05$ & $1.86 \mathrm{E}+04$ & $+7.95 \mathrm{E}+04$ \\
\hline Rad. data $Y_{N O R F N}$ & 0.0628 & 0.0028 & 0.0028 & 0.01 & 0.00 \\
\hline Rad. data $Y_{P O Z R F N}$ & 0.0157 & 0.0033 & 0.07 & 0.0597 & 0.0107 \\
\hline \multicolumn{6}{|l|}{ Corrections due to } \\
\hline TOF window cut & 1.0091 & 1.0116 & 1.0055 & 1.0470 & 1.0026 \\
\hline EDT Correction & 1.0399 & 1.0227 & 1.0462 & 1.0329 & 1.0331 \\
\hline VDC Corr. Factor & 1.6388 & 1.6068 & 1.6883 & 1.4045 & 1.3778 \\
\hline A1 corr. factor & 1.019 & 1.019 & 1.019 & 1.019 & 1.019 \\
\hline A2 corr. factor & 1.0224 & 1.0224 & 1.0224 & 1.0017 & 1.0017 \\
\hline Luminosity $\left(n b^{-1}\right)$ & $6.66 \overline{\mathrm{E}}+09$ & $4.57 \mathrm{E} 10$ & $6.66 \mathrm{E} 10$ & $5.74 \mathrm{E}+09$ & $2.46 \mathrm{E} 10$ \\
\hline Kaon Absorbtion & 1.0466 & 1.0466 & 1.0466 & 1.0444 & 1.0452 \\
\hline Gas Cerenkov & 1.0227 & 1.0227 & 1.0227 & 1.0227 & 1.0227 \\
\hline Target Boiling & 1.0250 & 1.0800 & 1.0800 & 1.10 & 1.0500 \\
\hline Corrected Yield $(\mathrm{Hz})$ & 0.0309 & 0.0065 & 0.0016 & 0.1089 & 0.0175 \\
\hline$\frac{d \sigma}{d \Omega}(\mathrm{nb} / \mathrm{sr})$ & 150.5322 & 134.7110 & 130.2736 & 161.3978 & 152.0698 \\
\hline Data/Mceep & 1.0115 & 0.9700 & 1.0366 & 0.9632 & 1.04 \\
\hline
\end{tabular}

Table 10: Unseparated cross sections and corresponding correction factors: Kin 1, 23, 20, 10 and 21; The factor Rad. Correction represents the ratio of the yields as obtained from running the Monte Carlo code [ULM02] with the radiative corrections turned off and with the corrections turned on. 


\begin{tabular}{|c|c|c|c|c|c|}
\hline Kin. no. & KIN 2 & $\overline{\text { KIN } 24}$ & KIN 18 & KIN 3 & KIN 25 \\
\hline \multicolumn{6}{|l|}{ Mceep rad. } \\
\hline$d^{6} \sigma\left(\frac{f m^{2}}{M e V^{2} s r^{2}}\right)$ & $1.8061 \mathrm{e}-13$ & $6.03 \mathrm{e}-14$ & $2.9877 \mathrm{e}-14$ & $1.950 \mathrm{E}-13$ & $5.997 \mathrm{E}-14$ \\
\hline Yield $(\mathrm{Hz})$ & 0.0377 & 0.0081 & 0.0016 & 0.0567 & 0.0094 \\
\hline \multicolumn{6}{|l|}{ Mceep no rad. } \\
\hline$d^{5} \sigma\left(\frac{\overline{f m^{2}}}{M e V s r^{2}}\right)$ & $2.5301 \mathrm{e}-11$ & $8.8054 \mathrm{e}-12$ & $2.9577 \mathrm{e}-12$ & $2.668 \mathrm{E}-11$ & $+8.380 \mathrm{E}-12$ \\
\hline Yield $(\mathrm{Hz})$ & 0.0410 & 0.0084 & 0.0017 & 0.0624 & 0.0099 \\
\hline Rad. Correction & 1.0869 & 1.0440 & 1.0980 & 1.10 & 1.0556 \\
\hline \multicolumn{6}{|l|}{ KFIT2 } \\
\hline$d^{5} \sigma \frac{f m^{2}}{M e V s r^{2}}$ & $2.648 \mathrm{e}-11$ & $9.2205 \mathrm{e}-12$ & $3.077 \mathrm{e}-12$ & $2.770 \mathrm{E}-11$ & $+8.706 \mathrm{E}-12$ \\
\hline$\frac{d \sigma}{d \Omega}(\mathrm{nb} / \mathrm{sr}) \mathrm{kfit} 2$ & 151.8385 & 140.8938 & 126.4472 & 148.7611 & 135.6547 \\
\hline Survival fraction kfit & 0.1724 & 0.1724 & 0.1724 & 0.2302 & 0.2302 \\
\hline Photon Flux * Jacob & $1.0115 \mathrm{e}-12$ & $3.7958 \mathrm{e}-13$ & $1.4114 \mathrm{e}-13$ & $+8.088 \mathrm{E}-13$ & $2.788 \mathrm{E}-13$ \\
\hline Yield NO RFN cut & 2124.40 & & & 3609.30 & \\
\hline Yield RFN +0.008 & 531.2 & 546.6 & 175.70 & 914.70 & 676.10 \\
\hline Eq. time at $75 \mu \mathrm{A}$ & 27124.92 & 135244.355 & 226724.305 & $3.11 \mathrm{E}+04$ & $1.31 \mathrm{E}+05$ \\
\hline Rad. data $Y_{N O R F N}$ & 0.0783 & 0.00 & 0.00 & 0.1162 & 0.00 \\
\hline Rad. data $Y_{P O Z R F N}$ & 0.0196 & 0.0040 & 0.08 & 0.0294 & 0.0052 \\
\hline \multicolumn{6}{|l|}{ Corrections due to } \\
\hline TOF window cut & 1.0010 & 1.0025 & 1.0073 & 1.0168 & 1.0133 \\
\hline EDT Correction & 1.0405 & 1.0213 & 1.0497 & 1.0379 & 1.0313 \\
\hline VDC Corr. Factor & 1.6062 & 1.5619 & 1.6550 & 1.5817 & 1.4711 \\
\hline A1 corr. factor & 1.019 & 1.019 & 1.019 & 1.019 & 1.019 \\
\hline A2 corr. factor & 1.0180 & 1.0180 & 1.0180 & 1.02 & 1.02 \\
\hline Luminosity $\left(n b^{-1}\right)$ & $8.3883 \mathrm{e}+09$ & $4.1824 \mathrm{e} 10$ & $9.3485 \mathrm{e} 11$ & $+9.61 \mathrm{E}+09$ & $4.26 \mathrm{E}+06$ \\
\hline Kaon Absorbtion & 1.0463 & 1.0463 & 1.0463 & 1.0443 & 1.0443 \\
\hline Gas Cerenkov & 1.0227 & 1.0227 & 1.0227 & 1.0227 & 1.0227 \\
\hline Target Boiling & 1.025 & 1.08 & 1.06 & 1.0500 & 1.0800 \\
\hline Corrected Yield $(\mathrm{Hz})$ & 0.0373 & 0.0078 & 0.0016 & 0.0562 & 0.0093 \\
\hline$\frac{d \sigma}{d \Omega}(\mathrm{nb} / \mathrm{sr})$ & 150.0800 & 135.3595 & 129.1338 & 147.4046 & 135.1342 \\
\hline Data/Mceep & 0.9884 & 0.9607 & 1.0212 & 0.9909 & 0.9962 \\
\hline
\end{tabular}

Table 11: Unseparated cross sections and corresponding correction factors: Kin 2, 24, 18, 3 and 25 


\begin{tabular}{|c|c|c|c|c|c|}
\hline Kin. no. & KIN 4 & KIN 22 & KIN 11 & KIN 26 & KIN 19 \\
\hline \multicolumn{6}{|l|}{ Mceep rad. } \\
\hline$d^{6} \sigma\left(\frac{f m^{2}}{M \rho V^{2} s r^{2}}\right)$ & $1.950 \mathrm{E}-13$ & $5.732 \mathrm{E}-14$ & $3.028 \mathrm{E}-13$ & $1.087 \mathrm{E}-13$ & $5.901 \mathrm{E}-14$ \\
\hline Yield $(\mathrm{Hz})$ & 0.0666 & 0.0081 & 0.0797 & 0.0197 & 0.0040 \\
\hline \multicolumn{6}{|l|}{ Mceep no rad. } \\
\hline$d^{5} \sigma\left(\frac{f m^{2}}{M e V s r^{2}}\right)$ & $2.586 \mathrm{E}-11$ & $+7.364 \mathrm{E}-12$ & $4.216 \mathrm{E}-11$ & $1.450 \mathrm{E}-11$ & $5.290 \mathrm{E}-12$ \\
\hline Yield (Hz) & 0.0724 & 0.0086 & 0.0869 & 0.0209 & 0.0045 \\
\hline Rad. Correction & 1.0864 & 1.0612 & 1.0900 & 1.0619 & 1.1212 \\
\hline \multicolumn{6}{|l|}{ KFIT2 } \\
\hline$d^{5} \sigma \frac{f m^{2}}{M e V s r^{2}}$ & $2.666 \mathrm{E}-11$ & $+7.551 \mathrm{E}-05$ & $2.607 \mathrm{E}-10$ & $1.501 \mathrm{E}-11$ & $5.421 \mathrm{E}-12$ \\
\hline$\frac{d \sigma}{d \Omega}(\mathrm{nb} / \mathrm{sr}) \mathrm{kfit} 2$ & 141.3173 & 126.8036 & 187.1379 & 174.8182 & 159.1480 \\
\hline Survival fraction kfit & 0.2701 & 0.2701 & 0.1719 & 0.1719 & 0.1719 \\
\hline Photon Flux * Jacob & $6.984 \mathrm{E}-13$ & $2.205 \mathrm{E}-06$ & $+8.103 \mathrm{E}-12$ & $4.994 \mathrm{E}-13$ & $1.981 \mathrm{E}-13$ \\
\hline Yield NO RFN cut & & & & 2812.80 & \\
\hline Yield RFN +0.008 & 1539.40 & 580.40 & 842.10 & 727.40 & 455.50 \\
\hline Eq. time at $75 \mu \mathrm{A}$ & $4.30 \mathrm{E}+04$ & $1.33 \mathrm{E}+05$ & $2.10 \mathrm{E}+04$ & $6.66 \mathrm{E}+04$ & $2.21 \mathrm{E}+05$ \\
\hline Rad. data $Y_{N O R F N}$ & 0.00 & 0.00 & 0.00 & 0.0422 & 0.00 \\
\hline Rad. data $Y_{P O Z R F N}$ & 0.0358 & 0.0044 & 0.0401 & 0.0109 & 0.0021 \\
\hline \multicolumn{6}{|l|}{ Corrections due to } \\
\hline TOF window cut & 1.0148 & 1.0132 & 1.0248 & 1.0132 & 1.0132 \\
\hline EDT Correction & 1.0289 & 1.0279 & 1.1079 & 1.0193 & 1.0432 \\
\hline VDC Corr. Factor & 1.4890 & 1.4281 & 1.4006 & 1.4706 & 1.4889 \\
\hline A1 corr. factor & 1.019 & 1.019 & 1.019 & 1.019 & 1.019 \\
\hline A2 corr. factor & 1.0080 & 1.0080 & 1.0270 & 1.0017 & 1.0017 \\
\hline Luminosity $\left(n b^{-1}\right)$ & $1.33 \mathrm{E} 10$ & $1.23 \mathrm{E}+05$ & $+8.67 \mathrm{E} 52$ & $2.06 \mathrm{E} 10$ & $6.85 \mathrm{E} 10$ \\
\hline Kaon Absorbtion & 1.0440 & 1.0440 & 1.0443 & 1.0463 & 1.0463 \\
\hline Gas Cerenkov & 1.0227 & 1.0227 & 1.0227 & 1.0227 & 1.0227 \\
\hline Target Boiling & 1.0600 & 1.0600 & 1.0600 & 1.0600 & 1.0600 \\
\hline Corrected Yield (Hz) & 0.0647 & 0.0076 & 0.0756 & 0.0192 & 0.0038 \\
\hline$\frac{d \sigma}{d \Omega}{ }_{\Lambda}(\mathrm{nb} / \mathrm{sr})$ & 137.1512 & 118.1339 & 177.5673 & 170.6966 & 149.6765 \\
\hline Data/Mceep & 0.9705 & 0.9316 & 0.9489 & 0.9764 & 0.9405 \\
\hline
\end{tabular}

Table 12: Unseparated cross sections and corresponding correction factors: Kin 4, 22, 11, 26 and 19 


\begin{tabular}{|c|c|c|c|c|c|}
\hline Kin. no. & KIN 5 & KIN 27 & KIN 17 & KIN 12 & KIN 28 \\
\hline \multicolumn{6}{|l|}{ Mceep rad. } \\
\hline$d^{6} \sigma\left(\frac{f m^{2}}{M e V^{2} s r^{2}}\right)$ & $0.29838 \mathrm{E}-12$ & $1.022 \mathrm{E}-13$ & $5.263 \mathrm{E}-14$ & $3.239 \mathrm{E}-13$ & $1.013 \mathrm{E}-13$ \\
\hline Yield $(\mathrm{Hz})$ & 0.0841 & 0.0193 & 0.0038 & 0.0995 & 0.0196 \\
\hline \multicolumn{6}{|l|}{ Mceep no rad. } \\
\hline$d^{5} \sigma\left(\frac{f m^{2}}{M e V s r^{2}}\right)$ & $4.045 \mathrm{E}-11$ & $1.433 \mathrm{E}-11$ & $4.980 \mathrm{E}-12$ & $4.455 \mathrm{E}-11$ & $1.396 \mathrm{E}-11$ \\
\hline Yield $(\mathrm{Hz})$ & 0.0909 & 0.0202 & 0.0041 & 0.1078 & 0.0205 \\
\hline Rad. Correction & 1.0803 & 1.0489 & 1.0911 & 1.0836 & 1.0451 \\
\hline \multicolumn{6}{|l|}{ KFIT2 } \\
\hline$d^{5} \sigma \frac{f m^{2}}{M r V V s r^{2}}$ & $4.225 \mathrm{E}-11$ & $1.497 \mathrm{E}-11$ & $5.178 \mathrm{E}-12$ & $4.661 \mathrm{E}-11$ & $1.454 \mathrm{E}-11$ \\
\hline$\frac{d \sigma}{d \Omega}(\mathrm{nb} / \mathrm{sr}) \mathrm{kfit} 2$ & 185.1794 & 172.9277 & 156.4907 & 182.0988 & 168.0083 \\
\hline Survival fraction kfit & 0.1871 & 0.1871 & 0.1871 & 0.2123 & 0.2123 \\
\hline Photon Flux * Jacob & $1.219 \mathrm{E}-12$ & $4.627 \mathrm{E}-13$ & $1.768 \mathrm{E}-13$ & $1.206 \mathrm{E}-12$ & $4.076 \mathrm{E}-13$ \\
\hline \multicolumn{6}{|l|}{ Yield NO RFN cut } \\
\hline Yield RFN +0.008 & 1413.60 & 769.40 & 376.70 & 1198.40 & 1346.20 \\
\hline Eq. time at $75 \mu \mathrm{A}$ & $3.06 \mathrm{E}+04$ & $6.61 \mathrm{E}+04$ & $1.84 \mathrm{E}+05$ & $2.19 \mathrm{E}+04$ & $1.29 \mathrm{E}+05$ \\
\hline Rad. data $Y_{N O R F N}$ & 0.00 & 0.00 & 0.00 & 0.00 & 0.00 \\
\hline Rad. data $Y_{P O Z R F N}$ & 0.0463 & 0.0116 & 0.0020 & 0.0547 & 0.0104 \\
\hline \multicolumn{6}{|l|}{ Corrections due to } \\
\hline TOF window cut & 1.0345 & 1.0026 & 1.0073 & 1.0248 & 1.0470 \\
\hline EDT Correction & 1.0360 & 1.0087 & 1.0469 & 1.0328 & 1.0158 \\
\hline VDC Corr. Factor & 1.4247 & 1.4259 & 1.5023 & 1.3537 & 1.4318 \\
\hline A1 corr. factor & 1.019 & 1.019 & 1.019 & 1.019 & 1.019 \\
\hline A2 corr. factor & 1.0051 & 1.0051 & 1.0051 & 1.0467 & 1.0467 \\
\hline Luminosity $\left(n b^{-1}\right)$ & $+9.45 \mathrm{E}+09$ & $2.04 \mathrm{E} 10$ & $5.68 \mathrm{E} 10$ & $1.31 \mathrm{E}+05$ & $3.99 \mathrm{E} 10$ \\
\hline Kaon Absorbtion & 1.0452 & 1.0452 & 1.0452 & 1.0452 & 1.0452 \\
\hline Gas Cerenkov & 1.0227 & 1.0227 & 1.0227 & 1.0227 & 1.0227 \\
\hline Target Boiling & 1.0500 & 1.0300 & 1.0500 & 1.0500 & 1.0500 \\
\hline Corrected Yield $(\mathrm{Hz})$ & 0.0812 & 0.0189 & 0.0037 & 0.0939 & 0.0190 \\
\hline$\frac{d \sigma}{d \Omega}(\mathrm{nb} / \mathrm{sr})$ & 178.8543 & 169.9561 & 154.3913 & 171.7756 & 162.7082 \\
\hline Data/Mceep & 0.9658 & 0.9828 & 0.9866 & 0.9433 & 0.9685 \\
\hline
\end{tabular}

Table 13: Unseparated cross sections and corresponding correction factors: Kin 5, 27, 17, 12 and 28 


\begin{tabular}{|c|c|}
\hline Kin. no. & KIN 1 Prime \\
\hline \multicolumn{2}{|l|}{ Mceep rad. } \\
\hline$d^{6} \sigma\left(\frac{f m^{2}}{M f e V^{2} s \tau^{2}}\right)$ & $1.5865 \mathrm{e}-13$ \\
\hline Yield $(\mathrm{Hz})$ & 0.024727 \\
\hline \multicolumn{2}{|l|}{ Mceep no rad. } \\
\hline$d^{5} \sigma\left(\frac{f m^{2}}{M e V s r^{2}}\right)$ & $2.5201 \mathrm{e}-11$ \\
\hline Yield $(\mathrm{Hz})$ & 0.02649 \\
\hline Rad. Correction & 1.07130 \\
\hline \multicolumn{2}{|l|}{ KFIT2 } \\
\hline$d^{5} \sigma \frac{f m^{2}}{M e V s r^{2}}$ & $2.7027 \mathrm{E}-11$ \\
\hline$\frac{d \sigma}{d \Omega}(\mathrm{nb} / \mathrm{sr}) \mathrm{kfit} 2$ & 149.41765 \\
\hline Survival fraction kfit & 0.14780 \\
\hline Photon Flux * Jacob & $1.2238 \mathrm{E}-12$ \\
\hline Yield NO RFN cut & 1723.0 \\
\hline Yield RFN +0.008 & 485.40 \\
\hline Eq. time at $75 \mu \mathrm{A}$ & $4.38 \mathrm{E}+04$ \\
\hline Rad. data $Y_{N O R F N}$ & 0.03935 \\
\hline Rad. data $Y_{P O Z R F N}$ & 0.01108 \\
\hline \multicolumn{2}{|l|}{ Corrections due to } \\
\hline TOF window cut & 1.00980 \\
\hline EDT Correction & 1.04000 \\
\hline VDC Corr. Factor & 1.53559 \\
\hline A1 corr. factor & 1.01913 \\
\hline A2 corr. factor & 1.02242 \\
\hline Luminosity $\left(n b^{-1}\right)$ & $1.3542 \mathrm{E}+10$ \\
\hline Kaon Absorbtion & 1.04661 \\
\hline Gas Cerenkov & 1.02785 \\
\hline Target Boiling & 1.03800 \\
\hline Corrected Yield $(\mathrm{Hz})$ & 0.02080 \\
\hline$\frac{d \sigma}{d \Omega}(\mathrm{nb} / \mathrm{sr})$ & 125.680 \\
\hline Data/Mceep & 0.84113 \\
\hline
\end{tabular}

Table 14: Unseparated cross sections and corresponding correction factors: Kin 1 Prime, 2002 
In the tables 10 to 14 the quantities TOF window cut and EDT Correction are calculated using the following formulas:

$$
\text { TOF window cut }=(2 \cdot \operatorname{Normdist}(3 \cdot \sigma)-1)^{-1}
$$

where $\sigma$ is the standard deviation as obtained from fitting the $K$ peak in the corrected time of flight spectra, and Normdist is the cumulative distribution function for the gaussian (normal) distribution, which is calculated numerically in a spreadsheet [GNU03].

$$
\text { EDT Correction }=\left[1-\frac{\sum_{i=1 \text { st run }}^{\text {Last run }} E D T_{i} \cdot Q_{i}}{\sum_{i=1 \text { st run }}^{\text {Last run }} Q_{i}}\right]^{-1}
$$

where $E D T_{i}$ is the electronic dead time for run $i$ and $Q_{i}$ is the charge of run $i$. The electronic deadtime was also weighted by the runs' charge for all kinematics (the same procedure was applied for $V D C$ efficiency see Section 3.5). All other corrections factors are explained in Section 3. 
B E98-108 experiment: $\Lambda$ and $\Sigma$ missing mass spectra for all kinematics with the R-function cut applied after background subtraction (see Section 4.3)

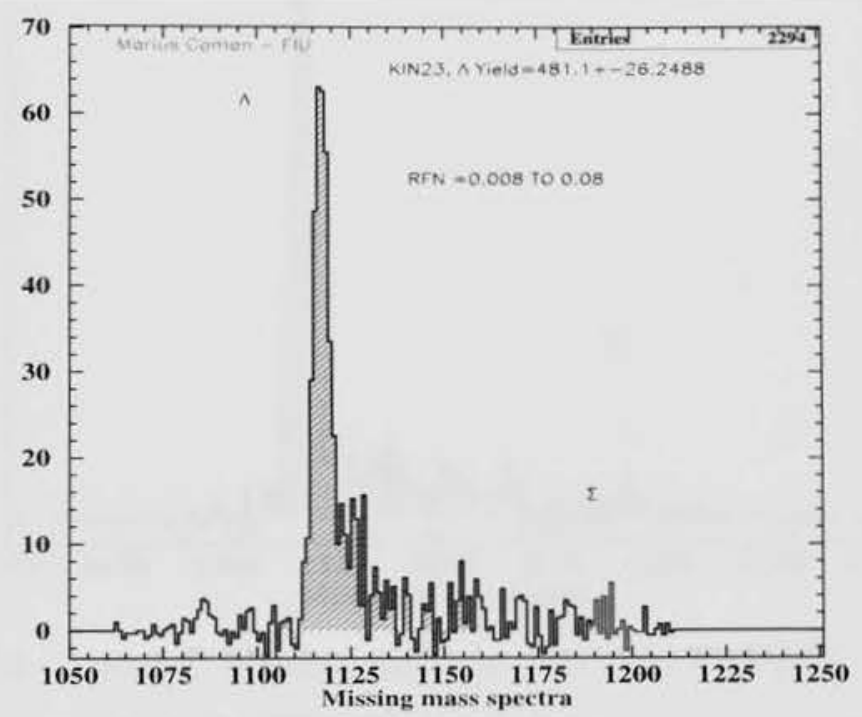

Figure 87: $\Lambda$ and $\Sigma$ Missing mass $\left(x\right.$ axis is in $\left.\mathrm{MeV} / \mathrm{c}^{2}\right)$, Kin. 23.

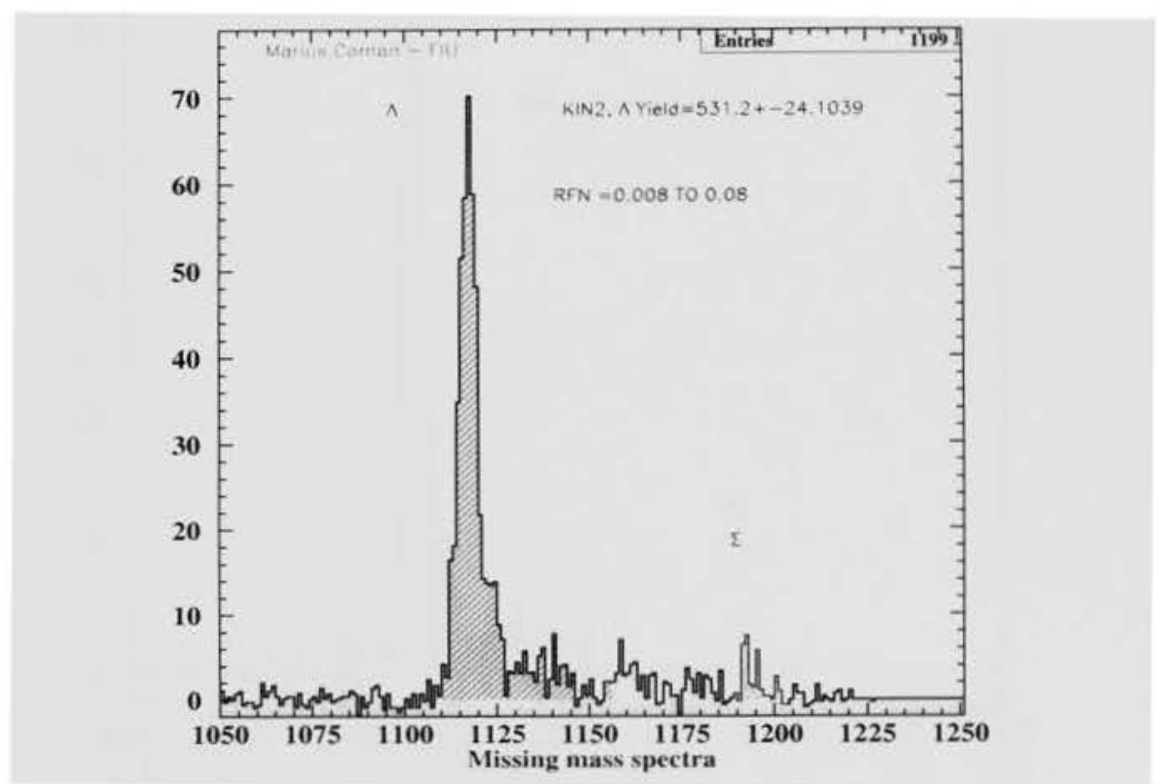

Figure 88: $\Lambda$ and $\Sigma$ Missing mass $\left(x\right.$ axis is in $\left.M e V / c^{2}\right)$, Kin. 2 . 


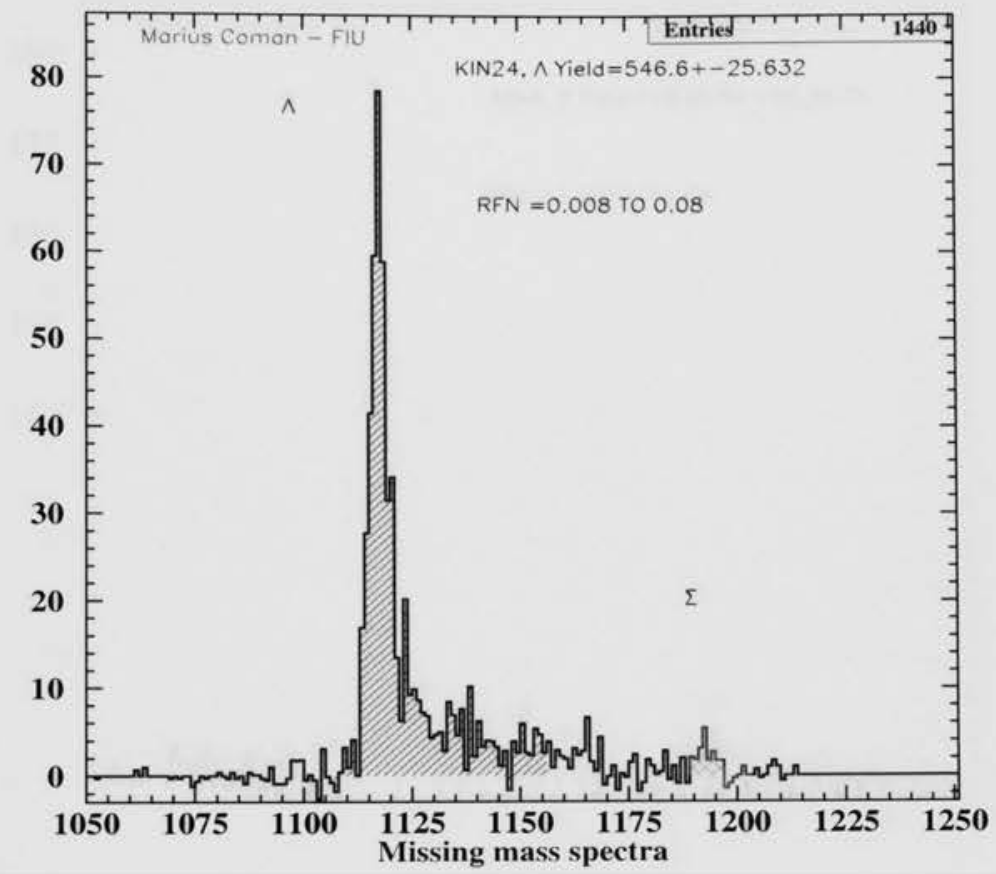

Figure 89: $\Lambda$ and $\Sigma$ Missing mass ( $x$ axis is in $\mathrm{MeV} / \mathrm{c}^{2}$ ), Kin. 24 .

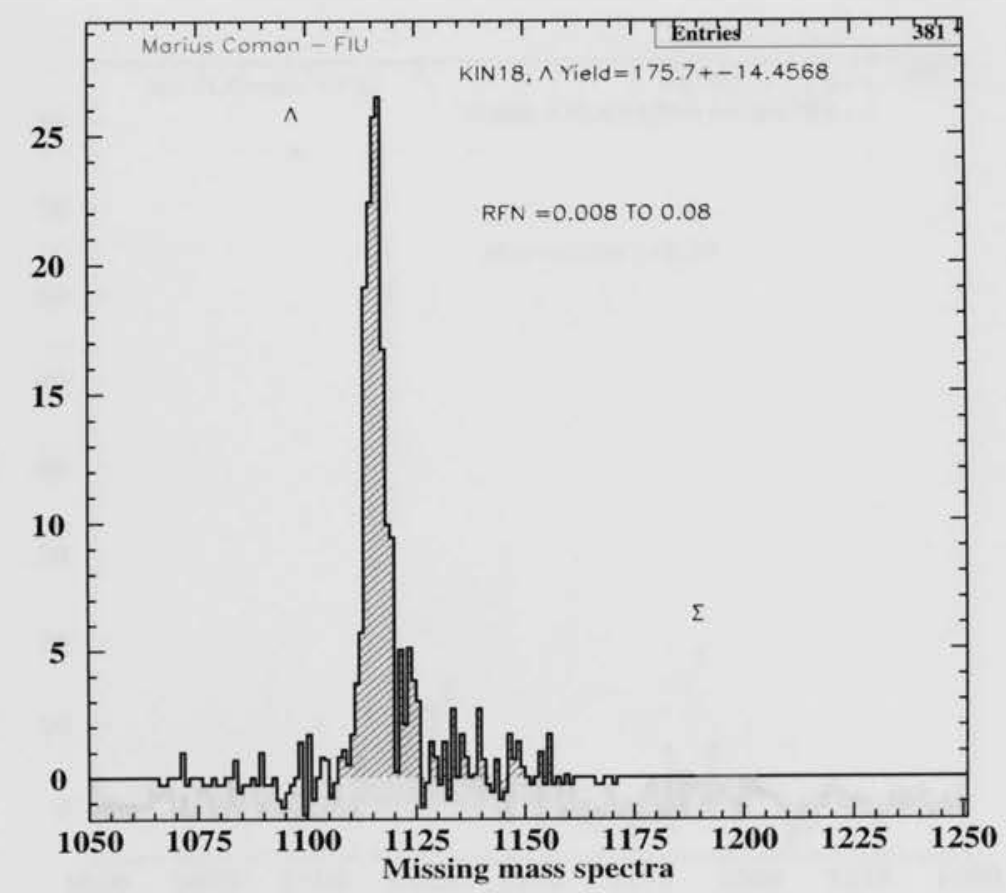

Figure 90: $\Lambda$ and $\Sigma$ Missing mass ( $x$ axis is in $M e V / c^{2}$ ), Kin. 18 . 


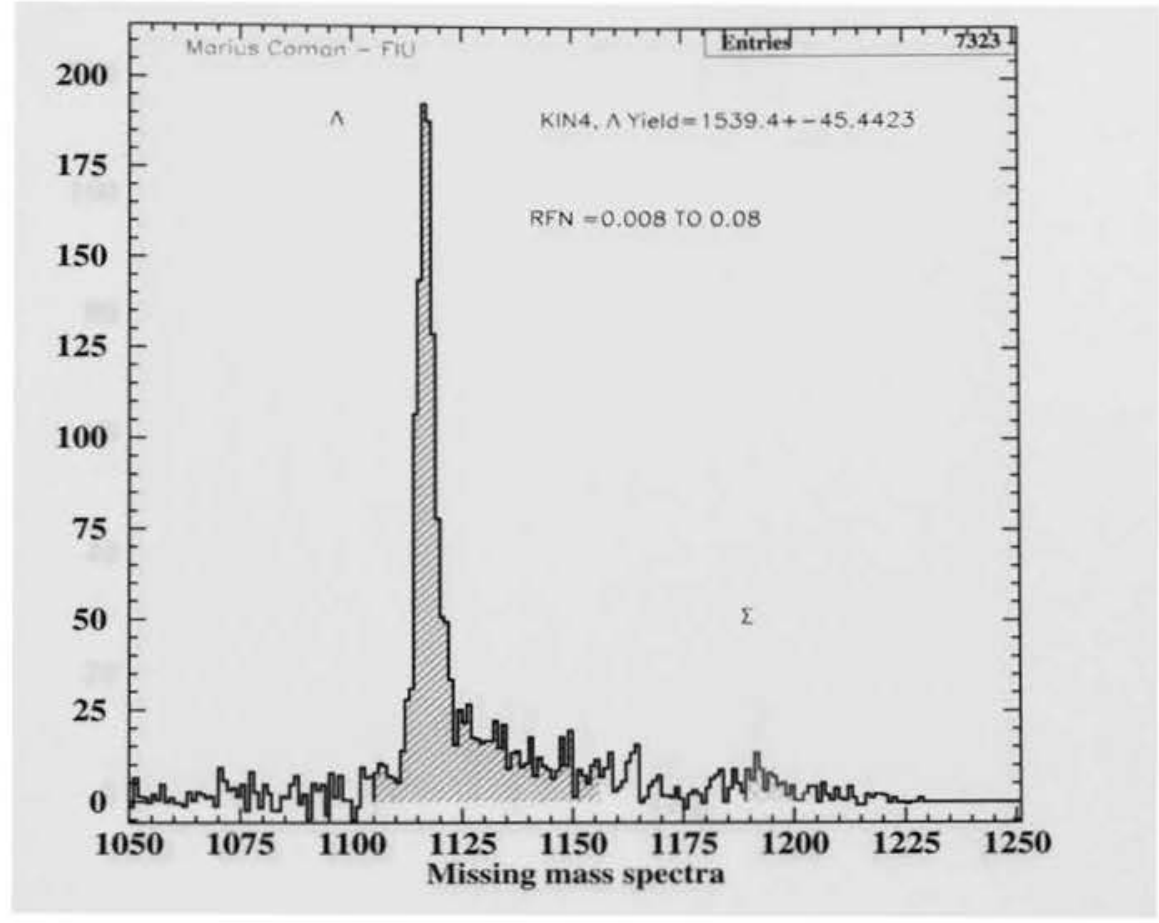

Figure 91: $\Lambda$ and $\Sigma$ Missing mass ( $x$ axis is in $\mathrm{MeV} / \mathrm{c}^{2}$ ), Kin. 4 .

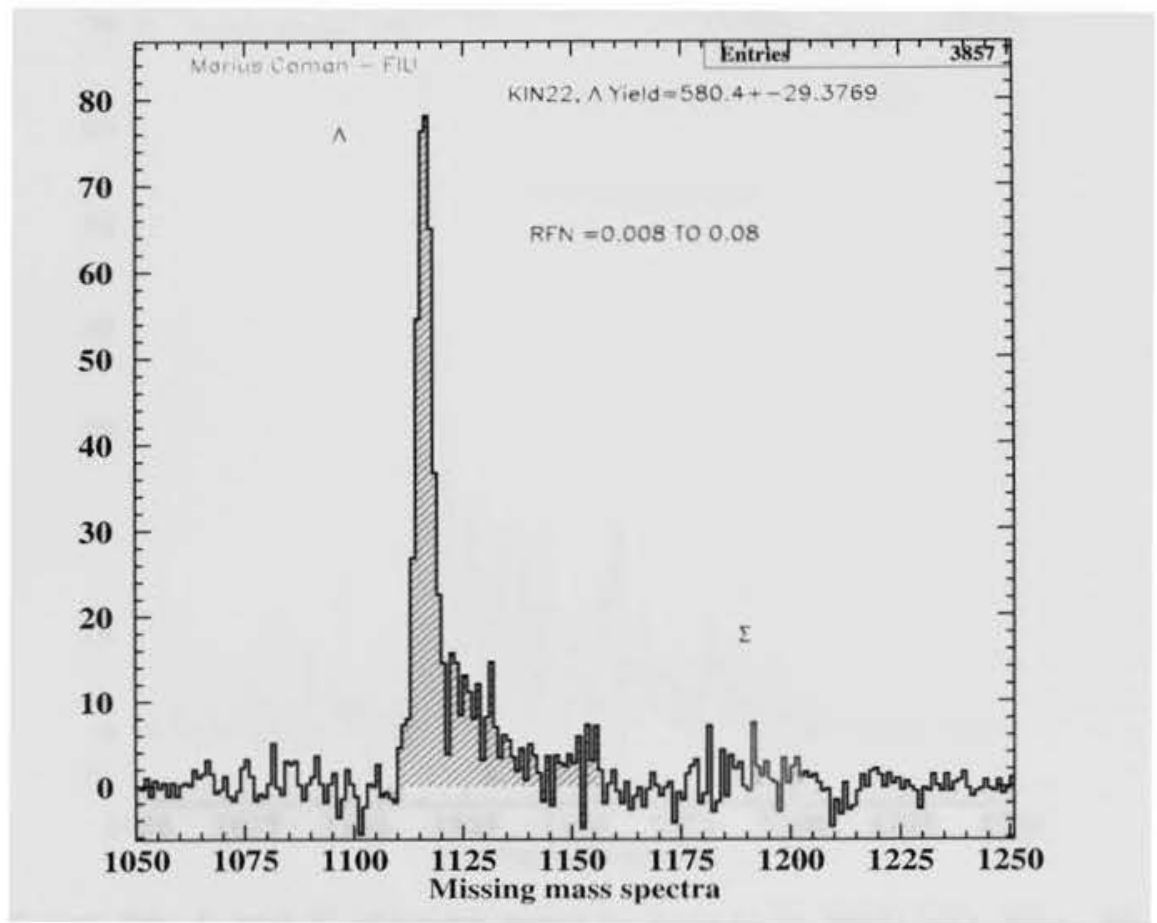

Figure 92: $\Lambda$ and $\Sigma$ Missing mass $\left(x\right.$ axis is in $\left.\mathrm{MeV} / \mathrm{c}^{2}\right)$, Kin. 22 . 


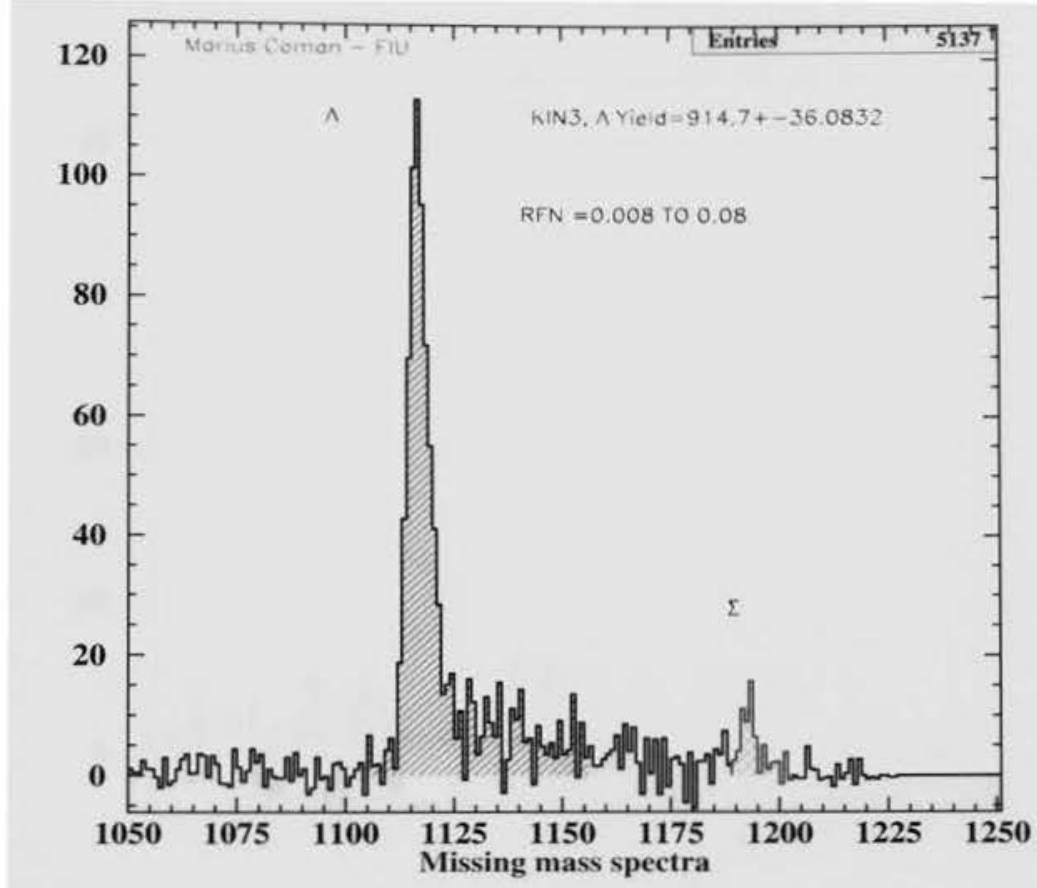

Figure 93: $\Lambda$ and $\Sigma$ Missing mass $\left(x\right.$ axis is in $\left.\mathrm{MeV} / \mathrm{c}^{2}\right)$, Kin. 3 .

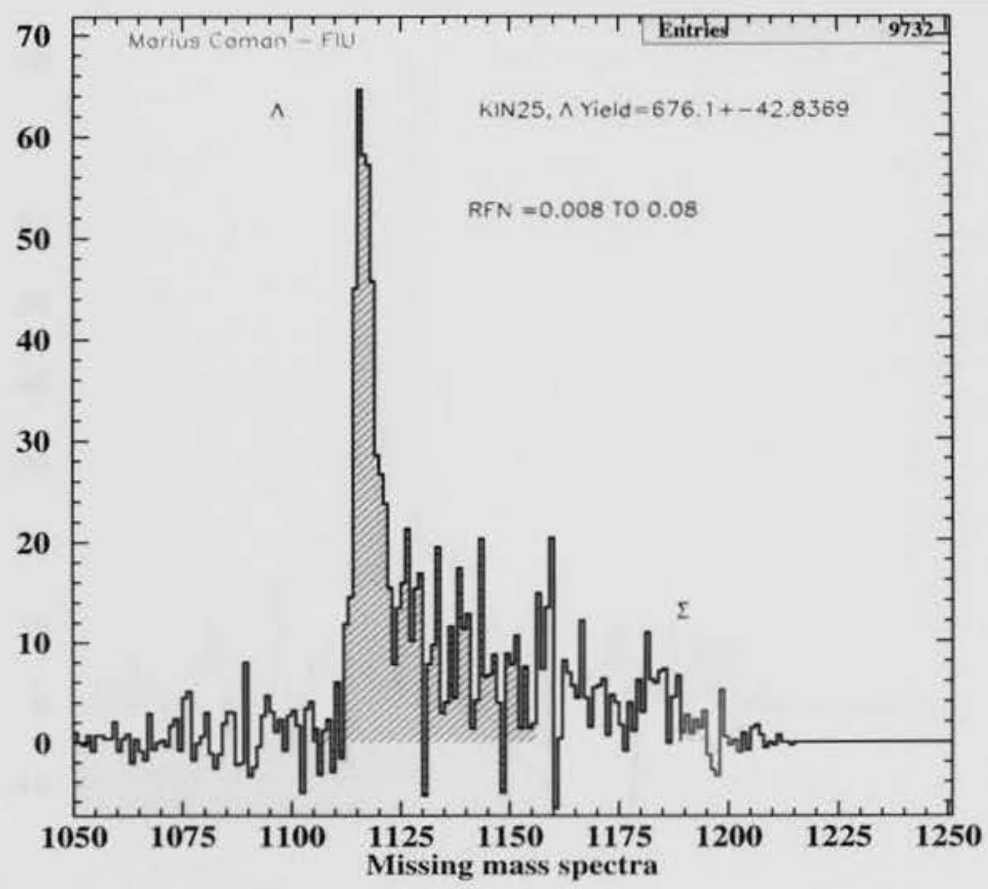

Figure 94: $\Lambda$ and $\Sigma$ Missing mass $\left(x\right.$ axis is in $\left.\mathrm{MeV} / \mathrm{c}^{2}\right)$, Kin. 25 . 


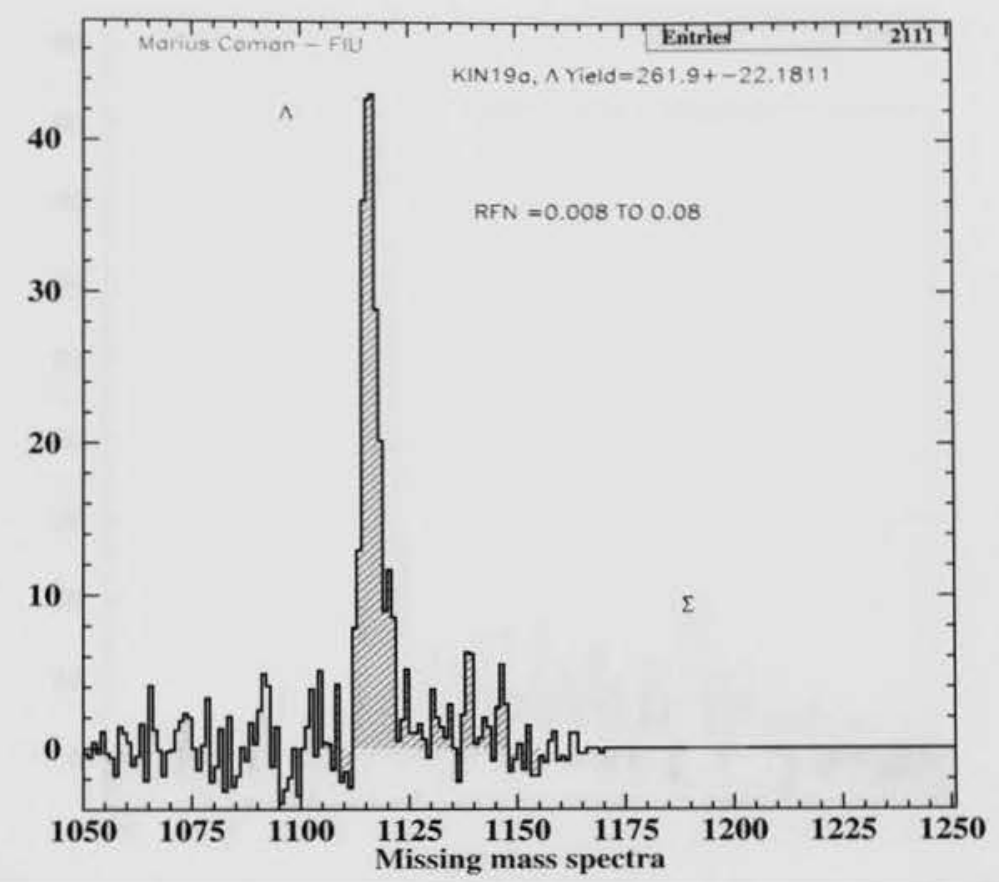

Figure 95: $\Lambda$ and $\Sigma$ Missing mass ( $x$ axis is in $\mathrm{MeV} / \mathrm{c}^{2}$ ), Kin. $19 \mathrm{~A}$.

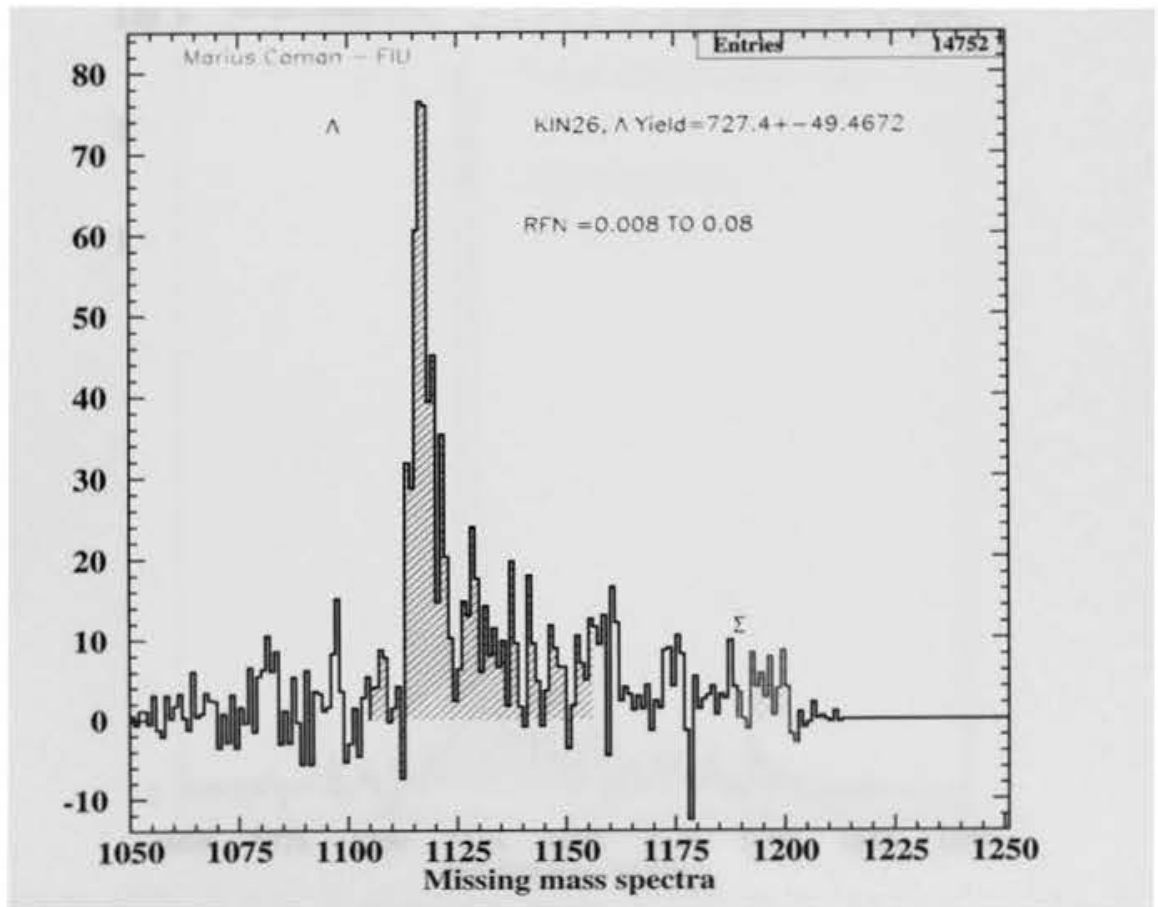

Figure 96: $\Lambda$ and $\Sigma$ Missing mass ( $x$ axis is in $\mathrm{MeV} / \mathrm{c}^{2}$ ), Kin. 26 . 


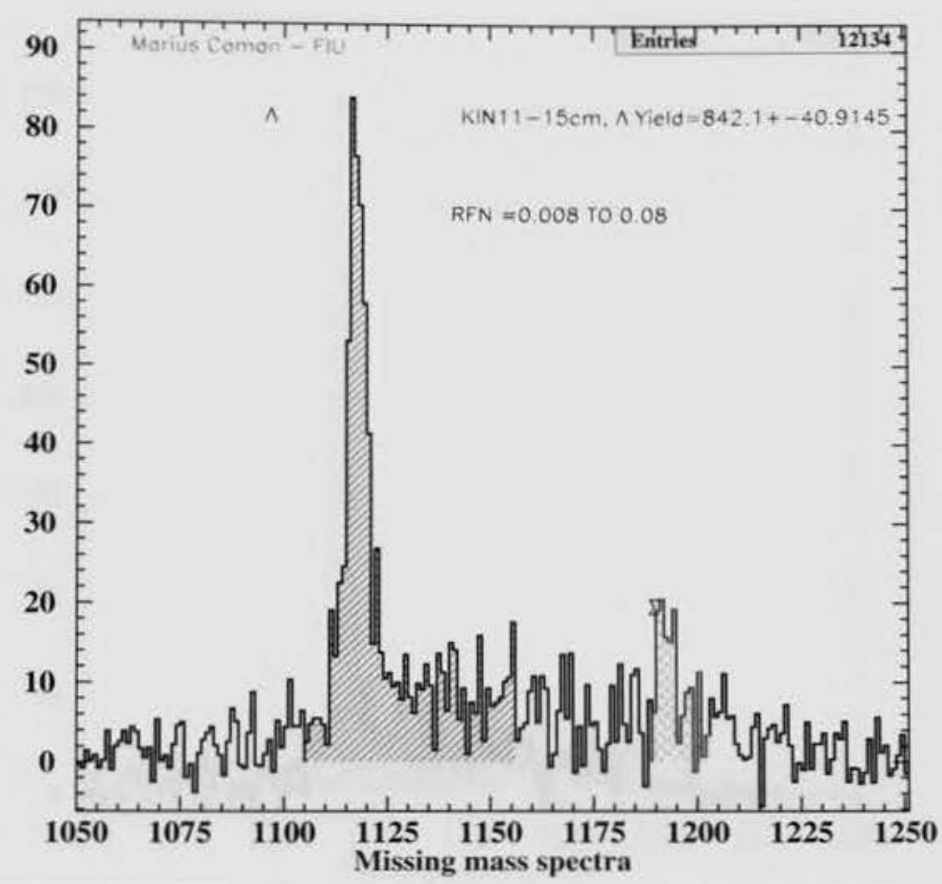

Figure 97: $\Lambda$ and $\Sigma$ Missing mass $\left(x\right.$ axis is in $\left.\mathrm{MeV} / \mathrm{c}^{2}\right)$, Kin. 11 .

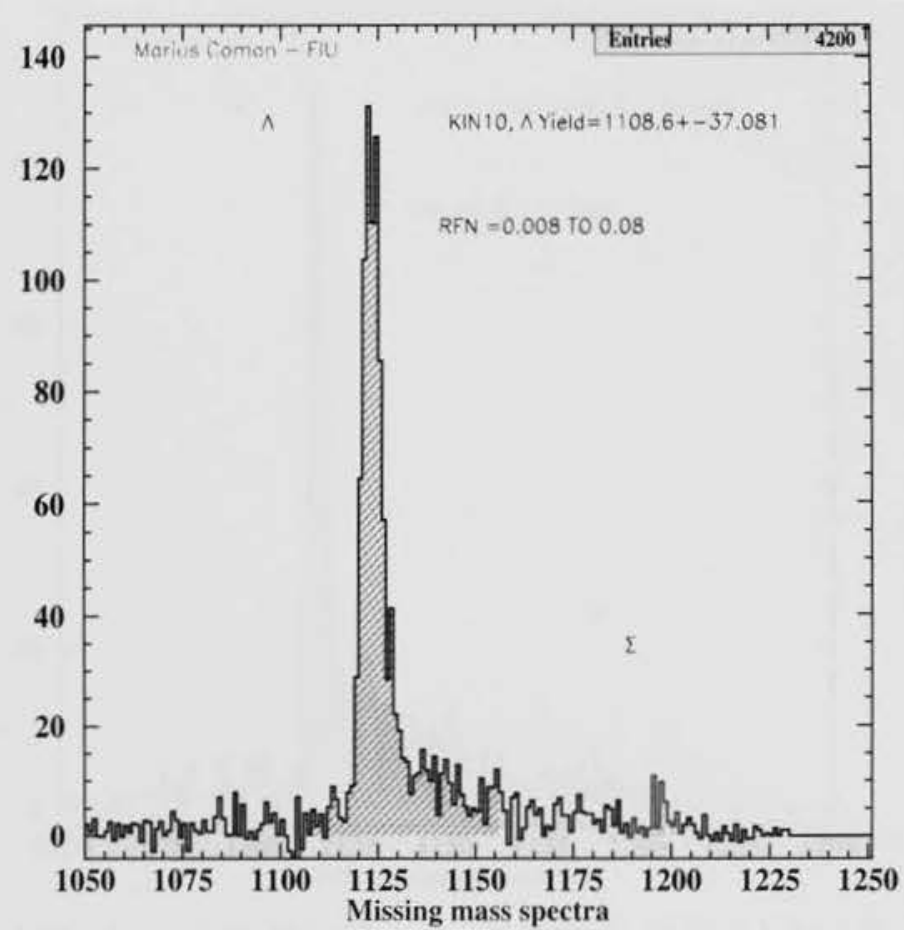

Figure 98: $\Lambda$ and $\Sigma$ Missing mass $\left(x\right.$ axis is in $\left.M e V / c^{2}\right)$, Kin. 10 . 


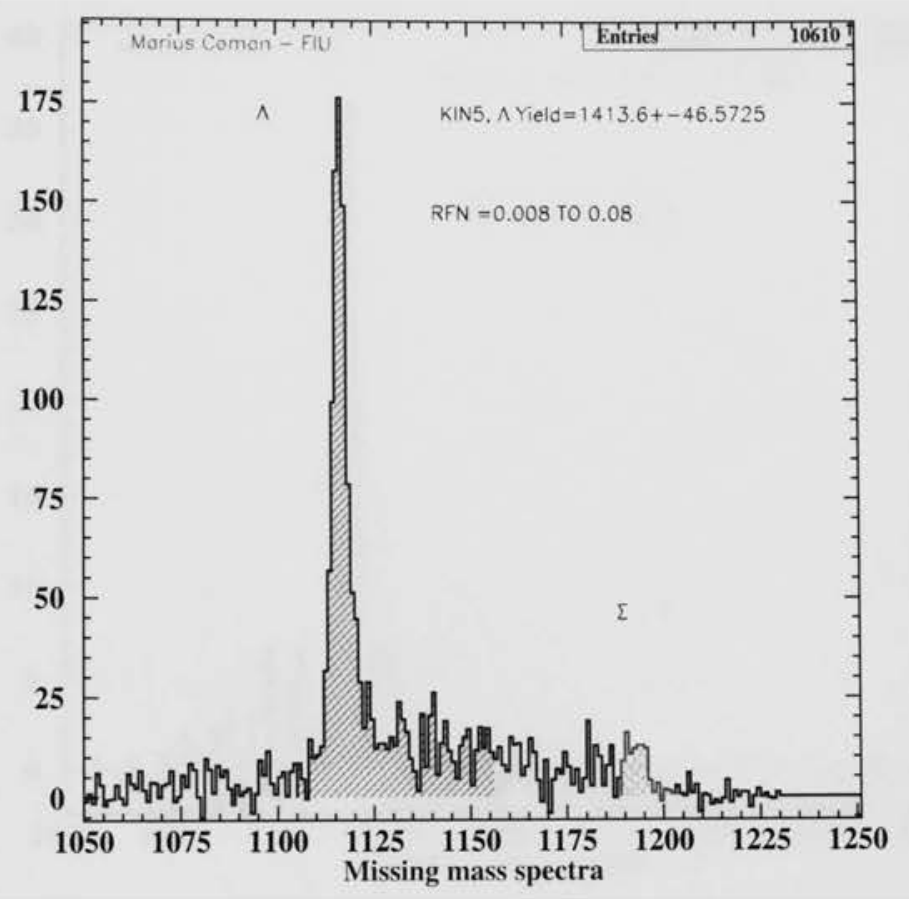

Figure 99: $\Lambda$ and $\Sigma$ Missing mass $\left(x\right.$ axis is in $\left.\mathrm{MeV} / \mathrm{c}^{2}\right)$, Kin. 5 .

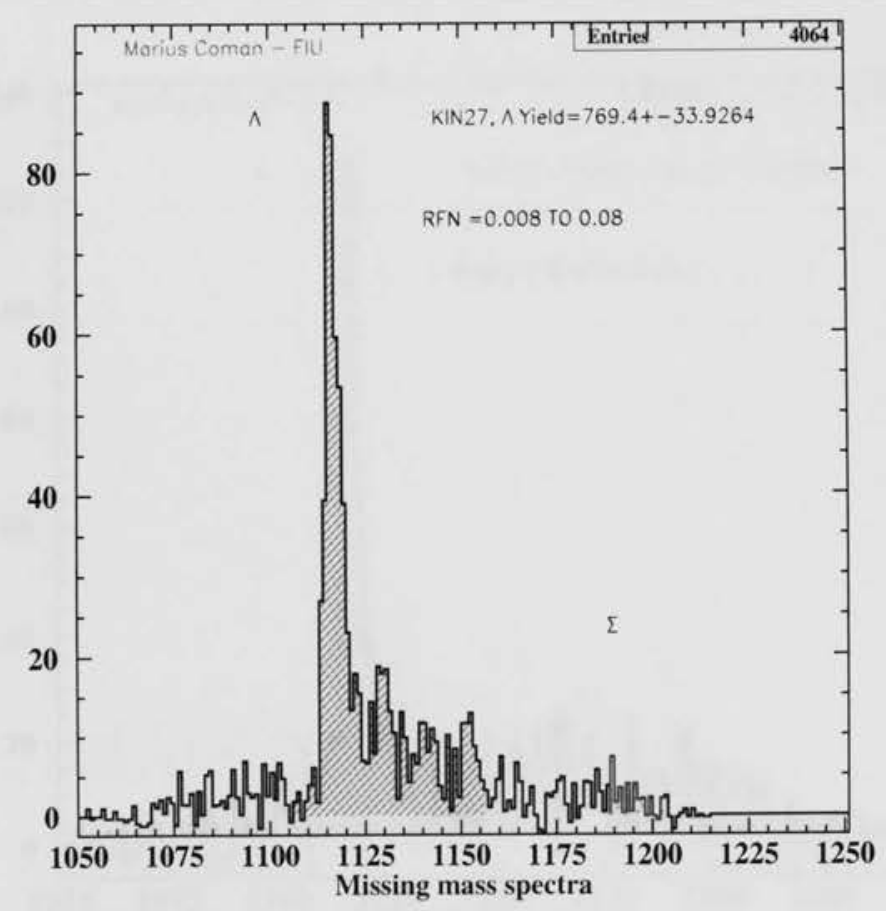

Figure 100: $\Lambda$ and $\Sigma$ Missing mass ( $x$ axis is in $\mathrm{MeV} / \mathrm{c}^{2}$ ), Kin. 27. 


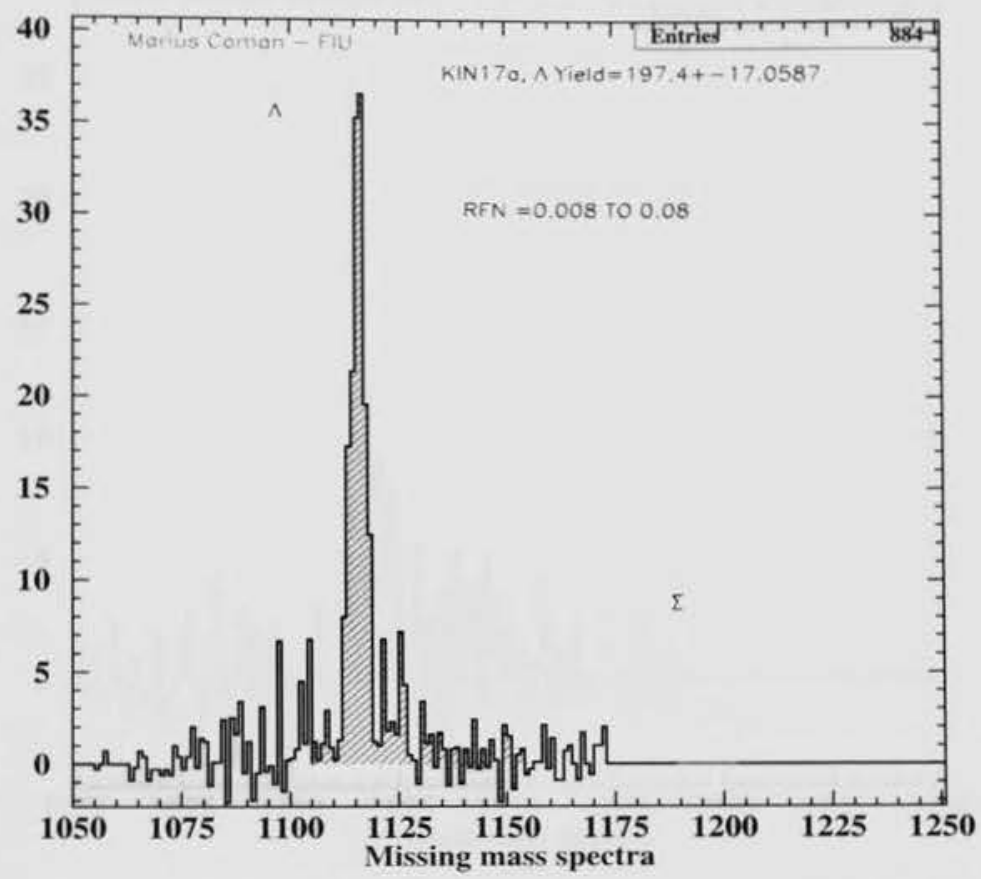

Figure 101: $\Lambda$ and $\Sigma$ Missing mass ( $x$ axis is in $\mathrm{MeV} / \mathrm{c}^{2}$ ), Kin. $17 \mathrm{~A}$.

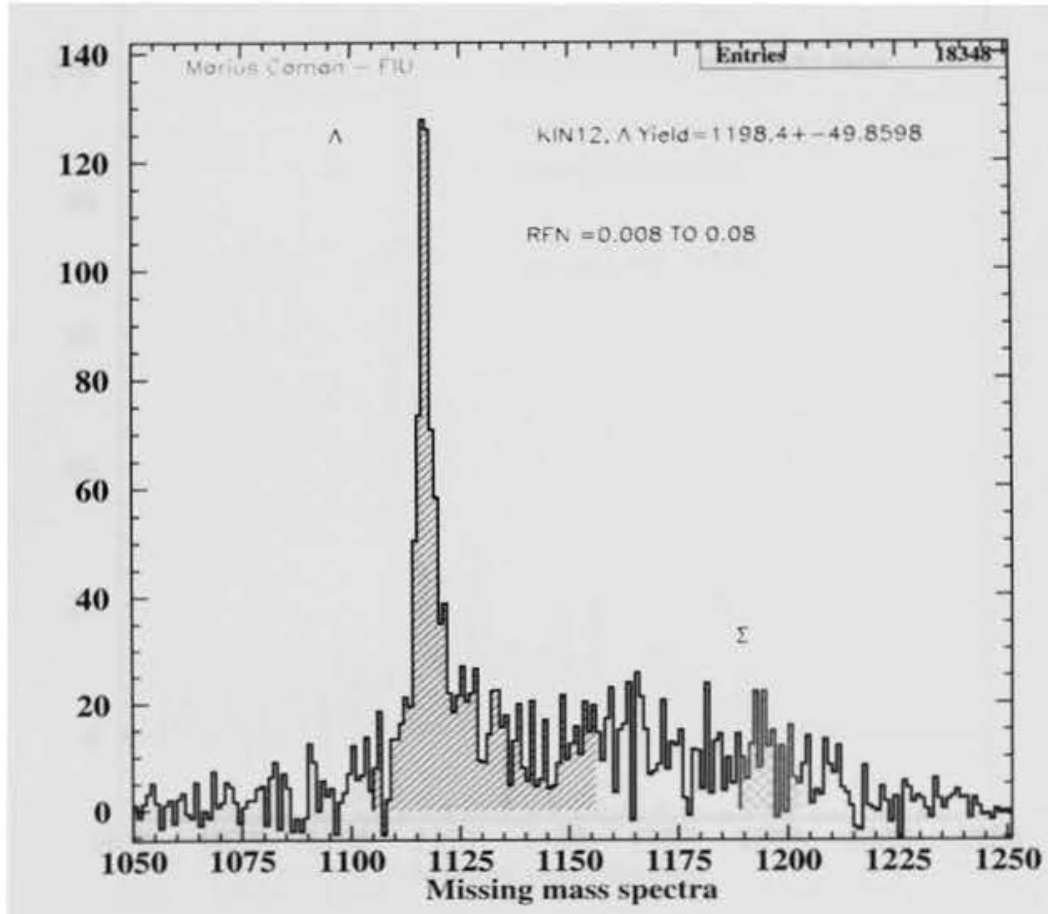

Figure 102: $\Lambda$ and $\Sigma$ Missing mass ( $x$ axis is in $\mathrm{MeV} / \mathrm{c}^{2}$ ), Kin. 12 . 


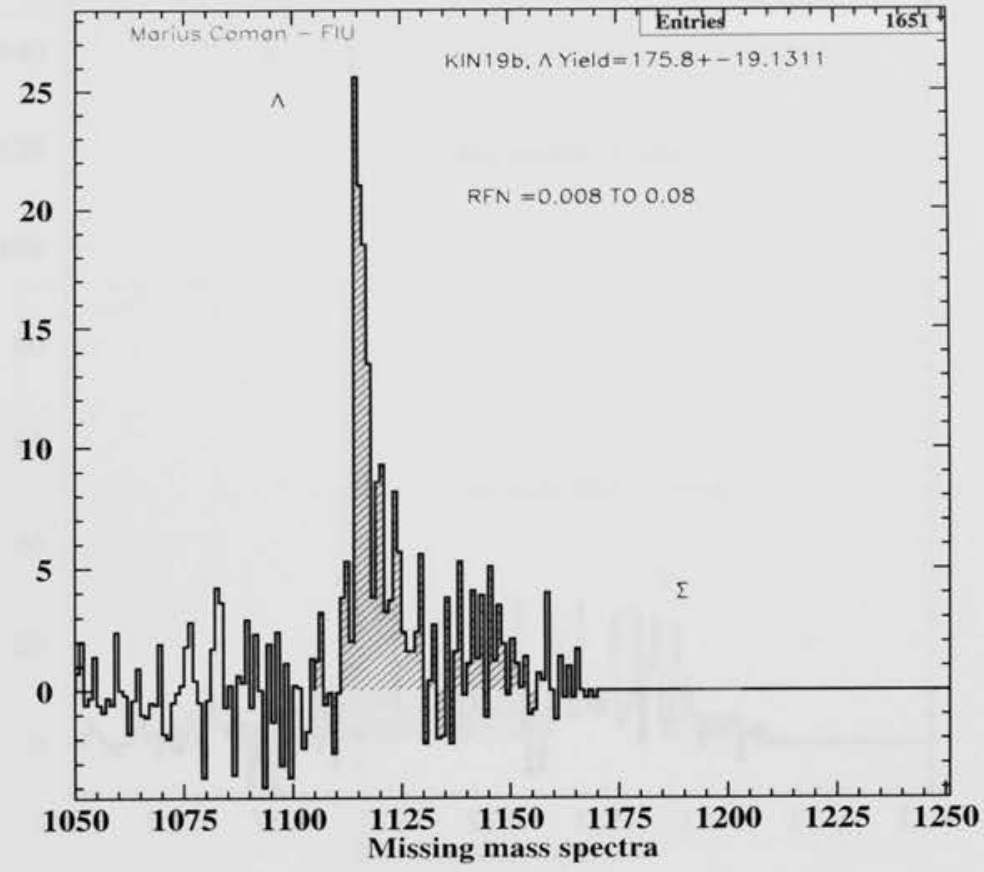

Figure 103: $\Lambda$ and $\Sigma$ Missing mass $\left(x\right.$ axis is in $\left.\mathrm{MeV} / \mathrm{c}^{2}\right)$, Kin. $19 \mathrm{~B}$

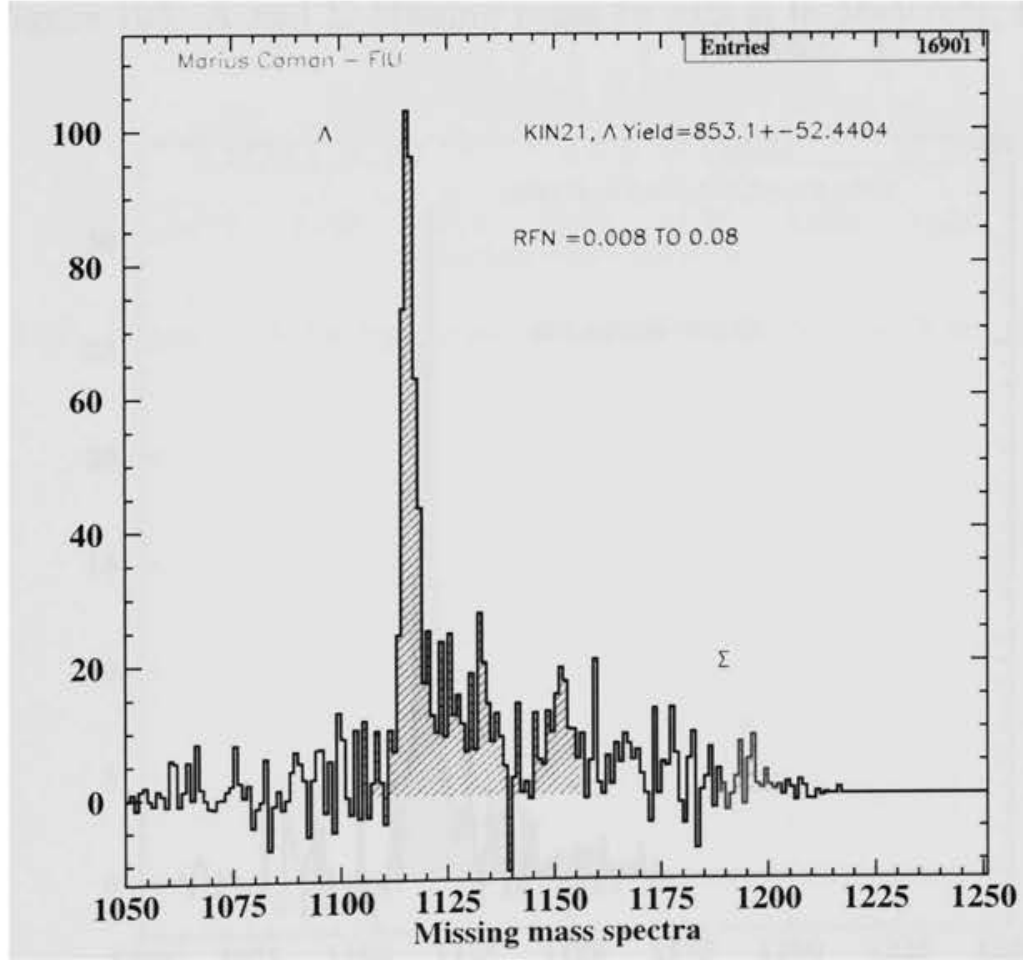

Figure 104: $\Lambda$ and $\Sigma$ Missing mass ( $x$ axis is in $\mathrm{MeV} / \mathrm{c}^{2}$ ), Kin. 21 


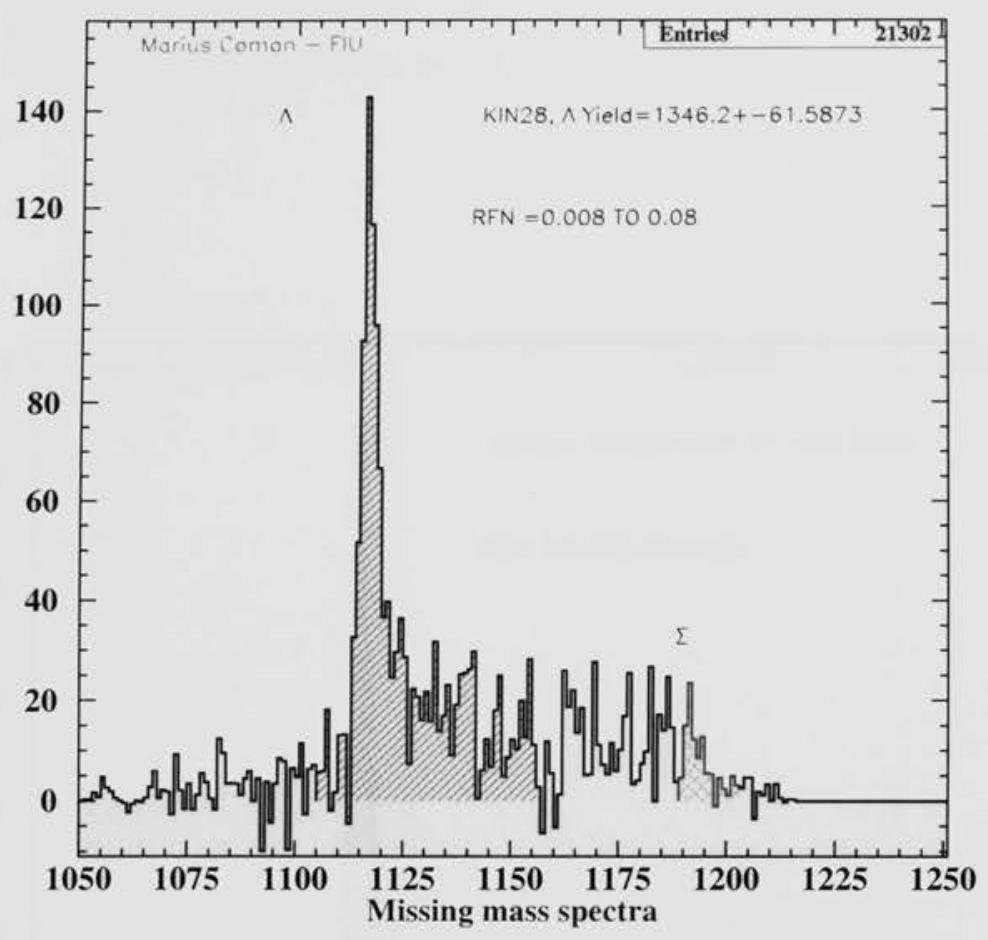

Figure 105: $\Lambda$ and $\Sigma$ Missing mass $\left(x\right.$ axis is in $\left.\mathrm{MeV} / \mathrm{c}^{2}\right)$, Kin. 28

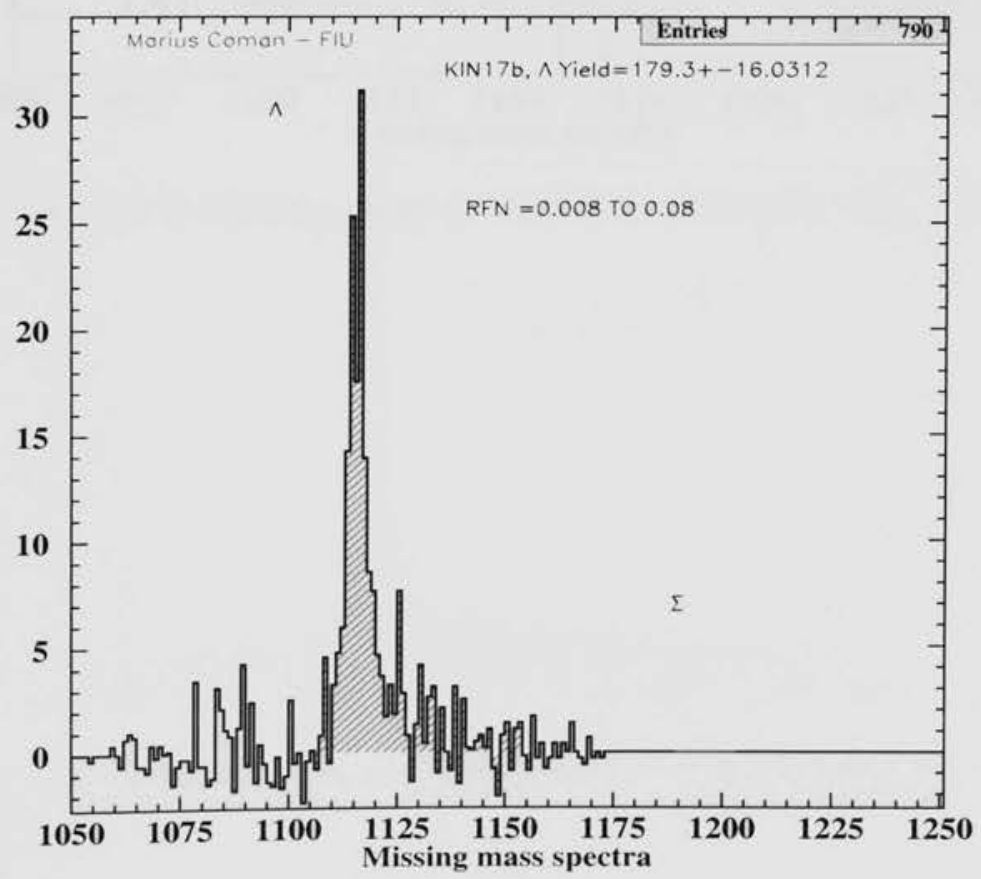

Figure 106: $\Lambda$ and $\Sigma$ Missing mass ( $x$ axis is in $\mathrm{MeV} / \mathrm{c}^{2}$ ), Kin. $17 \mathrm{~B}$ 


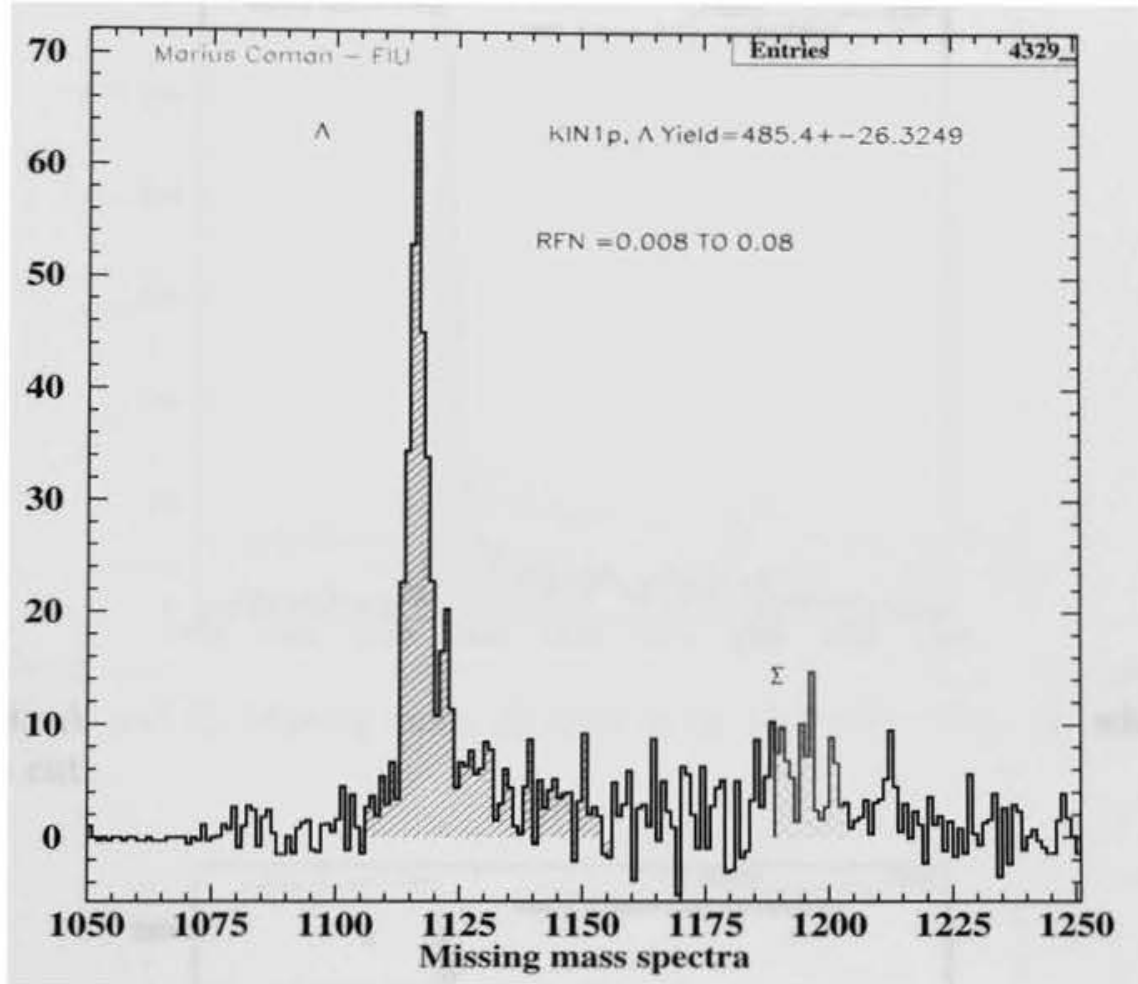

Figure 107: $\Lambda$ and $\Sigma$ Missing mass ( $x$ axis is in $\mathrm{MeV} / \mathrm{c}^{2}$ ), Kin. 1 Prime, 2002 
C E98-108 experiment: $\Lambda$ and $\Sigma^{0}$ missing mass spectra for some kinematics without the R-function cut applied (see Sect. 4.3)

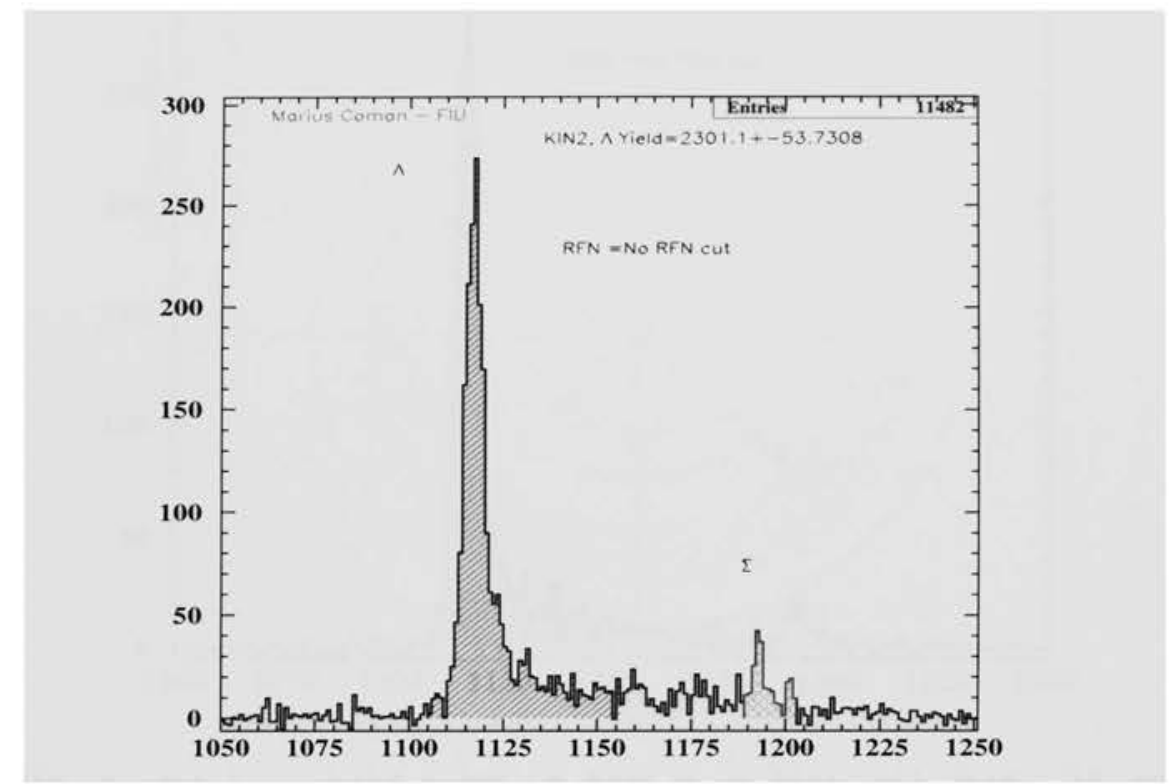

Figure 108: $\Lambda$ and $\Sigma$ Missing mass $\left(x\right.$ axis is in $\left.\mathrm{MeV} / \mathrm{c}^{2}\right)$, Kin. 2, without the R-function cut.

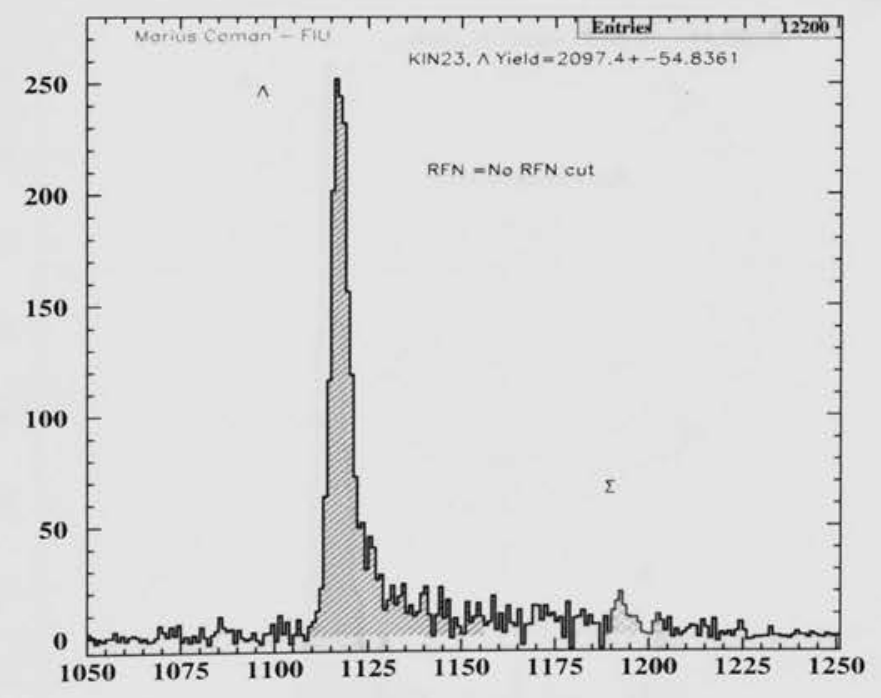

Figure 109: $\Lambda$ and $\Sigma$ Missing mass $\left(x\right.$ axis is in $\mathrm{MeV} / \mathrm{c}^{2}$ ), Kin. 23, without the R-function cut. 


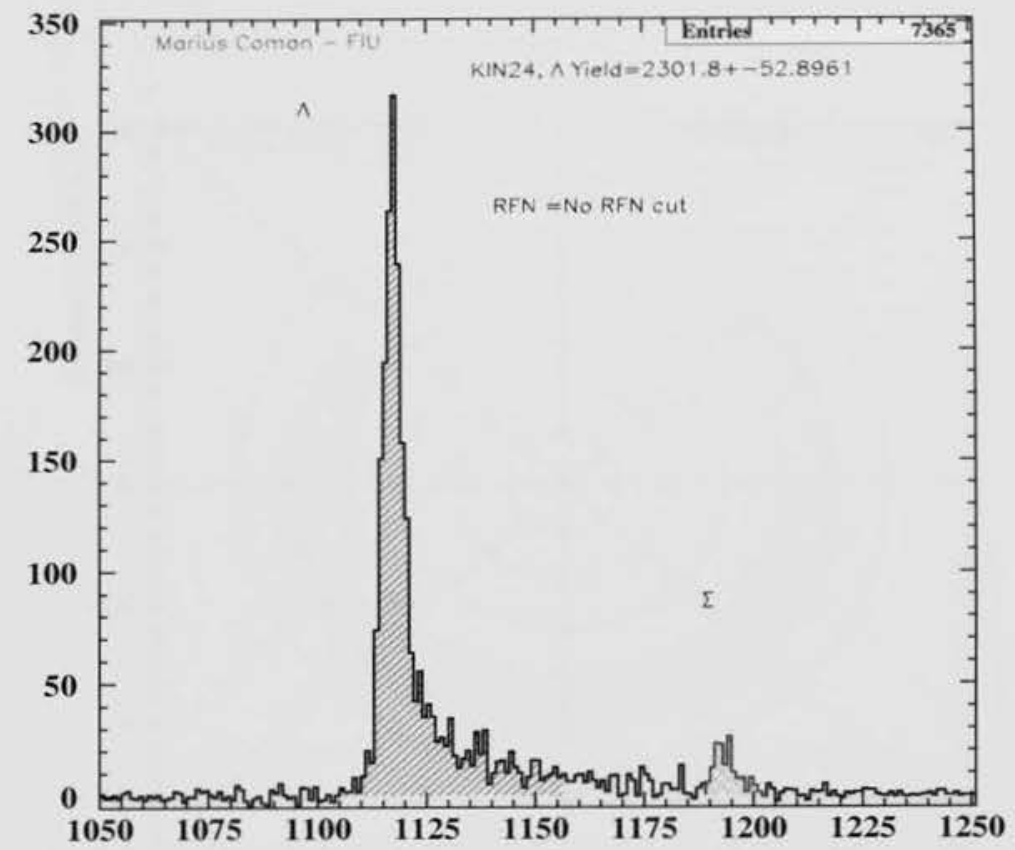

Figure 110: $\Lambda$ and $\Sigma$ Missing mass ( $x$ axis is in $\mathrm{MeV} / \mathrm{c}^{2}$ ), Kin. 24, without the R-function cut.

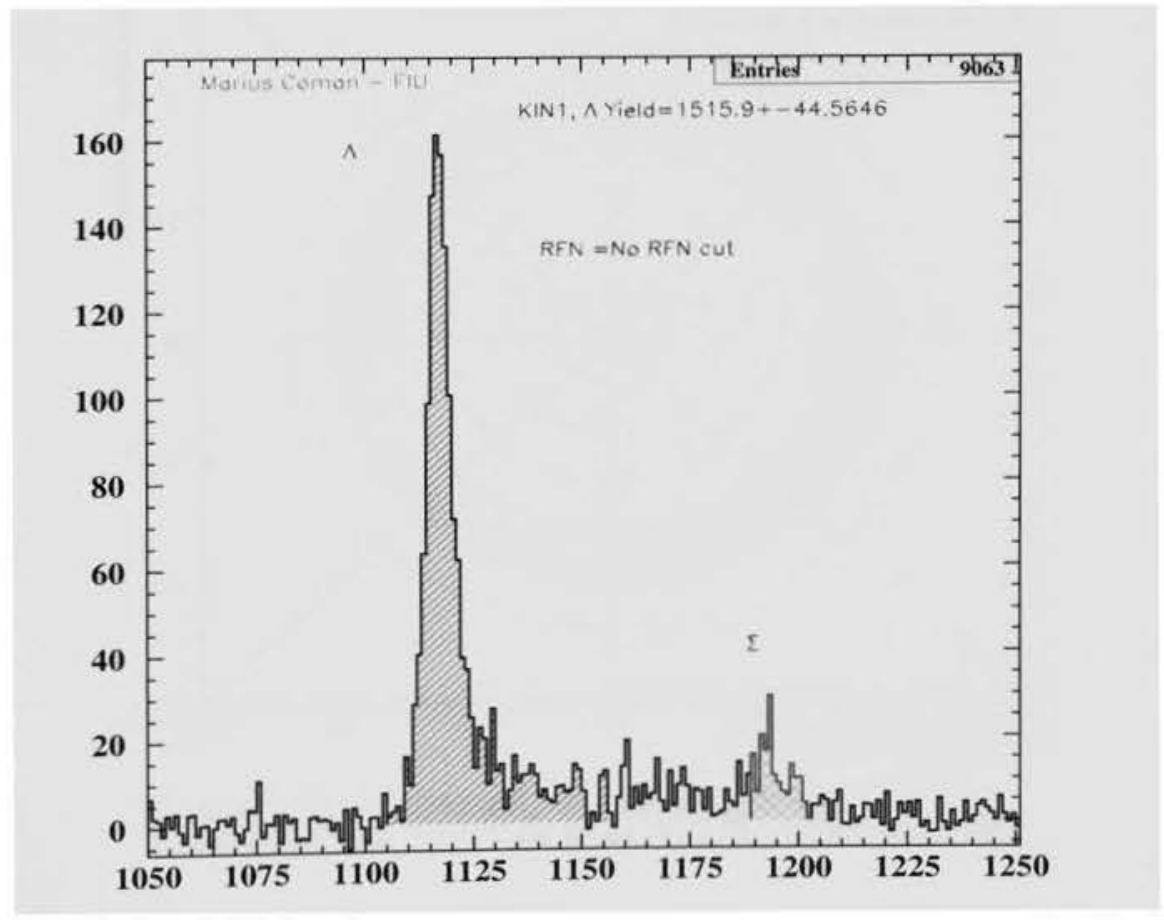

Figure 111: $\Lambda$ and $\Sigma$ Missing mass $\left(x\right.$ axis is in $\mathrm{MeV} / \mathrm{c}^{2}$ ), Kin. 1 , without the R-function cut. 
D E98-108 experiment: $\phi$ and $t$ coverage for all kinematics

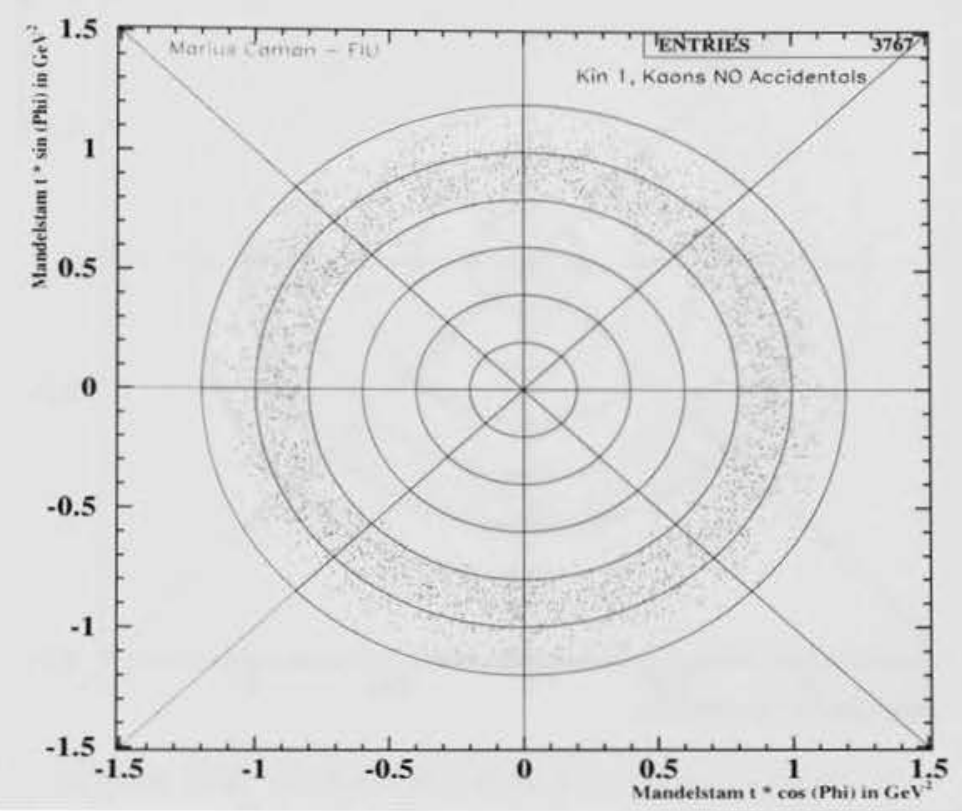

Figure 112: $\Lambda$ channel, $\phi$ and $t$ coverage, Kin 1 .

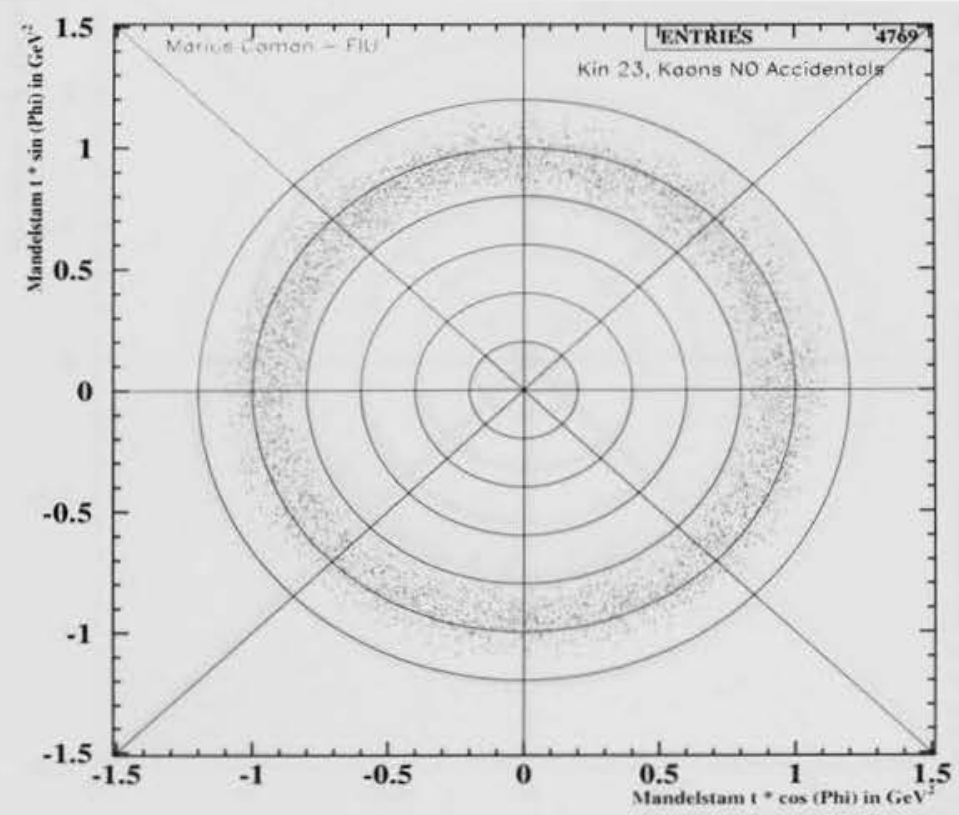

Figure 113: $\Lambda$ channel, $\phi$ and $t$ coverage, Kin 23. 


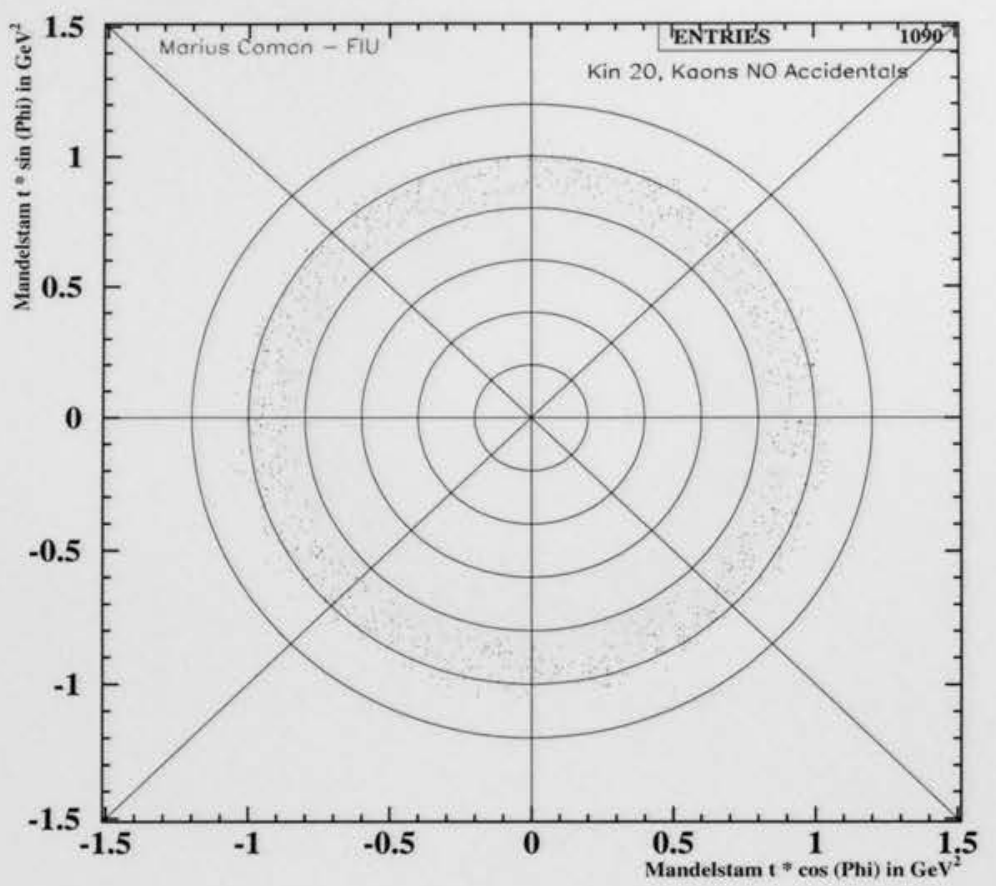

Figure 114: $\Lambda$ channel, $\phi$ and $t$ coverage, Kin 20 .

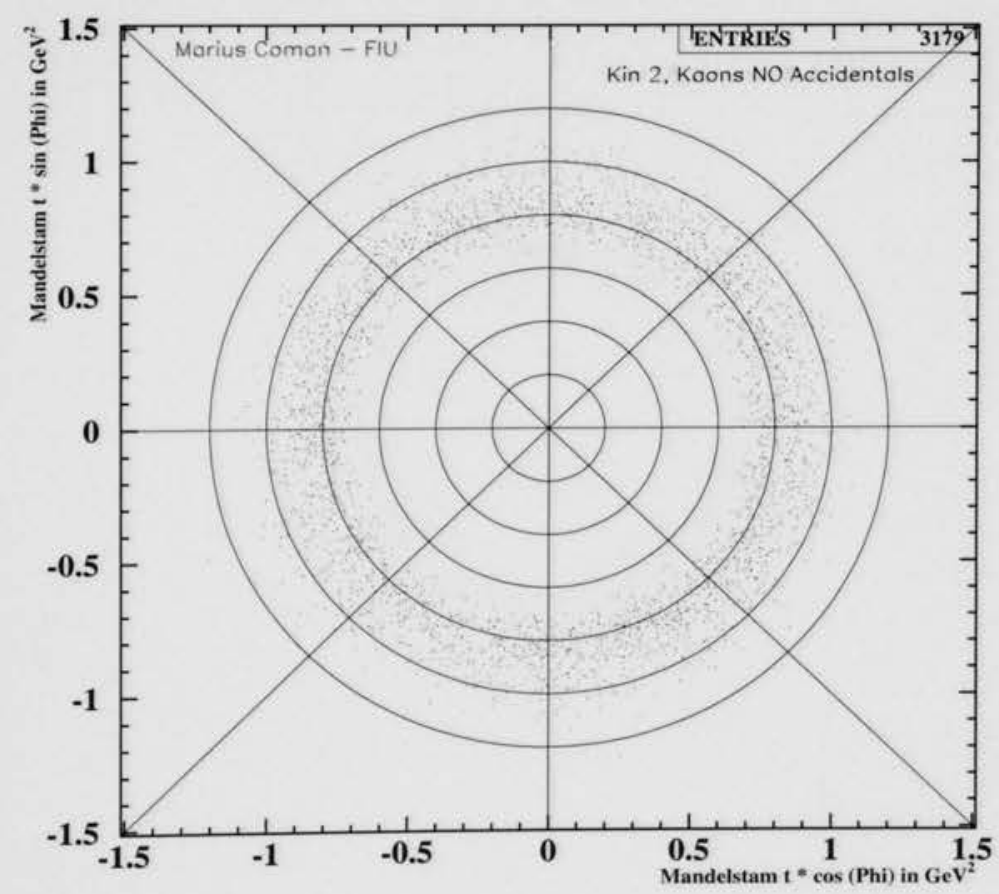

Figure 115: $\Lambda$ channel, $\phi$ and $t$ coverage, Kin 2. 


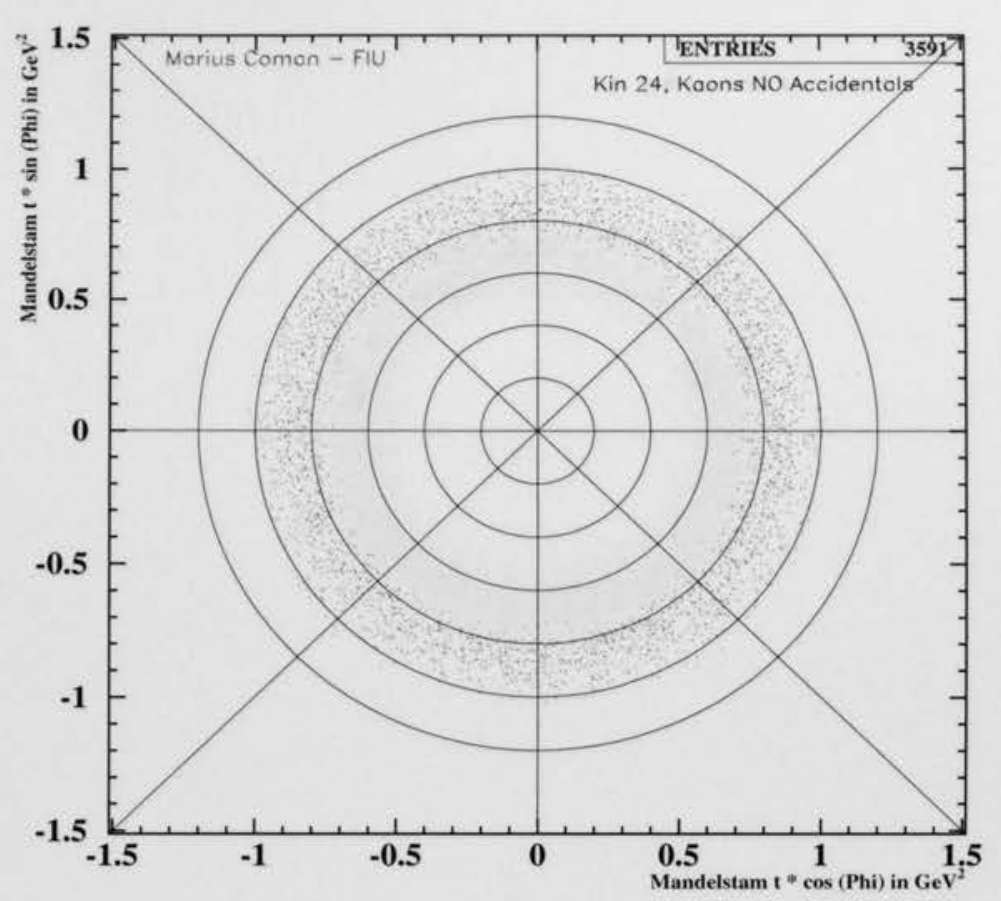

Figure 116: $\Lambda$ channel, $\phi$ and $t$ coverage, Kin 24 .

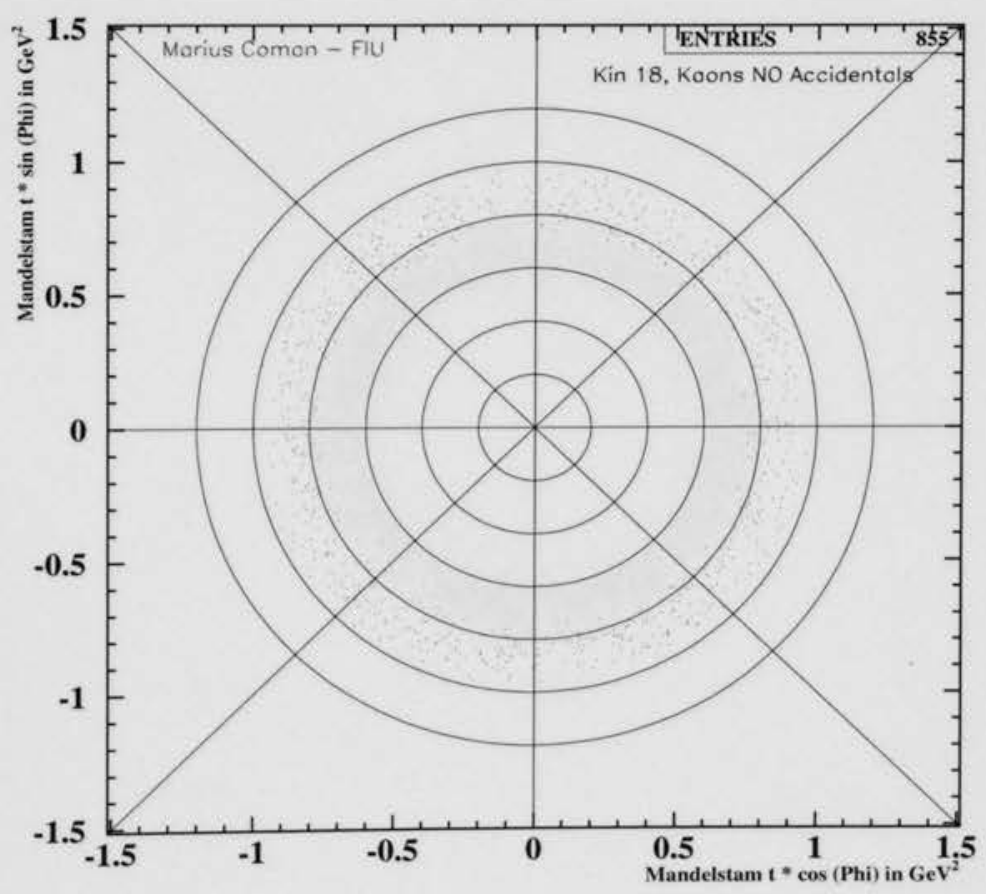

Figure 117: $\Lambda$ channel, $\phi$ and $t$ coverage, Kin 18. 


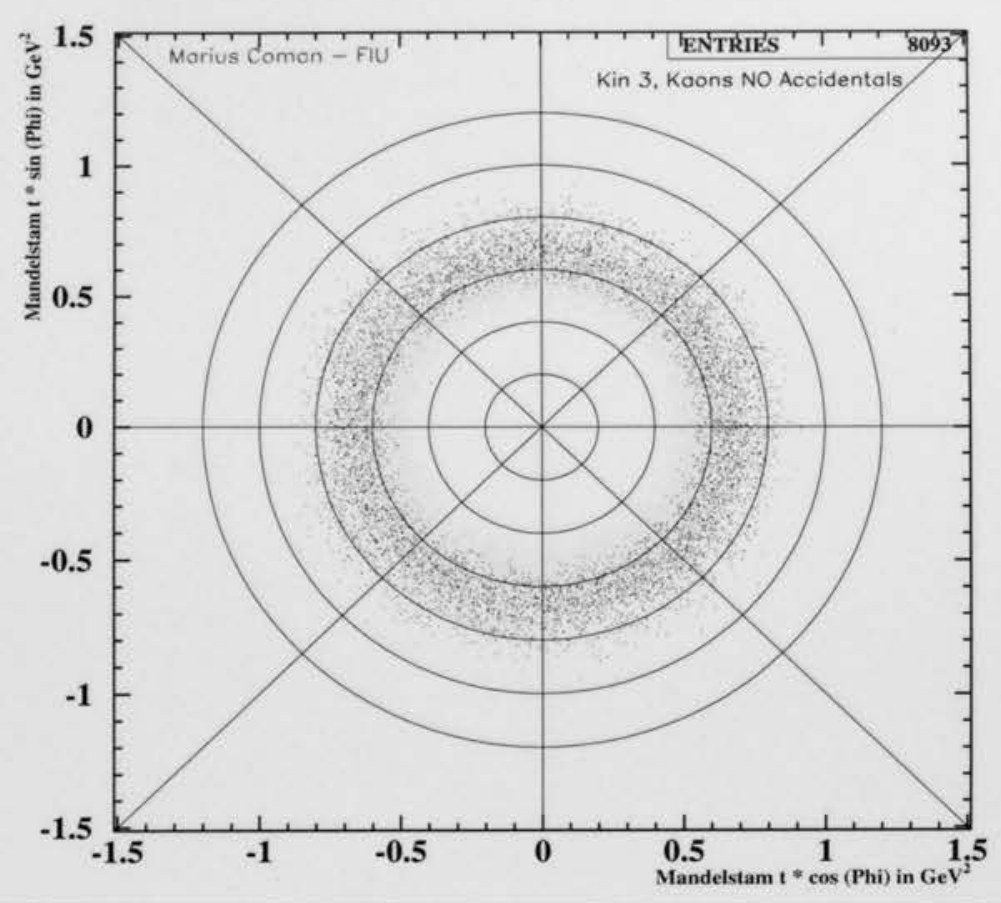

Figure 118: $\Lambda$ channel, $\phi$ and $t$ coverage, Kin 3 .

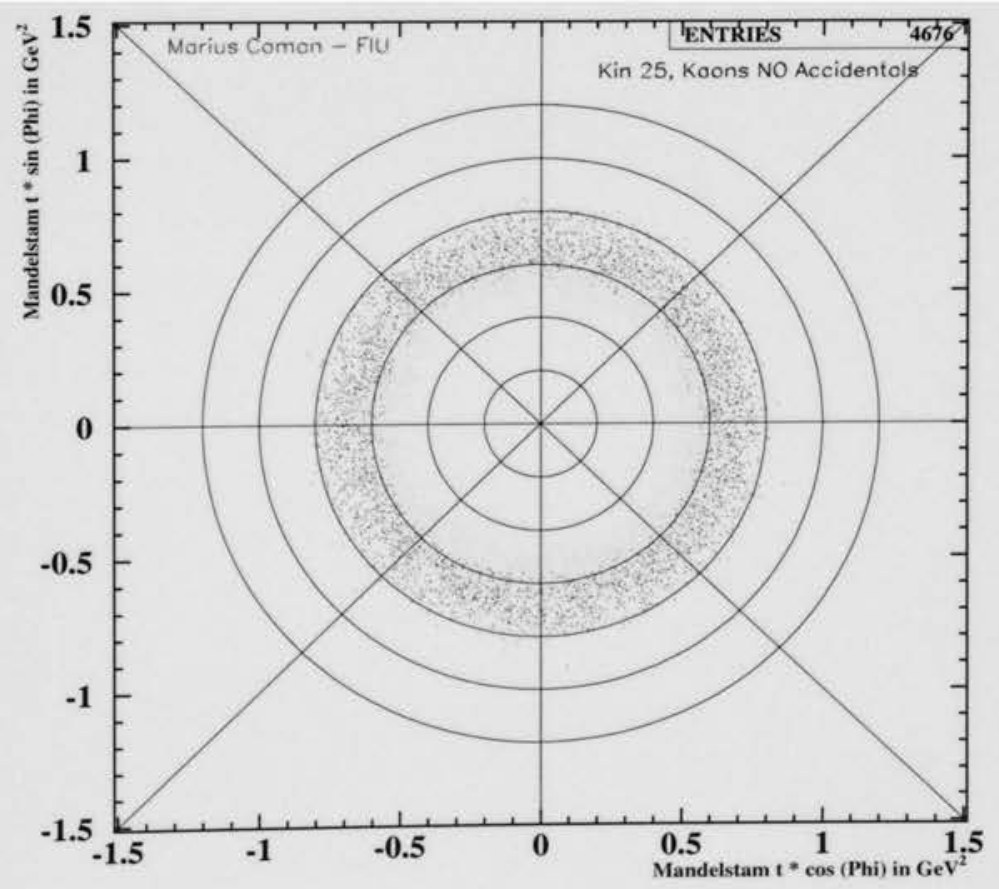

Figure 119: $\Lambda$ channel, $\phi$ and $t$ coverage, Kin 25 . 


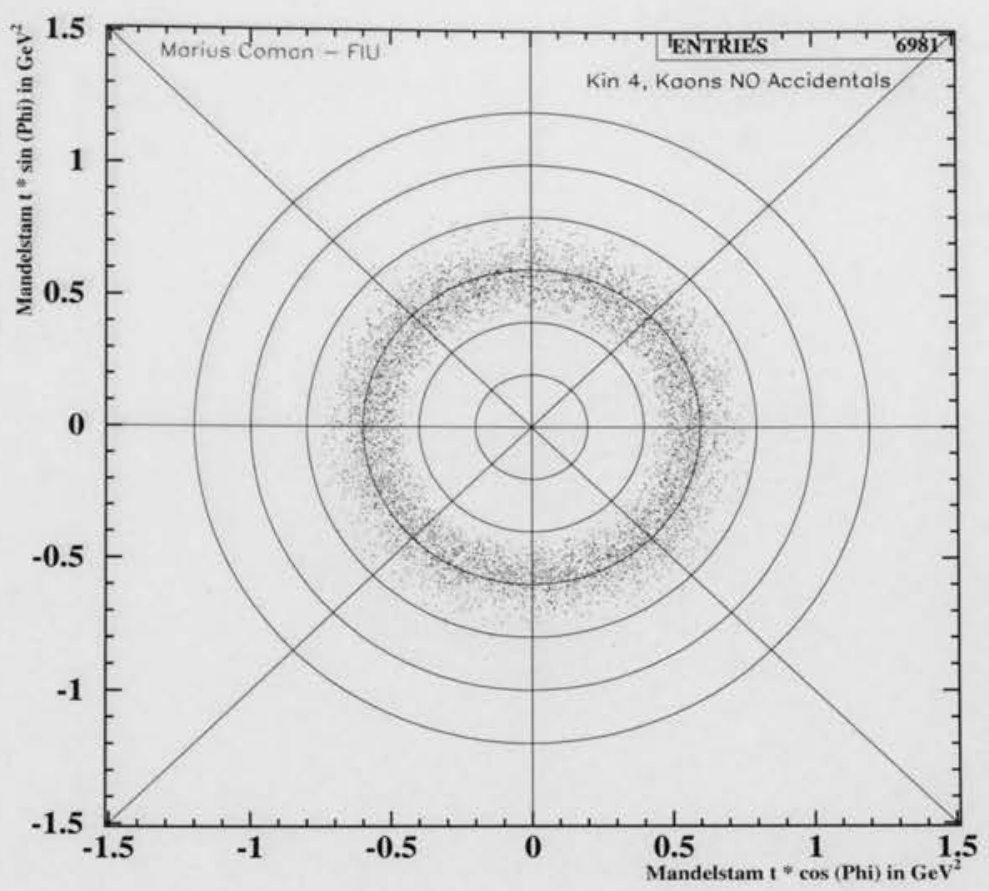

Figure 120: $\Lambda$ channel, $\phi$ and $t$ coverage, Kin 4 .

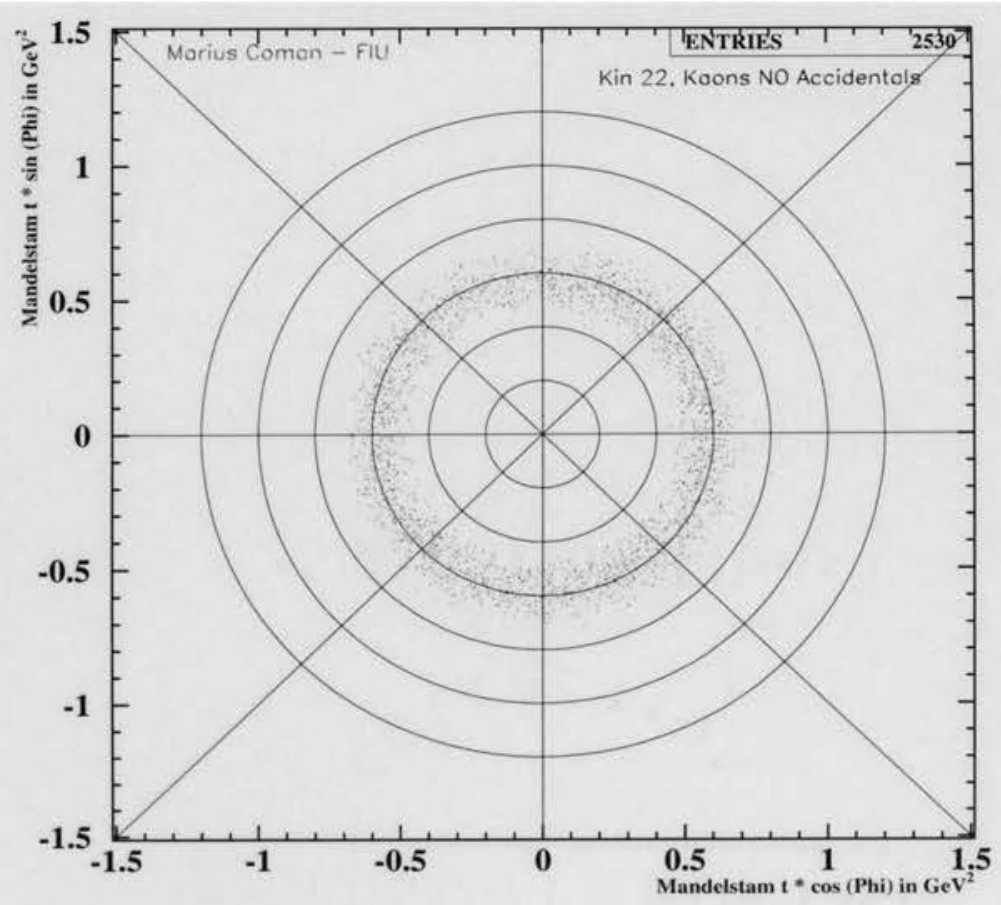

Figure 121: $\Lambda$ channel, $\phi$ and $t$ coverage, Kin 22 . 


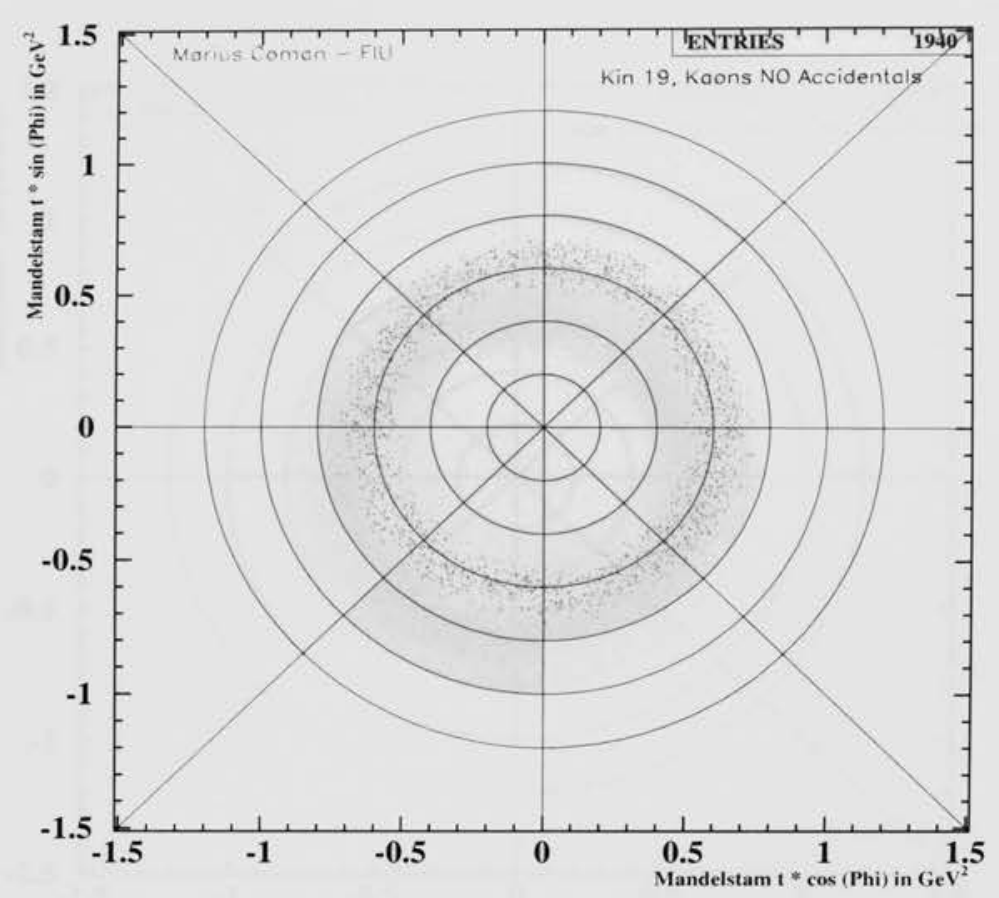

Figure 122: $\Lambda$ channel, $\phi$ and $t$ coverage, Kin 19.

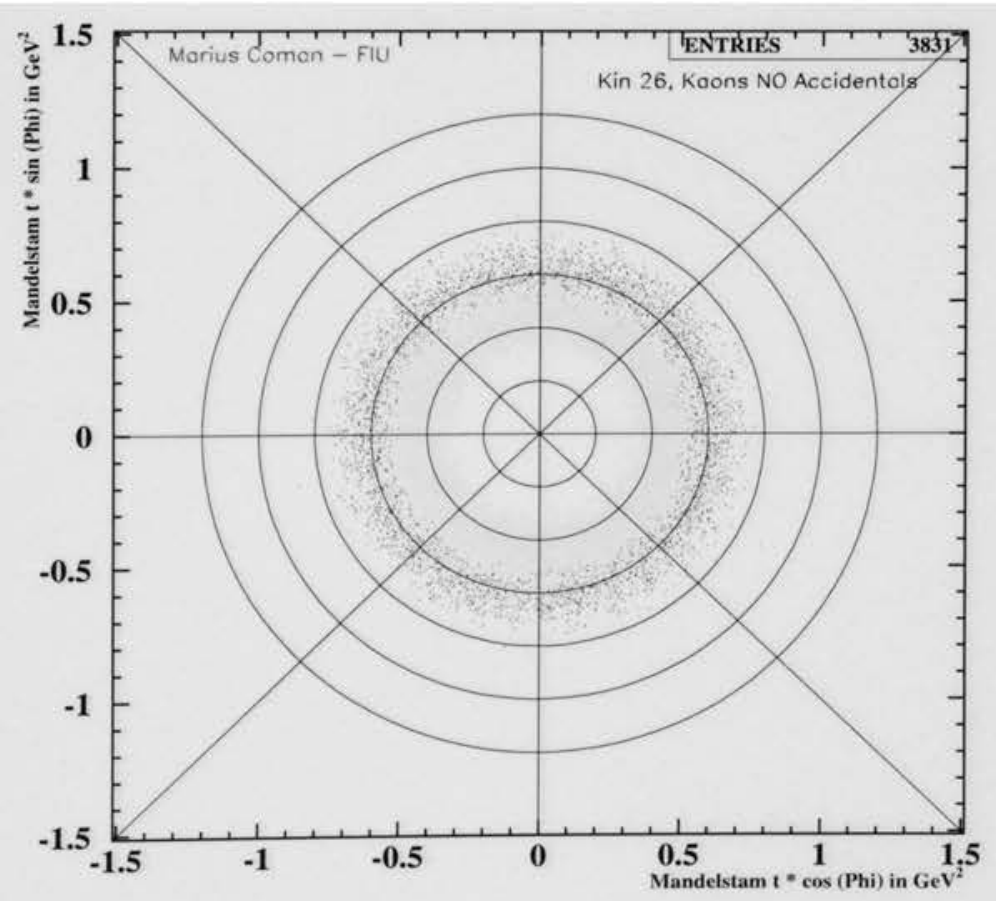

Figure 123: $\Lambda$ channel, $\phi$ and $t$ coverage, Kin 26. 


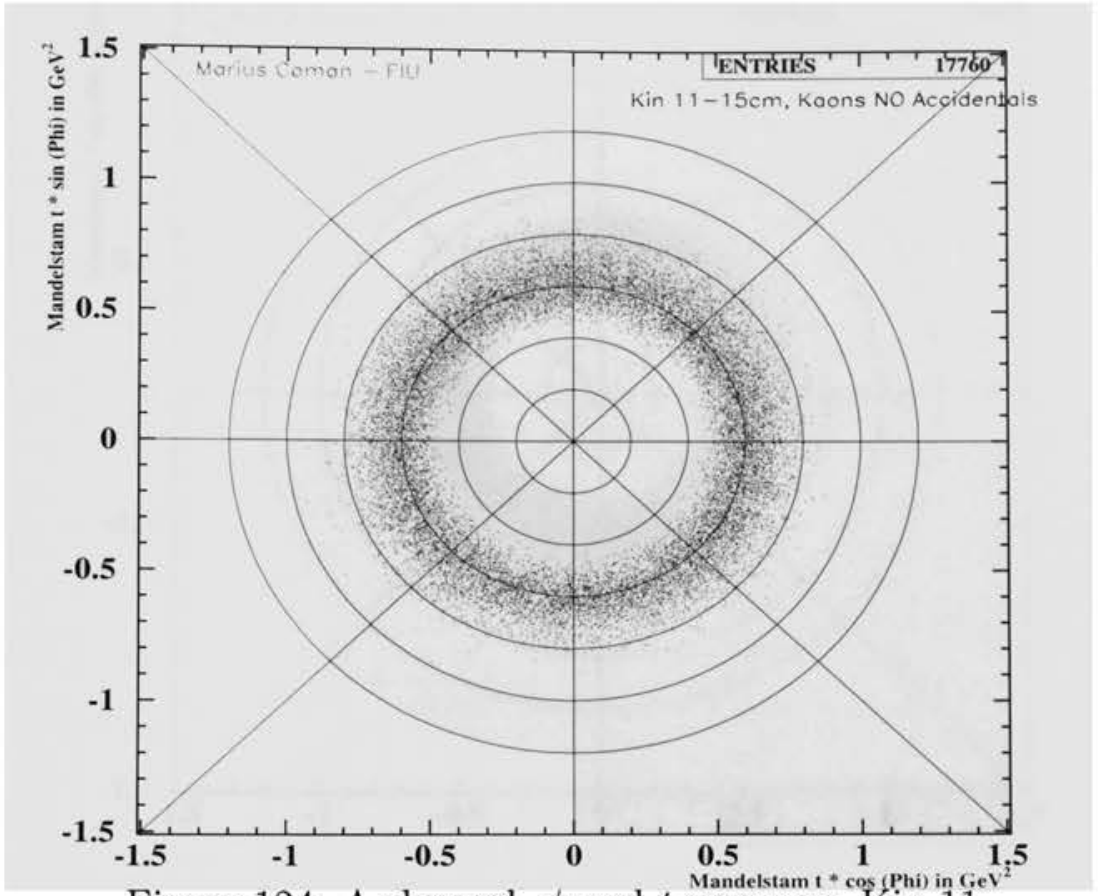

Figure 124: $\Lambda$ channel, $\phi$ and $t$ coverage, Kin 11 .

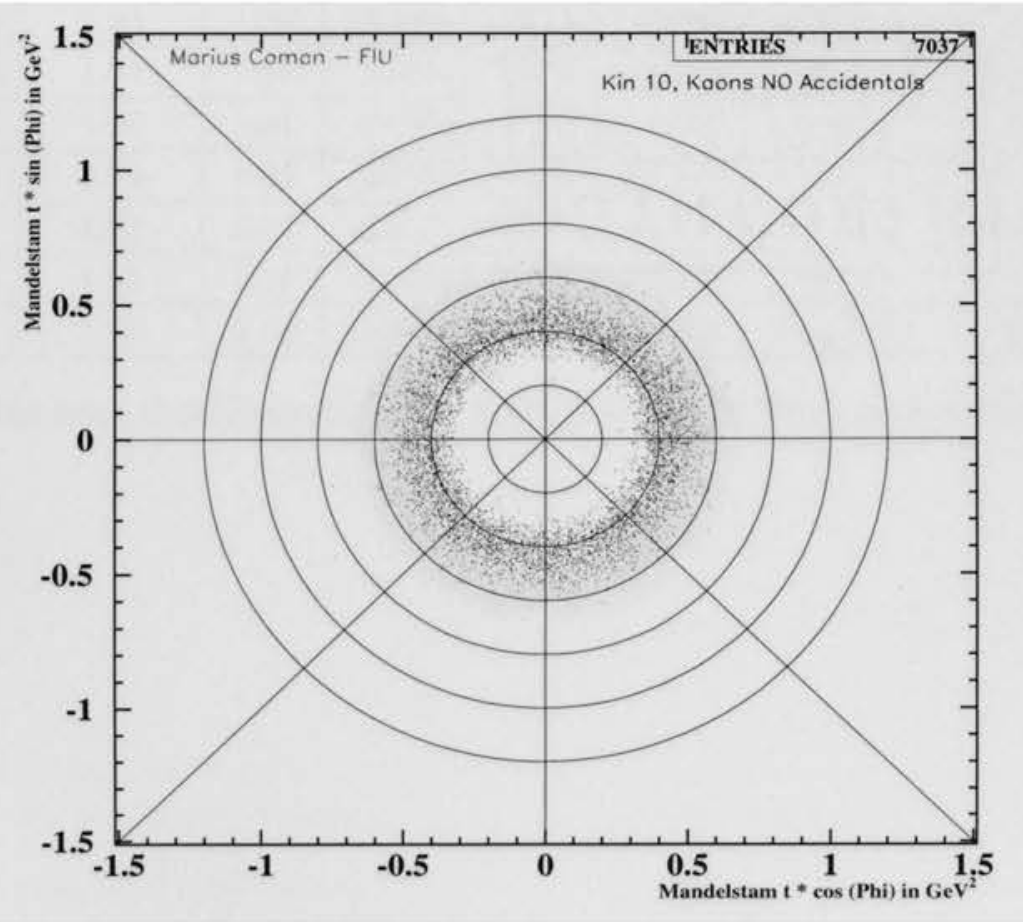

Figure 125: $\Lambda$ channel, $\phi$ and $t$ coverage, Kin 10. 


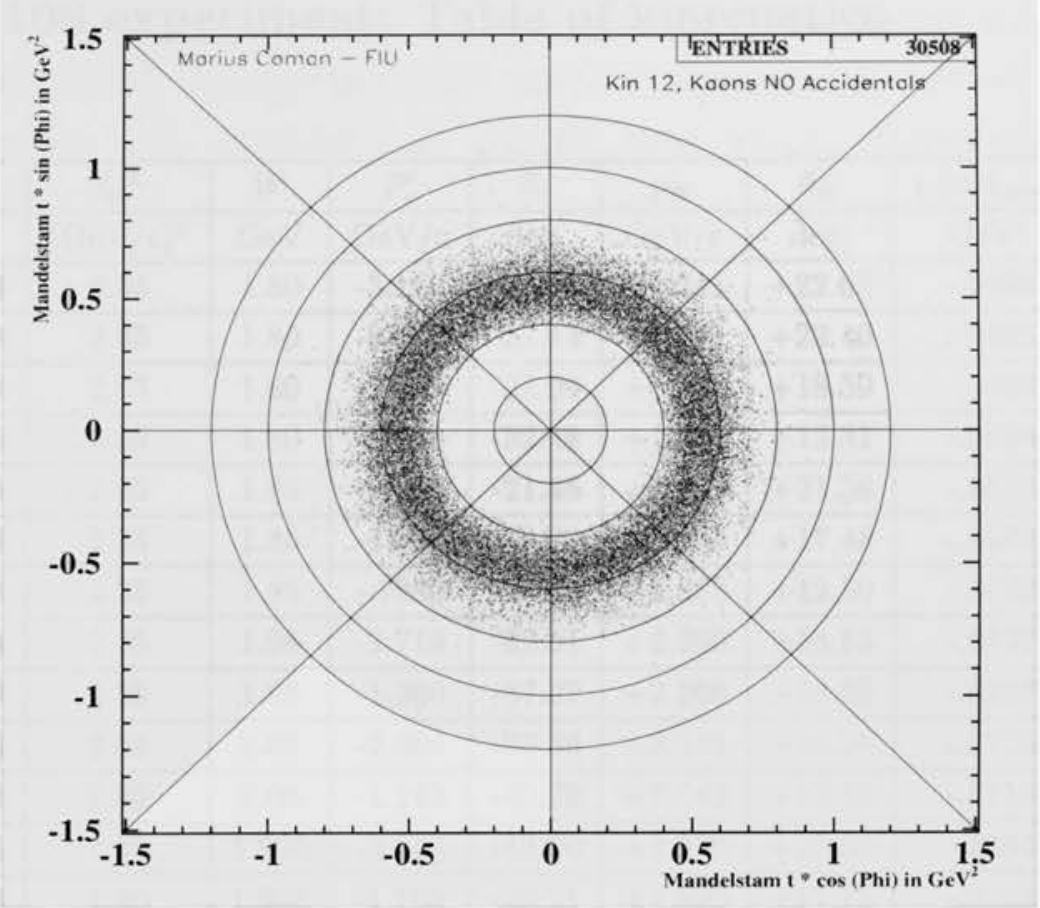

Figure 126: $\Lambda$ channel, $\phi$ and $t$ coverage, Kin 11.

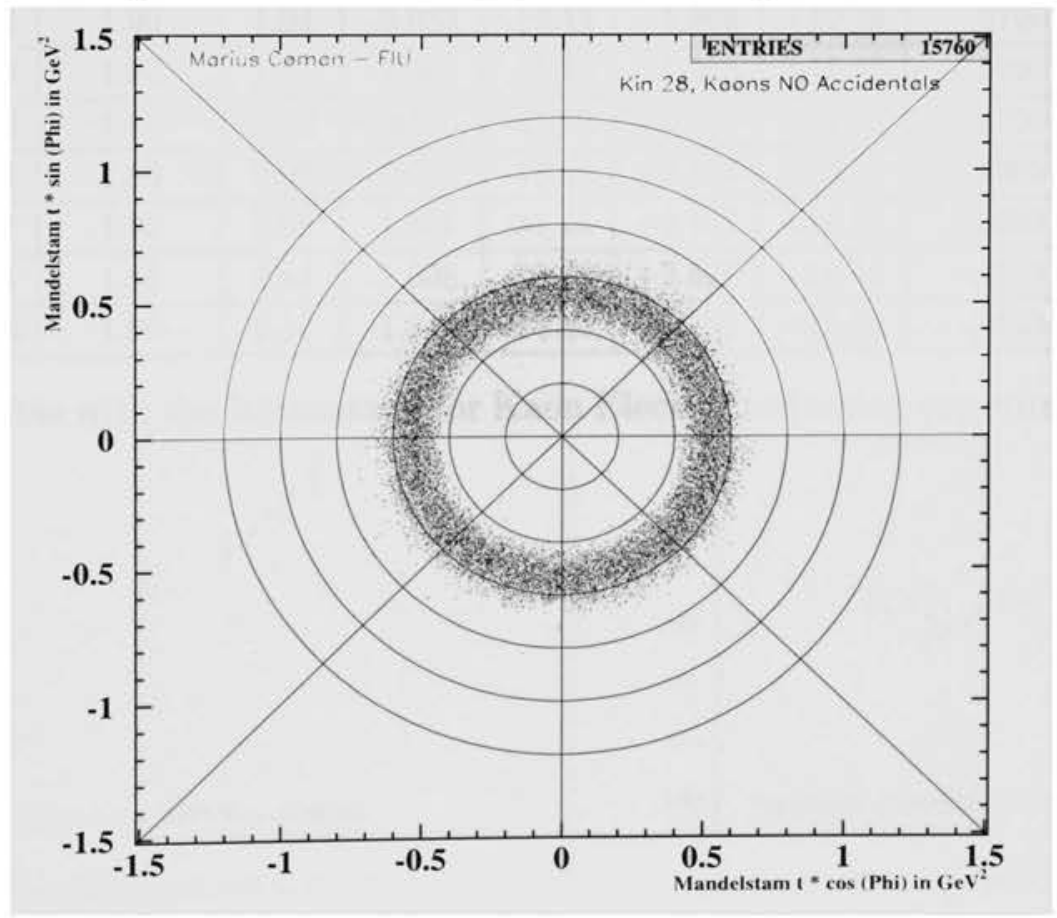

Figure 127: $\Lambda$ channel, $\phi$ and $t$ coverage, Kin 10. 
E E98-108 experiment: Table of kinematics

\begin{tabular}{|c|c|c|c|c|c|c|c|c|c|}
\hline Kin & $E_{0}$ & $Q^{2}$ & $W$ & $P_{e}^{\prime}$ & $\theta_{e}$ & $p_{K}$ & $\theta_{K}$ & $\mathrm{t}\left(=t_{\min }\right)$ & $\epsilon$ \\
\hline$\#$ & $\mathrm{GeV}$ & $(\mathrm{GeV} / \mathrm{c})^{2}$ & $\mathrm{GeV}$ & $\mathrm{GeV} / \mathrm{c}$ & $\mathrm{deg}$. & $\mathrm{GeV} / \mathrm{c}$ & $\mathrm{deg}$. & $\mathrm{GeV}^{2}$ & \\
\hline $1^{\prime}$ & 5.7544 & 2.35 & 1.80 & -3.180 & -20.43 & +1.741 & +22.66 & -.9498 & .8071 \\
\hline 1 & 5.6144 & 2.35 & 1.80 & -3.077 & -21.14 & +1.741 & +22.40 & -.9498 & .7960 \\
\hline 23 & 4.2383 & 2.35 & 1.80 & -1.688 & -32.99 & +1.741 & +18.59 & -.9498 & .6078 \\
\hline 20 & 3.4006 & 2.35 & 1.80 & -.8853 & -52.48 & +1.741 & +13.81 & -.9498 & .3586 \\
\hline 2 & 5.6144 & 2.35 & 1.85 & -2.981 & -21.48 & +1.893 & +21.38 & -.8562 & .7812 \\
\hline 24 & 4.2383 & 2.35 & 1.85 & -1.593 & -33.99 & +1.893 & +17.48 & -.8562 & .5790 \\
\hline 18 & 3.4006 & 2.35 & 1.85 & -.7880 & -55.89 & +1.893 & +12.50 & -.8562 & .3134 \\
\hline 3 & 5.6144 & 2.35 & 1.98 & -2.719 & -22.51 & +2.266 & +18.83 & -.6737 & .7368 \\
\hline 25 & 4.2383 & 2.35 & 1.98 & -1.360 & -37.27 & +2.266 & +14.65 & -.6737 & .4936 \\
\hline 4 & 5.6144 & 2.35 & 2.08 & -2.504 & -23.46 & +2.542 & +16.98 & -.5716 & .6962 \\
\hline 22 & 4.2383 & 2.35 & 2.08 & -1.143 & -40.79 & +2.542 & +12.51 & -.5716 & .4169 \\
\hline 11 & 5.7544 & 1.90 & 1.905 & -3.145 & -18.60 & +1.970 & +20.69 & -.5994 & .8107 \\
\hline 26 & 4.2383 & 1.90 & 1.905 & -1.765 & -29.21 & +1.890 & +17.75 & -.6183 & .6366 \\
\hline 19 & 3.4006 & 1.90 & 1.905 & -.9279 & -45.70 & +1.890 & +13.59 & -.6183 & .4012 \\
\hline 5 & 5.6144 & 1.90 & 1.94 & -3.039 & -19.11 & +1.986 & +20.29 & -.5790 & .7997 \\
\hline 27 & 4.2383 & 1.90 & 1.94 & -1.683 & -29.93 & +1.986 & +16.85 & -.5790 & .6129 \\
\hline 17 & 3.4006 & 1.90 & 1.94 & -.8460 & -47.99 & +1.986 & +12.53 & -.5790 & .3635 \\
\hline 12 & 5.7544 & 1.90 & 2.00 & -3.018 & -18.84 & +2.147 & +19.30 & -.5203 & .7921 \\
\hline 28 & 4.2383 & 1.90 & 2.00 & -1.557 & -31.15 & +2.147 & +15.52 & -.5203 & .5746 \\
\hline 10 & 5.6144 & 1.90 & 2.14 & -2.608 & -20.64 & +2.521 & +16.41 & -.4143 & .7262 \\
\hline 21 & 4.2383 & 1.90 & 2.14 & -1.248 & -34.90 & +2.521 & +12.55 & -.4143 & .4709 \\
\hline
\end{tabular}

Table 15: Table with the Kinematics for Kaon Electroproduction experiment E98-108 at JLAB. 
F E98-108 experiment: Rosenbluth separation; figures with separated longitudinal and transverse cross sections; Inner errors are statistical outer are total (systematic and statistical)

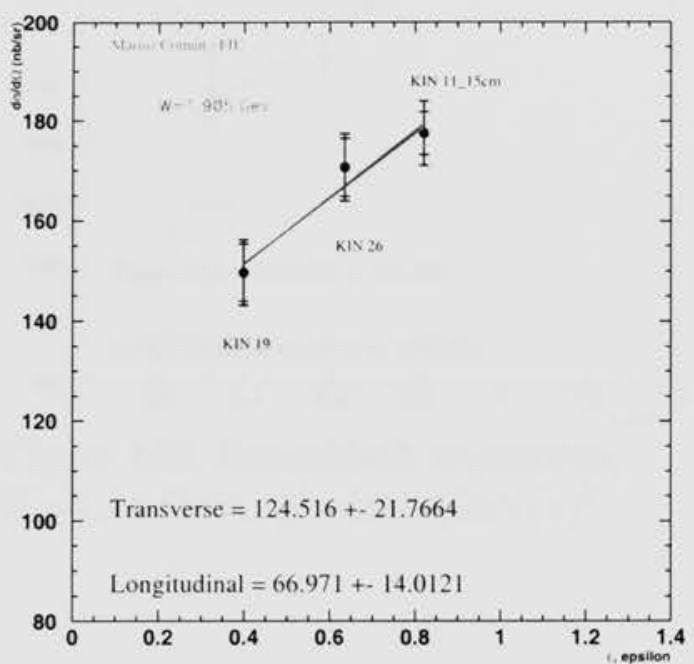

Figure 128: Rosenbluth separation, $W=1.905 \mathrm{GeV}, Q^{2}=1.90(\mathrm{GeV} / \mathrm{c})^{2}$.

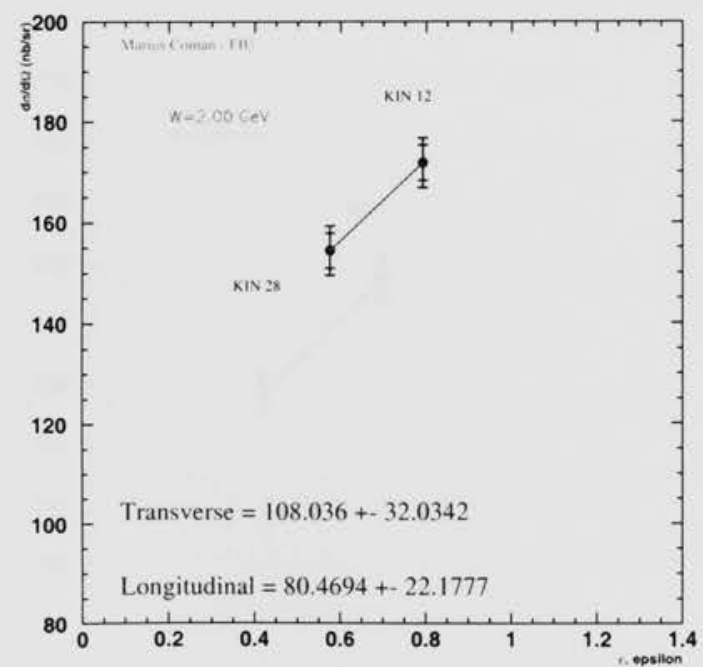

Figure 130: Rosenbluth separation, $W=2 . \mathrm{GeV}, Q^{2}=1.90(\mathrm{GeV} / \mathrm{c})^{2}$.

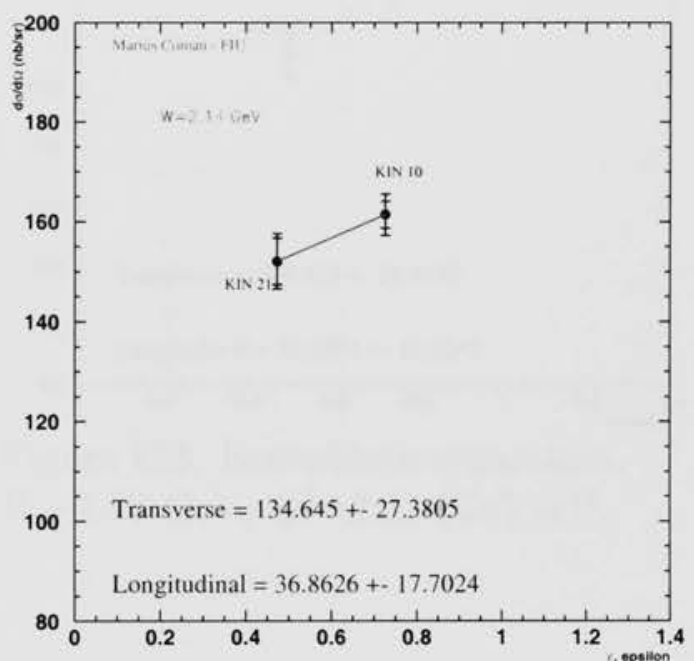

Figure 129: Rosenbluth separation, $W=2.14 \mathrm{GeV}, Q^{2}=1.90(\mathrm{GeV} / \mathrm{c})^{2}$.

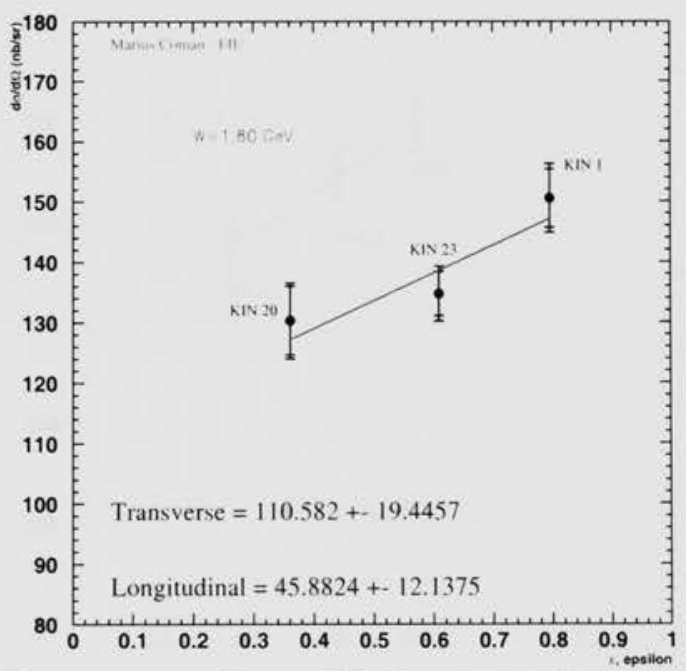

Figure 131: Rosenbluth separation, $W=1.8 \mathrm{GeV}, Q^{2}=2.35(\mathrm{GeV} / \mathrm{c})^{2}$. 


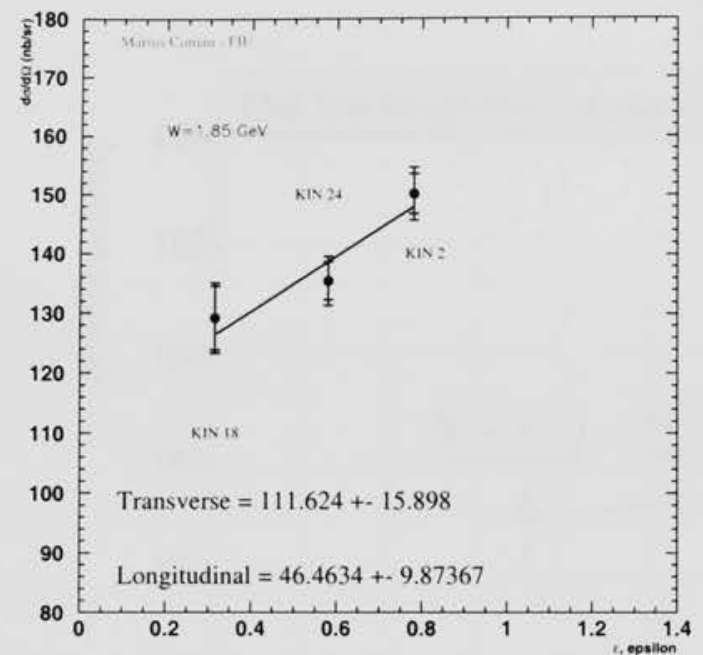

Figure 132: Rosenbluth separation, $W=1.85 \mathrm{GeV}, Q^{2}=2.35(\mathrm{GeV} / \mathrm{c})^{2}$.

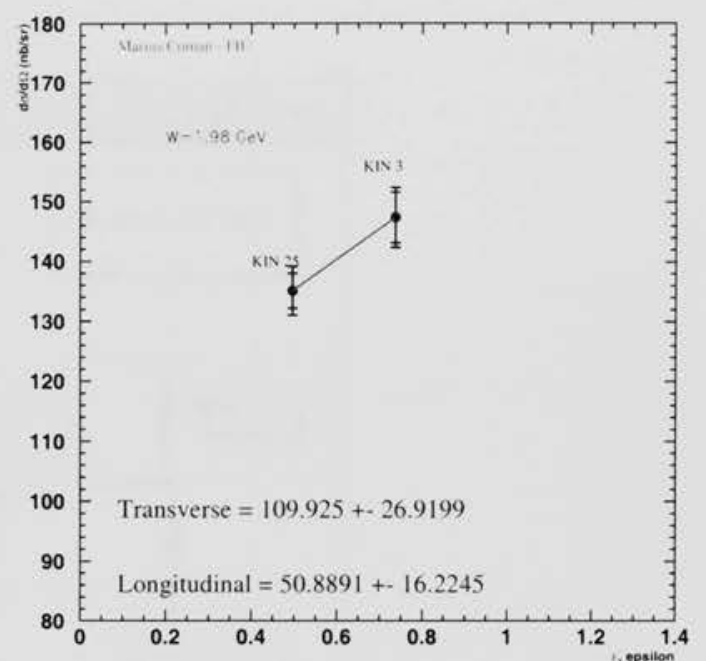

Figure 133: Rosenbluth separation, $W=1.98 \mathrm{GeV}, Q^{2}=2.35(\mathrm{GeV} / \mathrm{c})^{2}$.

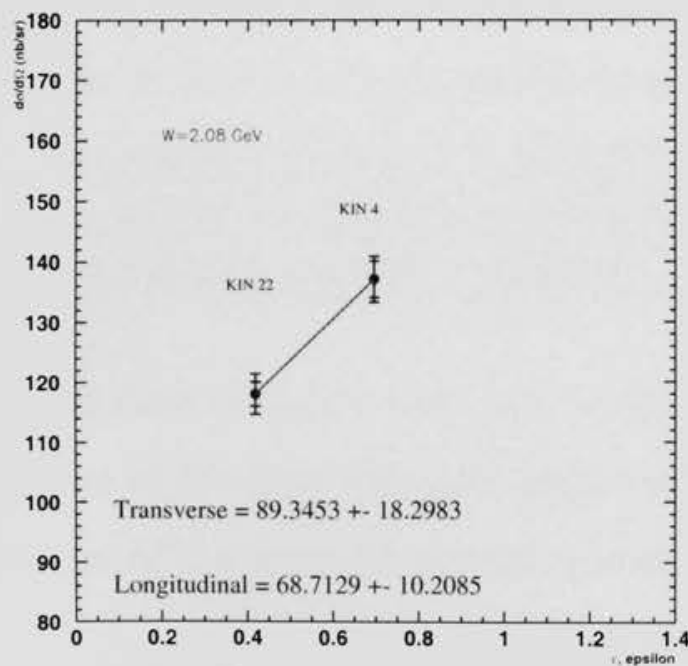

Figure 134: Rosenbluth separation, $W=2.08 \mathrm{GeV}, Q^{2}=2.35(\mathrm{GeV} / \mathrm{c})^{2}$.

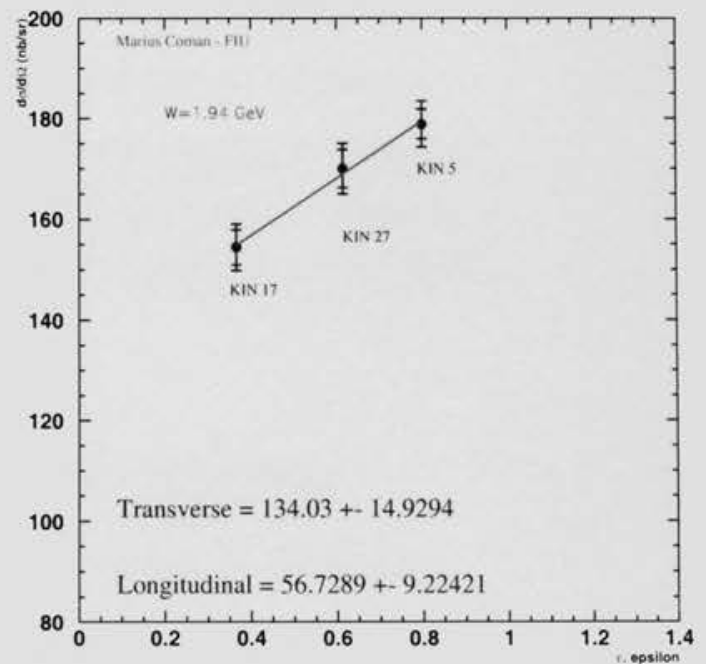

Figure 135: Rosenbluth separation, $W=1.94 \mathrm{GeV}, Q^{2}=1.90(\mathrm{GeV} / \mathrm{c})^{2}$. 


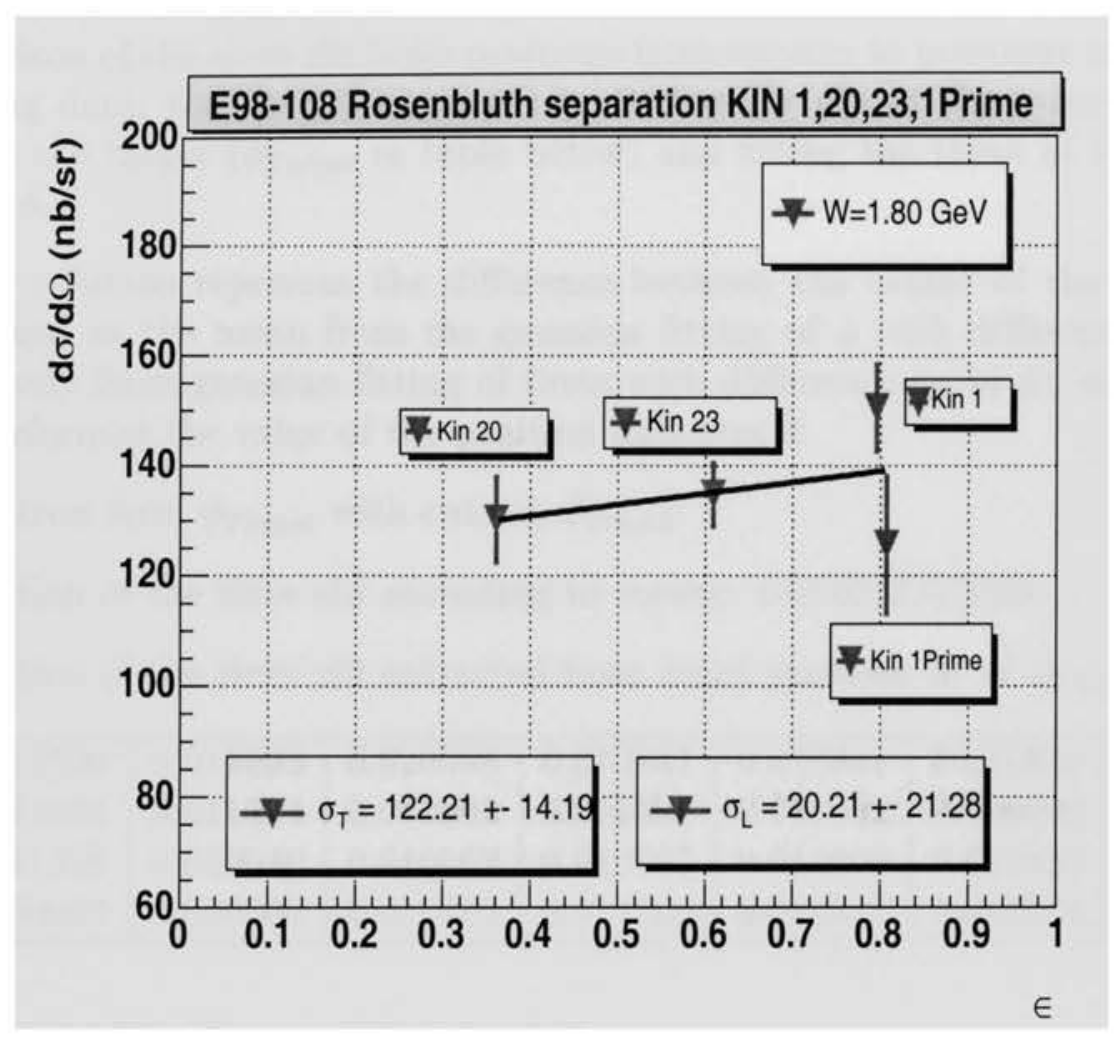

Figure 136: Rosenbluth separation, $W=1.80 \mathrm{GeV}, Q^{2}=2.35(\mathrm{GeV} / \mathrm{c})^{2}$. Kin 1 prime included. 


\section{G Sieve slit position determination; comparison of position as extracted from survey and as determined from data}

Comparison of the sieve slit holes positions from surveys to positions as resulted from analysing data; the data is extracted by fitting the phi at the target with cuts on theta at the target ( $\phi_{\text {Target }}$ in table below) and fitting the theta at the target with cuts on $\phi$.

The columns represent the difference between the center of the holes (centers determined as the mean from the gaussian fitting of $\phi$ with different cuts in theta respectively from gaussian fitting of theta with different cuts in $\phi$ ); where no fit has been performed the value of the position indicates 0 .

Electron Arm $\phi_{\text {Target }}$ with cuts on $\theta_{\text {Target }}$ :

Position of the sieve slit according to survey: 0.010633772863 ;

Position of the sieve slit extracted from multi gaussian fit of $\phi_{\text {Target }}$ :

\begin{tabular}{|l|l|l|l|l|l|l|}
\hline 0.011596 & 0.011093 & 0.010588 & 0.010841 & 0.010841 & 0.010841 & 0.010336 \\
0.011093 & 0.011344 & 0.011093 & 0.011092 & 0.011092 & 0.000000 & 0.000000 \\
0.011328 & 0.010940 & 0.010562 & 0.010727 & 0.011006 & 0.010853 & 0.010206 \\
0.000000 & 0.000000 & 0.010822 & 0.011106 & 0.010887 & 0.011078 & 0.010687 \\
\hline
\end{tabular}

Electron Arm $\theta_{\text {Target }}$ with cuts on $\phi_{\text {Target }}$ :

Position of the sieve slit according to survey: 0.021267545725;

Position of the sieve slit extracted from multi gaussian fit of $\theta_{\text {Target }}$

\begin{tabular}{|c|c|c|c|c|c|}
\hline 0.020504 & 0.019496 & 0.020168 & 0.019832 & 0.020840 & 0.00000 \\
0.019826 & 0.019868 & 0.019903 & 0.019997 & 0.020734 & 0.02079 \\
0.000000 & 0.017200 & 0.000000 & 0.021366 & 0.000000 & 0.021599 \\
\hline
\end{tabular}

Hadron Arm $\phi_{\text {TARGET }}$ with cuts on $\theta_{\text {Target }}$ :

Position of the sieve slit according to survey: 0.010554758085;

Position of the sieve slit extracted from multi gaussian fit of $\phi_{\text {Target }}$

\begin{tabular}{|l|l|l|l|l|}
\hline 0.010588 & 0.009832 & 0.010588 & 0.009832 & 0.009328 \\
0.010084 & 0.010588 & 0.010589 & 0.009831 & 0.010336 \\
0.010376 & 0.009915 & 0.010246 & 0.009867 & 0.009411 \\
0.010125 & 0.010673 & 0.010410 & 0.010113 & 0.010445 \\
\hline
\end{tabular}


Hadron Arm $\theta_{\text {Target }}$ with cuts on $\phi_{T A R G E T}$ :

Position of the sieve slit according to survey: 0.02110951617;

Position of the sieve slit extracted from multi gaussian fit of $\theta_{\text {Target }}$

\begin{tabular}{|c|c|c|c|c|c|}
\hline 0.019826 & 0.019868 & 0.019903 & 0.019997 & 0.020734 & 0.02079 \\
0.027212 & 0.020529 & 0.020568 & 0.020939 & 0.020502 & 0.020349 \\
0.000000 & 0.021186 & 0.021332 & 0.021433 & 0.021386 & 0.021898 \\
\hline
\end{tabular}


H E98-108 experiment: Phase space coverage for $\Lambda$ and $\Sigma^{0}$

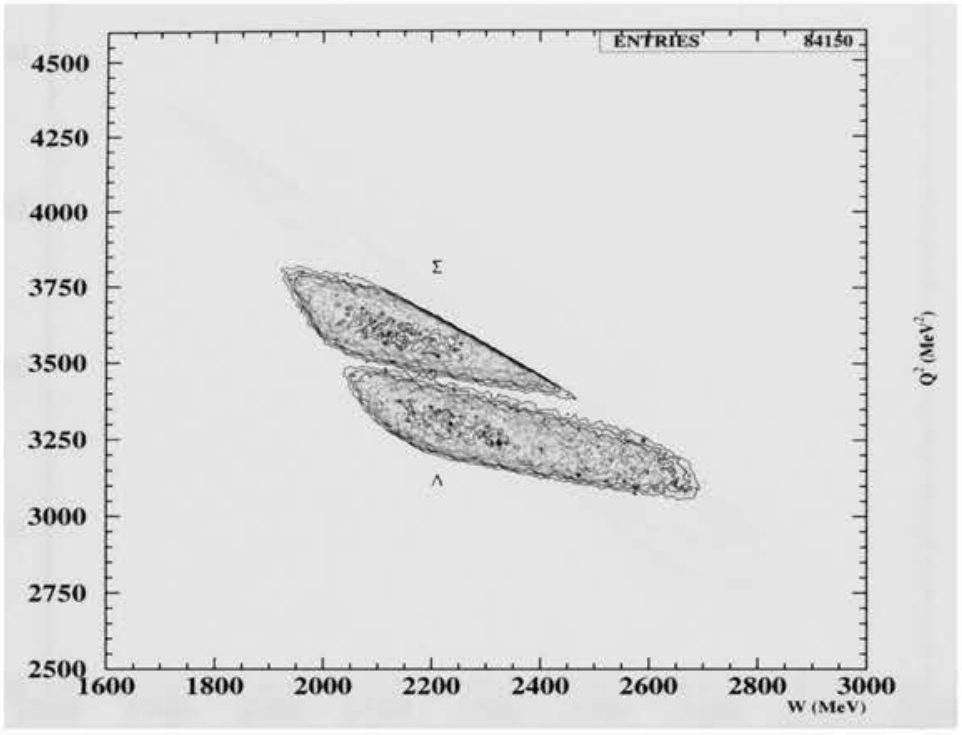

Figure 137: $W$ versus $Q^{2}$ phase space (for both $\Lambda$ and $\Sigma$ channels) for Kin 1 .

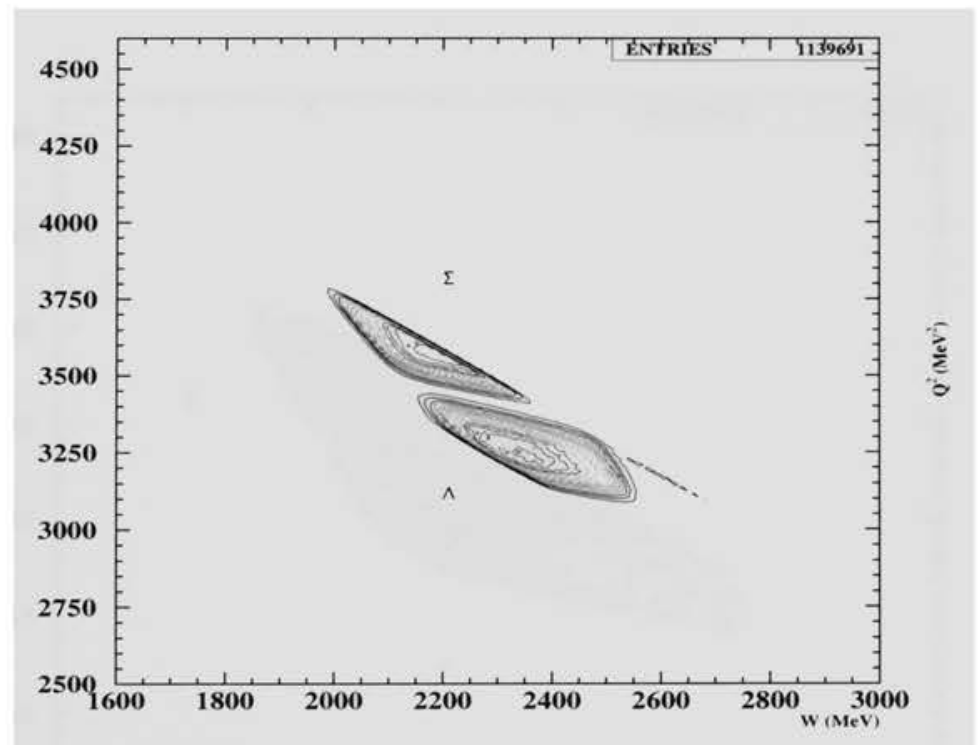

Figure 138: $W$ versus $Q^{2}$ phase space (for both $\Lambda$ and $\Sigma$ channels) for Kin 23 . 


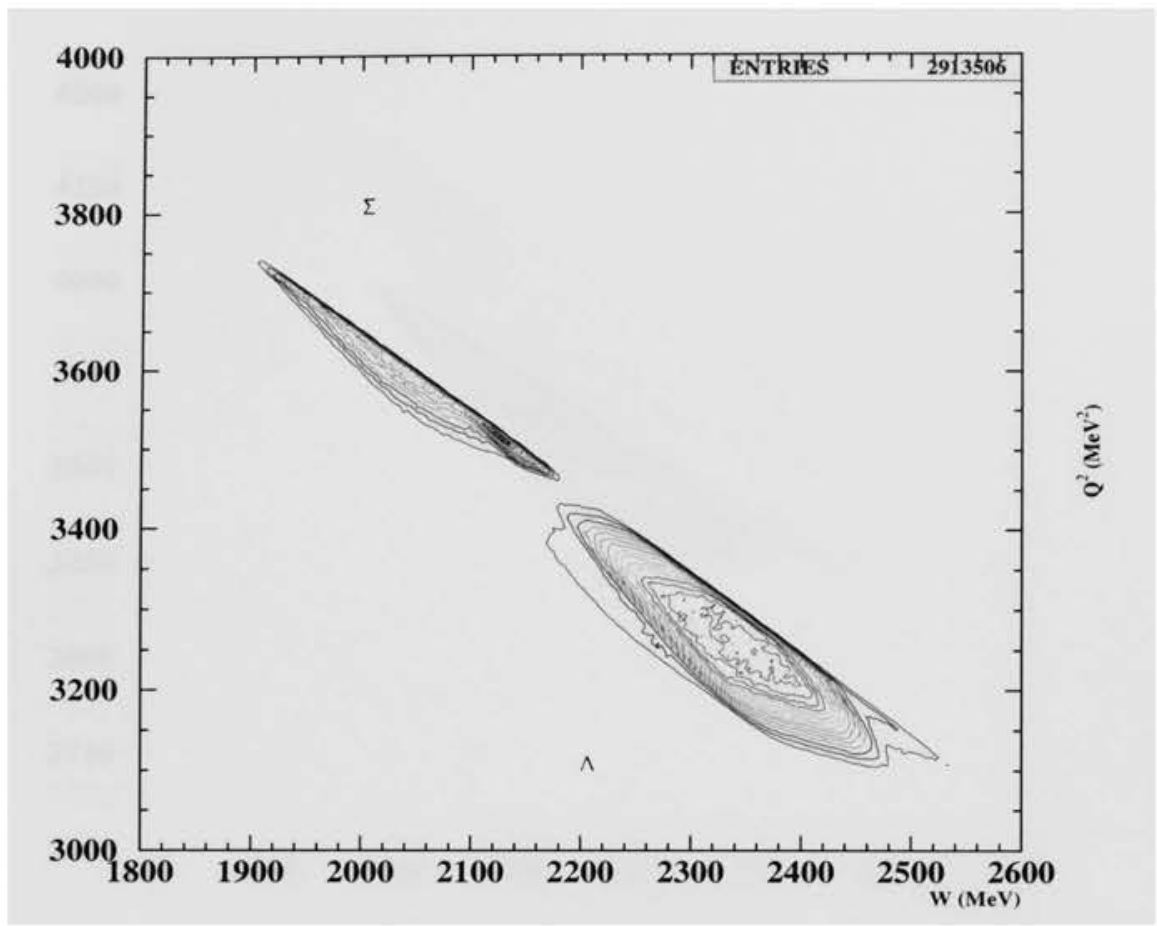

Figure 139: $W$ versus $Q^{2}$ phase space (for both $\Lambda$ and $\Sigma$ channels) for Kin 20 .

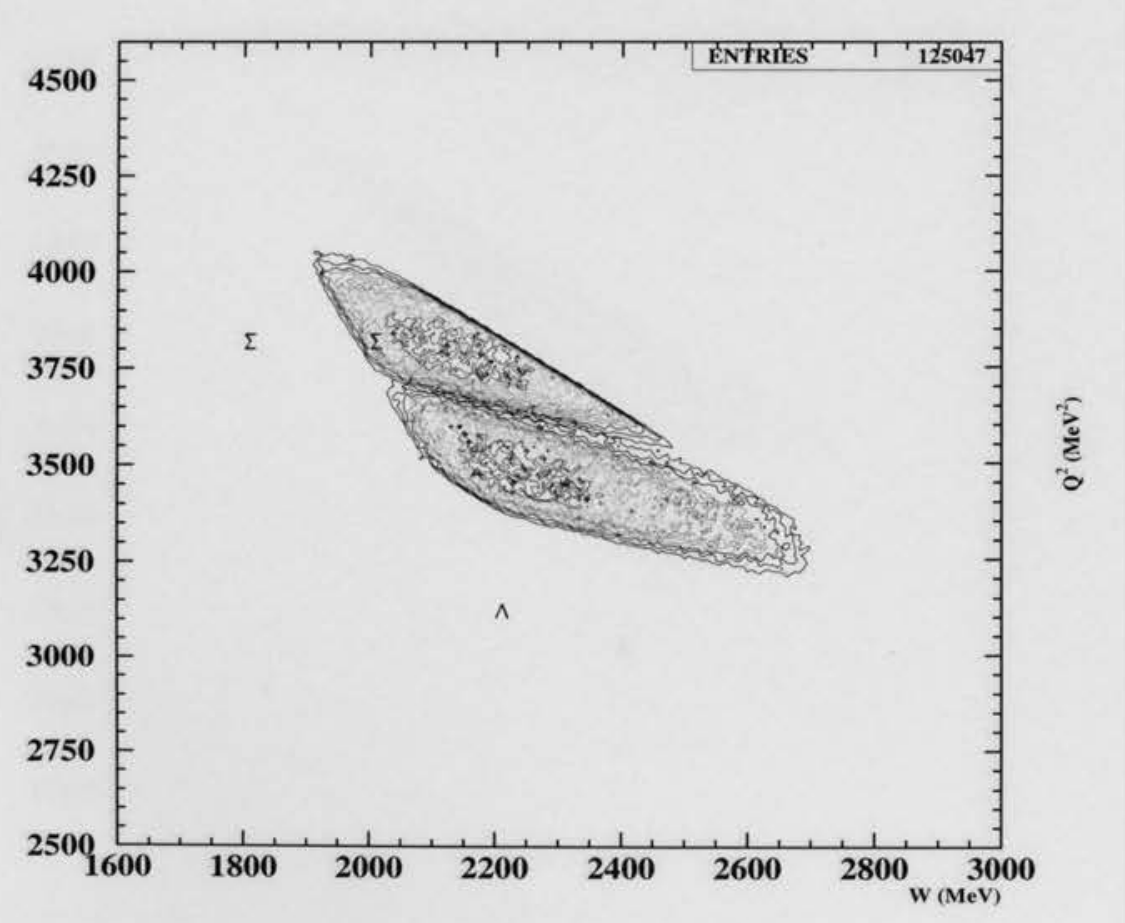

Figure 140: $W$ versus $Q^{2}$ phase space (for both $\Lambda$ and $\Sigma$ channels) for Kin 2 . 


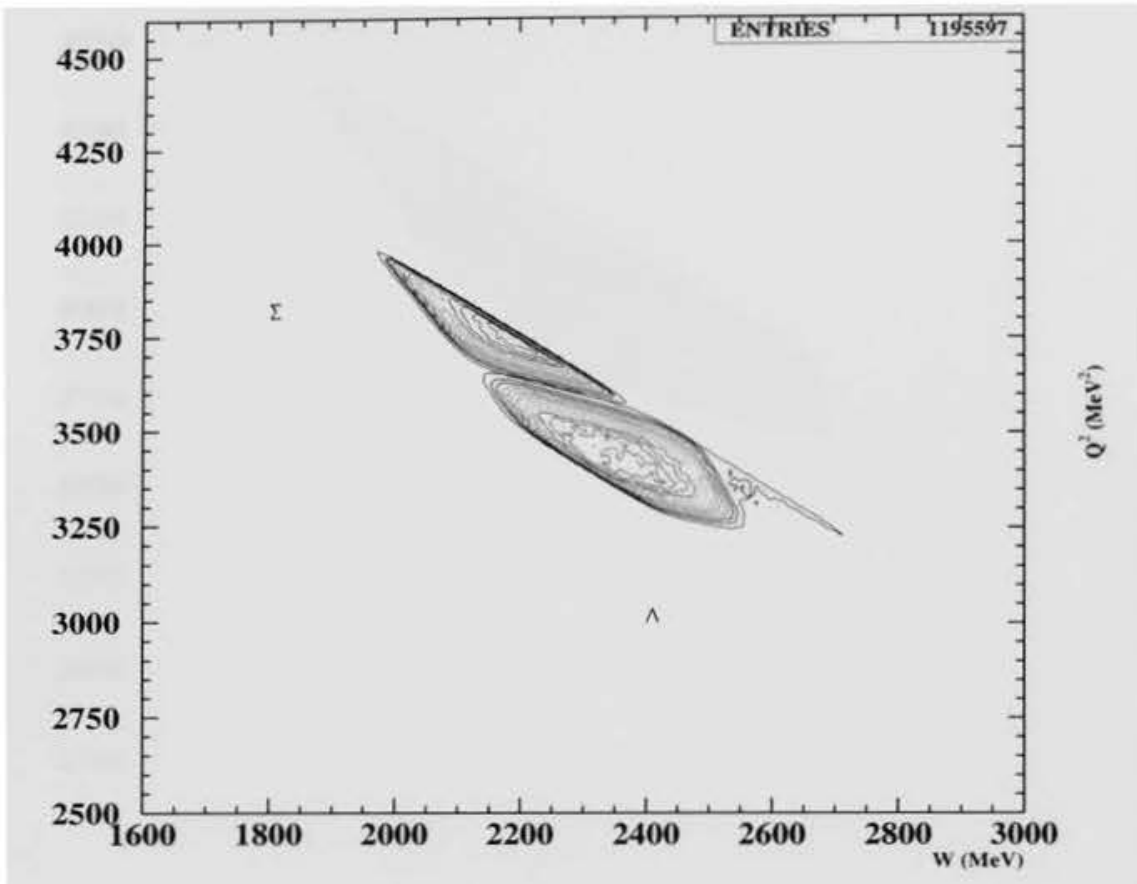

Figure 141: $W$ versus $Q^{2}$ phase space (for both $\Lambda$ and $\Sigma$ channels) for Kin 24 .

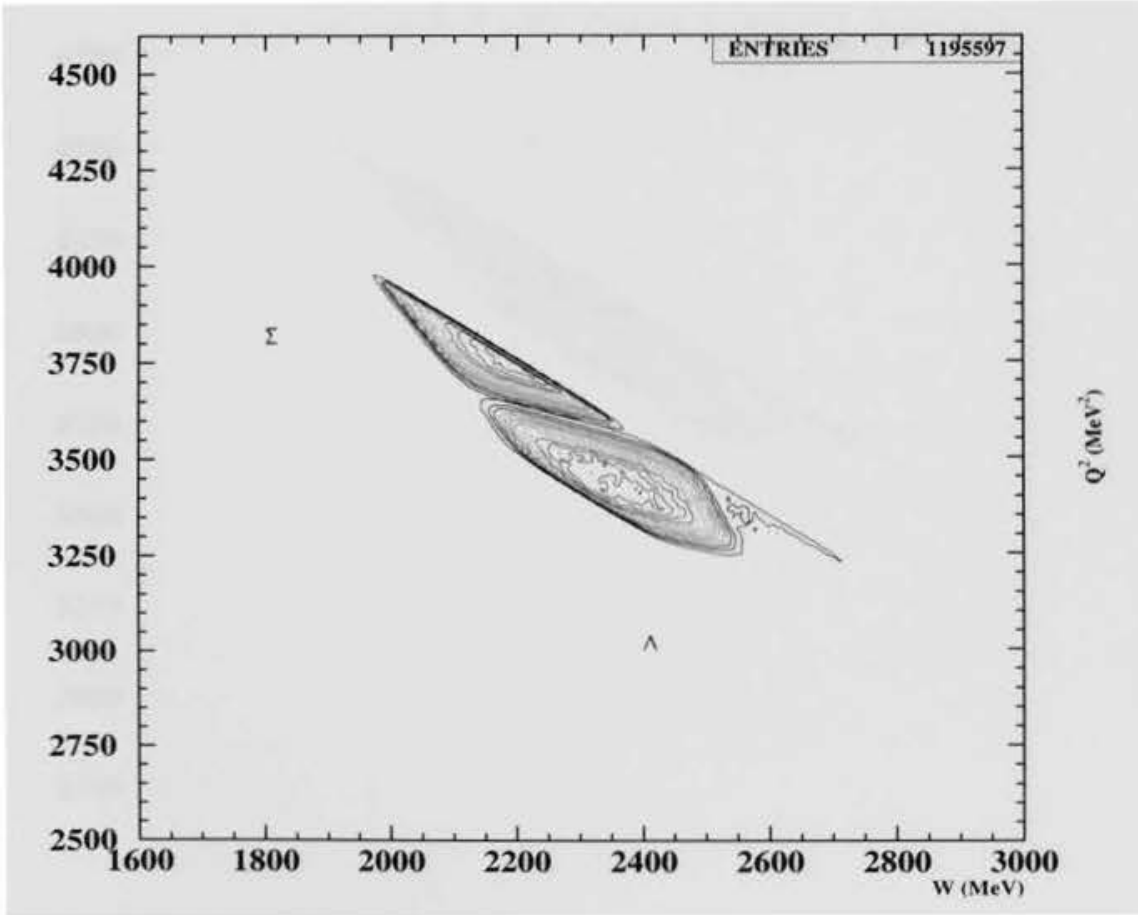

Figure 142: $W$ versus $Q^{2}$ phase space (for both $\Lambda$ and $\Sigma$ channels) for Kin 18 . 


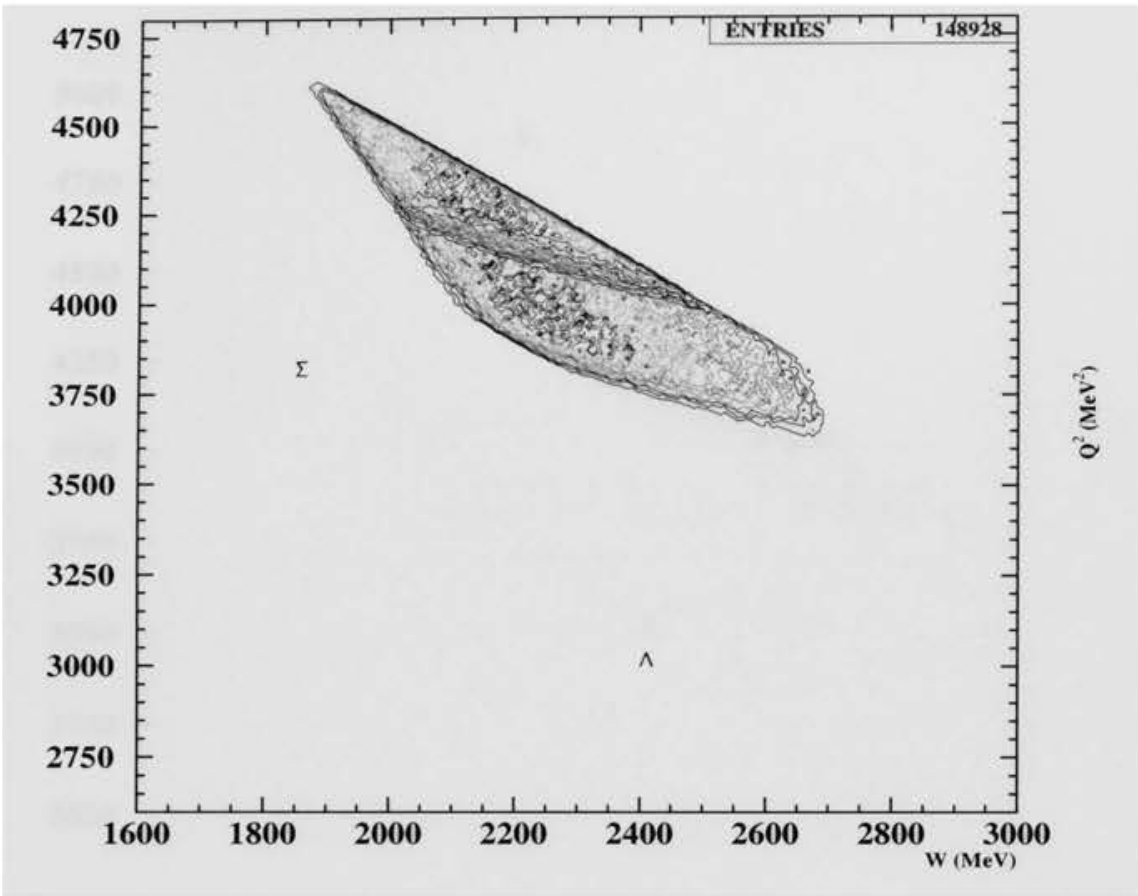

Figure 143: $W$ versus $Q^{2}$ phase space (for both $\Lambda$ and $\Sigma$ channels) for Kin 3 .

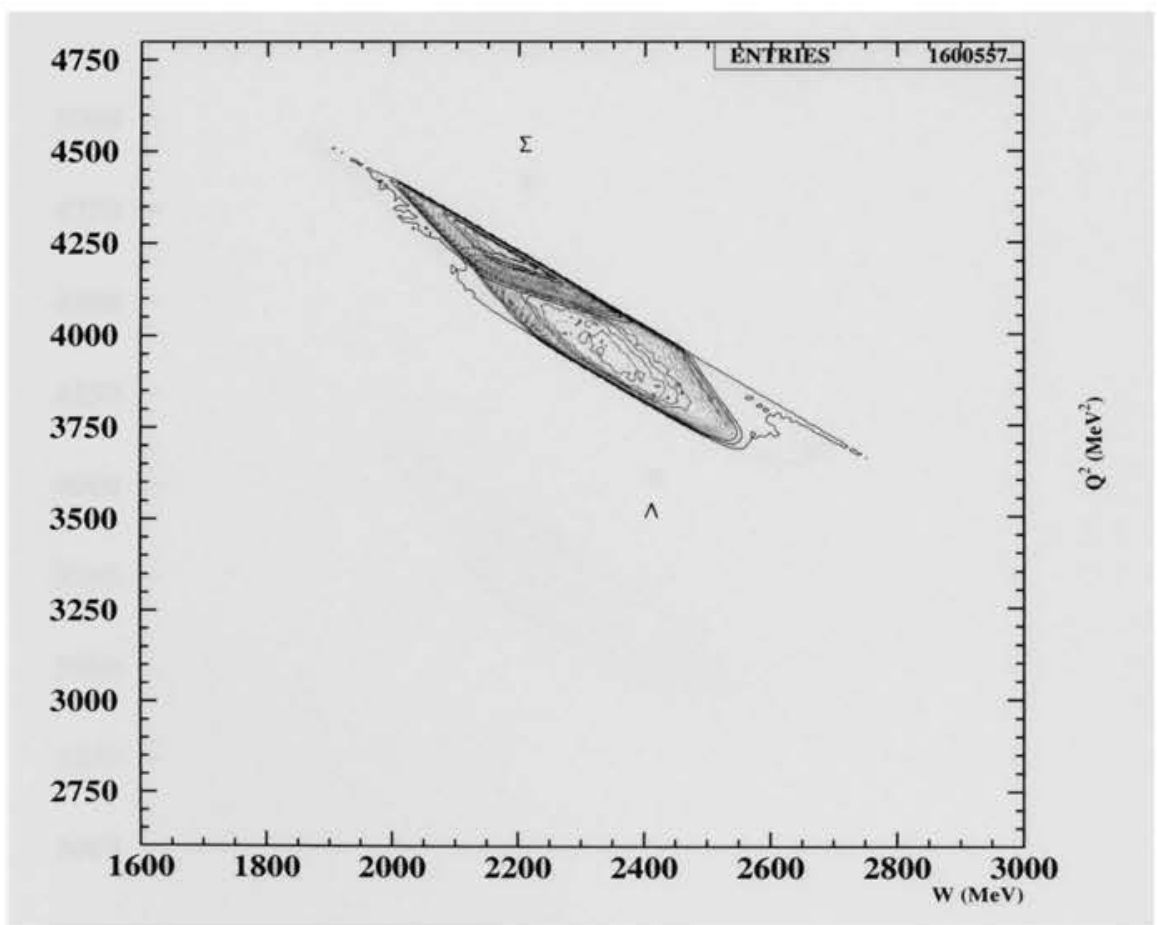

Figure 144: $W$ versus $Q^{2}$ phase space (for both $\Lambda$ and $\Sigma$ channels) for Kin 25 . 


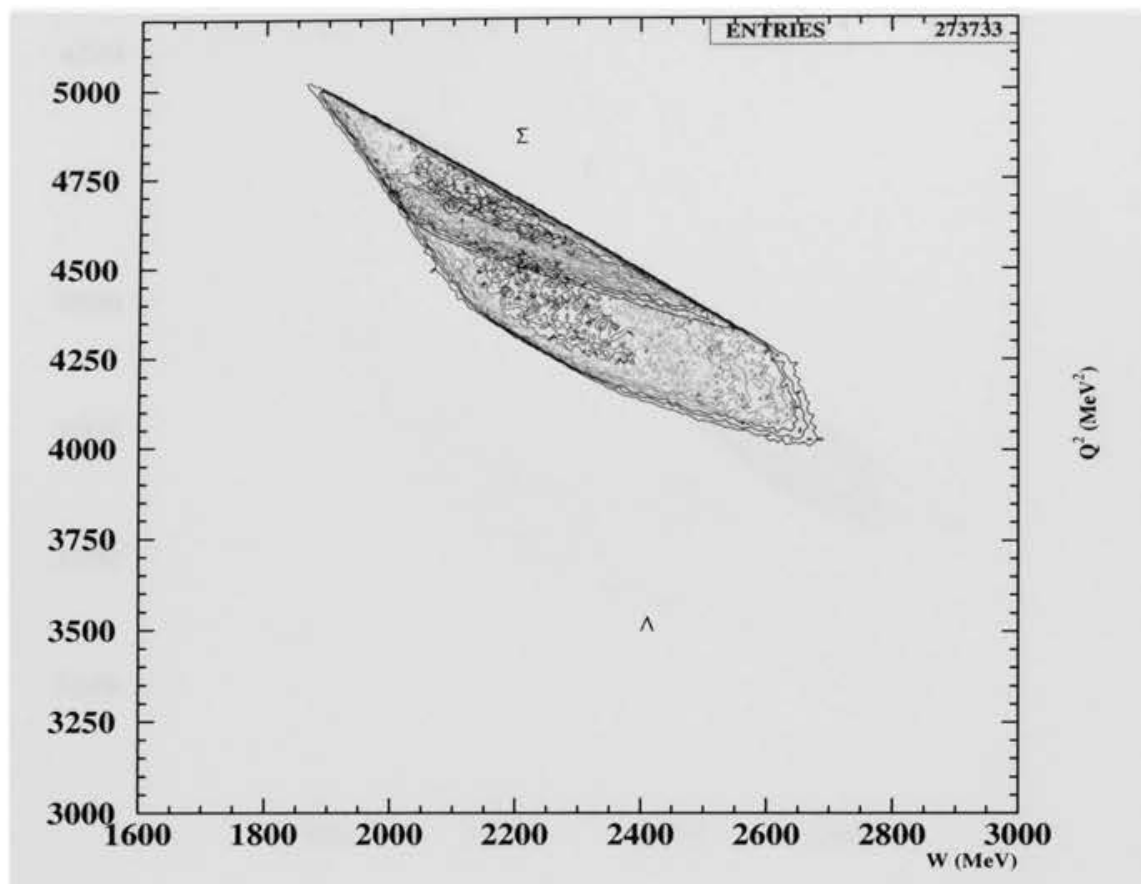

Figure 145: $W$ versus $Q^{2}$ phase space (for both $\Lambda$ and $\Sigma$ channels) for Kin 4 .

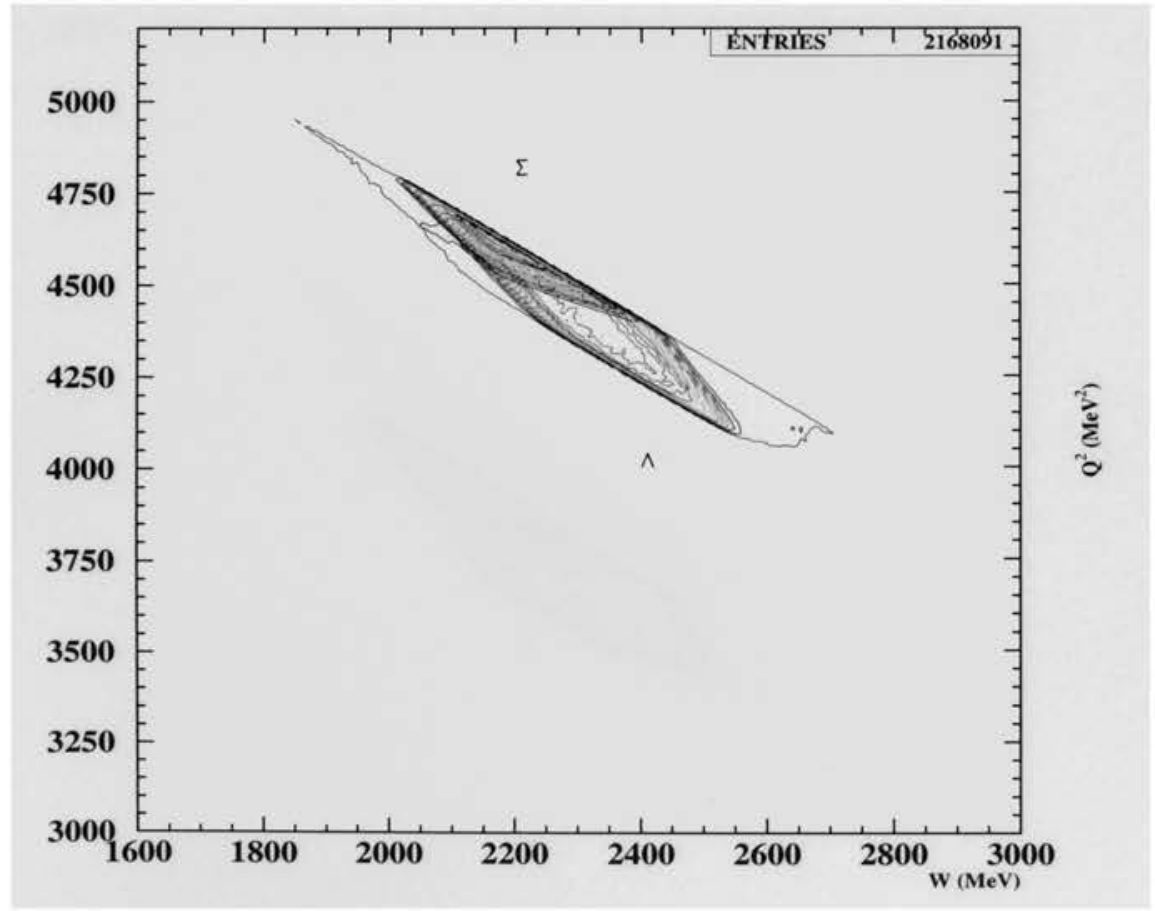

Figure 146: $W$ versus $Q^{2}$ phase space (for both $\Lambda$ and $\Sigma$ channels) for Kin 22 . 


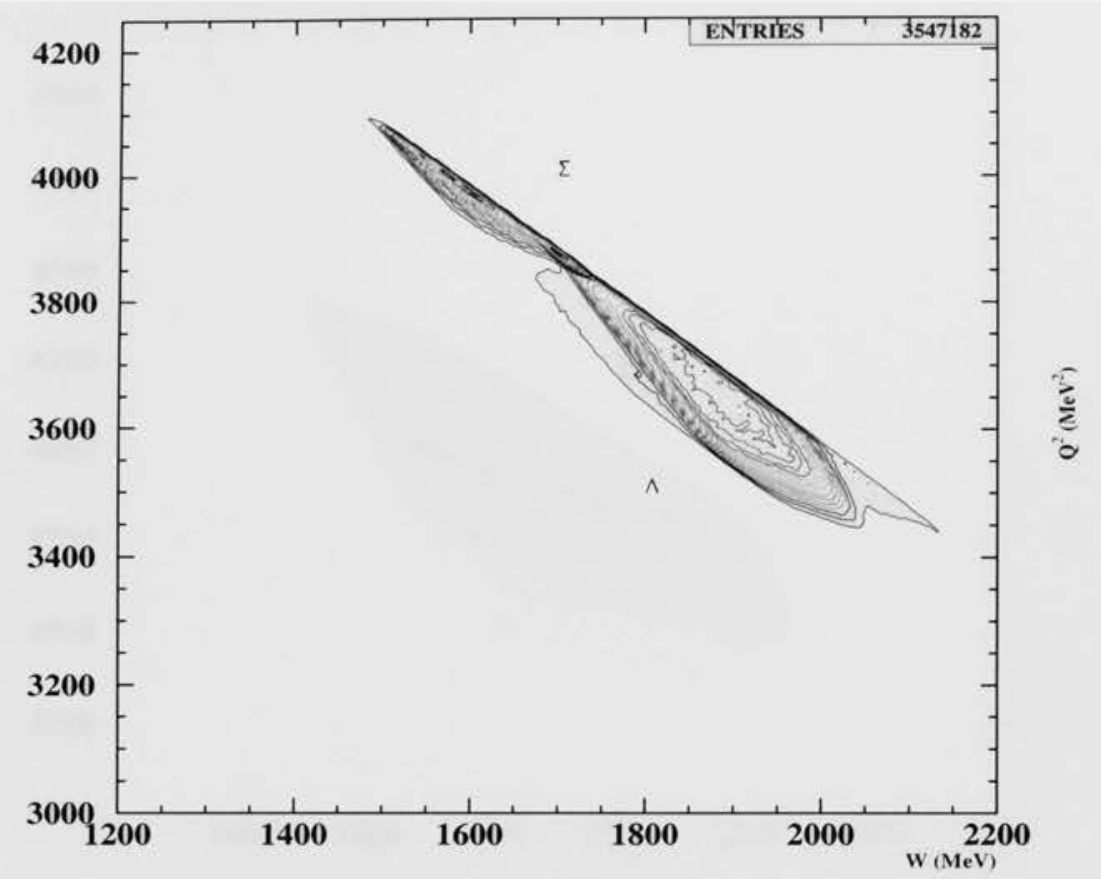

Figure 147: $W$ versus $Q^{2}$ phase space (for both $\Lambda$ and $\Sigma$ channels) for Kin 19.

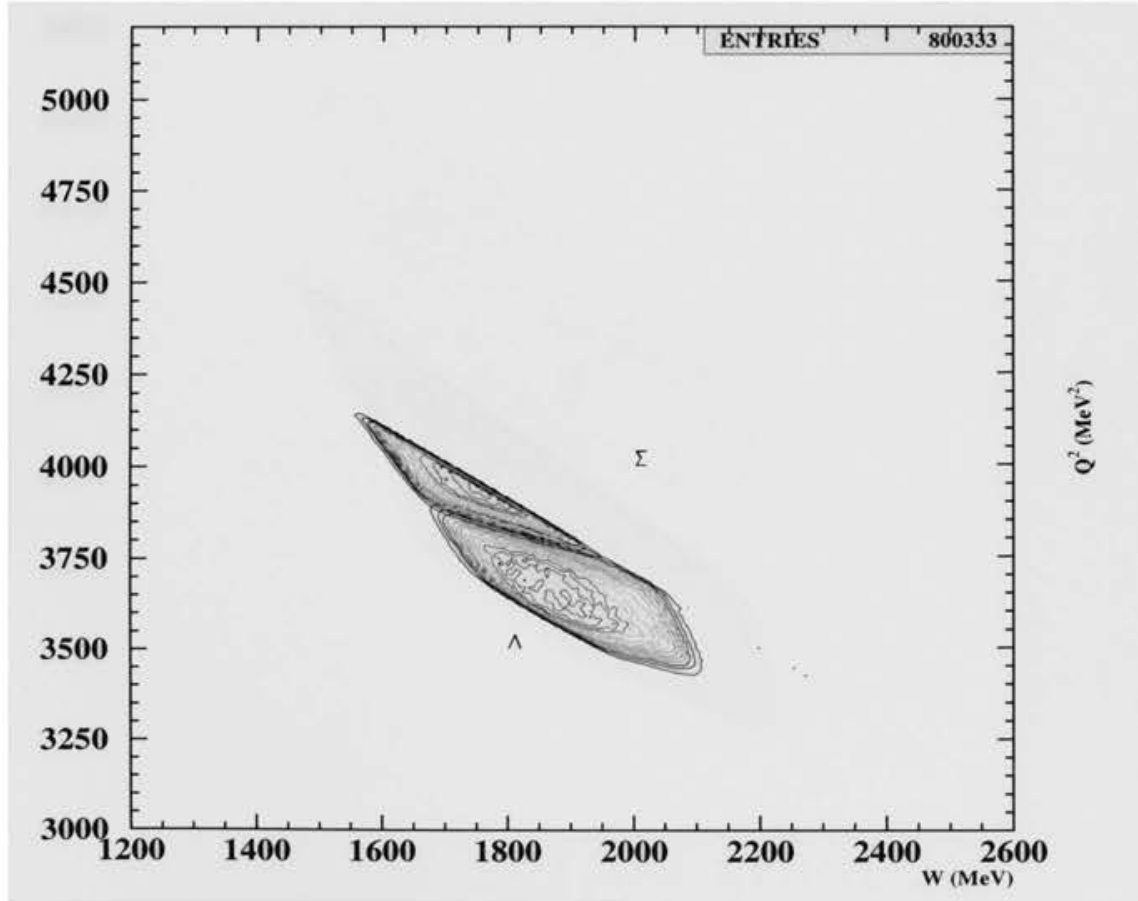

Figure 148: $W$ versus $Q^{2}$ phase space (for both $\Lambda$ and $\Sigma$ channels) for Kin 26 . 


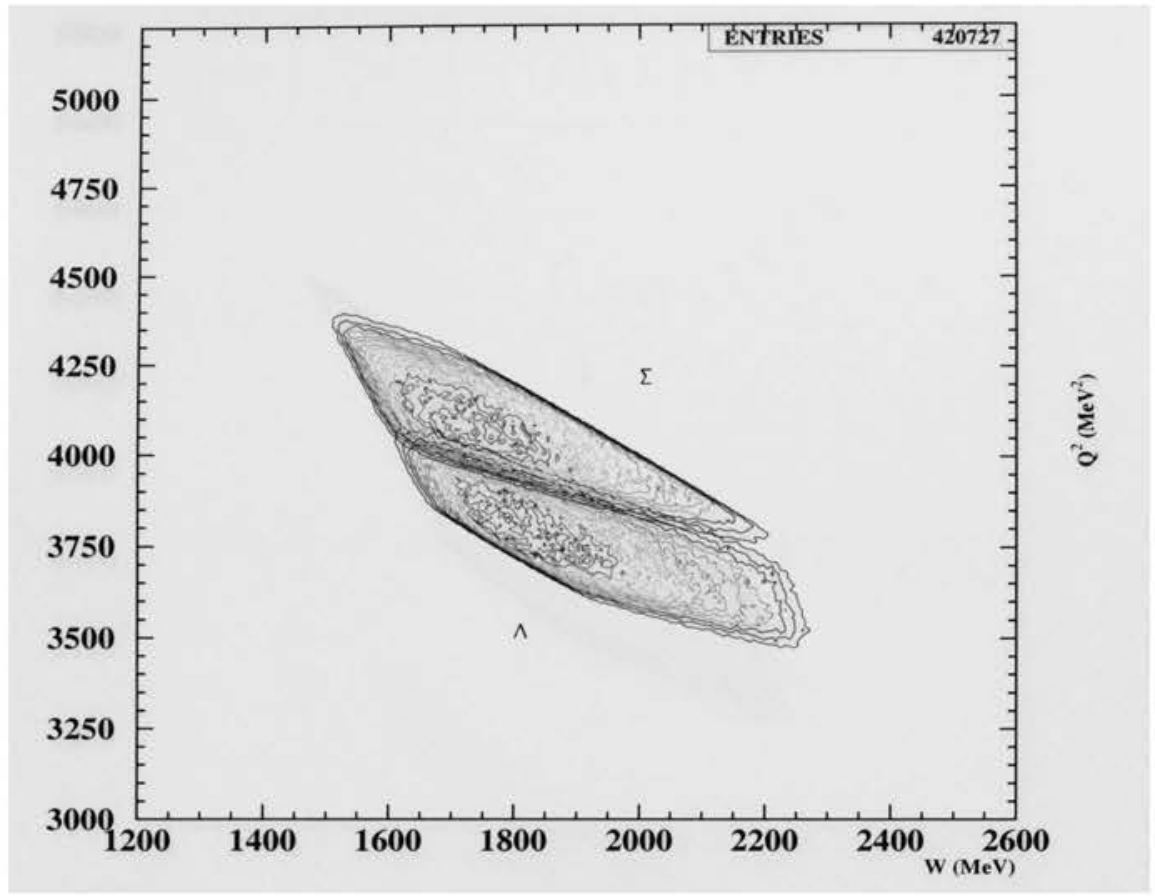

Figure 149: $W$ versus $Q^{2}$ phase space (for both $\Lambda$ and $\Sigma$ channels) for Kin 11 .

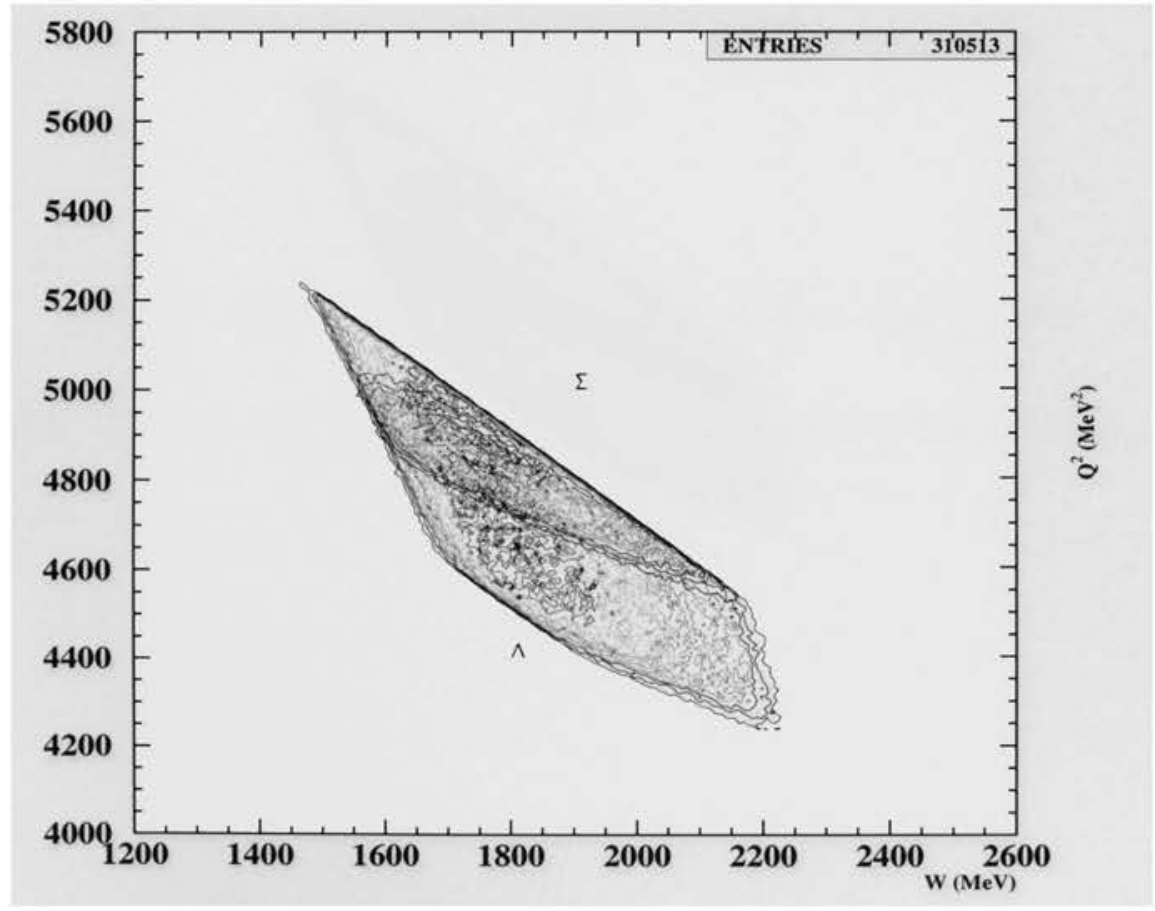

Figure 150: $W$ versus $Q^{2}$ phase space (for both $\Lambda$ and $\Sigma$ channels) for Kin 10 . 


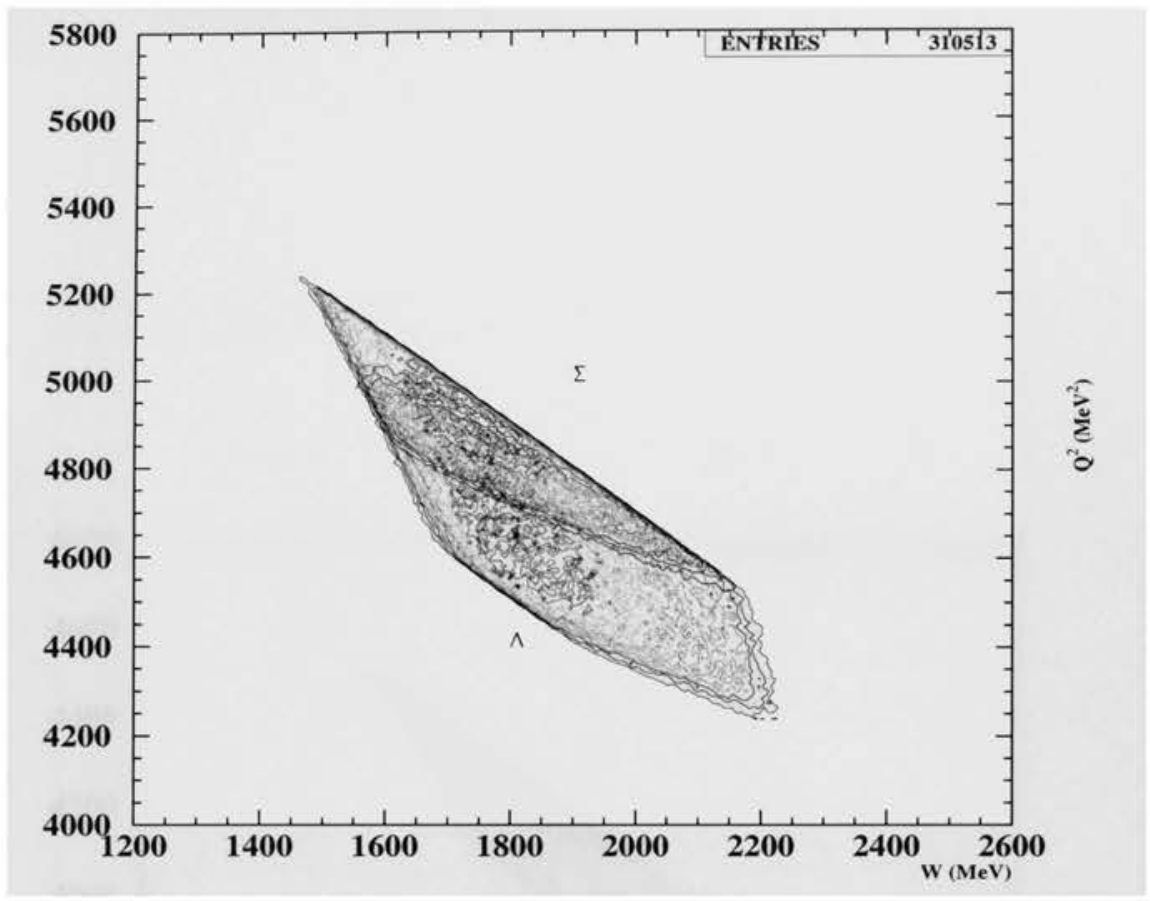

Figure 151: $W$ versus $Q^{2}$ phase space (for both $\Lambda$ and $\Sigma$ channels) for Kin 10 .

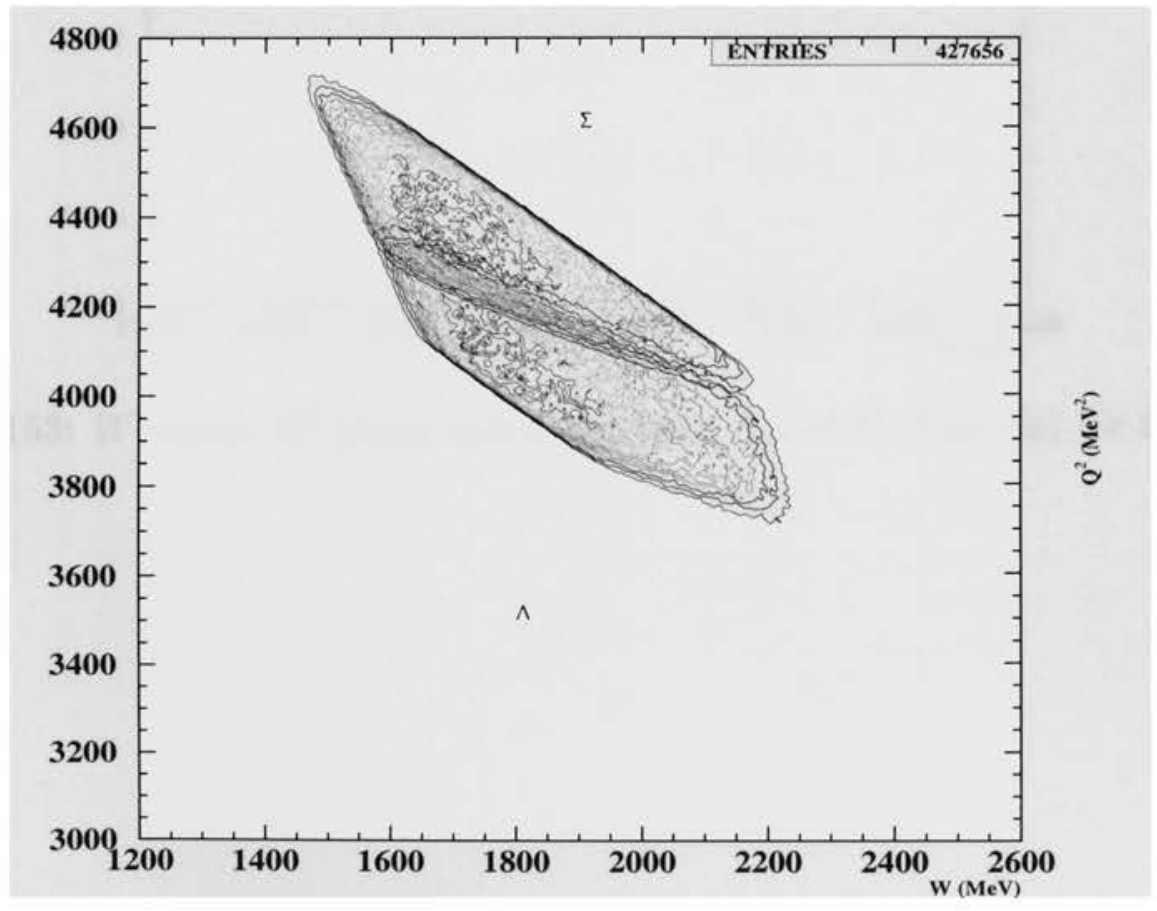

Figure 152: $W$ versus $Q^{2}$ phase space (for both $\Lambda$ and $\Sigma$ channels) for Kin 12 . 


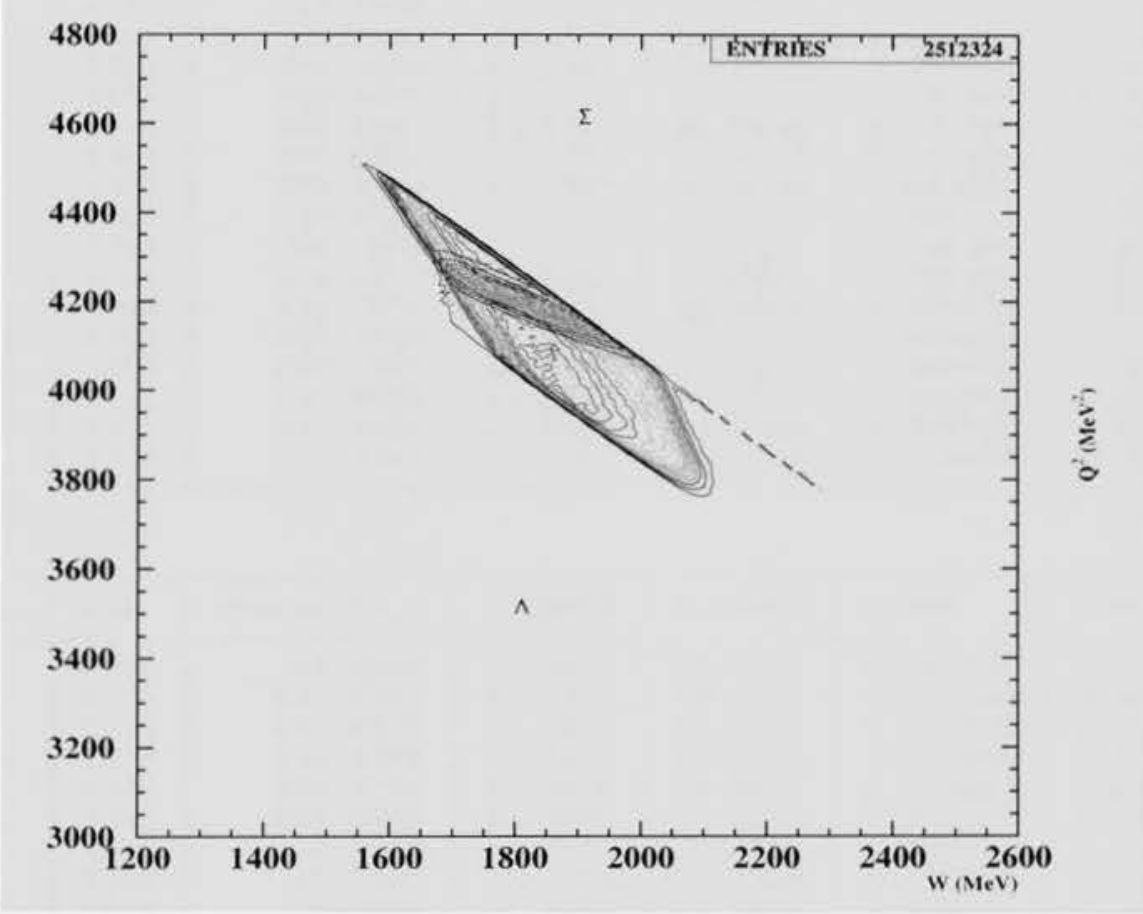

Figure 153: $W$ versus $Q^{2}$ phase space (for both $\Lambda$ and $\Sigma$ channels) for Kin 28 . 


\section{MySQ1 database developed for E98-108 experiment to keep track of parameters specific for each kinematic}

Examples of parameters and their values as extracted from the E981-08 MYSQL database:

\begin{tabular}{|c|c|c|c|c|c|}
\hline run & charge_1x_C & R_ANGLE & L_ANGLE & R_MOM & L_MOM \\
\hline 1736 & 149.9680 & 21.1504 & 22.3715 & 3.077000 & 1.741010 \\
\hline 1737 & 34.7293 & 21.1505 & 22.3715 & 3.077000 & 1.741010 \\
\hline 1738 & 304.1082 & 21.1505 & 22.3715 & 3.077000 & 1.741010 \\
\hline 1739 & 243.5377 & 21.1505 & 22.3715 & 3.077000 & 1.741010 \\
\hline 1740 & 278.0131 & 21.1506 & 22.3716 & 3.077000 & 1.741010 \\
\hline 1741 & 277.9851 & 21.1504 & 22.3715 & 3.077000 & 1.741010 \\
\hline 1742 & 275.6343 & 21.1505 & 22.3716 & 3.077000 & 1.741000 \\
\hline 1743 & 48.9662 & 21.1506 & 22.3714 & 3.077000 & 1.741010 \\
\hline 1750 & 234.7835 & 21.4853 & 21.3528 & 2.981000 & 1.892990 \\
\hline 1751 & 178.3957 & 21.4851 & 21.3527 & 2.981000 & 1.892990 \\
\hline 1752 & 289.2686 & 21.4853 & 21.3528 & 2.981000 & 1.892990 \\
\hline 1753 & 287.7568 & 21.4852 & 21.3528 & 2.981000 & 1.892990 \\
\hline 1754 & 289.7591 & 21.4852 & 21.3528 & 2.981000 & 1.892990 \\
\hline 1755 & 265.6298 & 21.4853 & 21.3529 & 2.981000 & 1.892990 \\
\hline 1757 & 233.6699 & 21.4853 & 21.3528 & 2.981000 & 1.892990 \\
\hline 1758 & 7.7217 & 22.5087 & 18.8064 & 2.719000 & 2.266010 \\
\hline
\end{tabular}

\begin{tabular}{|c|c|c|c|c|c|}
\hline run & charge_1x_C & R_ANGLE & L_ANGLE & R_MOM & L_MOM \\
\hline 3236 & 43.0564 & 37.2807 & 14.6131 & 1.333000 & 2.266010 \\
\hline 3237 & 433.5384 & 37.2807 & 14.6131 & 1.333000 & 2.266000 \\
\hline 3238 & 431.8975 & 37.2806 & 14.6131 & 1.333000 & 2.266000 \\
\hline 3239 & 432.4363 & 37.2807 & 14.6131 & 1.333000 & 2.266000 \\
\hline 3240 & 434.5788 & 37.2806 & 14.6131 & 1.333000 & 2.266000 \\
\hline 3241 & 262.4193 & 37.2806 & 14.6131 & 1.333000 & 2.266000 \\
\hline 3259 & 4.2530 & 31.1653 & 15.4808 & 1.526000 & 2.146990 \\
\hline 3260 & 17.9102 & 31.1652 & 15.4810 & 1.526000 & 2.146990 \\
\hline 3261 & 0.4192 & 31.1652 & 15.4810 & 1.526000 & 2.146990 \\
\hline 3262 & 0.6571 & 31.1652 & 15.4810 & 1.526000 & 2.146990 \\
\hline 3263 & 286.6320 & 31.1653 & 15.4811 & 1.526000 & 2.147000 \\
\hline 3264 & 281.6513 & 31.1653 & 15.4810 & 1.526000 & 2.147000 \\
\hline 3265 & 284.8195 & 31.1652 & 15.4810 & 1.526000 & 2.146990 \\
\hline 3266 & 280.3141 & 31.1652 & 15.4810 & 1.526000 & 2.147000 \\
\hline 3267 & 158.3197 & 31.1653 & 15.4810 & 1.526000 & 2.147000 \\
\hline 3268 & 284.6352 & 31.1653 & 15.4810 & 1.526000 & 2.147000 \\
\hline 3269 & 283.0686 & 31.1653 & 15.4810 & 1.526000 & 2.146990 \\
\hline 3270 & 281.6445 & 31.1652 & 15.4810 & 1.526000 & 2.146990 \\
\hline
\end{tabular}


Examples of parameters and their values as extracted from the E981-08 MySQL database:

\begin{tabular}{|c|c|c|c|c|c|}
\hline run & charge_1x_c & R_ANGLE & L_ANGLE & R_MOM & L_MOM \\
\hline 3271 & 48.6947 & 31.1652 & 15.4810 & 1.526000 & 2.146990 \\
\hline 3290 & 156.4657 & 31.1653 & 15.4810 & 1.526000 & 2.146990 \\
\hline 3291 & 246.0346 & 31.1653 & 15.4810 & 1.526000 & 2.147000 \\
\hline 3292 & 284.3013 & 31.1653 & 15.4810 & 1.526000 & 2.147000 \\
\hline 3293 & 285.0194 & 31.1652 & 15.4809 & 1.526000 & 2.146990 \\
\hline 3294 & 283.8913 & 31.1652 & 15.4810 & 1.526000 & 2.146990 \\
\hline 3295 & 282.5919 & 31.1653 & 15.4810 & 1.526000 & 2.146990 \\
\hline 3296 & 282.4698 & 31.1653 & 15.4810 & 1.526000 & 2.147000 \\
\hline 3451 & 334.5350 & 29.9397 & 16.8198 & 1.650000 & 1.979660 \\
\hline 3452 & 11.8844 & 29.9396 & 21.1455 & 1.650000 & 1.930000 \\
\hline 3453 & 493.7654 & 29.9396 & 21.1455 & 1.650000 & 1.930000 \\
\hline 3454 & 493.5281 & 29.9396 & 21.1455 & 1.650000 & 1.930000 \\
\hline 3455 & 493.4411 & 29.9396 & 21.1455 & 1.650000 & 1.930000 \\
\hline 3456 & 494.4088 & 29.9396 & 21.1455 & 1.650000 & 1.930000 \\
\hline 3457 & 20.6600 & 29.9396 & 21.1455 & 1.650000 & 1.930000 \\
\hline 3458 & 449.6077 & 29.9396 & 21.1455 & 1.650000 & 1.930000 \\
\hline
\end{tabular}

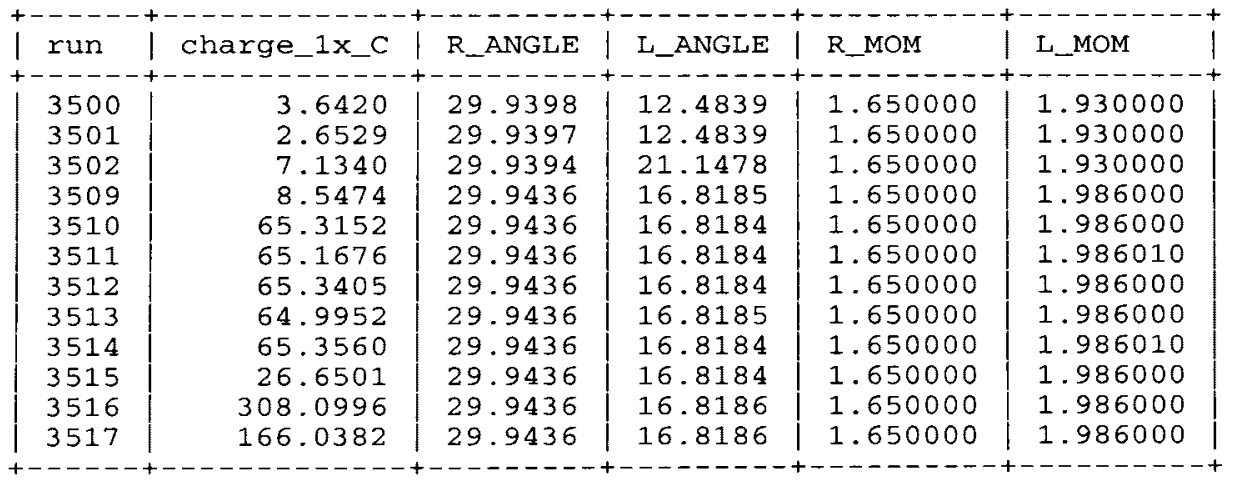


Examples of parameters and their values as extracted from the E981-08 MYSQL database:

\begin{tabular}{|c|c|c|c|c|}
\hline run & R_Misp & L_Misp & RH_LVDT & RV_LVDT \\
\hline $\begin{array}{l}1736 \\
1737 \\
1738 \\
1739 \\
1740 \\
1741 \\
1874 \\
1875 \\
1876 \\
1877 \\
1878 \\
1879 \\
1880 \\
3016 \\
3017 \\
3018 \\
3019 \\
3020\end{array}$ & $\begin{array}{l}-1.631600 \\
-1.637520 \\
-1.644480 \\
-1.649550 \\
-1.651790 \\
-1.624680 \\
-1.737210 \\
-1.735840 \\
-1.738640 \\
-1.740290 \\
-1.636070 \\
-1.598610 \\
-1.610430 \\
-1.023540 \\
-1.027140 \\
-1.030220 \\
-1.036660 \\
-1.019340\end{array}$ & $\begin{array}{l}-1.631600 \\
-1.637520 \\
-1.644480 \\
-1.649550 \\
-1.651790 \\
-1.624680 \\
-1.737210 \\
-1.735840 \\
-1.738640 \\
-1.740290 \\
-1.636070 \\
-1.598610 \\
-1.610430 \\
-1.023540 \\
-1.027140 \\
-1.030220 \\
-1.036660 \\
-1.019340\end{array}$ & $\begin{array}{l}-1053.0000000 \\
-1048.0000000 \\
-1043.0000000 \\
-1039.0000000 \\
-1036.0000000 \\
-1061.0000000 \\
-974.0000000 \\
-975.0000000 \\
-973.0000000 \\
-972.0000000 \\
-1048.0000000 \\
-1081.0000000 \\
-1072.0000000 \\
-1601.0000000 \\
-1598.0000000 \\
-1594.0000000 \\
-1588.0000000 \\
-1605.0000000\end{array}$ & $\begin{array}{l}-194.0000000 \\
-193.0000000 \\
-188.0000000 \\
-183.0000000 \\
-182.0000000 \\
-186.0000000 \\
-222.0000000 \\
-224.0000000 \\
-223.0000000 \\
-222.0000000 \\
-130.0000000 \\
-132.0000000 \\
-119.0000000 \\
500.0000000 \\
500.0000000 \\
505.0000000 \\
514.0000000 \\
483.0000000\end{array}$ \\
\hline run & DT_TRIGG & DT_sca & LH_LVDT & LV_LLVDT \\
\hline $\begin{array}{l}1736 \\
1737 \\
1738 \\
1739 \\
1740 \\
1741 \\
1742 \\
1743 \\
1750 \\
3456 \\
3457 \\
3458 \\
3459 \\
3514 \\
3515 \\
3516 \\
3517\end{array}$ & $\begin{array}{l}-0.00230 \\
-0.00140 \\
-0.00250 \\
-0.00310 \\
-0.00300 \\
-0.00300 \\
-0.00260 \\
-0.00020 \\
-0.00430 \\
0.02570 \\
0.02390 \\
0.02530 \\
0.13810 \\
0.04610 \\
0.04570 \\
0.03920 \\
0.03970\end{array}$ & $\begin{array}{r}0.04250 \\
0.05040 \\
-0.33840 \\
-0.09690 \\
-0.18190 \\
-0.18550 \\
-0.18660 \\
0.01600 \\
-0.05250 \\
0.13890 \\
0.02360 \\
0.24340 \\
0.13510 \\
0.05990 \\
0.05940 \\
-0.14090 \\
0.03910\end{array}$ & $\begin{array}{l}-7227.0000000 \\
-7226.0000000 \\
-7223.0000000 \\
-7223.0000000 \\
-7219.0000000 \\
-7224.0000000 \\
-7217.0000000 \\
-7240.0000000 \\
-7224.0000000 \\
-7945.0000000 \\
-7946.0000000 \\
-7948.0000000 \\
-6922.0000000 \\
-7620.0000000 \\
-7623.0000000 \\
-7603.0000000 \\
-7604.0000000\end{array}$ & $\begin{array}{l}1854.0000000 \\
1855.0000000 \\
1859.0000000 \\
1863.0000000 \\
1863.0000000 \\
1845.0000000 \\
1834.0000000 \\
1859.0000000 \\
1963.0000000 \\
2018.0000000 \\
2019.0000000 \\
2023.0000000 \\
1379.0000000 \\
2211.0000000 \\
2211.0000000 \\
2182.0000000 \\
2178.0000000\end{array}$ \\
\hline
\end{tabular}


Examples of parameters and their values as extracted from the E981-08 MYSQL database:

\begin{tabular}{|c|c|c|c|c|}
\hline run & R_COLI & L_COLL & R_EDT & L_EDT \\
\hline 1736 & 0.6150390 & 0.6170410 & 0.00000000 & 0.00695000 \\
\hline 1737 & 0.6150390 & 0.6169920 & 0.00000000 & 0.00805800 \\
\hline 1738 & 0.6150880 & 0.6170410 & 0.00000000 & 0.00865100 \\
\hline 1739 & 0.6150390 & 0.6170410 & 0.00000000 & 0.00897800 \\
\hline 1740 & 0.6150390 & 0.6170900 & 0.00004100 & 0.01018300 \\
\hline 1741 & 0.6150390 & 0.6169920 & 0.00000000 & 0.00899300 \\
\hline 1742 & 0.6150390 & 0.6170410 & 0.00002100 & 0.01012400 \\
\hline 1743 & 0.6151370 & 0.6170410 & 0.00000000 & 0.00097800 \\
\hline 3160 & 0.6150390 & 0.6169430 & 0.00000000 & 0.01525100 \\
\hline 3161 & 0.6150880 & 0.6169920 & 0.00011500 & 0.01357800 \\
\hline 3162 & 0.6149900 & 0.6168950 & 0.00000000 & 0.01263500 \\
\hline 3163 & 0.6150880 & 0.6168950 & 0.00000000 & 0.01063800 \\
\hline 3164 & 0.6150390 & 0.6169430 & 0.00000000 & 0.01412100 \\
\hline 3165 & 0.6150390 & 0.6169430 & 0.00007940 & 0.01309400 \\
\hline 3512 & 0.6151860 & 0.6170900 & 0.00000000 & 0.00122300 \\
\hline 3513 & 0.6151860 & 0.6169920 & 0.00000000 & 0.00095100 \\
\hline 3514 & 0.6151860 & 0.6170410 & 0.00000000 & 0.00134700 \\
\hline 3515 & 0.6151860 & 0.6169920 & 0.00000000 & 0.00109700 \\
\hline 3516 & 0.6151370 & 0.6170410 & 0.00000000 & 0.01480000 \\
\hline 3517 & 0.6152340 & 0.6170900 & 0.00000000 & 0.01556400 \\
\hline
\end{tabular}




\begin{tabular}{|c|c|c|c|c|}
\hline run & Target & Energy & NMR_right & NMR_left \\
\hline 1736 & $\mathrm{LH} 2-15 \mathrm{~cm}$ & 5614.9399 & 1747840 & 0644292 \\
\hline 1737 & $\mathrm{LH} 2-15 \mathrm{~cm}$ & 5614.8999 & 1.141840 & 0.644291 \\
\hline 1738 & $\mathrm{LH} 2-15 \mathrm{~cm}$ & 5614.9102 & 1.141840 & 0.644291 \\
\hline 1739 & LH2 $-15 \mathrm{~cm}$ & 5614.9702 & 1.141840 & 0.644291 \\
\hline 1740 & LH2 $-15 \mathrm{~cm}$ & 5614.9199 & 1.141840 & 0.644291 \\
\hline 1741 & $\mathrm{LH} 2-15 \mathrm{~cm}$ & 5614.9102 & 1.141840 & 0.644291 \\
\hline 1742 & $\mathrm{LH} 2-15 \mathrm{~cm}$ & 5614.9102 & 1.141840 & 0.644289 \\
\hline 1743 & dum $-15 \mathrm{~cm}$ & 5614.9102 & 1.141840 & 0.644291 \\
\hline 1832 & LH2 $-15 \mathrm{~cm}$ & 5614.9102 & 1.127740 & 0.734896 \\
\hline 1833 & $\mathrm{LH} 2-15 \mathrm{~cm}$ & 5614.9102 & 1.127740 & 0.734887 \\
\hline 1834 & $\mathrm{LH} 2-15 \mathrm{~cm}$ & 5614.9199 & 1.127740 & 0.734889 \\
\hline 1836 & $\mathrm{LH} 2-15 \mathrm{~cm}$ & 5614.9102 & 1.127740 & 0.734895 \\
\hline 1837 & $\mathrm{LH} 2-15 \mathrm{~cm}$ & 5614.9102 & 1.127740 & 0.734896 \\
\hline 1838 & $\mathrm{LH} 2-15 \mathrm{~cm}$ & 5614.9102 & 1.127740 & 0.734896 \\
\hline 1839 & $\mathrm{LH} 2-15 \mathrm{~cm}$ & 5614.9102 & 1.127740 & 0.734895 \\
\hline 1840 & 9 foil-12 & 5614.8999 & 1.127740 & 0.734895 \\
\hline 1841 & 9 foil-12 & 5614.9102 & 1.127740 & 0.714181 \\
\hline 1842 & 9 foil-12 & 5614.9199 & 1.127740 & 0.714181 \\
\hline 1843 & dum $-15 \mathrm{~cm}$ & 5614.9102 & 1.127740 & 0.714180 \\
\hline 1844 & dum $-15 \mathrm{~cm}$ & 5614.9102 & 1.127740 & 0.714181 \\
\hline 1845 & dum $-15 \mathrm{~cm}$ & 5614.9102 & 1.127740 & 0.714181 \\
\hline 3493 & $\mathrm{LH} 2-15 \mathrm{~cm}$ & 4236.9800 & 0.612295 & 0.714182 \\
\hline 3494 & LH2 $-15 \mathrm{~cm}$ & 4236.9702 & 0.612295 & 0.714182 \\
\hline 3495 & LH $2-15 \mathrm{~cm}$ & 4236.9600 & 0.612295 & 0.714181 \\
\hline 3496 & LH $2-15 \mathrm{~cm}$ & 4236.9800 & 0.612295 & 0.714181 \\
\hline 3497 & LH2 $-15 \mathrm{~cm}$ & 4236.9800 & 0.612295 & 0.714181 \\
\hline 3498 & 9 foil-12 & 4236.9600 & 0.612295 & 0.714182 \\
\hline 3499 & 9 foil -12 & 4237.0000 & 0.612295 & 0.714183 \\
\hline 3500 & 9 foil -12 & 4236.9600 & 0.612295 & 0.714183 \\
\hline 3512 & dum-15cm & 4236.9702 & 0.612294 & 0.734893 \\
\hline 3513 & $\mathrm{dum}-15 \mathrm{~cm}$ & 4236.9600 & 0.612295 & 0.734893 \\
\hline 3514 & dum $-15 \mathrm{~cm}$ & 4236.9702 & 0.612294 & 0.734893 \\
\hline 3515 & dum $-15 \mathrm{~cm}$ & 4237.0601 & 0.612294 & 0.734894 \\
\hline 3516 & LH $2-15 \mathrm{~cm}$ & 4237.0000 & 0.612295 & 0.734894 \\
\hline 517 & $\mathrm{LH} 2-15 \mathrm{~cm}$ & 4237.0200 & 0.612295 & 0.734893 \\
\hline
\end{tabular}




\section{J E98-108 experiment: Setup for object oriented analysis over the web}

This webpage at: http://barfly.fiu.edu/kaonexp/root.htm QUESTIONS:Marius Coman MCajlab.org

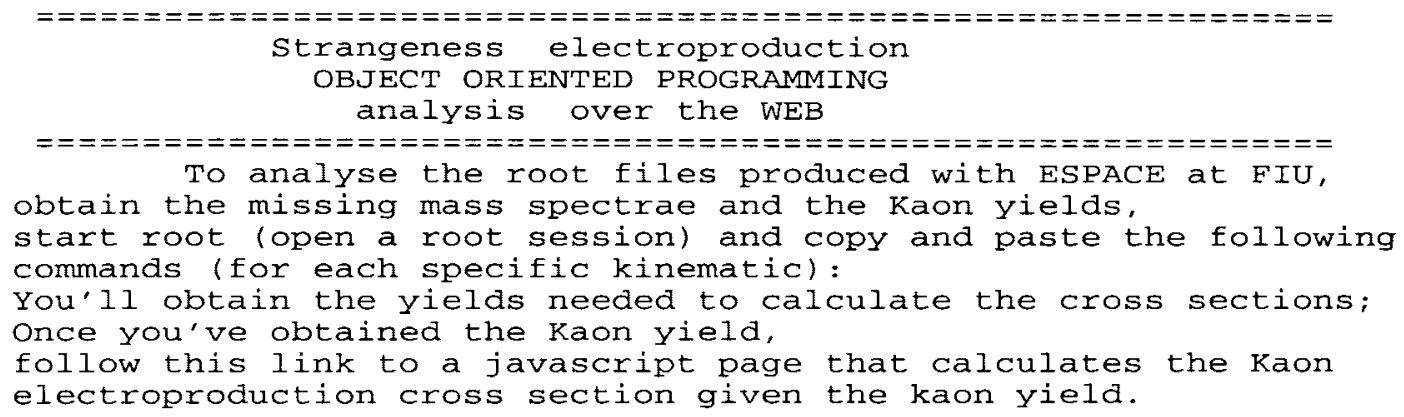

For kinematic 1 :

TWebFile kin1 ("http://barfly.fiu.edu/kaonexp/kinematicsl.root") ; TH1F *h1001 = new TH1F("h1001", "Kaons KIN 1",350,950..1300.); h1001->Add (h11, h15, 1, -.3); 


\section{K E98-108 experiment: Sensitivity of the efficiencies of the scintillator detector to gas Črenkov}

\begin{tabular}{|c|c|c|c|c|c|c|c|c|}
\hline$\overline{R u n}$ & $S 1_{E}$ & $\sigma$ & $S 2_{E}$ & $\sigma$ & $S 1_{E_{N o}}$ & $\sigma$ & $S 2_{E_{\text {NoGas }}}$ & 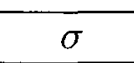 \\
\hline 1742 & 0.9912 & 0004 & 0.9907 & 00 & & $\overline{00}$ & & 00 \\
\hline 1743 & 1.000 & & 1.000 & & & & .000 & 075 \\
\hline 1744 & $\overline{0116}$ & 0.026 & 0.0132 & 0.026 & 1.000 & .0022 & .000 & .0022 \\
\hline 1745 & 000 & 0.002 & 1.000 & 0.002 & 1.000 & 9 & & \\
\hline 1746 & 000 & 2 & .000 & 0002 & 0.9937 & & 00 & $\overline{002}$ \\
\hline 1747 & 000 & & 1.000 & & 1.000 & .0002 & 0.9927 & 002 \\
\hline 1748 & 000 & 0003 & 1.000 & .0003 & 1.000 & .0002 & 1.000 & $\overline{0.0002}$ \\
\hline 749 & 000 & & 1.000 & 35 & 1.000 & 31 & 1.000 & .0031 \\
\hline 1750 & 9922 & & 9922 & & 0.9917 & & 0.9913 & $\overline{004}$ \\
\hline 1751 & 9961 & 005 & 9961 & .0005 & 0.9958 & 0.0004 & 0.9958 & .0004 \\
\hline 1752 & 9885 & 04 & .9895 & 904 & 9875 & .0003 & 0.9878 & 0.0003 \\
\hline 1753 & 89 & & 94 & & & & 893 & \\
\hline 1754 & 987 & 4 & 0.9885 & 0.0004 & 9867 & 0.0003 & 0.9878 & 0.0003 \\
\hline 1755 & 9833 & 4 & 0.9833 & 0.0 & 53 & 0.0003 & 0.9857 & .0003 \\
\hline 1756 & & & & & & & 1 & \\
\hline 1757 & 863 & & 45 & & 0. & .0004 & 0.9835 & .0004 \\
\hline 1758 & 0 & 2 & 1.000 & .0002 & 1.000 & 0.0002 & $\overline{000}$ & 0.0002 \\
\hline 1759 & & & & & - & & .000 & .0018 \\
\hline 1760 & 962 & & & & .9954 & 08 & 9954 & .0008 \\
\hline 1761 & 844 & 4 & 844 & 04 & 9861 & 03 & 0.9864 & 0.0003 \\
\hline 1762 & 47 & & & & 56 & & 0.9866 & 0.0003 \\
\hline 1763 & 97 & 4 & 9897 & 004 & 989 & 503 & 0.9887 & .0003 \\
\hline 1764 & 889 & & 889 & & & & 67 & 0.0003 \\
\hline 1765 & 392 & & 9881 & & 0.9879 & 0.0003 & 0.9875 & 0.0003 \\
\hline 1766 & & & 92 & 04 & 0.987 & 0.0003 & 0.988 & 0.0003 \\
\hline 1767 & 877 & 4 & 366 & & & & 00 & 0.0003 \\
\hline 1768 & 00 & 2 & 0 & 02 & 0.9966 & 0.0001 & 0.9968 & 0.0001 \\
\hline 1769 & & & & & 1.000 & 0.0001 & 1.000 & 0.0001 \\
\hline 1770 & 00 & & & & 1.000 & & 1.000 & 0.0003 \\
\hline 1771 & 882 & 4 & 39 & & 0.9871 & 0.0003 & 0.9865 & 0.0003 \\
\hline 1772 & & & & & 0.9917 & 0.0003 & 0.9924 & 0.0003 \\
\hline 1797 & 1.000 & & 00 & & 00 & 01 & 1.000 & 0.0001 \\
\hline 1798 & 1.000 & & 1.000 & & 1.000 & 0.0001 & 1.000 & 0.0001 \\
\hline 1799 & & & 0.9927 & & 0.9966 & 0.001 & 0.9966 & 0.001 \\
\hline 1800 & 855 & & 0.9827 & 09 & 0.9889 & 0.0006 & 0.9875 & 0.0006 \\
\hline 1801 & & & 0.992 & .0008 & 0.9838 & 0.0006 & 0.9851 & 0.0006 \\
\hline 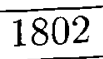 & 1.000 & 0.00 & 1.000 & 0.001 & 0.9846 & 0.0009 & 0.9877 & 0.000 \\
\hline
\end{tabular}




\begin{tabular}{|c|c|c|c|c|c|c|c|c|}
\hline Run & $S 1_{E}$ & $\sigma$ & $S 2_{E}$ & $\sigma$ & $S 1_{E_{N o C}}$ & $\sigma$ & $S 2_{E_{N o}}$ & - \\
\hline 1803 & 0.8255 & .0014 & 0.8368 & 0014 & & 0007 & & .0007 \\
\hline 1804 & 0.985 & 0.001 & 0.985 & & 896 & 13 & 9899 & 0003 \\
\hline 1805 & 9907 & 0005 & .9907 & .0005 & 0.9801 & 2 & 3 & 0002 \\
\hline 1806 & 9951 & 0011 & 0.9951 & .0011 & .9877 & 0007 & 9877 & .0007 \\
\hline 1807 & .000 & & 000 & 0022 & 9915 & & .9915 & .0015 \\
\hline 1808 & 0.9853 & & 0.9879 & .0004 & 0.986 & .0003 & 0.9875 & .0003 \\
\hline 1809 & 0.9805 & .0005 & 0.9797 & 0.0005 & 0.9828 & .0003 & 002 & \\
\hline 1810 & 0.9788 & .0005 & 0.9804 & .0005 & 0.9815 & .0003 & .9827 & .0003 \\
\hline 1811 & 9833 & 5 & 0.9825 & 5 & 0.9824 & & .9844 & $\overline{03}$ \\
\hline 1812 & 9741 & 001 & 0.9741 & 0.001 & 0.9826 & .0007 & .9809 & .0007 \\
\hline 1813 & 9838 & 0005 & 0.9847 & 5 & 63 & 03 & .9867 & .0003 \\
\hline 1814 & 99 & & 0.9875 & & 85 & & 893 & \\
\hline 1815 & 00 & & 1.000 & 2 & 1.000 & & 1.000 & .0001 \\
\hline 1816 & 500 & 2 & 1.000 & 2 & 50 & 01 & 00 & 0001 \\
\hline 1817 & 00 & & 00 & & 00 & & & \\
\hline 1818 & 50 & & & & 000 & & 00 & \\
\hline 1819 & 00 & 2 & 1.000 & & 1.000 & & 1.000 & 01 \\
\hline 1820 & 818 & 04 & 2 & 14 & 24 & 34 & 811 & 04 \\
\hline 1821 & 325 & & 31 & & & & 847 & \\
\hline 1822 & 87 & & 87 & & 47 & & 852 & \\
\hline 1823 & 827 & 6 & 38 & 6 & 39 & & 848 & .0005 \\
\hline 824 & 00 & & & & & & 0 & \\
\hline 1825 & 974 & & & & & & 356 & 04 \\
\hline 1826 & 867 & 4 & 61 & & & & 518 & 0.0008 \\
\hline 827 & 907 & & & & 61 & & 894 & 0.0004 \\
\hline 28 & 4 & & & & & & 961 & 0.0004 \\
\hline 1829 & 815 & & & & & & & 0.0004 \\
\hline 1830 & 84 & & & & 45 & & .9841 & 0.0004 \\
\hline 1831 & & & & & & & 845 & .0003 \\
\hline 1832 & 847 & & & & & & 70 & 0.0003 \\
\hline 1833 & 849 & 03 & 69 & & 372 & 03 & 0.9889 & 0.0003 \\
\hline 1834 & 835 & & & & & & 0.9848 & 0.0003 \\
\hline 337 & 87 & & 8875 & & 9844 & & 0.985 & 0.0003 \\
\hline 1838 & 865 & & 74 & & .9859 & 0.0003 & 0.9866 & 0.0003 \\
\hline 1839 & & & & & & & 0.9849 & 0.0003 \\
\hline 1840 & & & 00 & & 000 & & 1.000 & 0.0001 \\
\hline 1841 & & & & & 1.000 & & 1.000 & 0.0002 \\
\hline 1842 & & & & & 1.000 & & 1.000 & 0.0002 \\
\hline 1843 & & & & & 1.000 & 02 & 1.000 & 0.0002 \\
\hline & & & & & 1.000 & & 1.000 & 0.0002 \\
\hline & 1.000 & 0.0002 & 1.000 & & 1.000 & 0.0002 & 1.000 & 0.0002 \\
\hline
\end{tabular}




\begin{tabular}{|c|c|c|c|c|c|c|c|c|}
\hline Run & $S 1_{E}$ & $\sigma$ & $S 2_{E}$ & $\sigma$ & $1_{E_{N o G a}}$ & $\sigma$ & $2_{E_{N o G a s}}$ & 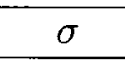 \\
\hline 1846 & 1.000 & .0002 & 1.000 & 0002 & & .0002 & & .0002 \\
\hline 1847 & 1.000 & 0002 & 1.000 & & 0.9872 & 0005 & $n$ & 0005 \\
\hline $18 \overline{48}$ & $\overline{9914}$ & $\overline{0006}$ & 0.9914 & 0.0006 & 0.9998 & .0002 & 9989 & .0002 \\
\hline 1849 & 9902 & 0005 & .9902 & 0.0005 & 0.9912 & .0005 & 9992 & .0005 \\
\hline 50 & & 0005 & 9838 & 0005 & 833 & 0005 & 9817 & .0005 \\
\hline 1851 & 871 & & 0.9892 & 0.0005 & 0.9843 & 0.0005 & .9866 & .0005 \\
\hline 1852 & .9871 & .0005 & 0.9892 & 0.0005 & 0.9851 & .0005 & 9867 & .0005 \\
\hline 1856 & 0.994 & 0009 & 0.994 & 0.0009 & 0.9869 & .0008 & .9869 & .0008 \\
\hline 1857 & 82 & 0004 & 9831 & 0.0004 & 0.9839 & .0003 & 9843 & .0003 \\
\hline 1858 & 814 & 0002 & 9824 & 0.0002 & 0.981 & 0.0002 & 9816 & .0002 \\
\hline 1859 & .9877 & .0005 & .9855 & 0.0 & 0.9844 & .0005 & .9836 & .0005 \\
\hline 1860 & 9832 & 4 & .9832 & 0.0004 & 0.9846 & $\overline{04}$ & 846 & \\
\hline 1861 & 100 & 01 & 00 & & .000 & & 1.000 & .0001 \\
\hline 1862 & 00 & - & 0 & - & 00 & 8 & 00 & 0008 \\
\hline 63 & 000 & 1 & 00 & & 000 & & 00 & \\
\hline 1864 & 00 & & & & 000 & & 000 & \\
\hline 1865 & 1.000 & 4 & 1.000 & 0.0 & 1.000 & 0.0 & 000 & $\overline{0003}$ \\
\hline 66 & 00 & 3 & 00 & & 0 & 02 & 00 & 002 \\
\hline 1867 & 00 & & 00 & & 00 & & 1.000 & 002 \\
\hline 1868 & 944 & & 944 & & 996 & & 9.996 & 0.001 \\
\hline 1869 & 00 & 6 & 84 & & 79 & 05 & 89 & .0005 \\
\hline 1870 & 379 & & & & & & $\sqrt{32}$ & \\
\hline 1871 & 771 & & 42 & & 99 & & 93 & 01 \\
\hline 1872 & 868 & 6 & 68 & 6 & 9892 & .0005 & 9978 & 0.0005 \\
\hline 1873 & 782 & & & & 05 & & 828 & 0.0006 \\
\hline 1874 & & & & & & & 856 & 0005 \\
\hline 75 & & & & & & & 932 & 0.0006 \\
\hline 1876 & 19 & & & & 99 & 55 & 0.9799 & 0.0005 \\
\hline 1877 & & & & & 55 & & 855 & .0005 \\
\hline 878 & 835 & & & & 344 & & & 0.0002 \\
\hline 1879 & 867 & & 69 & & 9867 & 0.0002 & 0.9868 & 0.0002 \\
\hline 1880 & & & & & & & 1.000 & 0.0001 \\
\hline 81 & & & & & 00 & 01 & 1.000 & 0.0001 \\
\hline 882 & & & 00 & 2 & 00 & 0.0001 & 1.000 & 0.0001 \\
\hline 1883 & & & & & & & 1.000 & 0.0001 \\
\hline 384 & 8 & & 8998 & 14 & 9848 & 03 & 0.9853 & 0.0003 \\
\hline 1885 & & & & & 0.9859 & & 0.9853 & 0.0003 \\
\hline 1886 & & & 8857 & & 9863 & & 0.9869 & 0.0003 \\
\hline 1887 & 53 & & 64 & & 0.9854 & 0.0003 & 0.9859 & 0.0003 \\
\hline & & & & & & & 1.000 & 0.0365 \\
\hline 000 & & & 0.9914 & 0.6001 & 0.0010 & 0.0003 & 0.9881 & 0.0003 \\
\hline
\end{tabular}




\begin{tabular}{|c|c|c|c|c|c|c|c|c|}
\hline Run & $S 1_{E}$ & $\sigma$ & $S 2_{E}$ & $\sigma$ & $S 1_{E_{\text {NoGas }}}$ & $\sigma$ & $S 2_{E_{N o G}}$ & 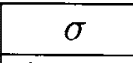 \\
\hline 1889 & 0.9885 & 0.0004 & 0.9868 & 0.0004 & & 0.0003 & & 0.0003 \\
\hline 1889 & .9863 & .0004 & 0.9869 & 0.0004 & 868 & 0.0003 & 9876 & 0.0003 \\
\hline 1890 & 9994 & .0004 & 0.9994 & 0.0004 & 0. & & 0.985 & 0.001 \\
\hline 1890 & 9852 & 0004 & .9863 & 0.0004 & & & & 0.0003 \\
\hline 891 & 3688 & & 0.8688 & .0011 & & & 9816 & \\
\hline 1891 & 0.9789 & 0.0012 & 0.9841 & 0.0012 & 0.9788 & 0.0008 & .9788 & 0.0008 \\
\hline 3000 & 1.000 & 0.001 & 1.000 & 0.001 & 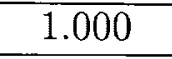 & & & .0002 \\
\hline 3000 & 1.000 & 1 & 1.000 & 1 & & & & .0001 \\
\hline 3001 & 0.9744 & & 0.9744 & .0008 & & & 0.984 & 0.0005 \\
\hline 3001 & 1.000 & 0009 & 1.000 & 0.0009 & 1.000 & 01 & 1.000 & .0001 \\
\hline 3002 & 1.000 & & 1.000 & .0006 & 0.9998 & & & \\
\hline 3002 & 1.000 & & 1.000 & & 1.000 & & 1.000 & .0001 \\
\hline 3003 & 955 & & 0.955 & 0.0035 & 0.9676 & & .9676 & 1.0009 \\
\hline 3003 & .9885 & 2 & 0.9942 & 12 & 0 . & 03 & 83 & \\
\hline 3004 & 9456 & & 56 & & & & .9754 & \\
\hline 3004 & 979 & & 0.979 & 0.0 & & & & .0003 \\
\hline 3005 & 883 & 3 & 83 & 13 & & & 9849 & .0003 \\
\hline 3005 & 923 & & 23 & 4 & & & & \\
\hline 3006 & 828 & & 9884 & & & & & \\
\hline 3006 & 000 & & 1.000 & 58 & & & & 0.0014 \\
\hline 3007 & 827 & & 000 & & & & 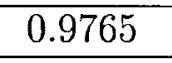 & 0.0005 \\
\hline 008 & 665 & & 6665 & & & & & 0.0003 \\
\hline 3009 & 71 & & 54 & & & & & 0.0003 \\
\hline 18 & 886 & & & & & & 31 & 003 \\
\hline 019 & 381 & & & & & & 797 & 003 \\
\hline 020 & & & & & & & & 03 \\
\hline 021 & & & & & & & 27 & \\
\hline 022 & 671 & & 71 & & & & 83 & \\
\hline 3023 & & & & & & & & 0.0003 \\
\hline 024 & 829 & & 29 & & & & 0.9777 & 0.0003 \\
\hline 025 & 541 & & 41 & & 74 & & 0.976 & 0.0004 \\
\hline 526 & & & & & & & & 0.0015 \\
\hline 27 & & & & & & & 0.9781 & 0.0017 \\
\hline 3029 & 9643 & & 9643 & & & & 9.9792 & 0.0003 \\
\hline 3030 & 942 & & 42 & & & 06 & 0.9782 & 0.0006 \\
\hline & & & & & & & 1.000 & 0.001 \\
\hline 3042 & & & & & & & 1.000 & 0.0017 \\
\hline 3044 & & & & & & & 1.000 & 0.0029 \\
\hline & 767 & & 9824 & & 18 & & 0.9822 & 0.0003 \\
\hline & & & & & & & & .0003 \\
\hline 102. & 9931 & 0.0014 & 0.0801 & 0.0014 & 0.9829 & 0.0003 & 0.9829 & 0.0 \\
\hline
\end{tabular}




\begin{tabular}{|c|c|c|c|c|c|c|c|c|}
\hline Run & $S 1_{E}$ & $\sigma$ & $S 2_{E}$ & $\sigma$ & $S 1_{E_{N}}$ & $\sigma$ & $S 2_{E_{N}}$ & \\
\hline 3048 & 0.9791 & 0.0014 & 0.9791 & 0.001 & & 0000 & & 9000 \\
\hline 3049 & .9673 & & 978 & & 0 & 04 & 0 & $\overline{004}$ \\
\hline 3050 & .9928 & & 0.9928 & 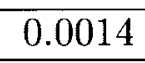 & 0.9802 & & & 00 \\
\hline 3051 & 852 & & 985 & 14 & 0.9812 & 0.0003 & 16 & .0003 \\
\hline 3052 & 9787 & & 97 & & 754 & $.00 \overline{3}$ & & \\
\hline 3054 & 9886 & 2 & 0.9886 & .0012 & 0.9817 & 0.0003 & 0.9855 & .0003 \\
\hline 3055 & 9345 & 13 & .9727 & 14 & 0.9797 & & 797 & 0.0003 \\
\hline 3056 & 713 & & 0.9311 & 14 & 0.9914 & & & \\
\hline 3057 & 12 & & 12 & & $0 . \overline{9335}$ & 0.0005 & .9652 & 0.0005 \\
\hline 3058 & 0.9721 & & 9721 & & 0.9822 & 03 & & 0.0003 \\
\hline 3059 & 413 & & 13 & & 0.9839 & 05 & & \\
\hline 3060 & 855 & & 27 & & .9779 & & 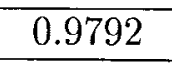 & \\
\hline 3061 & 853 & & 53 & & 0.9773 & & 69 & 00 \\
\hline 3062 & 719 & & 19 & 4 & 0.9788 & 03 & & \\
\hline 3068 & 00 & & & & 1.000 & & & \\
\hline 3069 & 925 & & 9925 & & 0.9 & & & 04 \\
\hline 3070 & 786 & & 86 & 4 & & & & \\
\hline 3071 & 712 & & 12 & & .9788 & & 00 & \\
\hline 3072 & 85 & & 85 & & 0.982 & & & \\
\hline 3073 & 778 & & 9778 & & 71 & & & 0.0004 \\
\hline 3074 & 00 & & 000 & & 721 & & & \\
\hline 3075 & 556 & & 56 & & & & & \\
\hline 3076 & 00 & & 24 & & & & 09 & \\
\hline 077 & & & & & & & 2 & \\
\hline 078 & $\overline{922}$ & & 22 & & & & 742 & \\
\hline 3079 & & & & & & & & \\
\hline 80 & & & & & & & & \\
\hline 3081 & 000 & & 00 & & 65 & & 738 & \\
\hline 3082 & 849 & & & & & & & \\
\hline 83 & 36 & & & & & & .9180 & \\
\hline 3084 & 478 & & 78 & & 588 & & 9688 & \\
\hline 085 & 797 & & & & & & & 0.0006 \\
\hline 86 & 694 & & 94 & & & & .9754 & 0.0004 \\
\hline 3087 & & & & & & & 0.9745 & 0.0004 \\
\hline 88 & & & & & & & 27 & 004 \\
\hline & 572 & & & & 0.9767 & & 0.9767 & 0.0004 \\
\hline & & & & & & & & \\
\hline 3125 & 1.000 & & & & 88 & & 38 & 0.0018 \\
\hline & 889 & & & & 15 & & 327 & 0.0004 \\
\hline & & & & & & & & 0.0005 \\
\hline & & 0.0012 & 0.0007 & 0.0042 & 0.9818 & 0.001 & 0.9854 & 0.001 \\
\hline
\end{tabular}




\begin{tabular}{|c|c|c|c|c|c|c|c|c|}
\hline Run & $S 1_{E}$ & $\sigma$ & $S 2_{E}$ & $\sigma$ & $S 1_{E_{\text {NoGas }}}$ & $\sigma$ & $S 2_{E_{\text {NoGas }}}$ & $\sigma$ \\
\hline 3129 & 1.000 & 0.0021 & 1.000 & 0.0021 & 0.9837 & 0.0005 & 0.9847 & 0.0005 \\
\hline 3130 & 0.9886 & 0.0018 & 0.9886 & 0.0018 & 0.984 & 0.0004 & 0.9834 & 0.0004 \\
\hline 3131 & 1.000 & 0.0052 & 1.000 & 0.0052 & 0.9782 & 0.0012 & 0.9836 & 0.0012 \\
\hline 3133 & 0.9562 & 0.0017 & 0.9562 & 0.0017 & 0.9811 & 0.0004 & 0.9805 & 0.0004 \\
\hline 3134 & 0.9837 & 0.0021 & 0.9679 & 0.0021 & 0.9827 & 0.0005 & 0.9827 & 0.0005 \\
\hline 3135 & 1.000 & 0.0017 & 1.000 & 0.0017 & 0.9792 & 0.0004 & 0.9792 & 0.0004 \\
\hline 3136 & 0.9826 & 0.0013 & 0.9941 & 0.0013 & 0.9815 & 0.0004 & 0.9815 & 0.0004 \\
\hline 3137 & 0.9882 & 0.0018 & 0.9766 & 0.0018 & 0.9774 & 0.0004 & 0.9768 & 0.0004 \\
\hline 3138 & 0.958 & 0.0017 & 0.958 & 0.0017 & 0.974 & 0.0003 & 0.9925 & 0.0003 \\
\hline 3139 & 1.000 & 0.0017 & 1.000 & 0.0017 & 0.9863 & 0.0004 & 0.9558 & 0.0004 \\
\hline 3140 & 0.9544 & 0.0018 & 0.9544 & 0.0018 & 0.9807 & 0.0004 & 0.982 & 0.0004 \\
\hline 3140 & 1.000 & 0.06324 & 1.000 & 0.06324 & 1.000 & 0.0365 & 1.000 & 0.0365 \\
\hline
\end{tabular}

Table 20: Table with the Scintillator efficiencies; comparison all cuts and no gas Cerenkov cut.

continued Scintillator efficiencies

The quantity $S 1_{E}$ represents the efficiency for the first scintillator in the electron arm with the gas cut applied, $S 2_{E}$ is the efficiency for the second scintillator detector in the electron arm with the gas cut applied, $S 1_{E_{\text {NoGas }}}$ is the efficiency for the first scintillator in the electron arm without the gas cut applied, and $S 2_{E_{\text {NoGas }}}$ is the efficiency for the second scintillator in the electron arm without the gas cut applied. 
VITA

\section{MARIUS COMAN}

2001-2005 Doctoral candidate in Physics, Florida International University.

1998-2000 M. Sc. (Physics), Florida International University.

1989-1994B. Sc. (Physics Engineer), Bucharest University, Romania.

2000- Member of American Physical Society (APS).

1998- Member of Thomas Jefferson National Accelerator Facility User Group.

1998- Research on strangeness electroproduction off hydrogen, multidimensional data analysis, participating in neutron form factor experiments, Thomas Jefferson National Accelerator Facility, Newport News, Virginia.

1994-1998 Physics Engineer, Contributions to International Atomic Energy Agency projects: Marine Environmental Assessment of the Black Sea Region - RER/2/003, Upgrading of the Environmental Radioactivity Surveillance Laboratory - TCP ROM/9/012, radioactivity monitoring of Cernavoda Nuclear Power Plant, radioactivity level survey of Kozlodui Nuclear Power Plant, Environmental Engineering Institute, Bucharest Romania.

\section{PUBLICATIONS AND PRESENTATIONS}

1. Determination of radio nuclides distribution in soil and dose assessment, M.Coman et. al., research contract with Romanian Research Ministry.

2. Identification and determination of influencing parameters which intercess in radioactivity levels assessment, M.Coman et. al., research contract with Romanian Research Ministry. 
3. Gamma in situ spectrometry calibration methods and analysis, Environmental Engineering Institute, M.Coman et. al., research contract with Romanian Research Ministry.

4. Elaboration of a program for radioactivity levels survey of Kozlodui nuclear power plant, M.Coman et. al., research contract with Romanian Research Ministry.

5. The Hall A Diffusion Aerogel Counter, presented at the American Physical Society meeting, Division of Nuclear Physics, October 1999, Pacific Grove, CA.

6. E98-108 Exclusive Kaon Electroproduction off Hydrogen, presented at the American Physical Society meeting (APS), April 08 2003, Philadelphia, PA.

7. The Hall A Aerogel Cerenkov Detector - M.S. thesis, FIU, 2000.

8. Monte Carlo simulations on Rosenbluth separation in kaons electroproduction reaction, $\mathrm{H}\left(\mathrm{e}, \mathrm{e}^{\prime} \mathrm{K}\right)$, presented at the APS, Division of Nuclear Physics, October 2000, Williammsburg, VA.

9. Unveilling the non manifest reality - FIU, Society of Physics Students, FIU, 2002. 10. Cross sections and Rosenbluth separations from kaon electroproduction on protons, experiment E98-108, Jefferson Lab, Newport News, VA, December 2004. 